\title{
Electron Scattering
}

\author{
Ryan Alan Snyder \\ Washington, DC \\ B.A., Kenyon College, 1999 \\ M.A., University of Virginia, 2004
}

A Dissertation presented to the Graduate Faculty of the

University of Virginia in Candidacy for the Degree of

Doctor of Philosophy

Department of Physics

University of Virginia

May, 2010

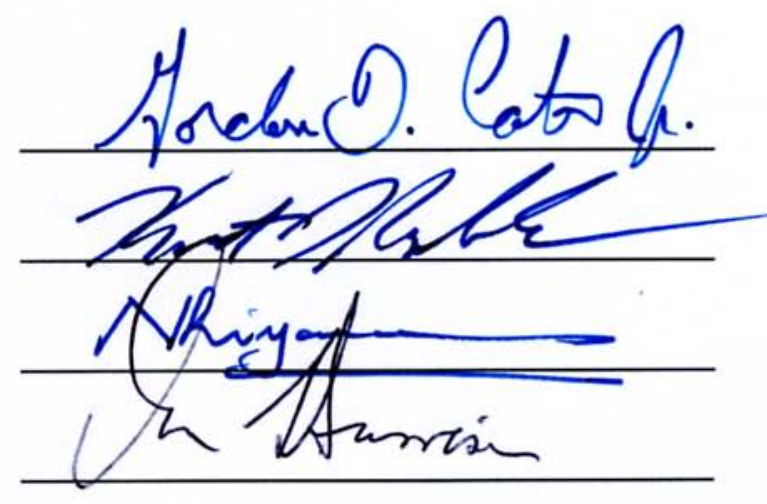


(c) Copyright by Ryan Alan Snyder

All Rights Reserved

May 2010 


\begin{abstract}
This thesis reports on the 2005 HAPPEX parity-violating measurements $A_{P V}$ in elastic scattering of $3 \mathrm{GeV}$ electrons off hydrogen and ${ }^{4} \mathrm{He}$ targets at $\left\langle\theta_{\text {lab }}\right\rangle \approx 6.0^{\circ}$ carried out in Hall A at the Thomas Jefferson National Accelerator Facility in Newport News, VA. The ${ }^{4}$ He result is $A_{P V}^{\mathrm{He}}=+6.40 \pm 0.23$ (stat) \pm 0.12 (syst) ppm. The hydrogen result is $A_{P V}^{\mathrm{H}}=-1.60 \pm 0.12$ (stat) \pm 0.05 (syst) ppm. These results significantly improve the constraints on the electric and magnetic strange form factors $G_{E}^{s}$ and $G_{M}^{s}$. We extracted $G_{E}^{s}=-0.02 \pm 0.014$ (stat) \pm 0.007 (syst) at $\left\langle Q^{2}\right\rangle=0.077 \mathrm{GeV}^{2}$ from the ${ }^{4}$ He result and the linear combination $G_{E}^{s}+0.088 G_{M}^{s}=0.007 \pm 0.011$ (stat) \pm 0.004 (syst) \pm 0.005 (FF) at $\left\langle Q^{2}\right\rangle=0.109 \mathrm{GeV}^{2}$ from hydrogen. These results provide new limits on the role of strange quarks in the nucleon charge and magnetization distributions.

This thesis reports on both the ${ }^{4} \mathrm{He}$ and hydrogen analysis of the 2005 data set with a particular focus on the background contamination for each measurement. An additional focus here is given to understanding and minimizing helicity-correlated asymmetries on the electron beam, systematic errors we needed to control at levels well below that of the measured parity-violating asymmetries $A_{P V}$.
\end{abstract}




\section{Acknowledgements}

First, thanks must go to my advisor Gordon Cates who provided me with the freedom an aspiring scientist wants with the important guidance one will also inevitably need. In partic-

ular, I would like to thank Gordon for his understanding this past year as some struggles in my personal life have prolonged the writing of this thesis. An important thank you must also be extended to Kent Paschke who even though burdened by his enormous responsibilities with HAPPEX, always found time to answer my questions with patience, enthusiasm, and humor. He is an enormous asset to the field of physics, and future students will be fortunate to come across him in their training.

I would also like to thank Krishna Kumar and Paul Souder for not only doing everything in their power to make the experiment run the way it should, but for representing to me exemplary scientists in their constant searching for information, attention to detail, and passion for what they do. Theirs is an example I will always remember fondly.

The three other HAPPEX PhD students Bryan Moffit, Lisa Kaufman, and Hachemi Benaoum also need to be thanked for the enormous energy they put into this effort and for their good natured cooperation throughout many tiring and trying times. Additonally, members of the HAPPEX collaboration who, regrettably, deserve more attention than only 
a brief thank you here include Bob Michaels, David Armstrong, David Lhuillier, and Rich Holmes.

This work certainly could not have been completed without the cooperation of Jefferson Lab's polarized source group. Matt Poelker, John Hansknecht, and Joe Grames helped with our numerous requests for tuning our setup or obtaining additional equipment, and were all particularly helpful and understanding during long days in the injector tunnel as we were setting up the source for the experiment run. Their help and understanding were incalculable and they deserve to know how much it was appreciated. I'd also like to acknowledge Brain Humensky who was patient in explaining to me various aspects of the polarized source as I began learning and who was always willing even after starting a new job to discuss any questions or problems Kent, Lisa, or I had as we were attempting to make our source setup work the way we hoped.

Scott Rohrbaugh and Jaideep Singh I will miss as they were as wonderful a pair one could ask to have in one's graduate research group. Both had passionate interests outside of physics, were great listeners, and were simply fun to be around. Jaideep in particular spent many hours listening to my frustrations and opinions about politics and the wider world and he did so with a generosity that I appreciated more than he can know or I can express. Al Tobias also deserves my thanks for his time and patience in introducing me to much around the target lab as I was just beginning research with the group.

Many thanks must be extended to my good friends from college Brendan, Mike, and Mick, who have over the years provided me with the kind of support that only true friends can. Brendan, in particular, must be singled out as he provided me with as much support 
and understanding as is humanly possible as I completed the writing of this thesis from his apartment.

Lastly, to my parents and brother Cary, whose unceasing love, encouragement and understanding are forever a reminder that in the cosmic crapshoot of life, no one lucked out more than I did. 
To my grandfather, James Christian Ritchey (1926-2009)

Seldom does God bless this world with a better man. I won't come across your like often. With prayerful thoughts for your safe passage. 
Upon the subject of education, not presuming to dictate any plan or system respecting it, I can only say that I view it as the most important subject which we as a people can be engaged in.

—Abraham Lincoln, March 8, 1832 


\section{Contents}

Abstract $\quad$ iv

Acknowledgements $\quad$ v

List of Figures $\quad$ xi

List of Tables $\quad$ xiv

1 Introduction 1

1.1 Investigating Strange Quarks in the Nucleon . . . . . . . . . . . . . . . . . 3

1.1.1 Calculating the Strange Form Factors . . . . . . . . . . . . . . 5

1.2 Helicity-Correlated Electron Beam Asymmetries . . . . . . . . . . . . . . 7

2 Theory and Formalism 9

2.1 Electron-Nucleon Scattering . . . . . . . . . . . . . . . . . . . . . . . 9

2.1.1 Electromagnetic Scattering . . . . . . . . . . . . . . . . 10

2.1.2 Electron-Nucleus Scattering . . . . . . . . . . . . . . . . . . 13

2.1.3 Neutral Weak Form Factors . . . . . . . . . . . . . . . . . . . . . 14

2.1.4 Relationship Between Electromagnetic and Weak Form Factors . . . . 15

2.2 Parity Violation . . . . . . . . . . . . . . . . . . . . . . . . 19

2.3 Spinless Isoscalar Nuclei . . . . . . . . . . . . . . . . . . . . . 22

3 Experimental Design 23

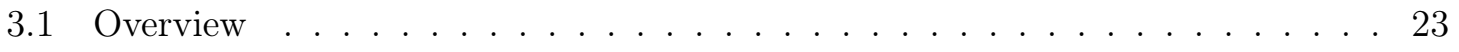

3.2 Choice of Kinematics . . . . . . . . . . . . . . . . . . . . . . 25

3.3 Experimental Technique . . . . . . . . . . . . . . . . . 27

3.4 Fast Helicity Reversal . . . . . . . . . . . . . . . . . . . . . . . . . 28

3.5 Integration . . . . . . . . . . . . . . . . . . . . 29

3.6 Statistical Error . . . . . . . . . . . . . . . . . . . . 30

3.7 Systematic Error . . . . . . . . . . . . . . . . . . . . 34

3.8 Beam Property Asymmetries . . . . . . . . . . . . . . . . 35

3.9 Slow Helicity Reversal . . . . . . . . . . . . . . . . . . . . . . . . 37

3.10 Backgrounds . . . . . . . . . . . . . . . . . . . 37

3.11 Polarimetry . . . . . . . . . . . . . . . . . . . . 38 
4 Experimental Apparatus 40

4.1 Accelerator at TJNAF . . . . . . . . . . . . . . . . . 40

4.2 Polarized Source . . . . . . . . . . . . . . . . . . . . 41

4.2.1 GaAs Photocathode . . . . . . . . . . . . . . . . . . 42

4.2 .2 Source Optics . . . . . . . . . . . . . . . . . . . . . . 45

4.3 Beamline . . . . . . . . . . . . . . . . . . . . . . 49

4.3.1 Beam Energy Measurement . . . . . . . . . . . . . . . . . 49

4.3 .2 Current Monitors . . . . . . . . . . . . . . . . . . 53

4.3.3 Beam Position Monitors . . . . . . . . . . . . . . . . . 55

4.3.4 Modulation Coils . . . . . . . . . . . . . . . . . . . 56

4.4 Target and Raster . . . . . . . . . . . . . . . . . . 57

4.5 Hall A Spectrometers . . . . . . . . . . . . . . . . . . . . 60

4.5.1 High Resolution Spectrometers . . . . . . . . . . . . . . . . . 60

4.5 .2 Septum Magnets . . . . . . . . . . . . . . . . . . . . . 6 . . . . . . . 62

4.5.3 Hall A Standard Detector Package . . . . . . . . . . . . . . . 63

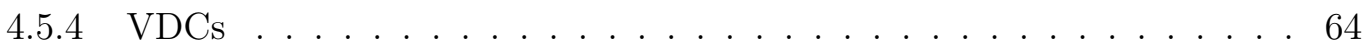

4.5.5 HAPPEX Focal-Plane Detectors . . . . . . . . . . . . . 67

4.6 Data Acquisition . . . . . . . . . . . . . . . . . . . . 70

4.7 Polarimetry . . . . . . . . . . . . . . . . . . . . . . 72

4.7.1 Møller Polarimeter . . . . . . . . . . . . . . . . . . . 72

4.7 .2 Compton Polarimeter . . . . . . . . . . . . . . . 74

$4.8 Q^{2}$ Profile Scanner . . . . . . . . . . . . . . . . . 75

$\begin{array}{lll}5 & \text { Polarized Electron Source } & \mathbf{7 7}\end{array}$

5.1 Circular Polarization and Setup of the Pockels Cell . . . . . . . . . . . 78

5.1 .1 Circular Polarization . . . . . . . . . . . . . . . . . . . 78

5.1 .2 Alignment of the Pockels Cell . . . . . . . . . . . . . . . . . 80

5.2 Sources of Helicity-Correlated Beam Asymmetries . . . . . . . . . . . . . . 82

5.2.1 Polarization Induced Transport Asymmetry (PITA) . . . . . . . . . . . 82

5.2 .2 PITA Slopes . . . . . . . . . . . . . . . . . . . . 85

5.2 .3 Spatial Variation of $\Delta \ldots \ldots \ldots \ldots 6$

5.2 .4 Laser Skew Effect . . . . . . . . . . . . . . . . . . . . . . . . 88

5.2 .5 Steering . . . . . . . . . . . . . . . . . . . 97

5.2.6 Charge Asymmetry Structure . . . . . . . . . . . . . . . . . . . . 98

5.2.7 Vacuum Window and Rotatable Half-Wave Plate . . . . . . . . . . . . 100

5.2 .8 QE Anisotropy Gradients . . . . . . . . . . . . . . . . . . . . . 103

5.2 .9 Cross-talk (Electronic and otherwise) . . . . . . . . . . . . . . 103

5.3 Minimization of beam $A_{\mathrm{LR}}$ 's . . . . . . . . . . . . . . . . . . . . . . 104

5.3.1 Pockels cell selection . . . . . . . . . . . . . . . . . . . . . . 104

5.3.2 Minimizing the Laser Skew Effect . . . . . . . . . . . . . . . . . . 107

5.4 Controlling the PITA Effect . . . . . . . . . . . . . . . . . . . 109

5.4 Helicity Slow Reversal . . . . . . . . . . . . . . . . . . . . . . . 114

5.4 .2 Feedback . . . . . . . . . . . . . . . . . . . . . 115

5.4 .3 Adiabatic Damping . . . . . . . . . . . . . . . . . . 116

$5.52005{ }^{4} \mathrm{He}$ and H Source Setups . . . . . . . . . . . . . . . . 117 
$5.5 .12005{ }^{4} \mathrm{He}$ Tunnel Setup . . . . . . . . . . . . . . . . . . . . . . . . . . . . . . . . . . . . .

5.5 .22005 H Tunnel Setup . . . . . . . . . . . . . . . . . . 121

6 Analysis $\quad 128$

6.1 Asymmetry Analysis . . . . . . . . . . . . . . . . . . . . . . 128

6.1 .1 Raw Asymmetry . . . . . . . . . . . . . . . . . . . . . . . . . 128

6.1.2 Beam Modulation Analysis . . . . . . . . . . . . . . . . . 130

6.1 .3 Linearity . . . . . . . . . . . . . . . . . . . . . . . . . . . . . 138

6.1.4 Transverse Beam Asymmetry . . . . . . . . . . . . . . . . . . . . 139

$6.2 Q^{2}$ Determination . . . . . . . . . . . . . . . . . . . . . . . . . . . . . . . . . . .

6.3 Beam Polarization . . . . . . . . . . . . . . . . . . . . 147

6.4 Backgrounds . . . . . . . . . . . . . . . . . . . . . . . . . . . . . 148

$6.5{ }^{4}$ He Quasielastic Scattering . . . . . . . . . . . . . . . . . . . . . . 149

6.5.1 Aluminum Target Windows . . . . . . . . . . . . . . . . . . . . 160

6.5.2 Rescattering in the Spectrometer . . . . . . . . . . . . . . . . . 160

6.5.3 Poletip . . . . . . . . . . . . . . . . . . . . 163

6.6 Effective Kinematics ． . . . . . . . . . . . . . . . . . . . . . . . . . . 168

6.7 Physics Asymmetry . . . . . . . . . . . . . . . . . 168

7 Results and Conclusions $\quad 172$

7.1 Results . . . . . . . . . . . . . . . . . . . . . 172

7.2 Interpretation and Conclusions . . . . . . . . . . . . . . . . 179

7.3 Future Measurements . . . . . . . . . . . . . . . . . . . . . . . 181

7.3.1 Strange Vector Form Factors . . . . . . . . . . . . . . . . . . . . 182

$7.3 .2 \quad$ Nuclear Structure . . . . . . . . . . . . . . . . . . . . . . . . . 182

7.3.3 Physics Beyond the Standard Model . . . . . . . . . . . . . . . . 183

$\begin{array}{ll}\text { Bibliography } & 185\end{array}$ 


\section{List of Figures}

1.1 Views of the proton's valence quarks and gluons and $q \bar{q}$ pairs in the sea $\ldots .5$

2.1 Feynman diagrams for electron-nucleon scattering. . . . . . . . . . . . . . 10

3.1 Overview of the experimental apparatus . . . . . . . . . . . . . . . 24

3.2 Figure of Merit for hydrogen measurement . . . . . . . . . . . . . . . . . 25

3.3 Relative Figure of Merit for helium measurement . . . . . . . . . . . . . . 27

3.4 A pseudorandom helicity sequence . . . . . . . . . . . . . . . 29

4.1 Schematic of TJNAF accelerator and the experimental halls . . . . . . . . . . 42

4.2 Structure of bulk and strained GaAs photocathodes . . . . . . . . . . . . . 43

4.3 Structure of the strained-layer and superlattice cathode . . . . . . . . . . . 44

4.4 The JLab polarized source laser system . . . . . . . . . . . . . . . . . . 46

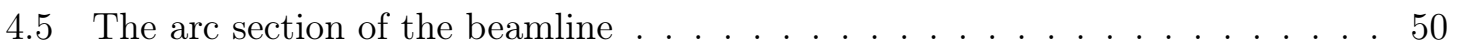

4.6 Layout of the eP device . . . . . . . . . . . . . . . . . 51

4.7 Schematic of the beam current monitors . . . . . . . . . . . . . . 54

4.8 Schematic of a stripline beam position monitor . . . . . . . . . . . 56

4.9 Picture of the racetrack target cell . . . . . . . . . . . . . . . 57

4.10 Schematic of the High Resolution Spectrometer . . . . . . . . . . . . . . 61

4.11 A simulated elastic peak focused onto the the focal plane by the HRS . . . . . 63

4.12 A view looking down of the HRS setup with the septum magnet installed . . 64

4.13 Sieve-slit hole pattern used for optimization of spectrometer transfer matrices 65

4.14 Diagram of the VDC planes . . . . . . . . . . . . . . . . 6 . . . . . . . 65

4.15 Configuration of wire chambers . . . . . . . . . . . . . . 67

4.16 Focal plane detector showing scattered electrons entering from the side . . . 68

4.17 A cutaway view of the HAPPEX detector quartz and brass layers . . . . . . . . . 69

4.18 Circuit diagram of one channel of an integrating ADC . . . . . . . . . . . . . 71

4.19 Layout of the Hall A Møller polarimeter . . . . . . . . . . . . . . . . 73

4.20 Layout of the Compton Polarimeter. The top diagram displays the polarimeter from the side while the bottom shows a view from above the compton chicane. . . . . . . 75

4.21 A schematic of the profile scanner . . . . . . . . . . . . . 76

5.1 Polarization ellipses with symmetric and antisymmetric phase shifts . . . . . . 84

5.2 A plot of $A_{Q}$ vs. offset voltage needed to measure PITA slope . . . . . . . 86

5.3 Helicity-correlated shifts in electron beam's intensity profile . . . . . . . . . . 87

5.4 Diagram of ray incident on the Pockels cell at some skew angle . . . . . . . . 91 
5.5 Diagram of a light ray incident on the Pockels cell at an angle $\rho . \ldots . . . .93$

5.6 Diagram of light ray projection needed to model skew effect . . . . . . . . . 95

5.7 Charge asymmetry structure for toggle, quad-random, and pair-random modes 99

5.8 Charge asymmetry vs. elasped time over a helicity window . . . . . . . . . . 100

5.9 Setup used to measure birefringence gradients . . . . . . . . . . . . . . 105

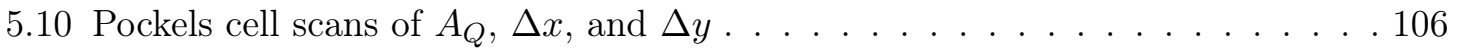

5.11 Pockels cell scans of $A_{Q}, \Delta x$, and $\Delta y$ displaying skew offset . . . . . . . . 108

5.12 A measure of the source's sensitivity to the yaw adjustment . . . . . . . . . 110

5.13 Source's sensitivity to yaw after adjustments . . . . . . . . . . . . . 111

5.14 Schematic of optics used to produce circularly polarized light . . . . . . . . 112

5.15 Analyzing power orientations for maximizing and minimizing $A_{Q} \ldots \ldots$

$5.16 \Delta x$ and $\Delta y$ at BPM's $4 \mathrm{~A}$ and $4 \mathrm{~B}$ for $2005{ }^{4} \mathrm{He}$ run . . . . . . . . . . 120

$5.17 \Delta x(\mu \mathrm{m})$ vs. RHWP angle with IHWP OUT . . . . . . . . . . . . . . . . . . . . . . . . .

$5.18 \Delta x(\mu \mathrm{m})$ vs. RHWP angle with the IHWP IN . . . . . . . . . . . . . . 124

$5.19 A_{Q}(\mathrm{ppm})$ vs. RHWP angle with IHWP IN . . . . . . . . . . . . 125

$5.20 A_{Q}(\mathrm{ppm})$ vs. RHWP angle with the IHWP OUT $\ldots \ldots \ldots \ldots \ldots$

$5.21 \Delta x$ at injector BPM's for IHWP OUT . . . . . . . . . . . . . 125

$5.22 \Delta y$ at injector BPM's for IHWP IN . . . . . . . . . . . . . . 126

$5.23 \Delta x$ at injector BPM's for IHWP IN . . . . . . . . . . . . . . . . . . . . . . . . . . . . . . . . . .

$5.24 \Delta y$ at injector BPM's for IHWP OUT . . . . . . . . . . . . . . . . . . . . . . . . . . . . . . . . .

$5.25 \Delta x$ and $\Delta y$ at BPM's $4 \mathrm{~A}$ and $4 \mathrm{~B}$ for $2005 \mathrm{H}$ run $\ldots \ldots \ldots . \ldots . \ldots . \ldots$

$6.1 A_{\text {raw }}$ pulse-pair distributions on a log scale for $2005 \mathrm{H}$ run . . . . . . . . . 130

6.2 A beam modulation cycle displaying detector response vs. event number . . . 133

$6.3 A_{\text {corr }}$ for 2005 He data arranged by IHWP state . . . . . . . . . . . . . . . . 134

$6.4 A_{\text {corr }}$ for HAPPEX-H 2005 data arranged by $\lambda / 2$ plate state . . . . . . . . . 135

$6.5{ }^{1} \mathrm{H}$ uncorrected and ${ }^{4} \mathrm{He}$ corrected asymmetries for all the final 2005 HAPPEX data . . . . . . . . . . . . . . . . . . . . . . . . . . . . . . . . . . . . .

6.6 Sample fit of reconstructed electron energy from central sieve hole . . . . . . . 144

6.7 Typical 2005 Hydrogen $Q^{2}$ run . . . . . . . . . . . . . . . . . . . 146

6.8 A typical $Q^{2}$ scanner run . . . . . . . . . . . . . . . . . . 147

6.9 Analyzed Compton Polarimeter data for 2005 HAPPEX-He run . . . . . . . . 148

6.10 Analyzed Compton Polarimeter data for 2005 HAPPEX-H run . . . . . . . . 149

6.11 The difference in measured $p$ from central spectrometer $p_{o} \ldots$. . . . . . . . 150

6.12 Transverse vs. Dispersive HAPPEX detector data collected from a $24 \mathrm{~cm}$ Carbon target . . . . . . . . . . . . . . . . . . 151

$6.13 \mathrm{~A}^{4} \mathrm{He}$ momentum distribution in rotated coordinates . . . . . . . . . . . 153

6.14 Deviation from central momentum $d p$ vs. dispersive coordinate $\mathrm{x}(\mathrm{m})$ in rotated coordinates . . . . . . . . . . . . . . . . 154

$6.15 E_{\text {diff }}$ spectrum for ${ }^{4} \mathrm{He}$ "counting mode" spectrometer run 2271 . . . . . . . 156

6.16 The spectrum of the in-plane scattering angle for the left and right arms. . . . 157

6.17 Target $\phi$ vs. focal-plane y . . . . . . . . . . . . . . . 157

6.18 Focal plane $x$ vs. $y$ in rotated coordinates . . . . . . . . . . . . . . 158

6.19 A log plot of the x-rotated coordinate at the left HAPPEX detector . . . . . . 159

6.20 A log plot of the x-rotated coordinate at the right HAPPEX detector . . . . . 159 
$6.21 E_{\mathrm{dep}} / E_{\mathrm{o}}$ for Helium at different dipole momentum settings . . . . . . . . . . . 164

$6.22{ }^{4} \mathrm{He}$ inelastic spectrum plotted versus deviation from the elastic peak in $\mathrm{MeV} 165$

$6.23 E_{\text {dep }} / E_{\mathrm{o}}$ for Hydrogen at different dipole momentum settings . . . . . . . . 166

6.24 Hydrogen inelastic spectrum plotted vs. deviation from the elastic peak in MeV167

7.1 $A_{L R}$ measurements from $Q^{2}=0.09-0.11 \mathrm{GeV}^{2}$, including the 2005 results .177

7.2 $A_{L R}$ measurements from $Q^{2}=0.077-0.11 \mathrm{GeV}^{2}$, including the 2004 results .178

7.3 HAPPEX 2004 and 2005 results . . . . . . . . . . . . . . . . 180

7.4 World data (except for HAPPEX-III) of $G_{E}^{s}+\eta G_{M}^{s}$ at forward angle on proton targets as a function of $Q^{2} \ldots \ldots . \ldots 182$ 


\section{List of Tables}

2.1 Electromagnetic and weak charges for the electron and light quarks. . . . . 15

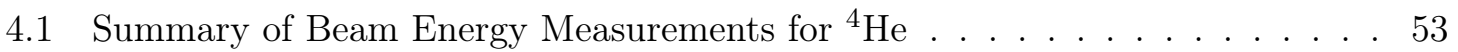

4.2 Dimensions of target used in the experiment . . . . . . . . . . . . . 58

4.3 Parameters for He target operation . . . . . . . . . . . . . . . . . 59

4.4 Hall A HRS general characteristics . . . . . . . . . . . . . . . . . 62

5.1 Average beam asymmetries for the 2005 HAPPEX-II runs . . . . . . . . 118

6.1 Average beam modulation slopes for each BPM during $2005 \mathrm{H}$ run . . . . . . 132

6.2 Average $\mathrm{x}$ and $\mathrm{y}$ beam asymmetries for both 2005 HAPPEX-II runs . . . . . . 136

6.3 Raw and corrected asymmetries (in ppm) displayed by IHWP state . . . . . . 136

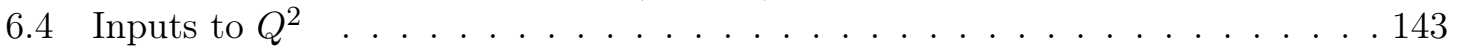

6.5 The fixed parameters used in the fit of the $E^{\prime}$ distributions . . . . . . . . . 144

6.6 Systematic Errors in $Q^{2} \ldots \ldots \ldots$. . . . . . . . . . . . . . 145

6.7 ADC weighted $Q^{2}$ for the 2005 runs of HAPPEX-H and -He . . . . . . . . 147

6.8 Summary of corrections and errors for all terms in ${ }^{4} \mathrm{He} A_{\text {phys }} \ldots \ldots$. . . . . 169

6.9 Summary of corrections and errors for all terms in ${ }^{1} \mathrm{H} A_{\text {phys }} \ldots \ldots$. . . . . 169

6.10 Contributions to ${ }^{4} \mathrm{He} A_{\text {phys }}$ systematic error . . . . . . . . . . . . . . 170

6.11 Contributions to ${ }^{1} \mathrm{H} A_{\text {phys }}$ systematic error . . . . . . . . . . . . . 171

7.1 Vector and axial electroweak radiative corrections . . . . . . . . . . . . 173

7.2 Electroweak Coupling Constants . . . . . . . . . . . . . . . . . . . . 174

7.3 Values of Effective Kinematics for $A_{\mathrm{NS}}$ calculation . . . . . . . . . . . . 175

7.4 Values of Form Factors needed for $A_{\mathrm{NS}}^{\mathrm{He}}$ and $A_{\mathrm{NS}}^{\mathrm{H}}$ calculations $\ldots \ldots . . . .175$

7.5 Calculated $A_{\mathrm{NS}}^{H}, A_{\mathrm{NS}}^{H e}$ and measured $A_{L R}^{H, H e}$ for 2005 run . . . . . . 176 


\section{Chapter 1}

\section{Introduction}

In 1918 Emmy Noether made the profound suggestion that for every symmetry in nature, there exists a corresponding conserved quantity [1]. For example, the invariance of physical laws under translations in space $x \rightarrow x+\Delta x$ and time $t \rightarrow t+\Delta t$ suggest, respectively, that momentum and energy are conserved, while invariance upon rotation $\phi \rightarrow \phi+\Delta \phi$ suggests the same for angular momentum. Of interest to our work is the parity quantum number whose conservation follows from the invariance of the electromagnetic and strong interactions under spatial inversion $(x, y) \rightarrow(-x,-y)$. After Lee and Yang reviewed the experimental evidence related to the weak interaction, they claimed such interactions did not obey this spatial inversion symmetry [2]. And in 1956, Wu, Ambler, and others confirmed these parity-violating claims in their famous polarized ${ }^{60} \mathrm{Co}$ beta-decay experiment [3], a discovery that led to detailed amendments in the theory of weak interactions such as the $V$ $A$ theory published by Feynman and Gell-Mann and independently by two other theorists, R. Marshak and E. Sudarshan [5]. 
Weak neutral currents, interactions mediated by the $Z^{o}$ boson with no electric charge, were first observed in 1973 when muon neutrinos and antineutrinos were scattered off electrons and nuclei to yield a cross section comparable in magnitude to other charged-current weak processes. However, these experiments were not able to determine to what extent, or whether, the weak neutral current was parity-violating. In 1978, the E122 experiment at the Stanford Linear Accelerator Center (SLAC) measured the parity-violating asymmetry in the polarization-dependedent cross section of the scattering of longitudinally polarized electrons off deuterium. The measured asymmetry of $\sim 10^{-4}$ indicated a parity-violating effect consistent with the first renormalizable theories of the weak interactions developed by Weinberg and Salam [4]. E122's measurement played an important role in the establishment of the Weinberg-Salam model as the standard model of electroweak interactions [5]. In order to search for parity-violation in electron scattering at the level of accuracy necessary to test the Weinberg-Salam model, the collaboration at SLAC assembled a unique high-intensity source of polarized electrons that were emitted from a surface-treated Galium Arsenide (GaAs) crystal exposed to circularly polarized laser light. An electro-optical device known as a Pockels cell was used to reverse the polarization of the laser light, and hence the helicity of the electron beam itself [5].

Many of techniques pioneered by E122 have since been used to probe the structure of the nucleon, including two experiments reported on here, HAPPEX II-Helium and HAPPEX II-Hydrogen. The basic idea of the experiments was to measure the elastic scattering of electrons from hydrogen and helium targets and compare the cross sections when electron spins were aligned parallel and antiparallel to their direction of motion. The parity-violating 
nature of the weak force accounts for the differences between the two cross sections, and as will be discussed, using parity-violation allows for differences in the two cross sections to be compared. When considering the common scale factors between them, only the asymmetry in integrated detector signals was needed to access the the strange quark contributions to the charge and magnetization distributions, the electric and magnetic strange form factors $G_{E}^{s}$ and $G_{M}^{s}$ respectively. Differences in the scattering cross sections from a helium target were sensitive to $G_{E}^{s}$ while those from hydrogen were sensitive to a linear combination of $G_{E}^{s}$ and $G_{M}^{s}$. The HAPPEX measurements presented critical experimental challenges due to the stringent requirements on the properties of the polarized electron beam, and these challenges had to be met in order to make a $\sim 10 \%$ measurement on the tiny asymmetries involving $\sim 1.6 \mathrm{ppm}$ in the case of hydrogen and $\sim 6.4 \mathrm{ppm}$ in the case of helium.

\subsection{Investigating Strange Quarks in the Nucleon}

The proton can be thought of a system containing three valence quarks (Figure 1.1a) bound by the strong force of Quantum Chromodynamics (QCD). These forces generate a collection of gluons and quark-antiquark $(q \bar{q})$ pairs that are commonly referred to as "sea quarks" (Figure 1.1b). An open question is the role of the sea in the fundamental properties of the nucleon. In 1988, the European Muon Collaboration (EMC)[6] published data on spindependent structure functions indicating that little of the proton spin is carried by the spins of the quarks; candidates for the missing spin included gluons and orbital angular momentum. The EMC data also suggested that significant spin is carried by the strange quarks. 
The EMC result inspired much theoretical activity directed towards better understanding this sea of gluons and $q \bar{q}$ pairs. A possibility suggested by Kaplan and Manohar was that strange quarks might contribute to the proton's charge radius and magnetic moment [7], and that accessing this contribution might be possible through measurement of the weak neutral-current amplitude in lepton-nucleon scattering.

An often discussed effect of strange quarks in the proton is the contribution to the proton's momentum revealed by charm production in deep inelastic neutrino scattering [8]. Their contribution to the mass of the proton comes from the matrix element $\langle P|s \bar{s}| P\rangle$, and a value of about $10 \%$ is inferred from the "sigma term"* in $\pi-N$ scattering [9]. Contributions to the spin of the proton may be inferred from the first moment $\Gamma_{1}$ of the spin structure functions $g_{1}(x)$ for the proton or the neutron, together with several other assumptions. Next-to-leading order QCD analyses have been done by many groups [10, 11, 12, 13], and the fraction of the nucleon's spin due to the spin of strange quarks, $\Delta s$, was found to be about -0.1 [14] in units of $\hbar / 2$. The error on $\Delta s$ in [14] is $\sim 0.05$, which arises from theoretical issues including $S U(3)_{f}$ symmetry breaking.

Using parity-violating electron scattering off of various nuclei allows access to another strange-quark-sensitive observable, the matrix element $\left\langle p\left|\bar{s} \gamma_{\mu} s\right| p\right\rangle$ where $s$ is the strange quark spinor. This quantity can be expressed in terms of the strange elastic form factors $G_{E}^{s}$ and $G_{M}^{s}$, which as the four momentum transfer, $Q^{2}$, approaches zero, can be related the proton's strangeness radius $\rho_{s}$ and strange magnetic moment $\mu_{s}$, respectively. Jefferson Lab in Newport News, VA, has proven to be an outstanding facility for doing such experiments, the first being the Hall A Proton Parity Experiment (HAPPEX) in 1998 and 1999, which

\footnotetext{
${ }^{*}$ The sigma term is defined as $\sigma_{p}=\frac{1}{4 M_{p}}\left(m_{u}+m_{d}\right)\langle P|\bar{u} u+\bar{d} d| P\rangle$.
} 


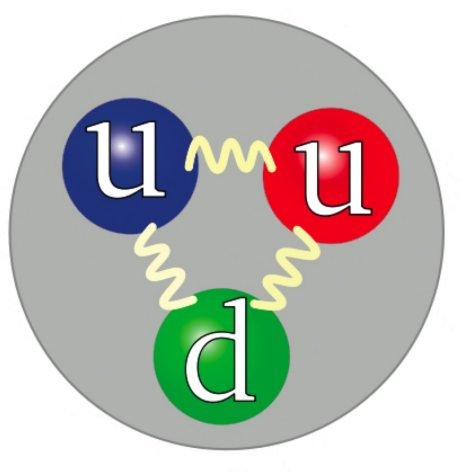

(a)

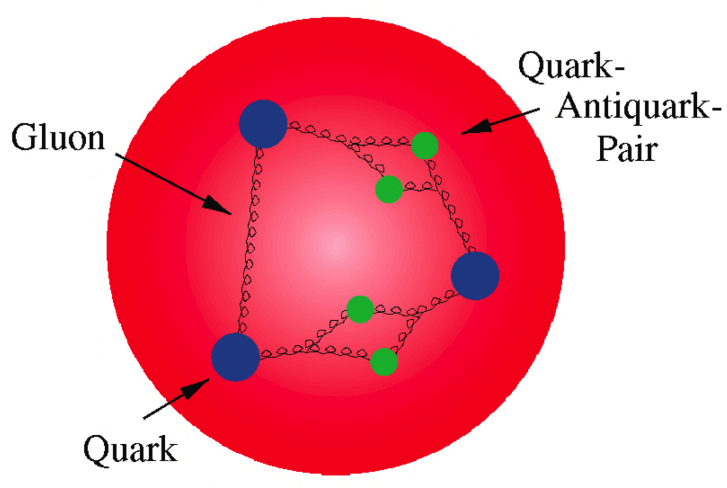

(b)

Figure 1.1: Views of the proton's (a) valence quarks and (b) the gluons and quark-antiquark pairs in the sea

measured the linear combination $G_{E}^{s}+0.39 G_{M}^{s}$ at $Q^{2}=0.477 \mathrm{GeV}^{2}$. This was followed by the experiments reported here, HAPPEX II-Helium and HAPPEX II-Hydrogen, which measured $G_{E}^{s}$ at $Q^{2}=0.077 \mathrm{GeV}^{2}$ and $G_{E}^{s}+0.09 G_{M}^{s}$ at $Q^{2}=0.109 \mathrm{GeV}^{2}$, respectively.

\subsubsection{Calculating the Strange Form Factors}

A number of different models have been used to estimate the magnitude of the strange form factors, and a theoretical review of the origins and limitations of many of them can be found in [15]; discussed here is a very broad qualitative picture along with some predictions.

Unfortunately, this estimation problem cannot be addressed by perturbative QCD as $m_{s} \sim \Lambda_{Q C D}$ making the energy scale too low for such calculations. Jaffe [16] used a vector meson dominance (VDM) model and dispersion analysis, and later more precise form factor data was used by Hammer et al. [97] to update this analysis. The "kaon loop" picture, where the origin of the spatial separation of $\bar{s}$ and $s$ comes from a $\Lambda-K$ component to the proton's wave function, was considered by Koepf, Henley and Pollock [18] within the context 
of the constituent quark model, and by Musolf and Burkardt [19] using hadron scattering data. This last result was close to the VMD approach, but Geiger and Isgur [20] showed that summing over a complete set of strange meson-baryon intermediate states results in cancellations that reduce $\mu_{s}$ to a very small value.

Efforts have been made to use Chiral perturbation theory $(\chi P T)$ to compute the leading $Q^{2}$ behavior of $G_{E}^{s}$ and $G_{M}^{s}$, but the models include large uncertainties [21]. Lattice QCD techniques will lead to the most rigorous estimate of the strange form factors, but the calculations remain very challenging as the strange quark effects must originate from disconnected insertions of the $\bar{s} s$ loops from the QCD vacuum [21]. These unquenched ${ }^{\dagger}$ calculations remain time consuming. There have been quenched lattice calculations with a large pion mass computed to date $[22,23]$. Other advancements in lattice QCD have made possible the calculation of both $G_{E}^{s}$ and $G_{M}^{s}$ using low pion masses $(0.3-0.4 \mathrm{GeV})$ combined with chiral extrapolation techniques. These calculations have provided a value of $G_{E}^{s}$ consistent with available experimental data [24] and a result for $G_{M}^{s}=-0.046 \pm 0.019 \mu_{N}[25]$ that is an order of magnitude more precise than that available from experimental measurements.

There are a variety of other calculations, but the disagreements from one model to the next and the unreliability of others only adds to the motivation for constraining these form factors experimentally. HAPPEX-He and $-\mathrm{H}$ provided new limits on these form factors, as is discussed in Chapter 7.

\footnotetext{
${ }^{\dagger}$ In quenched approximations, $\bar{q} q$ loops in the quark sea are neglected. As strange quarks only appear in the proton as loops, a way around this approximation must be found before lattice QCD calculations can add to our understanding of the strange form factors.
} 


\section{$1.2 \quad$ Helicity-Correlated Electron Beam Asymmetries}

My central effort in HAPPEX II-He and HAPPEX II-H involved working to meet the strict experimental requirement of producing a longitudinally polarized electron beam with minimal helicity-correlated differences in its energy, charge asymmetry, position, and angle. These differences (commonly referred to in this thesis as ${ }^{\text {beam }} \mathrm{A}_{\mathrm{LR}}$ 's) can produce false asymmetries in the scattered detector flux leading to a bias in the physics asymmetry. Significant advancements were made in understanding the causes of beam $\mathrm{A}_{\mathrm{LR}}$ 's and in the development of techniques to minimize them. Control over such asymmetries was so impressive that only differences at the level of atomic dimensions were witnessed in the beam parameters between one helicity state and the next. In particular, the source of a large position difference offset caused by a nonzero angle of incidence between the laser beam and the Pockels cell's optic axis was measured and an analytical formalism was developed. Named the "Laser Skew Effect", it is discussed in sections 5.2 and 5.3 of this document. These efforts made possible, in the case of HAPPEX-H, the measurement of the smallest spin asymmetry in the history of electron-nucleon scattering, $-1.6 \pm 0.12$ (stat) \pm 0.05 (syst) parts per million. Additionally, I contributed to the analysis in estimating both the HAPPEX-He and HAPPEX-H background contaminations.

Chapter 2 of this thesis lays out the formalism needed to interpret the HAPPEX-He and HAPPEX-H measurements. Chapter 3 describes the design principles involved in these parity-violation experiments, and Chapter 4 reviews the equipment used to implement them. Chapter 5 describes the polarized electron source along with the sources of and methods 
used to minimize beam $A_{\mathrm{LR}}$ 's. Chapter 6 covers the analysis of the data performed to extract the physics asymmetry and estimates of its uncertainties, and Chapter 7 presents the final results and conclusions of HAPPEX-He and HAPPEX-H and briefly describes related future measurements. In this thesis, I will generally refer to this reported on 2005 run as only "HAPPEX" instead of making the distinction between the "first generation" measurements in 1998 and 1999. Where the distinction is important, I will try to make it. Where it is important to distinguish between the hydrogen and helium measurements, I will try to be clear there as well. 


\section{Chapter 2}

\section{Theory and Formalism}

I present here the formalism needed for interpreting the HAPPEX measurement. The chapter starts with a discussion of electron-nucleon scattering then moves to one about the weak neutral current, and finishes with a discussion of how parity-violation gains access to the weak interaction to reveal a more complete picture of the nucleon's internal structure. Brief sections are interspersed throughout to summarize taking the electron-nucleon formalism to the electron-nucleus formalism needed for electron scattering from helium nuclei, but the central focus will remain on the theoretical approach to parity-violating electron-nucleon scattering.

\section{$2.1 \quad$ Electron-Nucleon Scattering}

Until 1977, the electron had been used solely as an electromagnetic probe. Since then, the use of electron scattering to explore nucleon structure has been done using the weak neutralcurrent at a number of facilities (JLab, SLAC, Bates, Mainz). The weak neutral-current 


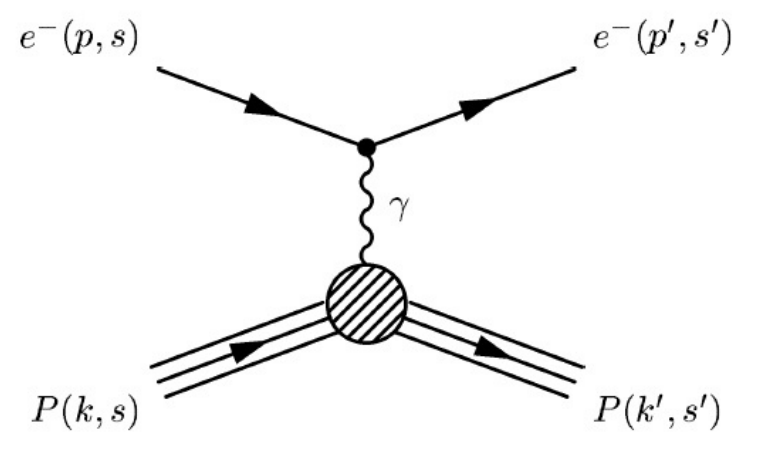

(a) One-photon exchange.

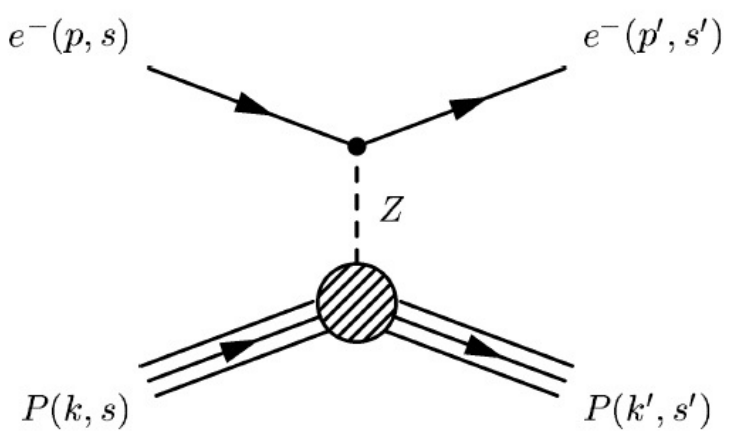

(b) $Z^{0}$ exchange.

Figure 2.1: Feynman diagrams for electron-nucleon scattering.

can be accessed by measuring a parity-violating asymmetry proportional to the interference term between weak and electromagnetic scattering amplitudes. The formalism for understanding this process will be presented in this chapter. Pure electromagnetic scattering will be discussed, including its limitations at revealing certain aspects of nucleon structure. The weak neutral-current will be introduced and how it can offer access to the structure hidden from electromagnetic probes.

\subsubsection{Electromagnetic Scattering}

First we consider elastic electromagnetic scattering of electrons from nucleons, represented in lowest order by the Feynman diagrams in Figure 2.1a. The four-momenta are denoted by $p$ and $p^{\prime}$ for the initial and final electron; $s$ and $s^{\prime}$ are the initial and final electron spin four-vectors. The initial and final four-momenta and spin four-vectors for the proton are, 
respectively $P(k, s)$ and $P\left(k^{\prime}, s^{\prime}\right)$. The amplitude for this scattering process can be written

$$
\mathcal{M}=\frac{1}{q^{2}} j^{\mu} J_{\mu}
$$

where $q^{2}$ is the square of the four-momentum transfer $\left(q=p-p^{\prime}\right.$ for the electron $)$ and

$$
j^{\mu}=-e \bar{u}_{e}\left(p^{\prime}, s^{\prime}\right) \gamma^{\mu} u_{e}(p, s)
$$

is the electron current. The nucleon current $J_{\mu}$ would be written similarly if the nucleon were an elementary particle. Instead $J_{\mu}$ is written in the general form permitted by the necessary restrictions of Lorentz covariance, together with parity and time-reversal invariance [26]:

$$
J^{\mu}=e \bar{u}_{N}\left(k^{\prime}, s^{\prime}\right)\left[\gamma_{\mu} F_{1}\left(q^{2}\right)+\frac{i \sigma_{\mu \nu} q^{\nu}}{2 m_{N}} F_{2}\left(q^{2}\right)\right] u_{N}(k, s)
$$

where $m_{N}$ is the mass of the nucleon and $F_{1}$ and $F_{2}$ are the Dirac and Pauli form factors, respectively, and depend only on $q^{2}$ since this is the only independent scalar variable at the vertex.

It is more common to use linear combinations of $F_{1}$ and $F_{2}$ known as the Sachs form factors

$$
G_{E} \equiv F_{1}-\tau F_{2} \quad \text { and } \quad G_{M} \equiv F_{1}+F_{2}
$$

where $\tau=-q^{2} / 4 m_{N}^{2} . G_{E}$ and $G_{M}$ are referred to as the electric and magnetic form factors, respectively, since for $|\mathbf{q}|^{2} \ll m_{N}^{2}$, when the nucleon does not recoil significantly, $G_{E}\left(q^{2}\right)$ 
and $G_{M}\left(q^{2}\right)$ are the Fourier transforms of the electric and magnetic charge distributions in space. In the limit as $q^{2} \rightarrow 0, G_{E}$ is simply the net electric charge and $G_{M}$ is the net magnetic moment of the nucleon:

$$
G_{E}\left(q^{2}=0\right)=\frac{Q}{e}, \quad G_{M}\left(q^{2}=0\right)=\frac{\mu}{e / 2 m_{N}}
$$

For the proton and neutron specifically, $G_{E}(0)$ and $G_{M}(0)$ are

$$
\begin{array}{ll}
G_{E}^{p}(0)=1, & G_{M}^{p}(0)=\mu_{p}=2.79 \\
G_{E}^{n}(0)=0, & G_{M}^{n}(0)=\mu_{n}=-1.91 .
\end{array}
$$

The mean square charge radius $\left\langle r^{2}\right\rangle$ of the proton is related to the slope of $G_{E}^{p}$ as follows:

$$
\left\langle r^{2}\right\rangle=6\left(\frac{d G_{E}^{p}\left(q^{2}\right)}{d q^{2}}\right)_{q^{2}=0}
$$

as given by the expansion of $G_{E}^{p}$ in orders of $q^{2}$. The evolution of the form factors for $-q^{2}<0$ must be determined experimentally.

The differential cross-section for elastic electron-proton scattering can be calculated using Equation 2.1. The Rosenbluth formula is the result [27]:

$$
\left.\frac{d \sigma}{d \Omega}\right|_{l a b}=\frac{\alpha^{2}}{4 E^{2} \sin ^{4}(\theta / 2)} \frac{E^{\prime}}{E}\left(\frac{\left(G_{E}^{p}\right)^{2}+\tau\left(G_{M}^{p}\right)^{2}}{1+\tau} \cos ^{2} \frac{\theta}{2}+2 \tau\left(G_{M}^{p}\right)^{2} \sin ^{2} \frac{\theta}{2}\right) .
$$

where $\alpha$ is the fine structure constant, $E$ and $E^{\prime}$ are, respectively, the incident and scattered electron energy, and $\theta$ is the lab scattering angle. Using the Sachs form factors leaves no 
cross terms in the cross section expression and allows for experimental determination of the form factors by the Rosenbluth separation method. The proton and neutron form factors have been measured over a wide range of $Q^{2}$ values [28]. Traditionally, the $Q^{2}$ dependence of the form factors has been parameterized by a dipole and Galster form [29] and more recently with a phenomenological fit [30] and a fit using polynomials [31]. Replacing the $p$ with an $n$ in Equation 2.7 presents the corresponding cross section for the neutron.

One other feature of note is the $\sin ^{-4}(\theta / 2)$ dependence of the cross section. At small scattering angles, there is a large variation of the scattering rate into a particular solid angle for small variations in the beam's position and angle on the target. A large portion of the work in the thesis is motivated by this strong angular dependence at small scattering angles.

\subsubsection{Electron-Nucleus Scattering}

The formalism for electron-nucleus scattering is needed for helium, and a multipole analysis reveals a form of the cross section that allows for transitions to discrete states. More extensive detail related to this can be found in Ref $[26,32,33]$. This method expands hadronic currents in terms of multipole projections of the charge and three-current operators.

The process through which this formalism is developed is as follows: spatial dependence is restored to the nucleon current $J^{\mu}$ to provide the charge density operator $\hat{\rho}(\mathbf{x})$, spherical unit vectors are inserted to provide the three-current operator $\hat{\mathbf{J}}(\mathbf{x})$, the plane waves $\left(e^{i \mathbf{q} \cdot \mathbf{x}}\right)$ are expanded in terms of Bessel functions and spherical harmonics to create a differential cross section in terms of nuclear matrix elements, and if good initial and final state quantum numbers are assumed, the electromagnetic form factors can then be defined in terms of reduced matrix elements in both angular momentum and isospin. The total form factor $F^{2}$ 
is defined in terms of the longitudinal and transverse contributions

$$
F^{2}(q, \theta)=v_{L} \sum_{J \geq 0} F_{C J}^{2}(q)+v_{T} \sum_{J \geq 1}\left[F_{E J}^{2}(q)+F_{M J}^{2}(q)\right]
$$

where $v_{L}$ and $v_{T}$ are the longitudinal and transverse lepton kinematical factors, and $F_{C J}^{2}$, $F_{E J}^{2}$, and $F_{M J}^{2}$ are, respectively, the charge, electric, and magnetic form factors.

\subsubsection{Neutral Weak Form Factors}

Electron-nucleon scattering can also proceed through the exchange of a virtual $Z^{0}$ boson, represented in the lowest order by the Feynman diagram in Figure 2.1. This exchange gives rise to a new current, the weak neutral current $J_{\mu}^{Z}$. The electromagnetic current is now labelled $J_{\mu}^{\gamma}$ to avoid confusion.

The amplitude for neutral weak electron-nuceon scattering is written

$$
\mathcal{M}=\frac{G_{F}}{\sqrt{2}} j^{Z \mu} J_{\mu}^{Z}
$$

where $G_{F}$ is the Fermi coupling constant, the electron current is

$$
j^{Z \mu}=\bar{u}_{e} \gamma^{\mu}\left(c_{V}^{e}-c_{A}^{e} \gamma^{5}\right) u_{e}
$$

and, the nucleon current:

$$
J_{\mu}^{Z}=\bar{u}_{N}\left[\gamma_{\mu} F_{1}^{Z}\left(q^{2}\right)+\frac{i \sigma_{\mu \nu} q^{\nu}}{2 M_{N}} F_{2}^{Z}\left(q^{2}\right)+\gamma_{\mu} \gamma_{5} G_{A}^{Z}\right] u_{N}
$$

There are three new form factors introduced here, all dependent on $q^{2} ; F_{1}^{Z}$ and $F_{2}^{Z}$ are the 


\begin{tabular}{|crcr|}
\hline Particle & $q^{e m}$ & $g^{V}$ & $g^{A}$ \\
$e^{-}$ & -1 & $-\frac{1}{4}+\sin ^{2} \theta_{W}$ & $\frac{1}{4}$ \\
$u$ & $\frac{2}{3}$ & $\frac{1}{4}-\frac{2}{3} \sin ^{2} \theta_{W}$ & $-\frac{1}{4}$ \\
$d, s$ & $-\frac{1}{3}$ & $-\frac{1}{4}+\frac{1}{3} \sin ^{2} \theta_{W}$ & $\frac{1}{4}$ \\
\hline
\end{tabular}

Table 2.1: Electromagnetic and weak charges for the electron and light quarks.

weak neutral form factors and are analogous to $F_{1}^{\gamma}$ and $F_{2}^{\gamma}$, the electromagnetic form factors. The relationship between the weak and electromagnetic form factors will be discussed in the next section. There is also an axial form factor $G_{A}^{Z}$ in the nucleon current, but it contributes little at HAPPEX's kinematics. It is most important at backward angles.

The charges for the electromagnetic and weak neutral currents are summarized in Table 2.1. $q^{E M}$ is the particle's electromagnetic charge, and $g^{V}$ and $g^{A}$ are the weak axial and weak vector charges. The right- and left-helicity charges are $g^{R}=g^{V}+g^{A}$ and $g^{L}=g^{V}-g^{A}$, respectively. The electromagnetic and weak charges are of comparable size, yet the weak interaction cross section is suppressed by a factor of $\sim 10^{9}$ at $Q^{2}=0.1$ due to a factor of $Q^{2} / M_{Z}^{2}$ at each vertex.

\subsubsection{Relationship Between Electromagnetic and Weak Form Factors}

In a QCD inspired description of electron-nucleon scattering, the gauge bosons couple to the quarks in the nucleon, and the nucleon vector currents $J_{\mu}^{\gamma}$ and $J_{\mu}^{Z}$ can be broken up into a sum of currents for each quark flavor. The relationship between the electromagnetic and weak form factors can be established by considering these quark currents.

First, the nucleon vector currents $J_{\mu}^{\gamma}$ and $J_{\mu}^{Z}$ can be written as nucleonic matrix elements 
of the quark current operators $\hat{J}_{\mu}^{\gamma}$ and $\hat{J}_{\mu}^{Z}$,

$$
J_{\mu}^{\gamma} \equiv\left\langle N\left|\hat{J}_{\mu}^{\gamma}\right| N\right\rangle, \quad J_{\mu}^{Z} \equiv\left\langle N\left|\hat{J}_{\mu}^{Z}\right| N\right\rangle
$$

where $\langle N|$ represents the state of a nucleon and the quark current operators are written as

$$
\hat{J}_{\mu}^{\gamma} \equiv \sum_{q} Q_{q} \bar{q} \gamma_{\mu} q, \quad \hat{J}_{\mu}^{Z} \equiv \sum_{q} g_{q}^{V} \bar{q} \gamma_{\mu} q
$$

where $Q_{q}$ and $g_{q}^{V}$ are the electromagnetic and neutral weak vector quark couplings for the Standard Model, respectively, and the summations extend over all quark flavors $q$. The nucleon currents can be written down explicitly in terms of the quark-nucleon currents as:

$$
\begin{aligned}
& J_{\mu}^{\gamma}=\left\langle N\left|\hat{J}_{\mu}^{\gamma}\right| N\right\rangle= \frac{2}{3}\left\langle N\left|\bar{u} \gamma_{\mu} u\right| N\right\rangle-\frac{1}{3}\left\langle N\left|\bar{d} \gamma_{\mu} d\right| N\right\rangle-\frac{1}{3}\left\langle N\left|\bar{s} \gamma_{\mu} s\right| N\right\rangle \\
& J_{\mu}^{Z}=\left\langle N\left|\hat{J}_{\mu}^{Z}\right| N\right\rangle=\left(\frac{1}{4}-\frac{2}{3} \sin ^{2} \theta_{W}\right)\left\langle N\left|\bar{u} \gamma_{\mu} u\right| N\right\rangle \\
&+\left(-\frac{1}{4}+\frac{1}{3} \sin ^{2} \theta_{W}\right)\left\langle N\left|\bar{d} \gamma_{\mu} d\right| N\right\rangle \\
&+\left(-\frac{1}{4}+\frac{1}{3} \sin ^{2} \theta_{W}\right)\left\langle N\left|\bar{s} \gamma_{\mu} s\right| N\right\rangle .
\end{aligned}
$$

As it is assumed that only $u, d, s$, quarks contribute the nucleon's properties, only terms pertaining to those flavors are included above in the nucleon current expressions. The same quark-nucleon currents are present in both Equations 2.13 and 2.14 as these currents depend only on the vector nature of the coupling, not on whether the coupling proceeds through the exchange of a photon or $Z^{0}$ boson. This fact will allow us to relate the electromagnetic, neutral weak, and strange form factors. 
Each of the electromagnetic and neutral weak form factors introduced in Sections 2.1.1 and 2.1.3 are associated with a particular nucleon meaning there are eight nucleon form factors which can be written down compactly as $F_{(1,2)}^{(p, n)(\gamma, Z)}$. The quark-nucleon currents of the different nucleon varieties can be written in the most general form by introducing quark-nucleon form factors defined by

$$
\left\langle N\left|\bar{q} \gamma_{\mu} q\right| N\right\rangle \equiv \bar{u}_{N}\left[\gamma_{\mu} F_{1}^{N q}+\frac{i \sigma_{\mu \nu} q^{\nu}}{2 m_{N}} F_{2}^{N q}\right] u_{N}
$$

where $q=u, d$, or $s$ and $N=p$ or $n$.

Writing the electromagnetic nucleon and quark-nucleon currents of Equation 2.13 in terms of form factors,

$$
J_{\mu}^{\gamma}=Q \bar{u}_{N}\left[\gamma_{\mu} F_{1}^{N \gamma}+\frac{i \sigma_{\mu \nu} q^{\nu}}{2 m_{N}} F_{2}^{N \gamma}\right] u_{N}=\sum_{q=u, d, s} Q_{q} \bar{u}_{N}\left[\gamma_{\mu} F_{1}^{N q}+\frac{i \sigma_{\mu \nu} q^{\nu}}{2 m_{N}} F_{2}^{N q}\right] u_{N}
$$

it is apparent that the nucleon electromagnetic form factors may be expressed as sums of the quark-nucleon form factors with Standard Model quark couplings:

$$
F_{(1,2)}^{N \gamma}=\sum_{q=u, d, s} Q_{q} F_{1,2}^{N q}=\frac{2}{3} F_{1,2}^{N u}-\frac{1}{3} F_{1,2,}^{N d}-\frac{1}{3} F_{1,2,}^{N s}
$$

Similarly, the nucleon neutral weak vector form factors may be expressed:

$$
F_{1,2}^{N Z}=\sum_{q=u, d, s} g_{q}^{V} F_{1,2}^{N q}
$$

The Sachs form factors (defined in Equation 2.4) have corresponding relationships. In 
paticular, $G_{E, M}^{p \gamma}, G_{E, M}^{n \gamma}$, and $G_{E, M}^{p Z}$ are related to the quark form factors $G^{u}, G^{d}$, and $G^{s}$ as

$$
\begin{gathered}
G_{E, M}^{p \gamma}=\frac{2}{3} G_{E, M}^{p u}-\frac{1}{3} G_{E, M}^{p d}-\frac{1}{3} G_{E, M}^{p s}, \\
G_{E, M}^{n \gamma}=\frac{2}{3} G_{E, M}^{n u}-\frac{1}{3} G_{E, M}^{n d}-\frac{1}{3} G_{E, M}^{n s}, \\
G_{E, M}^{p Z}=\left(\frac{1}{4}-\frac{2}{3} \sin ^{2} \theta_{W}\right) G_{E, M}^{p u}+\left(-\frac{1}{4}+\frac{1}{3} \sin ^{2} \theta_{W}\right) G_{E, M}^{p d}+\left(-\frac{1}{4}+\frac{1}{3} \sin ^{2} \theta_{W}\right) G_{E, M}^{p s} .
\end{gathered}
$$

A second relationship between the Sachs and quark form factors is gained by using charge symmetry [34], a restricted form of $\mathrm{SU}(2)$ isospin symmetry between up and down quarks*. Charge symmetry asserts invariance under the following interchanges: $p \leftrightarrow n, u \leftrightarrow d, s \leftrightarrow s$ and provides the additional relation

$$
G_{E, M}^{n \gamma}=\frac{2}{3} G_{E, M}^{n d}-\frac{1}{3}\left(G_{E, M}^{n u}+G_{E, M}^{n s}\right)
$$

Any violations of charge symmetry are expected to be small and will alter the electromagnetic form factors by less than $1 \%$ [34]. These identities allow us to eliminate the up and down form factors in favor of the proton and neutron electromagnetic form factors to obtain

$$
G_{E, M}^{p Z}=\left(\frac{1}{4}-\sin ^{2} \theta_{W}\right) G_{E, M}^{p \gamma}-\frac{1}{4} G_{E, M}^{n \gamma}-\frac{1}{4} G_{E, M}^{s}
$$

This is the desired relationship among the form factors as $G_{E, M}^{p \gamma}$ and $G_{E, M}^{n \gamma}$ are known from previous experiments; the strange quark form factors $G_{E, M}^{s}$ may then be determined by measuring the proton's neutral weak from factor $G_{E, M}^{p Z}$.

\footnotetext{
*If one neglects the mass difference between up and down quarks and ignores the electromagnetic effects, the QCD Lagrangian is invariant under exchange of up and down quarks. This invariance is called charge symmetry and is a specific rotation in isospin space making it more restrictive than $\mathrm{SU}(2)$ isospin symmetry, which is invariant under any rotation in isospin space.
} 


\subsection{Parity Violation}

The proton's neutral weak form factor cannot be measured directly as the amplitude for $Z^{0}$ exchange is dominated by the amplitude for electromagnetic scattering. The neutral weak amplitude may be accessed instead by using parity violation to measure an asymmetry $A_{L R}$ that arises from an interference term in the cross section between the electromagnetic and weak scattering amplitudes, $\mathcal{M}_{Z}$ and $\mathcal{M}_{\gamma}$ respectively. The total scattering amplitude is expressed as a sum between the electromagnetic and weak amplitudes, and the cross sections for right- and left-handed electrons scattering from an unpolarized target are proportional to the square of the total amplitudes,

$$
\sigma_{R} \propto\left(\mathcal{M}_{\gamma}+\mathcal{M}_{Z}^{R}\right)^{2} \quad \sigma_{L} \propto\left(\mathcal{M}_{\gamma}+\mathcal{M}_{Z}^{L}\right)^{2}
$$

As the weak interaction violates parity, the weak scattering amplitude $\mathcal{M}_{Z}$ depends upon the helicity of the incident electron resulting in different cross sections for right- and lefthanded electrons. This difference is usually expressed as an asymmetry $A_{L R}$ defined as

$$
A_{L R} \equiv \frac{\sigma_{R}-\sigma_{L}}{\sigma_{R}+\sigma_{L}}
$$

and in terms of the scattering amplitudes, becomes (assuming $\mathcal{M}_{Z} \ll \mathcal{M}_{\gamma}$ ):

$$
A_{L R}=\frac{\left(\mathcal{M}_{\gamma}+\mathcal{M}_{Z}^{R}\right)^{2}-\left(\mathcal{M}_{\gamma}+\mathcal{M}_{Z}^{L}\right)^{2}}{\left(\mathcal{M}_{\gamma}+\mathcal{M}_{Z}^{R}\right)^{2}+\left(\mathcal{M}_{\gamma}+\mathcal{M}_{Z}^{L}\right)^{2}} \approx \frac{\mathcal{M}_{Z}^{R}-\mathcal{M}_{Z}^{L}}{M_{\gamma}}
$$


The weak-electromagnetic interference gives rise to a parity-violating asymmetry that is essentially the ratio of amplitudes instead of a ratio of the square of amplitudes. This asymmetry provides access to a measurable quantity that is directly proportional to the weak neutral current.

A standard calculation [26] for the asymmetry yields an expression in terms of the electromagnetic and neutral weak form factors:

$$
A_{L R}=-\frac{G_{F} Q^{2}}{4 \pi \alpha \sqrt{2}}\left[\frac{\epsilon G_{E}^{p \gamma} G_{E}^{p Z}+\tau G_{M}^{p \gamma} G_{M}^{p Z}-\frac{1}{2}\left(1-4 \sin ^{2} \theta_{W}\right) \epsilon^{\prime} G_{M}^{p \gamma} G_{A}^{p Z}}{\epsilon\left(G_{E}^{p \gamma}\right)^{2}+\tau\left(G_{M}^{p \gamma}\right)^{2}}\right]
$$

where $G_{E, M}^{p \gamma}$ are the electric and magnetic Sachs form factors due to photon exchange, $G_{E, M}^{p Z}$ and $G_{A}^{p Z}$ are the vector and axial from factors for $Z^{0}$ exchange, $Q^{2} \equiv-q^{2}$ is the four-momentum transfer squared, and $\epsilon, \tau$, and $\epsilon^{\prime}$ are the kinematic quantities defined as

$$
\epsilon \equiv \frac{1}{1+2\left|\mathbf{q}^{2} / Q^{2}\right| \tan ^{2}(\theta / 2)}, \quad \tau \equiv \frac{Q^{2}}{4 m_{p}^{2}}, \text { and } \epsilon^{\prime} \equiv \sqrt{\tau(1+\tau)\left(1-\epsilon^{2}\right)}
$$

This asymmetry can be rewritten using the known form factors $G_{E, M}^{p \gamma}, G_{E, M}^{n \gamma}$, and the strange form factors $G_{E, M}^{s}$ we are trying to measure by inserting Equation 2.20 into Equation 2.24. Upon this substitution, $A_{L R}$ becomes

$$
\begin{aligned}
A_{L R}= & {\left[-\frac{G_{F} Q^{2}}{4 \pi \alpha \sqrt{2}}\right]\left\{\left(1-4 \sin ^{2} \theta_{W}\right)-\frac{\epsilon G_{E}^{p \gamma}\left(G_{E}^{n \gamma}+G_{E}^{s}\right)+\tau G_{M}^{p \gamma}\left(G_{M}^{n \gamma}+G_{M}^{s}\right)}{\epsilon\left(G_{E}^{p \gamma}\right)^{2}+\tau\left(G_{M}^{p \gamma}\right)^{2}}\right.} \\
& \left.-\frac{\left(1-4 \sin ^{2} \theta_{W}\right) \sqrt{\tau(1+\tau)} \sqrt{\left(1-\epsilon^{2}\right)} G_{M}^{p \gamma} G_{A}^{p Z}}{\epsilon\left(G_{E}^{n \gamma}\right)^{2}+\tau\left(G_{M}^{n \gamma}\right)^{2}}\right\}
\end{aligned}
$$

This asymmetry is quite sensitive to the linear combination of $G_{E}^{s}$ and $G_{M}^{s}$. There is little 
sensitivity to the neutral weak axial form factor $G_{A}^{p Z}$ due to the factor $\left(1-4 \sin ^{2} \theta_{W}\right) \ll 1$ and can be neglected in our case.

The relative contribution of the strange form factors depends on the kinematics. At forward angles where $\epsilon$ is large, $A_{L R}$ 's sensitivity to $G_{E}^{s}$ is maximized; some contribution from $G_{M}^{s}$ appears at all angles although it is maximized at backward angles. An interesting case appears at forward angles as $\tau \rightarrow 0$. Here, the asymmetry becomes independent of strange form factors since $G_{E}^{s}(0)=0$ making elastic scattering from hydrogen a test of the Standard Model [36].

An extension parity-violation effort has been developed at three accelerator facilities to measure the strange quark form factors. The SAMPLE experiment at MIT-Bates measures $G_{M}^{s}$ and $G_{A}^{Z p}$ at backward angles from hydrogen and deuterium targets, respectively, at $Q^{2}=0.1 \mathrm{GeV}^{2}[37,38]$. The A4 experiment at the Mainz Microtron measured a linear combination of $G_{E}^{s}$ and $G_{M}^{s}$ at forward angles from hydrogen at $Q^{2}=0.1$ and $0.22 \mathrm{GeV}^{2}$. The G0 experiment at Jefferson Lab measured linear combinations of $G_{E}^{s}$ and $G_{M}^{s}$ over the range $0.12<Q^{2}<0.8 \mathrm{GeV}^{2}$ at forward angles from a hydrogen target and $G_{M}^{s}$ and $G_{A}^{Z p}$ at backward angles from hydrogen and deuterium targets at $Q^{2}=0.23$ and $0.6 \mathrm{GeV}^{2}$. And, finally the HAPPEX collaboration measured a linear combination of $G_{E}^{s}$ and $G_{M}^{s}$ at $Q^{2}=0.1,0.48$, and $0.6 \mathrm{GeV}^{2}$ at forward angles from hydrogen target and $G_{E}^{s}$ from a helium target. 


\subsection{Spinless Isoscalar Nuclei}

An asymmetry for the ${ }^{4} \mathrm{He}$ measurement, where electrons are elastically scattered from a spinless isoscalar target, can be derived in terms of $G_{E, M}^{p \gamma}, G_{E, M}^{n \gamma}$, and $G_{E, M}^{s}$. Needed, however, is the additional assumption that the nuclear form factors are a product of the nucleon form factor and another form factor arising from the shape of the nucleus. The result is [35]

$$
A_{L R}=\frac{G_{F} Q^{2}}{\pi \alpha \sqrt{2}}\left[\sin ^{2} \theta_{W}+\frac{G_{E}^{s}}{2\left(G_{E}^{p}+G_{E}^{n}\right)}\right] .
$$

As expected for a spinless isoscalar target, the asymmetry is independent of $G_{M}^{s}$. The axial form factor $G_{A}^{s}$ is also not present. This measurement is sensitive to $G_{E}^{s}$ and there are models [39] suggesting a larger value for it at the lower $Q^{2}$ range around $\sim 0.1 \mathrm{GeV}^{2}$. The isoscaler nucleus ${ }^{4} \mathrm{He}$ is favored over ${ }^{12} \mathrm{C}$ as the corrections for its nuclear structure are better understood. Contributions to this asymmetry due to isospin mixing [40] and $D$ state admixtures [41] are negligible in the ${ }^{4} \mathrm{He}$ ground state, as are meson-exchange current contributions at the low $Q^{2}[44]$ of this measurement. The ${ }^{4} \mathrm{He}$ measurement is used along with the hydrogen measurement to cleanly separate $G_{E}^{s}$ and $G_{M}^{s}$. 


\section{Chapter 3}

\section{Experimental Design}

\subsection{Overview}

The second generation Hall A Proton Parity Experiment (HAPPEX) at Jefferson Lab uses the weak interaction via parity-violating electron scattering from both ${ }^{4} \mathrm{He}$ and ${ }^{1} \mathrm{H}$ to further constrain measurements of the electric and magnetic strange form factors $G_{E}^{s}$ and $G_{M}^{s}$. The first generation of HAPPEX measured a parity-violating asymmetry of $~ 15$ ppm at $Q^{2}=0.48 \mathrm{GeV}^{2}$ off hydrogen with a relative error of $6 \%$ [45]. This experiment made a $\sim 1.5 \mathrm{ppm}$ measurement scattering off ${ }^{1} \mathrm{H}$ and one of $\sim 8 \mathrm{ppm}$ with ${ }^{4} \mathrm{He}$ at $Q^{2} \sim 0.1 \mathrm{GeV}^{2}$ with a relative error of $\sim 10 \%$. The difference in hydrogen asymmetries suggests needed improvement in control over systematic effects by an order of magnitude for this HAPPEX II run compared to earlier runs.

The measurement at $Q^{2}=0.1 \mathrm{GeV}^{2}$ requires observing the elastically scattered electrons at very forward angles of $6^{\circ}$ at an energy of $3 \mathrm{GeV}$. A high precision measurement is possible in a relatively short time at these kinematics for both ${ }^{4} \mathrm{He}$ and ${ }^{1} \mathrm{H}$. 


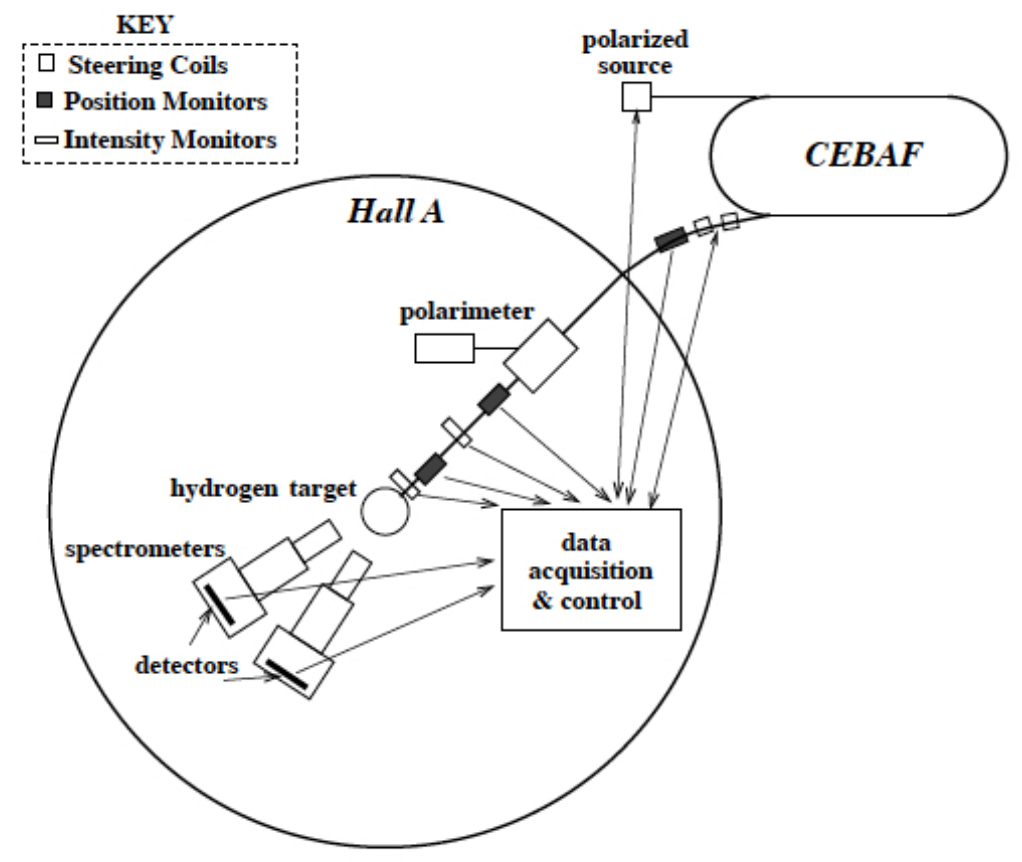

Figure 3.1: Overview of the experimental apparatus

The measured asymmetry is proportional to the product of the beam polarization and the parity-violating asymmetry meaning that a high beam polarization decreases the amount of data taking necessary to achieve the same statistical precision. The HAPPEX II collaboration took advantage of Jefferson Lab's high beam polarization $(>80 \%)$ and high current $(>35 \mu \mathrm{A})$ to achieve impressive statistical precision on such small asymmetries. Such precision also means that systematic errors must be kept far smaller. The following sections of this chapter outline how small asymmetries were cleanly measured and the methods used to control systematic errors well below the level of statistical precision. 


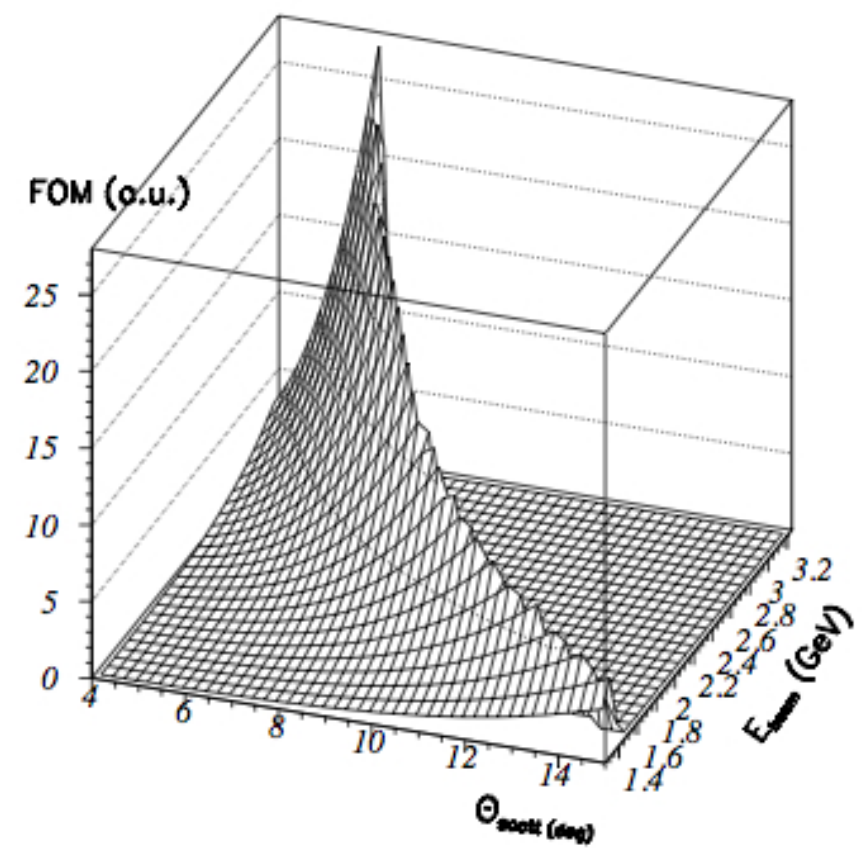

Figure 3.2: Figure of Merit for the measurement of strange form factors in elastic electron-proton scattering. Only the kinematical domain $Q^{2}<0.25 \mathrm{GeV}^{2}$ is represented.

\subsection{Choice of Kinematics}

The choice of running conditions was driven by our sensitivity to the linear combination of $G_{E}^{s}$ and $G_{M}^{s}$ in the case of hydrogen, and $G_{E}^{s}$ in the case of helium. The quantity $A^{2} d \sigma / d \Omega$ where $A$ is the asymmetry and $d \sigma / d \Omega$ is the differential cross section at a given kinematic point, is known as the Figure of Merit for an asymmetry measurement. It is a measure of the statistical significance achievable in a given amount of time. We wanted to maximize the Figure of Merit in order to minimize the fractional statistical error $\Delta A / A$ of the measured asymmetry.

Figure 3.2 shows the Figure of Merit for the hydrogen measurement as a function of the two free parameters of the elastic kinematics, the scattering angle and the beam energy. 
The plotted quantity is the square of $\delta\left(\rho_{s}+\mu_{p} \mu_{s}\right)^{-1}$ [42] where, with $\tau=Q^{2} / 4 m_{N}^{2}$,

$$
\left.\rho_{s} \equiv \frac{d G_{E}^{s}}{d \tau}\right|_{\tau=0} \quad \text { and } \quad \mu_{s} \equiv G_{M}^{s}(0)
$$

are the strangeness radius and strange magnetic moment, respectively. It is often more convenient to talk about a strangeness Dirac radius, in analogy to the charge radius $\left\langle r^{2}\right\rangle$ in Equation 2.6, which is related to the above quantities as follows:

$$
\left\langle r_{s}^{2}\right\rangle \equiv-6 \frac{d F_{1}^{s 2}}{d Q^{2}} ; \quad \rho_{s}=-\frac{2}{3} M_{p}^{2}\left\langle r_{s}^{2}\right\rangle-\mu_{s}
$$

Here, $F_{1}^{s}$ is the Dirac strange form factor and $M_{P}$ is the nucleon mass [42]. To achieve an accurate measurement of $\left\langle r_{s}^{2}\right\rangle$ to better than \pm 0.05 , we wished to go sufficiently low in $Q^{2}\left(\sim 0.1 \mathrm{GeV}^{2}\right)$ so that information on the leading moments of $\rho_{s}$ and $\mu_{s}$ could be obtained with little dependence of the $Q^{2}$ behavior of the form factors. The figure shows at very low $Q^{2}$ (small $\theta$ and low beam energy) that the sensitivity to strangeness in the nucleon falls off. The running conditions selected in the 1999 HAPPEX-H proposal [42] were a scattering angle $6^{\circ}$ and a beam energy of $3.2 \mathrm{GeV}$. These conditions suggested that a statistical accuracy of $4.6 \%$ was achievable in 700 hours of beam running time*.

For the helium measurement, with the mean scattering angle at $6^{\circ}$, our sensitivity to $\rho_{s}$ was maximized around a incident electron energy of $3.4 \mathrm{GeV}$ and $Q^{2} \sim 0.1 \mathrm{GeV}^{2}$ (shown by the broad peak on the Figure of Merit plot in Figure 3.3). This, fortuitously, is very nearly the same $Q^{2}$ as planned for the hydrogen run. At these running conditions, a 700 hour beam

${ }^{*}$ This estimate assumed a beam current of $100 \mu \mathrm{A}$ and a beam polarization of $75 \%$. During the hydrogen run, the beam current was only between $35-40 \mu \mathrm{A}$ and the beam polarization was $\sim 86 \%$. 


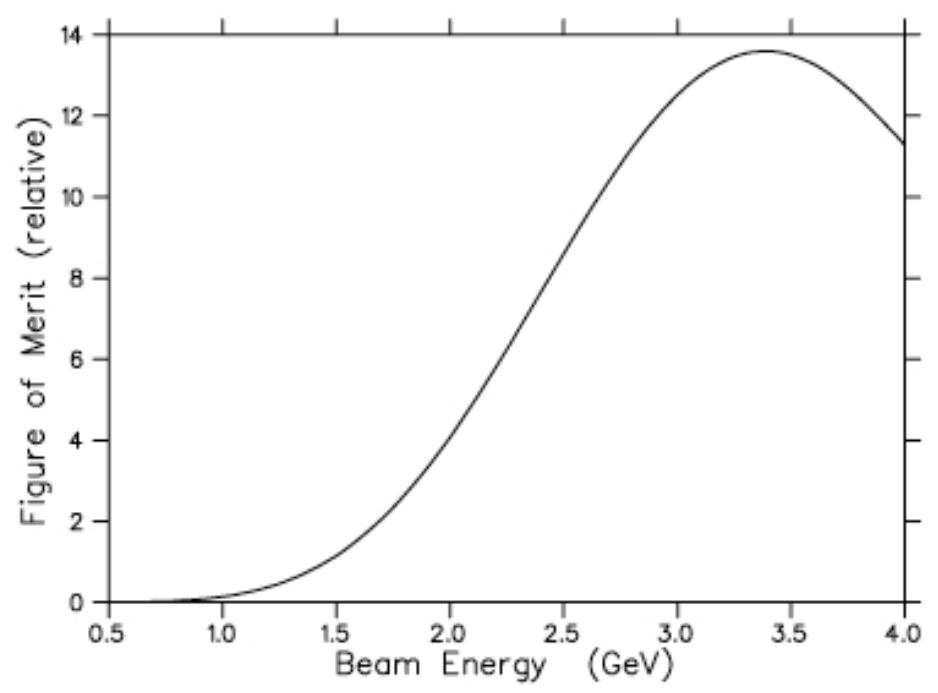

Figure 3.3: Relative Figure of Merit as a function of beam energy, at a $6^{\circ}$ scattering angle

run would have had an overall $2.2 \%$ statistical error $^{\dagger}[43]$.

\subsection{Experimental Technique}

The HAPPEX II experiments will scatter longitudinally polarized electrons from unpolarized protons in a liquid hydrogn target and unpolarized ${ }^{4} \mathrm{He}$ nuclei in a gaseous target. The parity-violating asymmetry in each case is defined as

$$
A_{P V}=\frac{\sigma_{R}-\sigma_{l}}{\sigma_{R}+\sigma_{L}}
$$

where $\sigma_{R}\left(\sigma_{L}\right)$ is the cross-section for electrons of right (left) helicity. Electrons with spins aligned parallel (antiparallel) to the beam direction are defined as right (left) helicity.

What is measured in these experiments is a quantity linearly proportional to the cross-

\footnotetext{
${ }^{\dagger}$ This estimate assumed $100 \mu \mathrm{A}$ of beam current, $80 \%$ polarized beam, and a small Al cell wall background error correction [43]. The beam current during the helium measurement actually ranged from 30-35 $\mu \mathrm{A}$, the beam polarization was $\sim 83 \%$, and the Al cell correction was small as expected.
} 
sections; this implies that any common scale factor will cancel when calculating this asymmetry. What both experiments measure is the normalized detector flux, which is the detector flux normalized to the beam current. This flux is integrated over $\sim 33$ ms windows that flip rapidly between right and left helicity states. Right and left windows that fall next to each other have their signals subtracted to form a single parity-violating asymmetry measurement, referred to as a single "window pair". 25.3 million such pairs were recorded for ${ }^{1} \mathrm{H}$ and 34.1 million for ${ }^{4} \mathrm{He}$. The following sections will discuss in further detail how this was done.

\subsection{Fast Helicity Reversal}

Our asymmetry measurements could be very sensitive to slow drifts in experimental parameters (e.g., beam energy, position, intensity). To minimize our sensitivity to such drifts, we rapidly reversed the helicity of the beam so that any beam parameter drifts would be experienced equally by both helicity states. The helicity was reversed at $30 \mathrm{~Hz}$ in a pairrandom sequence as shown in Figure 3.2. The first $33 \mathrm{~ms}$ "window" of each pair was chosen pseudorandomly by a shift-register algortihm [46], and the following window was always the complement. The helicity was reversed randomly so as to prevent periodic noise from correlating with the beam's helicity.

Polarized electrons of different helicity states were produced by photoemission from a Gallium Arsenide (GaAs) photocathode. As the cathode was illuminated with circularly polarized light, electrons were preferentially excited (depending on the sign of the light's polarization) from the valence band $\left(P_{3 / 2}\right)$ to one of the two $s$-states in the conduction band $\left(S_{1 / 2}\right)$. The helicity of the excited electron depends on whether the incoming laser 


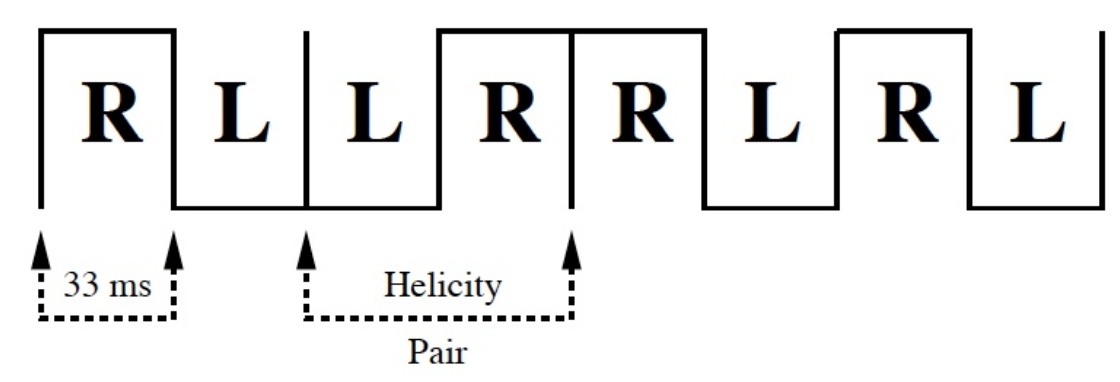

Figure 3.4: A pseudorandom helicity sequence

polarization is left or right circularly polarized, and this depends on whether the helicity sequence input to the high-voltage (HV) switch of an electro-optical device called a Pockels cell is positive or negative. The Pockels cell acts as a quarter-wave plate which converts linear light into right or left circularly polarized light, and it flips the light's helicity at a rate of $30 \mathrm{~Hz}$.

\subsection{Integration}

To make a measurement of a $1 \mathrm{ppm}$ quantity to a precision of $10 \%$, counting statistics require on the order of $10^{14}$ electrons. To do this in a reasonable period of time by counting individual events, a highly segmented detector would be needed to reach the required detector rate. It was decided instead to integrate the detected flux using a total absorption Cerenkov calorimeter. Using the integration technique means that there is no detector deadtime thereby avoiding possibly large helicity-correlated corrections. This technique is, however, sensitive to the linearity of the detector PMTs (discussed in Section 6.1.3) and background contributions which are present in the flux into the detector (discussed in Section 3.10). At the end of a helicity "window", the charge stored in a capacitor over $33 \mathrm{~ms}$ is 
discharged and is then digitized by an ADC.

\subsection{Statistical Error}

To measure the normalized flux, we integrate the analog signals from two detectors and the beam current monitor over the duration of each helicity window. This normalized flux is given by the ratio of the integrated detector signal $D$ to the integrated current monitor signal $I$. The raw asymmetry for each window pair is computed by:

$$
A_{\text {pair }}=\frac{\sigma_{R}-\sigma_{L}}{\sigma_{R}+\sigma_{L}}=\frac{D_{R} / I_{R}-D_{l} / I_{L}}{D_{R} / I_{R}+D_{L} / I_{L}}
$$

The statistical error on the asymmetry from a single pair is, in principle, due to the counting statistics of the experiment. If $N_{R}$ and $N_{L}$ are the number of electrons for rightand left-helicity pulses, respectively, then the statistical error for a single pair is

$$
1 / \sqrt{N_{R}+N_{L}}
$$

The RMS width of a distribution of asymmetry pairs is equal to the statistical error on a single pair. In practice, counting statistics on a single pair gives the minimum RMS width that distribution can have. Other sources of noise can broaden pulse-pair asymmetry distributions. Instrument noise in the beam position and beam current monitors, ADC bit-resolution, fluctuations in detector pedestals, limited energy resolution of the detector (discussed in Section 4.5.5), and target density fluctuations (discussed in Section 4.4) are among them. These sources are generally random and therefore derive from a normal distri- 
bution. They add in quadrature with counting statistics to determine the statistical error. ${ }^{\ddagger}$ To minimize the running time required to make a measurement, parity-violating experiments are designed such that contributions to the statistical error from sources other than counting statistics are held well below the level of counting statistics. The largest source of these contributions during the experiment came from detector energy resolution and target density fluctuations, each contributing $<2 \%$ of the RMS demanded by counting statistics.

The elastic scattering rates were about $12 \mathrm{~Hz}$ for ${ }^{4} \mathrm{He}$ and $120 \mathrm{~Hz}$ for ${ }^{1} \mathrm{H}$ giving theoretical measurements of the asymmetries based upon counting statistics of $0.11 \%$ and $0.05 \%$, respectively. The asymmetry was measured 35 million times for ${ }^{4} \mathrm{He}$ and 25 million times for ${ }^{1} \mathrm{H}$ giving absolute measurements to the asymmetries of $0.18 \mathrm{ppm}$ and $0.08 \mathrm{ppm}$, respectively.

\section{Estimate of Rate}

The scattering rate into the detector can be estimated with the help of Equation 2.7. At a beam energy of $3.2 \mathrm{GeV}$, a $Q^{2}$ of $0.11 \mathrm{GeV}^{2}$, and a mean scattering angle of $6^{\circ}$, the differential cross section for scattering from hydrogen is

$$
\frac{d \sigma}{d \Omega}=44.83 \text { microbarns/steradian }(\mu \mathrm{barn} / \mathrm{sr})
$$

The number of electrons $N_{s}$ scattered into each spectrometer's acceptance can be expressed

as

$$
N_{s}=\frac{d \sigma}{d \Omega} \cdot I \cdot \rho \cdot L \cdot \Delta \Omega \cdot f_{b}
$$

\footnotetext{
${ }^{\ddagger}$ In general, physicists tend to equate "statistics" with "counting statistics". However, in parity-violating experiments, the term "statistics" is used differently to refer to the width of a particular distribution. "Counting statistics" then refers to the minimum value that width can take.
} 
where $I$ is the beam current, $\rho$ is the target density, $L$ is the target length, $\Delta \Omega$ is the solid angle acceptance of each spectrometer, and $f_{b}$ is a correction factor to account for particles lost due to Bremsstrahlung in the target. Using the values

$$
\begin{aligned}
\frac{d \sigma}{d \Omega} & =44.83 \mu \mathrm{barn} / \mathrm{sr}=44.83 \cdot 10^{-30} \mathrm{~cm}^{2} / \mathrm{sr} \\
I & =100 \mu \mathrm{A}=6.25 \cdot 10^{14} \text { electrons } / \mathrm{s} \\
\rho & =0.0708 \mathrm{~g} / \mathrm{cm}^{3}=4.23 \cdot 10^{22} \text { protons } / \mathrm{cm}^{3} \\
L & =20 \mathrm{~cm}, \\
\Delta \Omega & =2 \times 3.57 \mathrm{msr}, \\
\text { and } f_{b} & =0.8
\end{aligned}
$$

we find that the rate in each spectrometer should be $127.8 \mathrm{MHz}^{\S}$.

The signal in each detector was integrated for $\sim 33 \mathrm{~ms}$ and so an average of 4,217,400 electrons were counted per detector per window, or 8,434,800 per window pair. Since counting statistic determined the minimum width of the statistical distribution of pair asymmetries, we expected a width greater than or equal to

$$
\sigma_{\text {min }}\left(A_{\text {pair }}\right)=\frac{1}{\sqrt{8,434,800}} \approx 344 \mathrm{ppm}
$$

To take full advantage of the available statistics, we required that all other sources of noise in the detector be much less than $344 \mathrm{ppm}$.

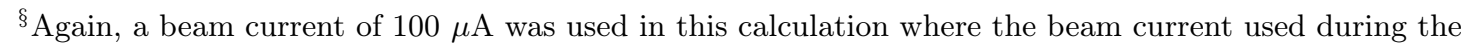
hydrogen run was $35-40 \mu \mathrm{A}$

"Again, this minimum width is for $100 \mu \mathrm{A}$ of current. At the more commonly used beam current of 35 $\mu \mathrm{A}$ for the hydrogen run, $\sigma_{\min }\left(A_{\text {pair }}\right)=582 \mathrm{ppm}$
} 
To estimate the scattering rate for the helium run at a beam energy of $3.4 \mathrm{GeV}$, a $Q^{2}$ of $0.101 \mathrm{GeV}^{2}$, and mean scattering angle of $6^{\circ}$, the following values were used:

$$
\begin{aligned}
\frac{d \sigma}{d \Omega} & =12.5 \mu \mathrm{barn} / \mathrm{sr}=12.5 \cdot 10^{-30} \mathrm{~cm}^{2} / \mathrm{sr} \\
I & =100 \mu \mathrm{A}=6.25 \cdot 10^{14} \text { electrons } / \mathrm{s} \\
\rho & =0.14 \mathrm{~g} / \mathrm{cm}^{3} \\
L & =20 \mathrm{~cm}, \\
\Delta \Omega & =3.7 \mathrm{msr}, \\
\text { and } f_{b} & =0.8 .
\end{aligned}
$$

This gives us a rate in each spectrometer arm of $11.9 \mathrm{MHz} \|$. When the detector signal is integrated over a $\sim 33 \mathrm{~ms}$ window, we expect 392,700 electrons to be counted per window and 785,400 per window pair. Therefore, the minimum detector width for helium was

$$
\sigma_{\min }\left(A_{\text {pair }}\right)=\frac{1}{\sqrt{785,400}} \approx 1130 \mathrm{ppm}^{* *}
$$

Noise in the detector must be kept well below $1130 \mathrm{ppm}$ to take full advantage of the available statistics.

\footnotetext{
" Again, the estimate here assumes a $100 \mu \mathrm{A}$ beam current; it was closer to $30-35 \mu \mathrm{A}$.

**A Again, this minimum width is for $100 \mu \mathrm{A}$ of current. At the more commonly used beam current of 30 $\mu \mathrm{A}$ for the helium run, $\sigma_{\min }\left(A_{\text {pair }}\right)=2060 \mathrm{ppm}$
} 


\subsection{Systematic Error}

Absolute cross-section measurements are very difficult, and it is unrealistic to consider making measurements with part-per-million precision. Asymmetry measurements, however, have several advantages over absolute measurements. The systematic errors in cross-section measurements can be described by three parameters: a scale factor $S$, a common-mode offset $\Delta \sigma_{C M}$, and a helicity-correlated offset $\Delta \sigma_{H C}$, where $\Delta \sigma_{C M}, \Delta \sigma_{H C} \ll \sigma_{R(L)}$. Commonmode offsets (e.g., slow drift in PMT gain or target density) add to the cross-section measurement with the same sign while the helicity-correlated offsets add with the opposite sign. The asymmetry can be written as

$$
A_{\text {meas }}=\frac{S\left(\sigma_{R}+\Delta \sigma_{C M}+\Delta \sigma_{H C}\right)-S\left(\sigma_{L}+\Delta \sigma_{C M}-\Delta \sigma_{H C}\right)}{S\left(\sigma_{R}+\Delta \sigma_{C M}+\Delta \sigma_{H C}\right)+S\left(\sigma_{L}+\Delta \sigma_{C M}-\Delta \sigma_{H C}\right)}
$$

where the scale factor $S$ can be seen to cancel in this ratio, and with a little algebra the expression can be simplified to

$$
A_{\text {meas }} \approx A_{L R}\left(1-\frac{\Delta \sigma_{C M}}{\sigma}\right)+\frac{\Delta \sigma_{H C}}{\sigma}
$$

where $A_{L R}$ is the true asymmetry without any systematic error, $\sigma=\left(\sigma_{R}+\sigma_{L}\right) / 2$ is the average normalized flux, and only first-order terms in $\Delta \sigma_{C M}$ and $\Delta \sigma_{H C}$ have been kept. This equation shows that a fractional error due to a common-mode offset introduces a fractional error of equal magnitude in the true asymmetry. For example, a $1 \%$ error in measuring the normalized flux contributes a $1 \%$ systematic error to the measured asymmetry. Therefore, an asymmetry measurement is fairly insensitive to common-mode offset errors and very in- 
sensitive to scale errors in measuring the normalized flux. The helicity-corrlelated term, however, shows that any systematic error here contributes independent of the true asymmetry, and as a consequence, can be very dangerous. Controlling these helicity-correlated errors at levels well below the proposed statistical errors are necessary to have confidence in the measurement. The details involved in this major effort are described in Chapter 5.

\subsection{Beam Property Asymmetries}

Fluctuations in beam properties such as current, position, angle, and energy on the target can cause fluctuations in the scattering rate into the detector. This is due to the strong dependence of the cross section on scattering angle and energy. Two related methods exist to extract the detector sensitivities to fluctuations in beam properties: using the natural jitter in the beam parameters to study the correlation between detector rate and beam parameters (regression), and deliberately modulating the beam parameters in a controlled way (beam modulation). Both regression and beam modulation analyses assume that for small fluctuations in the beam parameters, the detector's response is linear and can be parameterized as

$$
A_{\text {meas }}=\frac{\Delta D}{2 D}+\frac{\Delta I}{2 I}+\beta_{E} \frac{\Delta E}{2 E}+\Sigma_{i} \beta_{i} \Delta x_{i}
$$

where $D$ is the average detector flux for the right- and left- helicity pulses, $I$ is the beam current, $E$ is the beam energy, the $x_{i}$ run over position and angle in $x$ and $y$, and $\beta_{E}$ and $\beta_{i}$ are the correlation coefficients between energy, position, and angle and the detector signal. These coefficients are measured simultaneously with data-taking during the run. $\Delta$ refers 
to the right-left difference in each of the above parameters.

A regression analysis has two weaknesses that can be overcome by beam modulation. First, correlations between beam parameters make it difficult to extract the contributions to the scattering rate from each individual parameter. This does not mean regression is incorrect; the detector rate is appropriately corrected for fluctuations in beam properties and the raw asymmetry is determined properly. However, it is difficult to determine how much effect each beam parameter had on the detector asymmetry. Beam modulation provides this information by knowingly modulating each beam parameter in a controlled way. By measuring the correlation between the scattering rate and a particular beam parameter while only modulating that parameter, we can measure detector sensitivities due solely to that parameter.

Additionally, it is possible that the natural beam jitter does not sample a region of phase space sampled by helicity correlations in the beam. In this case, the correction for beam helicity correlations would likely be wrong and lead to an unidentified systematic error in the measurement. Beam modulation is designed to span the full phase space and prevent this scenario from happening.

The analysis for HAPPEX-He and $-\mathrm{H}$ relied only on beam modulation. The corrections due to beam fluctuations and helicity correlations were expected to be much smaller than the statistical error, so one method was deemed sufficient. The formalism for beam modulation is presented in Chapter 6 . 


\subsection{Slow Helicity Reversal}

Systematic effects caused by the electronic pickup of the $30 \mathrm{~Hz}$ helicity signal were cancelled by slow, passive reversal of the beam helicity. This reversal was achieved by inserting a half-wave plate into the path of the laser beam just upstream of the Pockels cell. This insertable half-wave plate (IHWP) rotated the incoming linear polarization by $90^{\circ}$ so that circular polarization produced by the Pockels cell with either $R$ or $L$ helicity had its helicity flipped upon IHWP insertion. The key here was that electronic signals in the DAQ were unaware of the switch so that the sign of the measured asymmetry was opposite for the two IHWP states. The sign and magnitude of any electronic pickup of the helicity did not change with the IHWP. This pickup cancelled when measured asymmetries were averaged from the two IHWP states, and this technique minimized false asymmetries and systematic errors associated with helicity-correlated pickup. The IHWP was inserted and removed from the beamline every few hours of data-taking, hence the label, "slow helicity reversal".

\subsection{Backgrounds}

Using the integration technique for data-taking presents the challenge of how to measure various background signals which cannot be separated from the data. At the detector location, the Hall A High Resolution Spectrometer (HRS) does provide a very clean separation of the data from the background, and various dedicated runs were used to measure that which was not cleanly separated from the detected flux. Corrections to the raw asymmetry 
can be made according to

$$
A_{L R}=\frac{A_{\mathrm{raw}} / P_{B}-f_{\mathrm{bkg}} A_{\mathrm{bkg}}}{1-f_{\mathrm{bkg}}}
$$

where $f_{\mathrm{bkg}}$ and $A_{\mathrm{bkg}}$ are the fraction of background found measured in the detector flux and the parity-violating asymmetries coming from that background source. Sources of background contamination during the experiment run included quasielastic scattering from the ${ }^{4}$ He nucleus, quasielastic scattering from the end-windows of the aluminum target, rescattering of the electron, and scattering from the poletip of the spectromter magnet. All of these sources have parity-violating asymmetries capable of biasing the physics asymmetry if contamination in the detector flux is significant. Many of the asymmetries were estimated from calculations, and corrections were made when necessary. All background fractions were kept small so as to keep the systematic error on the measured asymmetry small.

\subsection{Polarimetry}

The parity-violating asymmetry is proportional to the beam polarization $P_{B}$, so the polarization must be carefully measured and monitored throughout the experiment's run. We can show this by imagining the cross section as having two components: a parity-conserving piece $\sigma_{E M}$ due the electromagnetic interaction and a parity-violating piece $\sigma_{P V}$ due to the interference of the weak and electromagnetic scattering amplitudes. The parity-violating piece scales with $P_{B}$ and $\sigma_{P V}$ adds to $\sigma_{E M}$ with opposite signs for each helicity state. We 
can write $\sigma_{R}=\sigma_{E M}+\sigma_{P V}$ and $\sigma_{L}=\sigma_{E M}-\sigma_{P V}$, and the asymmetry as follows:

$$
A_{\text {raw }}=\frac{\left(\sigma_{E M}+P_{B} \sigma_{P V}\right)-\left(\sigma_{E M}-P_{B} \sigma_{P V}\right)}{\left(\sigma_{E M}+P_{B} \sigma_{P V}\right)+\left(\sigma_{E M}-P_{B} \sigma_{P V}\right)} \approx P_{B} \frac{\sigma_{P V}}{\sigma_{E M}}=P_{B} A_{P V}
$$

Since $A_{P V}$ scales with $P_{B}$, the systematic error in the asymmetry is just the fractional error in the beam polarization. This is one of the dominant errors in the asymmetry, and it must be monitored closely. There were three independent polarization measurements used for HAPPEX: a Mott polarimeter in the injector of the accelerator and the Moller and Compton Polarimeters in Hall A (all discussed further in Chapter 4). The Mott polarimeter was used by the Injector group to characterize the polarization as a function of position across the cathode and select spot providing high polarization. The Moller Polarimeter required auxiliary runs to make measurements while the Compton Polarimeter could be used to continuously monitor the beam polarization during data taking. All were believed to provide measurements of beam polarization with a relative error of better than $3 \%$. 


\section{Chapter 4}

\section{Experimental Apparatus}

\subsection{Accelerator at TJNAF}

The Thomas Jefferson National Accelerator Facility (TJNAF) in Newport News, VA, is a United States Department of Energy facility designed to investigate the structure of nuclei and hadrons. This facility operates a radio frequency $(\mathrm{RF})$ electron accelerator capable of re-circulating the beam five times through two superconducting linear accelerators (linac). The beam is sent to three experimental halls (Hall A, B, and C). Hall C has been operational since November 1995, Hall A since May 1997 and Hall B since December 1997.

The accelerator uses a photocathode gun system capable of delivering up to $200 \mu \mathrm{A}$ of continuous-wave $(\mathrm{CW})$ electron beam at high polarization $(>80 \%)$ which can be split arbitrarily between three interleaved $499 \mathrm{MHz}$ bunch trains. One such bunch train can be peeled off after each linac pass to one of three experimental halls using RF separators and septa [61] (labeled extraction elements in Figure 4.1). Polarized electrons from the source in the injector are first accelerated to $45 \mathrm{MeV}$, then are injected into the north linac. Twenty 
RF cryomodules, each containing eight superconducting niobium cavities, comprise the two linacs. Liquid helium, produced at the Central Liquid Helium Liquefier (CHL), keeps the accelerating cavities superconducting at a temperature of $2 \mathrm{~K}$. The linac energies are each set identically and the RF cavities are phased to provide maximum acceleration. Quadropole and dipole magnets in each arc provide the field which focuses and steers the beam as it passes through each arc. The nominal gain of each linac was designed to be about $400 \mathrm{MeV}$, however, this gain can be tuned up to $500 \mathrm{MeV}$, if required by the experimental halls. This has made it possible to accelerate electrons up to $5.9 \mathrm{GeV}$.

The 2005 HAPPEX measurements were carried out in Hall A with a 35 to $55 \mu \mathrm{A}$ beam of $\sim 3 \mathrm{GeV}$ longitudinally polarized electrons incident on a $20 \mathrm{~cm}$ long cryogenic target. The data collecting took place over 55 days $\left({ }^{4} \mathrm{He}\right)$ and 36 days $\left({ }^{1} \mathrm{H}\right)$ over July to November. After scattering from the cryogenic target, the electrons entered the High Resolution Spectrometers (HRS) where they were focused onto the HAPPEX detectors, and various Hall A beamline components monitored the beam polarization, current, position and energy. The remainder of this chapter will discuss the experimental apparatus used to make this parity-violating asymmetry measurement.

\subsection{Polarized Source}

The HAPPEX collaboration has devoted great time and effort into understanding the polarized electron source. Careful setup of the polarized source optics is important for minimizing helicity-correlated beam asymmetries ( ${ }^{\text {beam }} \mathrm{A}_{\mathrm{LR}}$ 's), an important factor in the experiment's systematic error. All important components of JLab's polarized source will be described in 


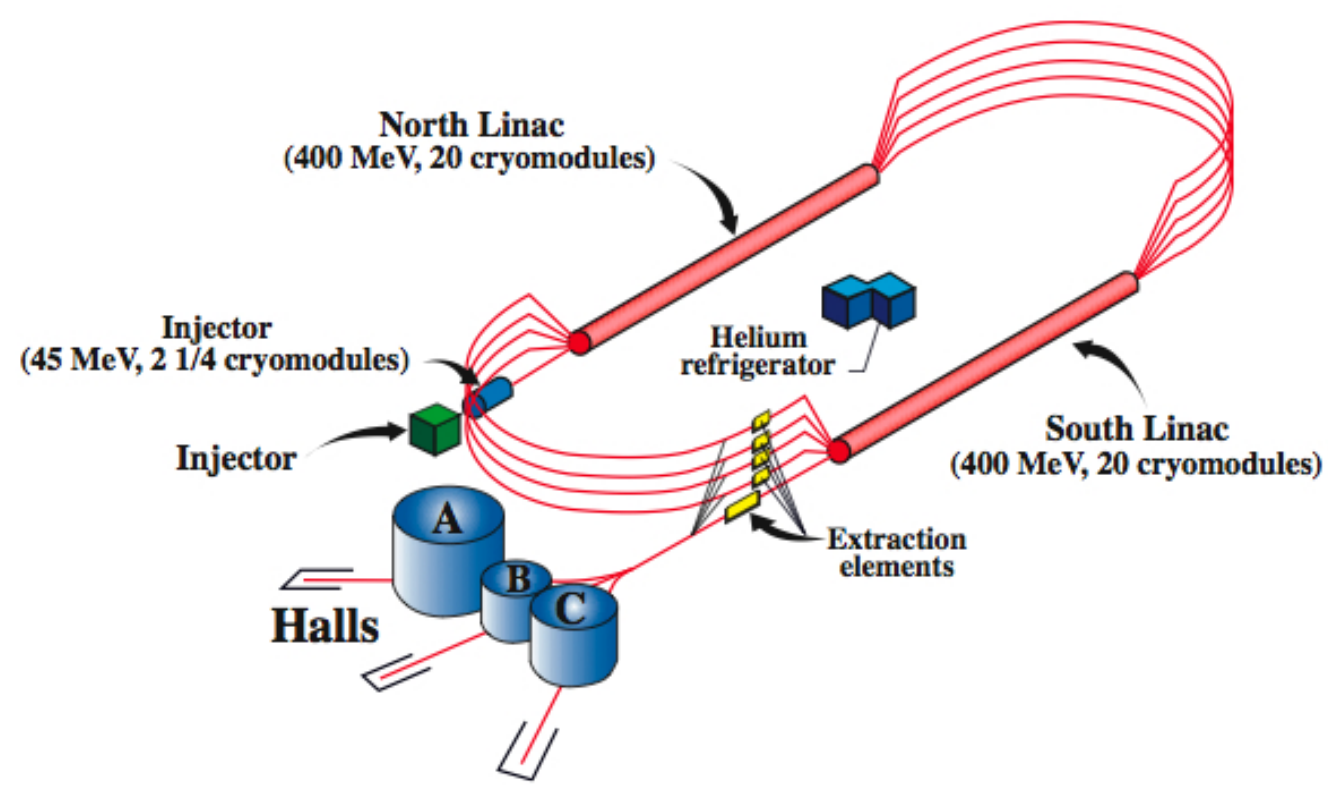

Figure 4.1: Schematic of TJNAF accelerator and the experimental halls

this section, and the sources of and methods for reducing the important helicity-correlated beam asymmetries will be discussed in Chapter 5 .

\subsubsection{GaAs Photocathode}

Circularly polarized laser light produces longitudinally polarized electrons through photoemission from a superlattice Gallium-Arsenide (GaAs) photocathode. During HAPPEX, the cathode was illuminated by a Tiger-CD Ti:Sapphire laser manufactured by Time-Bandwidth Products in Zurich, Switzerland, and tuned to $800 \mathrm{~nm}$. After laser light excites an electron from the valence to the conduction band, the prepared negative electron affinity of the crystal's surface allows the electron to move to the surface of the crystal and escape into the vacuum. A $-100 \mathrm{keV}$ bias voltage at the GaAs crystal allows it to act as a cathode, and electrons are drawn away from the crystal and into the accelerator.

The cathode consists of several layers containing GaAs with varying amounts of phospho- 


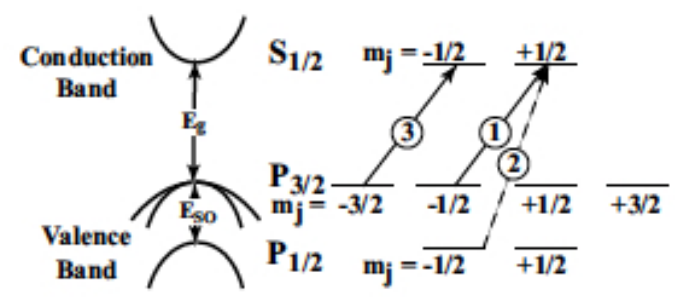

(a) Bulk or unstrained GaAs.

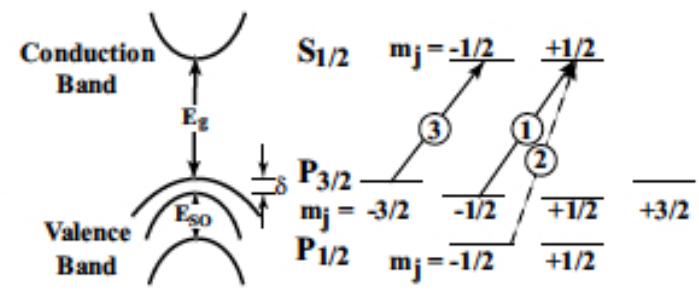

(b) Strained GaAs.

Figure 4.2: Band Gap Structure of GaAs. The relative transmission probabilities for incident righthelicity light are indicated by the circled numbers. $E_{g}$ is the band gap energy, typically $\sim 1.6 \mathrm{eV}$. $E_{S O}$ is the splitting of the valence band $P_{1 / 2}$ and $P_{3 / 2}$ levels due to the spin-orbit interaction. In (b), $\delta$ is the energy gap $(\sim 50 \mathrm{meV})$ due to the broken degeneracy of the $P_{3 / 2}$ states from the strain applied by the mismatches in lattice constants.

rus doping, grown on substrate [47]. The conduction band is the $S_{1 / 2}$ band and the valence band is split into two bands, $P_{1 / 2}$ and $P_{3 / 2}$, by the spin-orbit interaction with phosphorous. The $P_{1 / 2}$ band is neglected for our purposes. In a "bulk" GaAs crystal, the $P_{3 / 2}$ level is a fourfold degenerate making the maximum achievable polarization only $50 \%$. When a strain is applied to the crystal, this degeneracy is broken making the theoretically maximum polarization $100 \%$ with proper tuning of the laser light's wavelength. A strain to the crystal is added by growing a $\sim 100 \mathrm{~nm}$ layer of GaAs on a substrate of GaAsP; the mismatch in lattice constants between the two layers induces the required strain. A "bulk" cathode was used during the 1998 HAPEX run and achieved a beam polarization of 38\%. A strained layer was used in 1999 and provided one of $71 \%$ [74]. The band structures of both the "bulk" and strained GaAs cathodes are displayed in Figure 4.2.

In a strained GaAs crystal, the quantum efficiency (QE) for incident linearly polarized light depends on the polarization's orientation with respect to the axis aligned with the "strain". This is referred to as a "QE anisotropy", and has been observed to depend on a cathode's structure [50]. This anisotropy is defined as $\frac{\Delta Q E}{2\langle Q E\rangle}$ where $\Delta Q E$ is the difference 


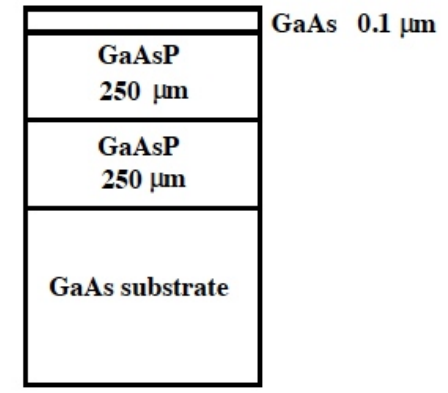

(a) Strained-layer Photocathode

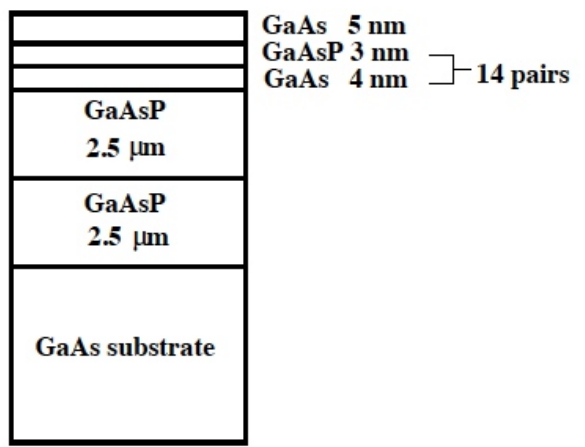

(b) Superlattice Photocathode

Figure 4.3: Structure of the (a) strained-layer photocathode and the (b) superlattice photocathode between the maximum and minimum QE's and $\langle Q E\rangle$ is their average.

As the strained photocathode allowed for a higher beam polarization, even higher polarizations were observed with a superlattice cathode used for the HAPPEX 2005 run. In 1999, there were limits to the polarization due to a relaxation of the strained layer below a certain critical layer thickness, about 10nm. Layers in the superlattice were much smaller than in the original strained layer, and it was believed to lessen this depolarization effect [48]. The structures of both the strained-layer and superlattice cathode are shown in Figure 4.3. The quantum efficiency $(\mathrm{QE})$ was as high as $1 \%$ for the superlattice cathode compared to only $0.2 \%$ for the strained-layer cathode. The QE anisotropy differed between the cathodes with typical values of about $12 \%$ for the strained-layer and only $4 \%$ for the superlattice [51].

The QE anisotropy can generate helicity-correlated beam asymmetries on the electron beam when the laser light illuminating the GaAs crystal is not perfectly circularly polarized. In practice, perfect circular polarization cannot be attained, and some residual linear polarization will remain. This linear polarization will generally differ for the laser light's two 
helicity states, and the QE seen by the two states will differ as a consequence. An asymmetry in the number of electrons pulled from the cathode will result. This coupling between the QE anisotropy and residual linear polarization is a dominant source of beam ${ }^{2}{ }_{\mathrm{LR}}$ 's, and is discussed in detail in Chapter 5.

\subsubsection{Source Optics}

A diagram of the source laser table used in the accelerator tunnel is displayed in Figure 4.4. There are two identical photocathode guns in the JLab injector referred to as Gun \#2 and Gun \#3. Both guns had a superlattice GaAs cathode installed in them for the experiment run, and these guns are used to eject electrons into the accelerator. The laser light travelled to cathode Gun \#3 (from an outrigger table not shown in Figure 4.4) for the ${ }^{4}$ He run and Gun \#2 for the ${ }^{1} \mathrm{H}$ run. The laser beam trajectory for each cathode gun is pretty similar with only some additional elements needed for the Hall A line due to the strict systematic requirements. All halls have attenuators to adjust the laser power as the QE of the cathode declines; Halls A and C had "tune-mode" optics that produced a pulsed structure to the beam making it easier to steer and avoid beamline elements; Hall B lacks these optics as it operates at so low a current to not need them.

\section{Hall A Line}

The Hall A line includes a beam telescope that is used to vary the beam spot size and angle of divergence at the Pockels cell $(\sim 1 \mathrm{~mm})$ and cathode. It consists of two tiny lenses each with focal lengths of $6.5 \mathrm{~mm}$ placed just centimeters apart. An IA system consisting of a Pockels cell (see next section) and a linear polarizer is shown in the Hall A line in Figure 


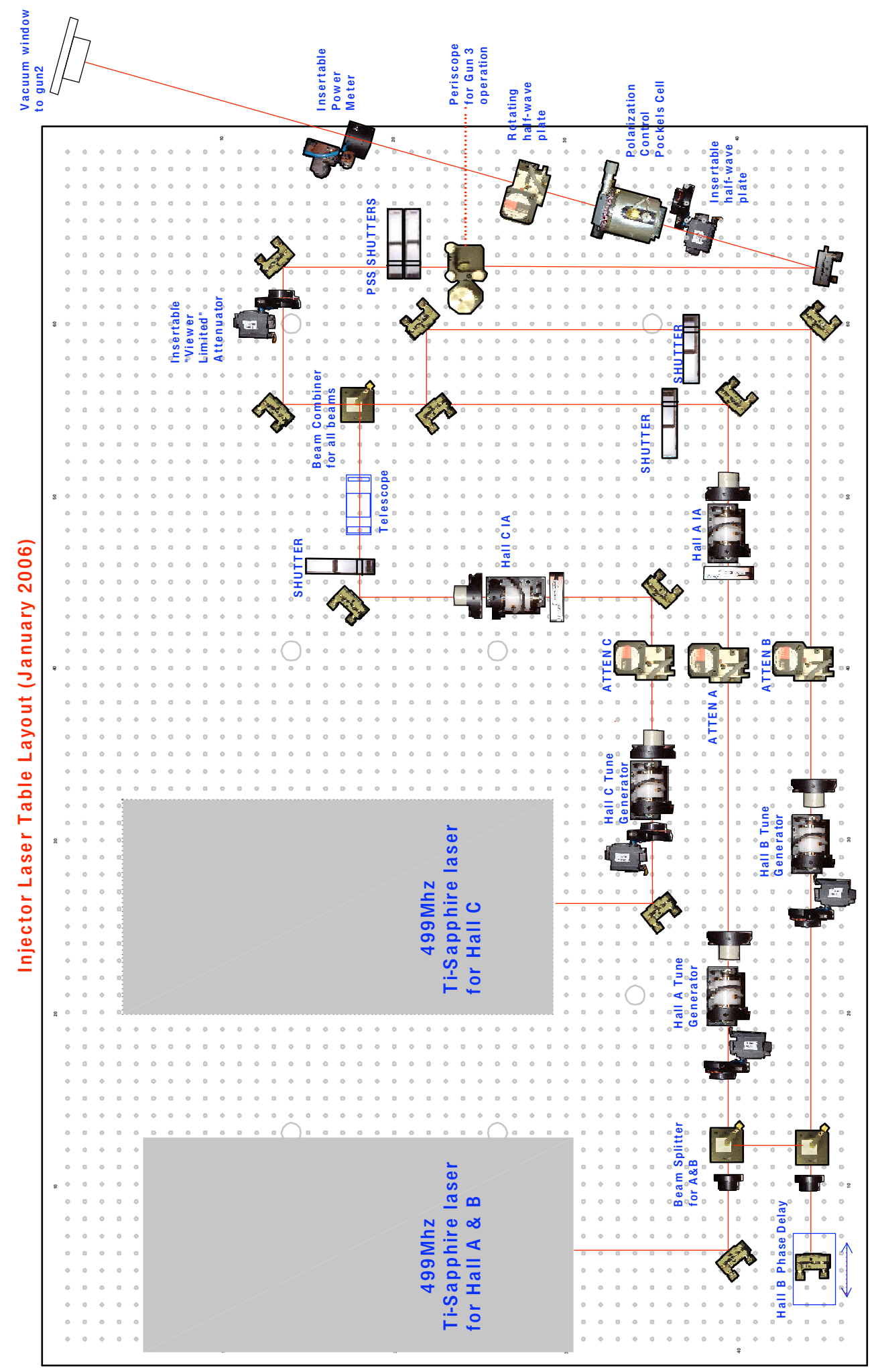

Figure 4.4: The JLab polarized source laser system as it was configured for the HAPPEX run. The outrigger table providing the line to cathode Gun \#3 is not shown here, but was located to the right of the optics table. 
4.4 (labelled "Hall A IA"). The IA system was typically used for asymmetry feedback, but only the Pockels cell (labelled "Polarization Control Pockels Cell" in Figure 4.4) was used for feedback in 2005. However, Hall C had an IA system (labelled "Hall C IA" in Figure 4.4) used to feedback on its own charge asymmetry $A_{Q}$ and minimize effects in Hall A due to the interactions of Hall C's beam with the cathode. Asymmetry feedback is discussed in Section 5.4.2.

\section{Pockels Cell}

The Pockels Cell (PC) is crucially involved in a parity-violating experiment because it provides the fast reversal of the electron beam's helicity. The PC is an electro-optic device whose birefringence is proportional to the voltage applied to it; it functions as a quarter-wave plate converting linear into circularly polarized laser light. The PC used in this experiment was made by Cleveland Crystals of the material $\mathrm{KD}^{*} \mathrm{P}\left(\mathrm{KD}_{2} \mathrm{PO}_{4}\right)$. Voltages of between $\sim \pm 2300$ and $\sim \pm 2500 \mathrm{~V}$ are applied for laser light wavelengths of $\sim 800 \mathrm{~nm}$ to achieve a birefringence capable of producing a quarter-wave retardation between the fast and slow axes of the PC. The sign of the voltages applied to the PC were flipped at a rate of $30 \mathrm{~Hz}$ to reverse the helicity of the outgoing laser light. An insertable half-wave plate upstream of the Pockels cell rotated the incoming linear polarization incident on the PC by $90^{\circ}$ to reverse the beam's outgoing helicity with respect to the electronic helicity signal. This is done to allow for some cancellation of helicity-correlated beam asymmetries not dependent on laser beam polarization (e.g., steering).

A well-aligned Pockels cell will still produce circularly polarized laser light with some imperfections. Any residual linear polarization in the laser light will likely create charge 
asymmetries and position differences when analyzed by the cathode. A rotating half-wave plate (RHWP) was placed downstream of the Pockels cell to rotate the residual linear polarization in an attempt to minimize such helicity-correlated beam asymmetries. These asymmetries will be examined in further detail in Chapter 5.

\section{Transport to Cathode and Injector}

After some days of the laser light hitting the cathode, a QE hole can develop making photoemission less efficient, thus lessening the amount of beam current delivered into the hall. QE holes can also contribute to the existence of helicity-corrleated beam asymmetries. A lens was placed upstream of the vacuum window entrance in order to steer the laser light onto a new position on the cathode when necessary.

There were two separate paths for the laser light on the laser table. A periscope was used to direct the light to a separate outrigger table to steer the beam into Gun $\# 3$. There was a pick-off beamline which threw the light a distance equal to that remaining to the cathode into a CCD camera. This allowed a measurement of the laser beam's spot size.

Each cathode sat at an angle of $15^{\circ}$ with respect to the accelerator beamline. A Wien filter was used to set the angle of the electron's polarization upon entering the accelerator; the angle was set so as to make the electron's polarization longitudinal in the experimental hall after considering the electron's $g-2$ precession through the entire path of the accelerator. Electrons are accelerated up to $45 \mathrm{MeV}$ before entering the main accelerator. 


\subsection{Beamline}

The Hall A beamline starts after the arc section (used for beam energy measurement) and ends at a beam dump. The arc section can be use for the beam energy measurement, and is described in section 4.3.1. The beamline included a Compton beam polarimeter, two Beam Current Monitors (BCM) between which is located an "Unser" monitor (for absolute beam current measurement), a fast raster, an eP device for beam energy measurement, a Møller beam polarimeter, and a number of Beam Position Monitors (BPM). The details of these elements are described in the following sections.

\subsubsection{Beam Energy Measurement}

The energy of the beam is measured absolutely by two independent methods [53]: the Arc method in which the beam deflection is measured in the arc section of the beamline, and the eP method which was based on elastic electron-proton scattering. Both methods provided a precision of $\delta E_{\text {beam }} / E_{\text {beam }} \sim 2 \times 10^{-4}$.

\section{Arc measurement}

The Arc measurement was based on the physical principle that an electron in a magnetic field moves in a circle, the radius of which depends on the field's magnitude and the electron's momentum. The Arc method measured the deflection of the beam in the arc section of the beamline, and was performed when the beam was tuned in "dispersive mode" in the arc section. A detailed description of the instrumentation used in the Arc measurement can be found in [54]. The nominal bend angle in the arc section was $\phi=34.3^{\circ}$, see Figure 4.5. The 


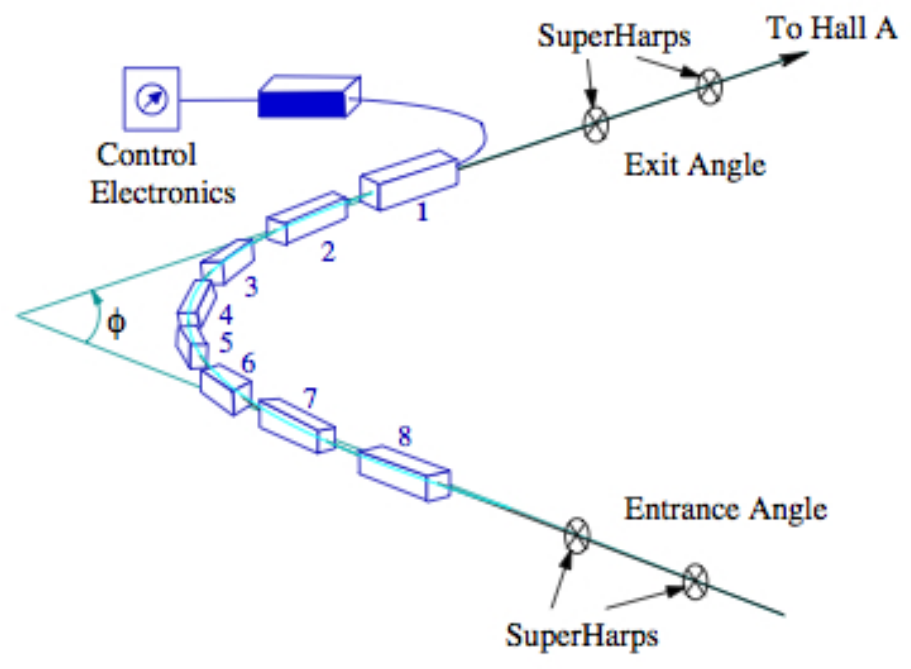

Figure 4.5: The arc section of the beamline

momentum of the electron beam $(p$ in $\mathrm{GeV} / c)$ is related to the field integral of the eight dipoles $\left(\int \vec{B} \cdot \overrightarrow{d l}\right.$ in Tm) and the net bend angle through the arc section ( $\theta$ in radians) by

$$
p=k \frac{\int \vec{B} \cdot \overrightarrow{d l}}{\theta}
$$

where $k=0.299792 \mathrm{GeV} \operatorname{rad} \mathrm{T}^{-1} \mathrm{~m}^{-1} / c$. This method consists of two simultaneous measurements, one for the field integral of the bending elements (eight dipoles in the arc), based on a reference magnet (9th dipole) measurement, and the actual bend angle of the arc, based on a set of wire scanners (SuperHarps). As a SuperHarp was moved across the beam path, particles scattering off the wire were collected by a simple ion chamber generating a current and recording the beam's position [55]. 


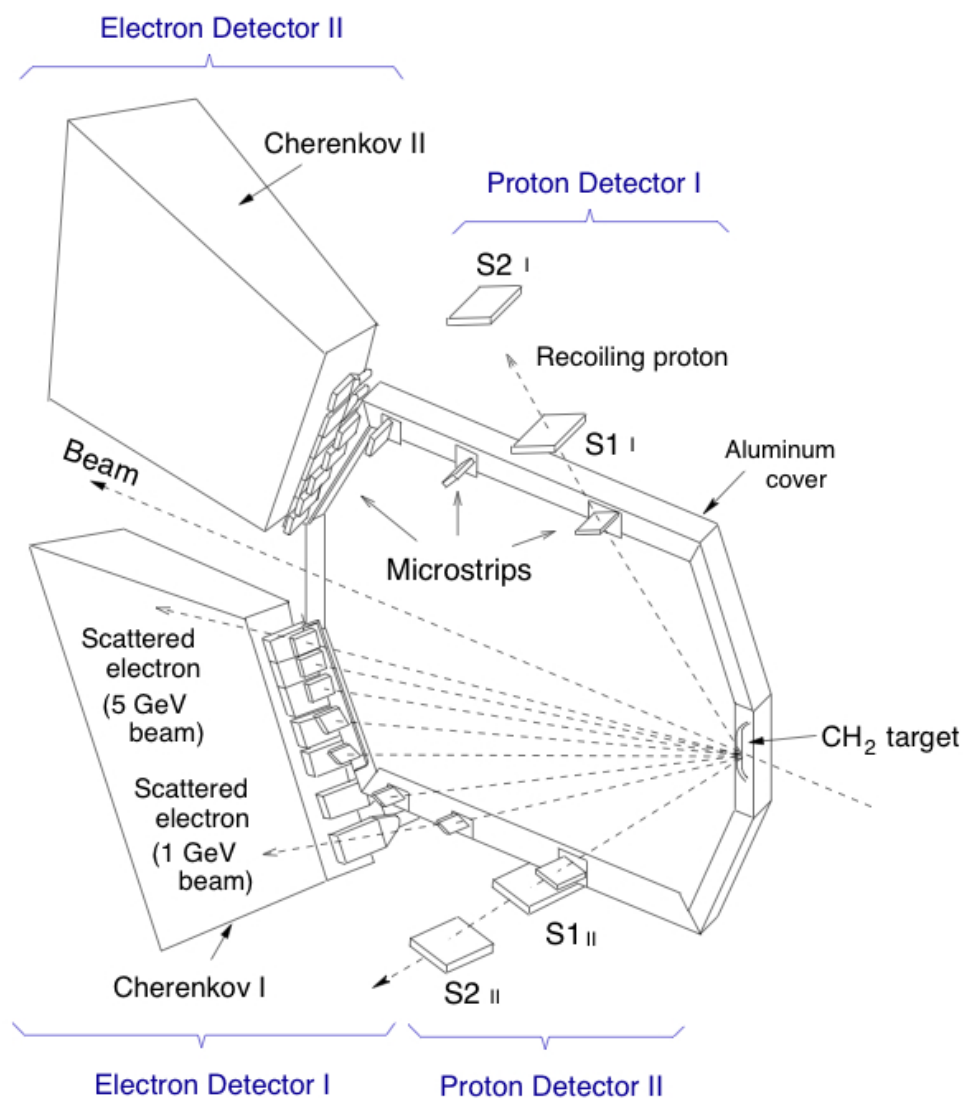

Figure 4.6: Layout of the eP device. 


\section{eP measurement}

The eP measurement was made with a stand-alone device along the beamline located $17 \mathrm{~m}$ upstream of the target. This device measured the scatterd electron angle $\theta_{e}$ and the angle of the recoiled proton $\theta_{p}$ in the ${ }^{1} \mathrm{H}\left(\mathrm{e}, \mathrm{e}^{\prime} \mathrm{p}\right)$ elastic reaction according to the kinematic formula

$$
E=M_{p} \frac{\cos \left(\theta_{e}\right)+\sin \left(\theta_{e}\right) / \tan \left(\theta_{p}\right)-1}{1-\cos \left(\theta_{p}\right)}+O\left(m_{e}^{2} / E^{2}\right)
$$

where $M_{p}$ is the mass of the proton and $m_{e}$ the mass of the electron.

A three-dimensional schematic diagram of the eP device is shown in Figure 4.6. A detailed description of the eP system's instrumentation can be found in [54]. The target was a polyethylene film $\left(\mathrm{CH}_{2}\right)$ enclosed in aluminum. Two identical arms, each consisting of an electron and proton detector system, were symmetrically places with respect to the beam in the vertical plane. Each proton detector was composed of a set of $2 \times 8$ silicon micro-strip detectors. The trajectories of the scattered electrons and recoil protons depend on beam energy, as well as its position and direction. Simultaneous measurements of the scattering in both arms result in first order cancellation of uncertainties in the knowledge of the position and direction of the beam.

Even though both the eP and Arc measurements have shown good agreement with each other within their respective uncertainties after repeated measurements (discrepancy $\leq 3 \times 10^{-4}$ ), their measurements do differ around a beam energy of $3 \mathrm{GeV}$. As different microstrip detectors are used for different energy ranges, it is conjectured that the discrepancy at $3 \mathrm{GeV}$ is due to the misalignment of a particular microstrip in the eP setup [61]. 


\section{Beam Energy During Happex-He and -H}

Table 4.1 shows the beam energy measurements made during Happex-He.

\begin{tabular}{c|c|c|c|c}
\hline \hline Date & Arc & eP & Tiefenbach* $^{*}$ & Set Energy \\
\hline $08 / 16 / 2005$ & $2756.8 \pm 0.65 \pm 0.6$ & & $2753.3 \pm 3$ & 2750.63 \\
$09 / 15 / 2005$ & & $2749.27 \pm 0.288 \pm 0.57$ & $2753.3 \pm 3$ & 2750.63 \\
\hline \hline
\end{tabular}

Table 4.1: Summary of Beam Energy Measurements for ${ }^{4} \mathrm{He}$. All energies are given in $\mathrm{MeV}$.

Neither an Arc or eP measurement was taken for the ${ }^{1} \mathrm{H}$ measurement. The variation of the Tiefenbach energy was only about $\pm 0.4 \mathrm{MeV}$ over the course of the ${ }^{4} \mathrm{He}$ run, and the decision was made to use it during ${ }^{1} \mathrm{H}$. The Tiefenbach energy measured during ${ }^{1} \mathrm{H}$ was $3176 \pm 3 \mathrm{MeV}$.

\subsubsection{Current Monitors}

Hall A's beam current monitor (BCM) was designed to provide a stable, low-noise, noninterfering measurement [61]. It consisted of an "Unser" monitor and two RF cavities located $\sim 25 \mathrm{~m}$ upstream of the target in a temperature-stabilized box with the cavities ("BCM1" and "BCM2") symmetrically placed upstream and downstream of the Unser. The "Unser" monitor [56] is a Parametric Current Transformer that provides an absolute measure of the beam current. It was calibrated by passing a known current through a wire inside the beam pipe and had a nominal output of $4 \mathrm{mV} / \mu \mathrm{A}$. It required extensive magnetic shielding and temperature stabilization to reduce noise and zero drift [61]. It was unreliable as a continuous current monitor, as its output was unstable over the period of a few minutes.

\footnotetext{
*The "Tiefenbach" energy uses the current values of Hall A arc Bdl and Hall A arc beam position monitors (BPMs) to calculate the beam energy. This number is continuosly record in the data stream and is regularly calibrated against the 9 th dipole Arc energy.

†The "Set Energy" is the beam energy at which the accelerator is set to run. This is based on the field maps of the recirculation magnets and the Hall's energy measurements.
} 


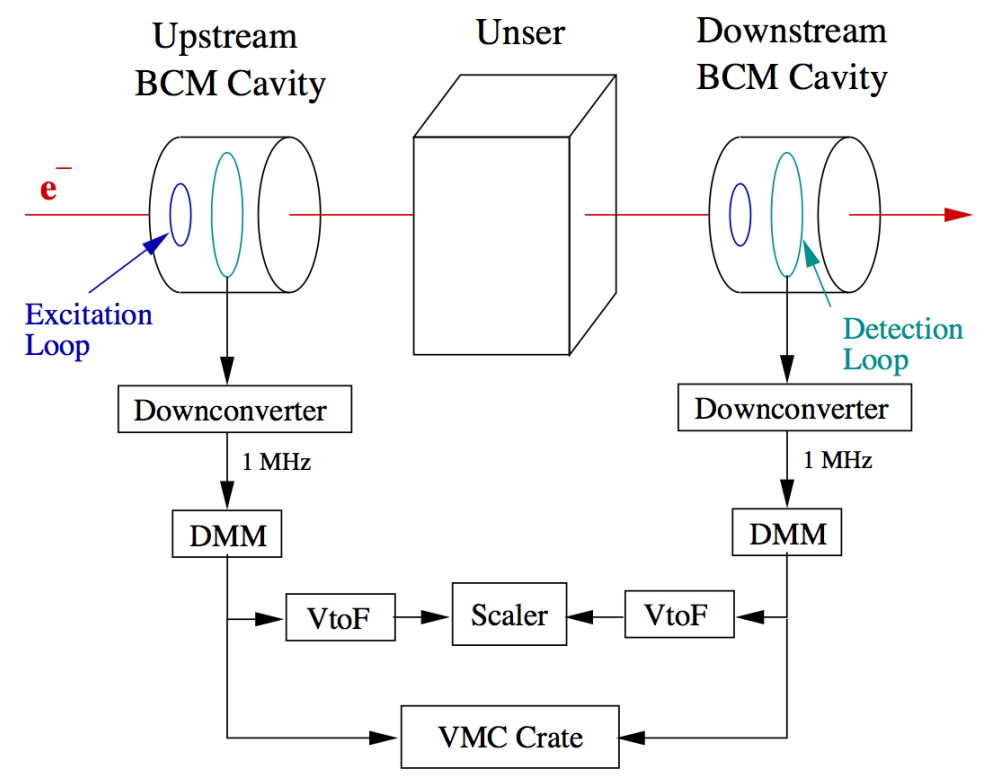

Figure 4.7: Schematic of the beam current monitors.

The "Unser" was used instead for calibration of the RF cavities. A schematic of these beam current monitors is displayed in Figure 4.7.

The two resonant RF cavities were stainless steel cylindrical high Q $(\sim 3000)$ waveguides tuned to the frequency of the beam $(1.497 \mathrm{GHz})$. This allowed the voltage levels at their outputs to be proportional to the beam current. Each of the output signals from the two cavities were split into two parts: to be sampled or integrated. The sampled data was sent to a high-precision digital $\mathrm{AC}$ voltmeter, a device that provided, once every second, a digital output representing the RMS of the input signal during that second. The data to be integrated was sent to a RMS-DC converter which produced a DC voltage level. This level drove a Voltage-to-Frequency converter whose frequency output was proportional to the DC input. This data was accumulated during the run resulting in a number proportional to the time-interated voltage level, a more accurate representation of the total beam charge. 
For BCM2, the HAPPEX DAQ read out three different amplifications: 1x, 3x, and 10x. The amplifications are designated "BCM2", "BCM3", and "BCM4", respectively, in the data stream. The $1 \mathrm{x}$ signals are linearly responsive above $\sim 10 \mu \mathrm{A}$ and from $\sim 2 \mu \mathrm{A}$ for the amplified signals.

\subsubsection{Beam Position Monitors}

The beam position was measured at several points along the $100 \mathrm{keV}$ and $5 \mathrm{MeV}$ regions of the injector and at five points along the beamline leading to Hall A. Beam position monitors (BPM's) at these locations were composed of four wire antennas arranged parallel to the beam direction as shown in Figure $4.8[57,58]$; the antenna were arranged in a square rotated $45^{\circ}$ from the horizontal (the monitors at $100 \mathrm{keV}$ were an exception to this).

Each wire received an RF signal from the beam that when processed yielded a DC signal proportional to the beam current times the distance between the wire and the beam. The signal from opposing wires was multiplexed through the same electronics channel, and a gain switch was used to keep the DC signal approximately constant regardless of beam current. This technique helped to maintain similar pedestals and gains between the wires. A difference over the sum calculation was used to calculate the beam position ( $X^{\prime}$ and $Y^{\prime}$ ) along the axis of the wires:

$$
X^{\prime}=\frac{\kappa}{2}\left(\frac{X_{+}-X_{-}}{X_{+}+X_{-}}\right), \quad Y^{\prime}=\frac{\kappa}{2}\left(\frac{Y_{+}-Y_{-}}{Y_{+}+Y_{-}}\right)
$$

where $\kappa=37.52 \mathrm{~m}$ and is the distance between the wires. All BPM's beyond the $5 \mathrm{MeV}$ 

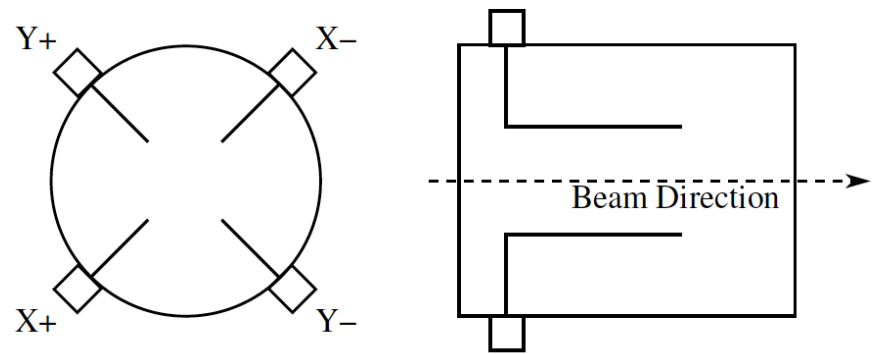

Figure 4.8: Schematic of a stripline beam position monitor. The view along the beam axis is shown on the left while a side view of the monitor is shown on the right.

region of the injector require a $45^{\circ}$ rotation to reflect their true lab orientation:

$$
\left(\begin{array}{c}
X \\
Y
\end{array}\right)=\left(\begin{array}{cc}
\cos \left(45^{\circ}\right) & -\sin \left(45^{\circ}\right) \\
\sin \left(45^{\circ}\right) & \cos \left(45^{\circ}\right)
\end{array}\right)\left(\begin{array}{c}
X^{\prime} \\
Y^{\prime}
\end{array}\right)
$$

\subsubsection{Modulation Coils}

The electron-proton and electron-helium scattering elastic cross-sections are dependent upon the incident electron's scattering angle and energy; therefore, small changes in these quantities will change the cross-section and if these changes are helicity-dependent, will create a false asymmetry. A measurement of the sensitivity of the detected scattering rate to changes in these parameters is necessary so that corrections to the physics asymmetry could be made.

A technique called beam modulation was used to measure the cross-section's sensitivity; 7 magnetic coils located several meters upstream of Hall A's main bend in its beamline and an energy vernier of a cryo-module in the accelerator's South Linac were used in the procedure. Every 10 minutes during a production run, each modulation coil was ramped up and down in current to move or "dither" the electron beam by some known amount; the last cycle of this procedure was modulation of the energy vernier. Responses of the beam 


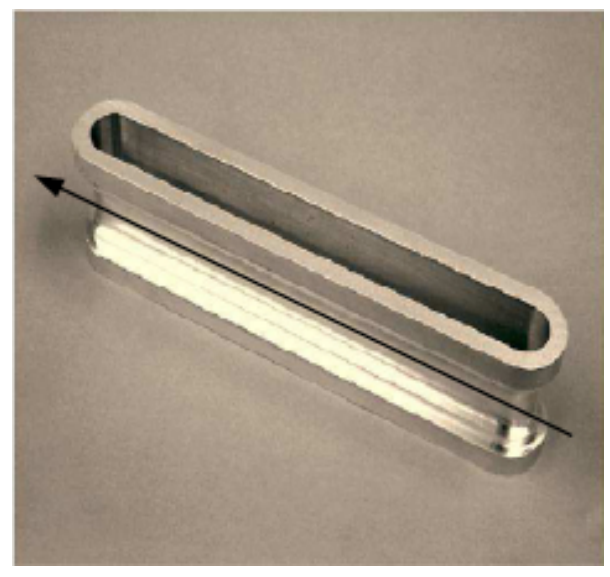

Figure 4.9: Picture of the racetrack target cell

monitors and detectors were measured and the data were used to determine the detector response to changes in position and energy (see section 6.1.2, Beam Modulation Analysis).

\subsection{Target and Raster}

Approximately $0.8 \mathrm{~m}$ upstream of the spectrometers' pivot (the point about which each spectrometer's azimuthal position is determined) sat a $1.2 \mathrm{~m}$ diameter scattering chamber maintained at $10^{-6}$ torr that housed the cryogenic targets for both the ${ }^{4} \mathrm{He}$ and ${ }^{1} \mathrm{H}$ runs. Scattered electrons from the targets passed through a transfer box which housed a pair of tungsten acceptance defining apertures which limited the amount of direct heating to the septum coils due to scattered electrons from outside the septum's acceptance. The spectrometer and septum are described in Section 4.5.

The target ladder was supported from the top of the scattering chamber, was made of aluminum, and was controlled remotely from the counting house. The target cells at the top of the ladder were coupled to one of the cryogenic loops.

For both ${ }^{4} \mathrm{He}$ and ${ }^{1} \mathrm{H}$, the production target cell was a specially designed [59] $20 \mathrm{~cm}$ 


\begin{tabular}{c|r|r}
\hline \hline Target & Thickness (mm) & Length $(\mathrm{mm})$ \\
\hline $20 \mathrm{~cm}^{4} \mathrm{He}$ & (entr) $0.178 \pm 0.02$ & $19.81 \pm 0.02$ \\
& (exit) $0.213 \pm 0.02$ & \\
& (side) $0.290 \pm 0.02$ & \\
\hline $20 \mathrm{~cm} \mathrm{LH}_{2}$ & (entr) $0.178 \pm 0.02$ & $19.95 \pm 0.02$ \\
& (exit) $0.071 \pm 0.02$ & \\
\hline Carbon Foils & $0.173 \pm 0.001$ & $\pm 12 \mathrm{~cm}$ \\
\hline Aluminum Foils & $1.00 \pm 0.02$ & $\pm 10 \mathrm{~cm}$ \\
\hline Single Carbon Foil & $0.173 \pm 0.001$ & \\
\hline BeO & \multicolumn{1}{|}{} \\
\hline \hline
\end{tabular}

Table 4.2: Dimensions of targets used in the experiment

racetrack cell which featured a cryogenic flow transverse to the beam direction. A picture of the racetrack cell is displayed in Figure 4.9. The previously used 15 and $4 \mathrm{~cm}$ "beercan" cells (provided by Coors Brewing Company, Golden, CO) were included on the ladder as a backup, and a variety of solid target foils were included below them. The cryogenic loop that supplied the target gas was filled with ${ }^{1} \mathrm{H}$ or ${ }^{4} \mathrm{He}$ at room temperture; the gas was cooled through a heat exchanger supplied with $4 \mathrm{~K}{ }^{4} \mathrm{He}$ from the Central Helium Liquefier (CHL). A fan was used to regulate target cryogen flow to maintain a stable temperature, and a heater was used to maintain the temperature when the beam is off.

The dimensions for the various targets on the target ladder, including the two racetracks for ${ }^{4} \mathrm{He}$ and ${ }^{1} \mathrm{H}$, are displayed in Table 4.1. The liquid hydrogen $\left(\mathrm{LH}_{2}\right)$ cryotarget was operated at a temperature of $19 \mathrm{~K}$ and pressure of $\sim 25$ psia with a density of $0.072 \mathrm{~g} / \mathrm{cm}^{3}$. The operating parameters for the ${ }^{4} \mathrm{He}$ cryotarget are listed in Table 4.2.

The power deposited on the target by the electron beam can lead to fluctuations in the target density. If significant, this results in a decrease in the effective target length leading to a drop in the detected rate of scattered electrons; more importantly is the possibility that 


\begin{tabular}{lc}
\hline \hline Parameter & Value \\
\hline Temperature & $6.6(7.0) \mathrm{K}$ \\
Pressure & $175(195) \mathrm{psi}$ \\
Density & $0.128 \mathrm{~g} / \mathrm{cm}^{3}$ \\
Fan Speed & $48 \mathrm{~Hz}$ \\
CHL 4 K Flow & $17(4) \mathrm{g} / \mathrm{s}$ \\
Typical Total Power Deposition & $\sim 205 \mathrm{~W}$ \\
\hline \hline
\end{tabular}

Table 4.3: Parameters for ${ }^{4} \mathrm{He}$ cryotarget operation. The numbers in parenthesis indicate parameters that were changed halfway through the experiment.

these density fluctuations happen on the same time scale as the $30 \mathrm{~Hz}$ helicity flip. In this case, there would be an additional contribution to the detector asymmetry width (discussed in section 3.6) of

$$
\sigma_{\text {meas }}^{2}=\sigma_{\text {stat }}^{2}+\sigma_{\text {fluct }}^{2}
$$

where $\sigma_{\text {stat }}$ is the width from counting statistics $(1 / \sqrt{N})$ and $\sigma_{\text {fluct }}$ is the increase due to density fluctuations in the target.

Heating at the target was controlled in two ways. A transverse flow of ${ }^{4} \mathrm{He}$ gas and $\mathrm{LH}_{2}$ allowed heat to be carried away quickly, and the beam was rastered over a $6.0 \mathrm{~mm}$ $\times 3.0 \mathrm{~mm}$ area incident on the target. The raster consisted of two dipole magnets, one vertical and the other horizontal, $23 \mathrm{~m}$ upstream of the target. It was driven by a $25 \mathrm{kHz}$ triangular waveform so that the beam was uniformly distributed over the target area [60]. Various estimates of density fluctuations were made by adjusting the raster size and and cryogen-loop fan speed and they were found to be controlled at about the $100 \mathrm{ppm}$ level, not large enough to be a significant problem [52]. 


\subsection{Hall A Spectrometers}

The Hall A Spectrometers have been used to investigate the structure of nuclei and nucleons. This requires a high resolution to be able to isolate the different reaction channels in nuclei and make a clean comparison with theory. A high luminosity is needed to achieve a good statistical accuracy. The present instrumentation in Hall A has been used with great success for measurements that require a high luminosity and high resolution in momentum and/or angle for at least one of the reaction products. The central elements are the two High Resolution Spectrometers (HRSs). Both of these devices provide a momentum resolution of better than $2 \times 10^{-4}$ and a horizontal angular resolution better than $2 \mathrm{mrad}$ with a design maximum central momentum of $4 \mathrm{GeV} / c[61]$.

\subsubsection{High Resolution Spectrometers}

The basic design of the Hall A HRSs is shown in Figure 4.10. The HRSs are based on a a superconducting magnet design with a QQDQ sequence: two quadropoles, a dipole with a $45^{\circ}$ vertical bend, and a third focusing quadropole to provide better resolution of the target's horizontal and angular coordinates. The vertical bend in the dipole provides a first order decoupling between the position along the target and the electron's momentum. The dipole $6.6 \mathrm{~m}$ long and has focusing entrance and exit face. Further focusing is achieved through the use of a field gradient in the dipole. The main design characteristics of the spectrometers are shown in Table 4.4. Both spectrometers were set to $12.5^{\circ}$, the minimum angle, to double the counting statistics and to provide some measure of left-right cancellation in helicity-correlated beam systematics. 


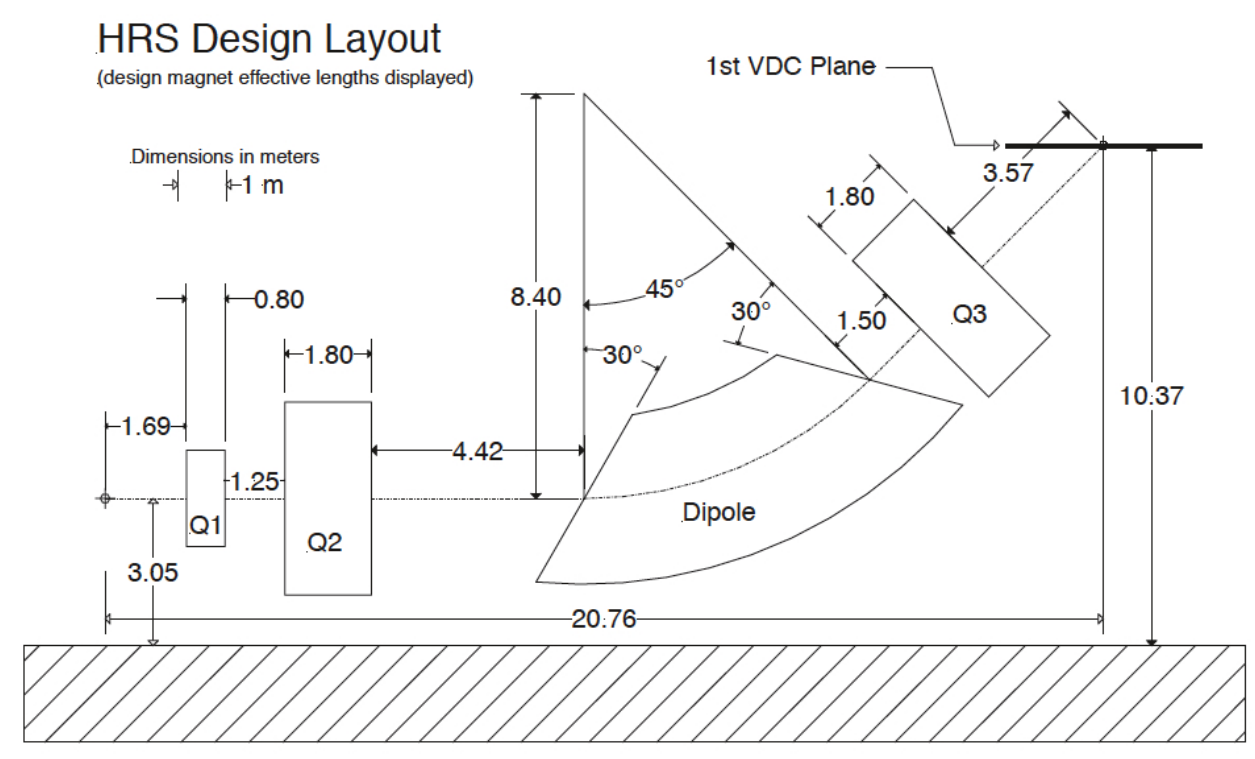

Figure 4.10: A schematic of the HRS spectrometer layout (side view) up to the first VDC plane.

The kinematics for HAPPEX require a $6^{\circ}$ scattering angle, and given the previously mentioned minimum of $12.5^{\circ}$, a pair of septum magnets [62] were installed to bend electrons scattered at $6^{\circ}$ out to the HRS acceptance at $12.5^{\circ}$. They were installed just upstream of the first HRS quadrupole and the scattering chamber was moved upstream by $0.8 \mathrm{~m}$ to make room for the installation. A diagram of the HRS setup with the septum installed can be seen in Figure 4.12.

The magnetic fields of the HRSs were set using the EPICS (Experimental Physics and Industrial Control System)* interface, and the central momentum of the spectrometer was chosen so as to focus elastically scattered electrons onto the focal plane of the detector. NMR probes measured the dipole fields while Hall probes monitored the quadrupoles; the Hall probes, however, were not stable over time and fields in the quadrupoles were based

${ }^{*}$ EPICS documentation, http://www.aps.anl.gov/epics 


\begin{tabular}{|c|c|}
\hline \hline Momentum range $p$ & $0.3 \sim 4.0 \mathrm{GeV} / \mathrm{c}$ \\
Configuration & QQDQ \\
Bend Angle & $45^{\circ}$ \\
Optical Length & $23.4 \mathrm{~m}$ \\
Dispersion (D) & $\pm 4.5 \%$ \\
Domentum Acceptance $\Delta p / p$ & $12.4 \mathrm{~cm} / \%$ \\
Radial Linear Magnification (M) & 2.5 \\
Momentum Resolution (FWHM) $\delta p / p$ & 5 \\
Angular Acceptance Horizontal & $1 \times 10^{-4}$ \\
Angular Acceptance Vertical & $\pm 28 \mathrm{mrad}$ \\
Solid Angle $\Delta \Omega$ & $\pm 60 \mathrm{mrad}$ \\
Angular Resolution (FWHM) Horizontal $\phi$ & $\sim 6.7 \mathrm{msr}$ \\
Vertical $\theta$ & $0.6 \mathrm{mrad}$ \\
Transverse Length Acceptance & $2.0 \mathrm{mrad}$ \\
Transverse Position Resolution (FWHM) & $\pm 5 \mathrm{~cm}$ \\
Spectrometer Angle Determination Accuracy & $1.5 \mathrm{~mm}$ \\
\hline \hline
\end{tabular}

Table 4.4: Hall A HRS general charateristics

instead on their current settings [61]. The momentum resolution of the HRSs allowed for a clean separation of elastic events from inelastic background making our integration technique possible. The detector footprint and simulated elastic peak focused onto the detector focal plane is shown in Figure 4.11.

\subsubsection{Septum Magnets}

The septum magnet field was also adjusted by setting the current on the EPICS interface. Each septum was made of superconducting coils with a cryogenically cooled iron yoke, and they were designed to have an acceptance of $24 \times 54 \mathrm{mSr}$ and provide a field up to $4.23 \mathrm{~T}$ while allowing an overall spectrometer $d p / P$ resolution of $1 \times 10^{-4}$. A blockage in the helium coolant circuit (from the time of manufacturing) and a leaky control valve [63] limited the flow in the right septum, and the current was set $+10 \%$ above the central momentum setting 


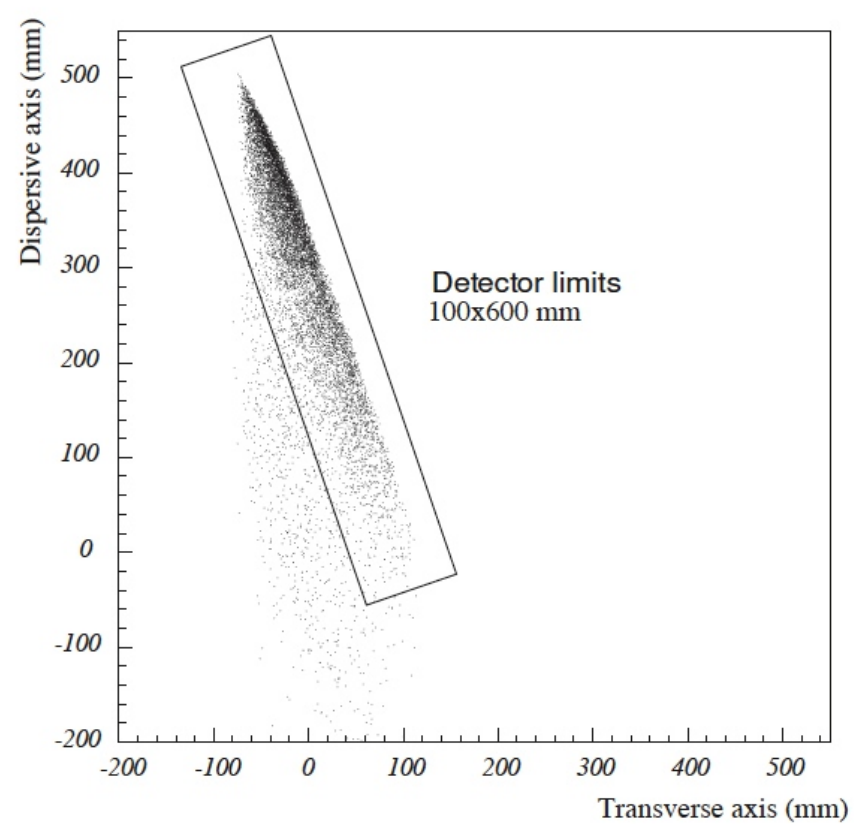

Figure 4.11: A simulated elastic peak focused onto the focal plane by the HRS. The detector footprint is shown by the rectangle in the diagram.

as this provided better cooling without disrupting the spectrometer acceptance. Low-energy Møller electrons caused some heating problems due to striking the bore of the magnet, and some tungsten collimators were designed to help reduce this, as well as some lead shielding placed upstream of each septum. Heating from the beam remained a problem throughout both the ${ }^{4} \mathrm{He}$ and ${ }^{1} \mathrm{H}$ run even though a "sweeper magnet" was built to sweep away low-energy Møller events toward the beampipe and away from the magnet bore [60].

\subsubsection{Hall A Standard Detector Package}

The standard detector package of Hall A consisting of Vertical Drift Chambers (VDCs) and scintillators were used in counting-mode for measuring $Q^{2}$, HRS optics, scattering angle, and backgrounds. The VDCs were used for reconstructing particle trajectories, and the 


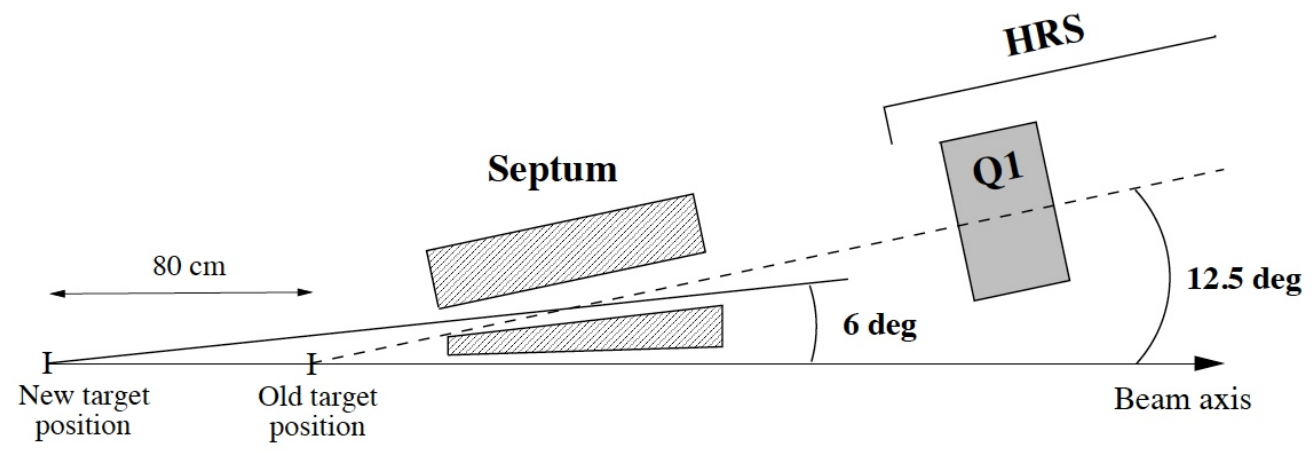

Figure 4.12: A view looking down on the HRS setup with the septum magnet installed.

scintillators were used for triggering. There was an insertable sieve-slit (Figure 4.13) located in a transfer box connecting the scattering chamber to the septum magnet that was used during optics studies for optimization.

The detectors were enclosed in a shield hut designed to protect the detectors from background radiation. Further shielding was provided by a Line-of-Sight block which was a two meter thick concrete block and was located on top of the first two quadrupoles in the HRS. The DAQ electronics were also placed in the shield hut to protect them from background radiation damage.

\subsubsection{VDCs}

Particle tracking for each HRS was provided by the two Vertical Drift Chambers (VDC) $[64,65]$ positioned $335 \mathrm{~cm}$ away from each other. The lower VDC was as close as possible to the focal plane of the spectrometer. Each VDC chamber was composed of two wire planes in a standard UV configuration - the wires of each successive plane were oriented $90^{\circ}$ to one another, and laid in the horizontal laboratory plane [61]. They were oriented at an angle 


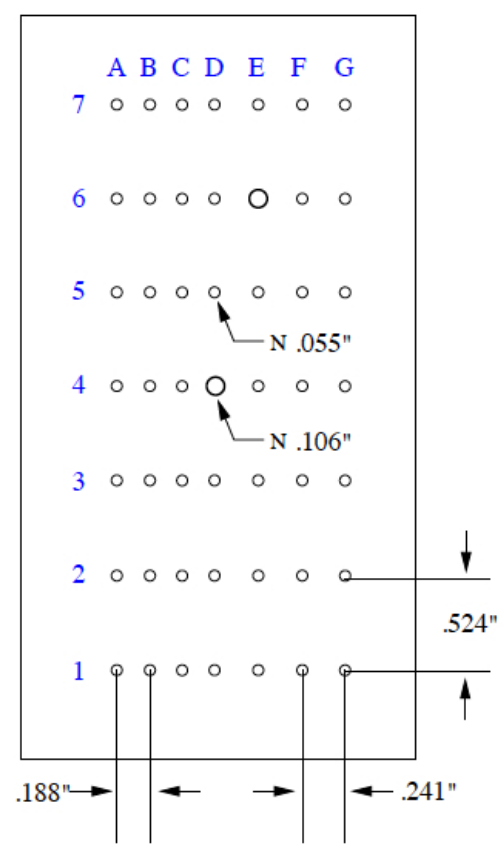

Figure 4.13: Sieve-slit hole pattern used for the optimization of spectrometer transfer matrices
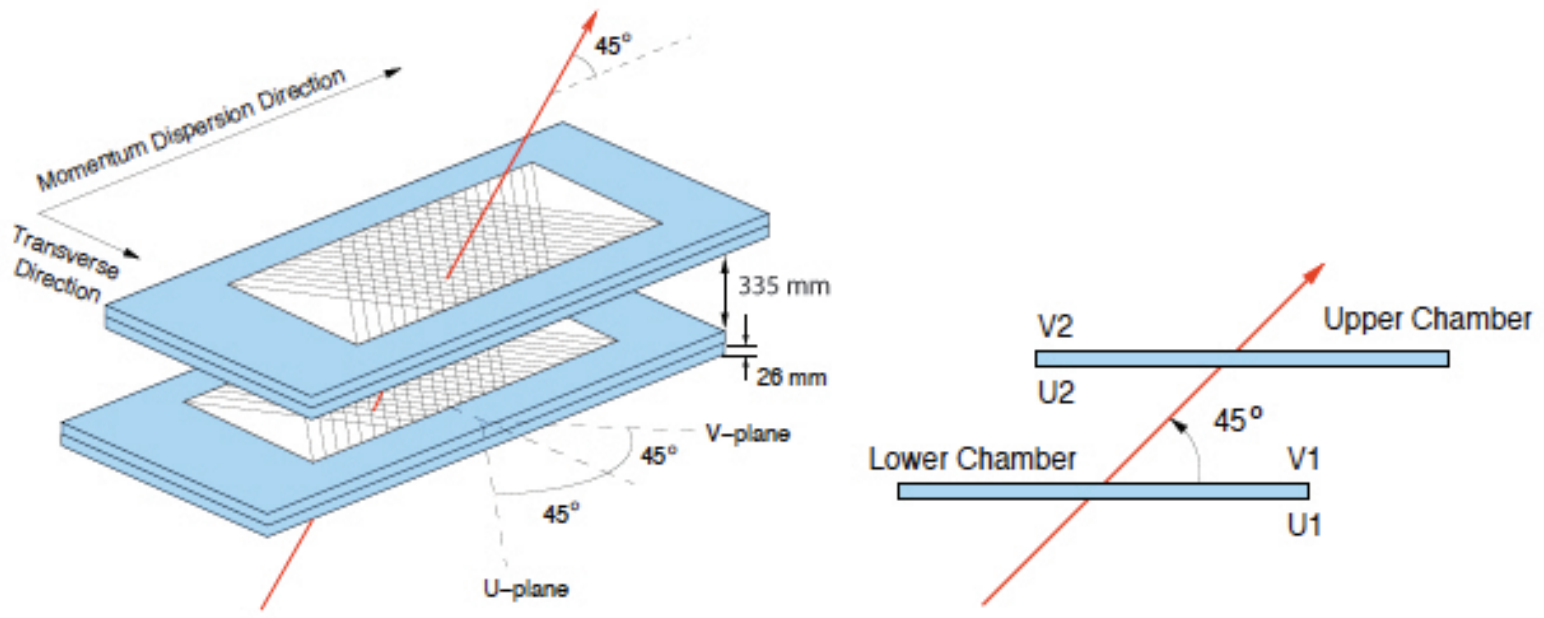

Figure 4.14: Schematic diagram (left) and side view (right) of wire chamber configuration in VDC planes 
of $45^{\circ}\left(-45^{\circ}\right)$ with respect to the dispersive and non-dispersive (transverse) directions as shown in Figure 4.14. The nominal particle trajectory crosses the wire planes at $45^{\circ}$.

Each VDC has three voltage plates at about $-4 \mathrm{kV}$, one between the $\mathrm{U}$ and $\mathrm{V}$ wire planes and two on opposite sides. The spacing was $26 \mathrm{~mm}$ between planes. The wires were kept at ground voltage so that the resulting electric field points from to the wires to the cathodes as displayed in Figure 4.15.

When a charged particle travels through a wire chamber, it ionizes the gas inside the chamber and leaves behind a track of electrons and ions along its trajectory. The gas mixture supplied to the VDCs was $62 \% / 38 \%$ argon-ethane $\left(\mathrm{C}_{2} \mathrm{H}_{6}\right)$ at a flow rate of 5 liter/hour per chamber [61]. The ionization electrons accelerate towards the wires along the path of least time (geodetic path). The time is measured by a Time-to-Digital Converter (TDC), which is started by the triggered wire and stopped by the event trigger supervisor. The idea behind particle tracking is as follows: Since the drift velocity of ionization electrons in the operating gas is known to be $50 \mu \mathrm{m} / \mathrm{ns}$, the distance from the particle's trajectory to each fired wire can be extracted from the corresponding TDC output. Combing the drift distance from all fired wires gives the trajectory of the charged particle. A charged particle entering the VDC at the nominal $45^{\circ}$ (the centered ray traveling through the HRS) will typically trigger five wires, while those at the extreme angle of $52^{\circ}$ will only trigger three [65]. The position resolution achieved in each direction was $100 \mu \mathrm{m}$ at full width half maximum (FWHM), and the angular resolution was $0.5 \mathrm{mrad}[61]$. 


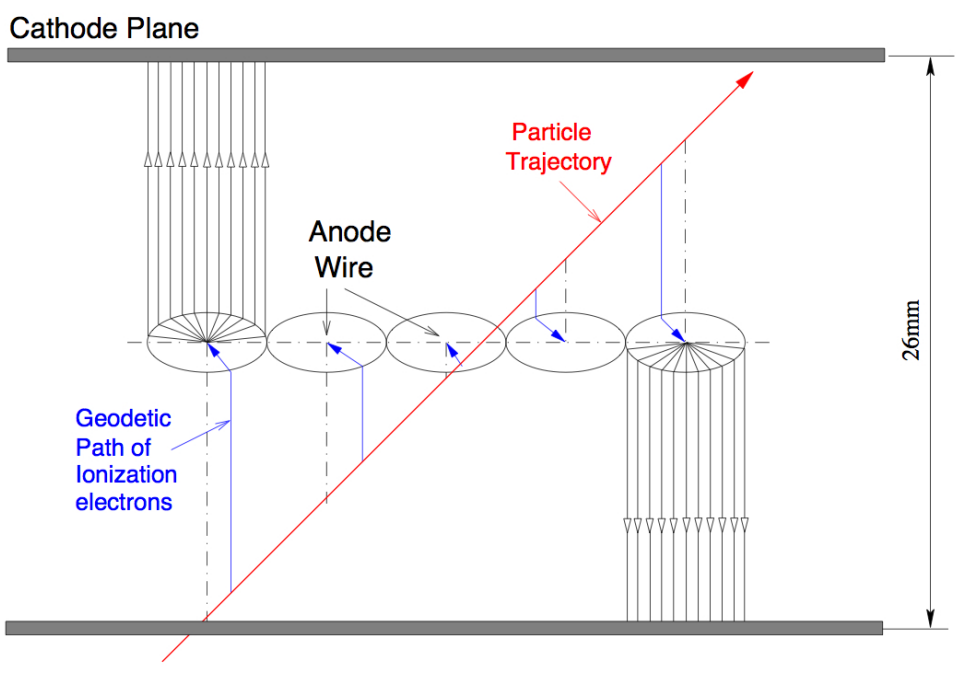

Figure 4.15: Configuration of wire chambers

\subsubsection{HAPPEX Focal-Plane Detectors}

The detectors used for HAPPEX were total absorption Cerenkov-shower calorimeters that collect the Cerenkov light using a photomultiplier tube (PMT), and integrate over each helicity window while allowing a high counting rate $(\sim 60 \mathrm{MHz})$ with no deadtime. The design of the detectors was modeled on the "sandwich design" used for the first generation HAPPEX experiment, but the forward-angle kinematics here made some design changes necessary.

Due to the high rate at such forward angles, the detector receives $\sim 1 \mathrm{MRad}$ of radiation dose; it was therefore necessary to use radiation-hard materials for the construction. 5 alternating layers of fused quartz (Spectrosil 2000) and brass were chosen to obtain a low energy resolution $(\Delta E / E$ of $17 \%[66])$, a resolution which can widen the asymmetry's statistical width by

$$
\sigma(A)=\frac{1}{\sqrt{N}} \sqrt{1+\left(\frac{\Delta E}{\langle E\rangle}\right)^{2}}
$$




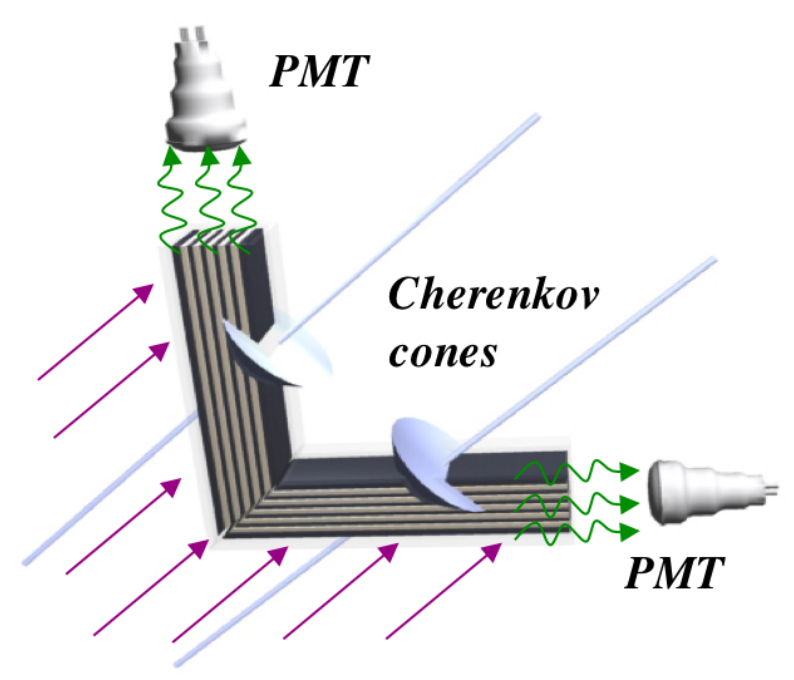

Figure 4.16: A drawing of the focal plane detector showing elastically scattered electrons entering from the right side. The Cerenkov light cone from the electromagnetic shower draw in the diagram is imagined.

where $N$ is the number of scattered electrons. The low energy-resolution contributes about $1.4 \%$ to the asymmetry width.

An area of $10 \mathrm{~cm}$ wide $\times 60 \mathrm{~cm}$ long is covered by the detector, dimensions chosen to overlap with the elastic scattering stripe, contain the electromagnetic shower, and exclude as much as possible inelastic events. An "L"-shape segmented detector design as shown in Figure 4.16 allowed for an asymmetry measurement at two near $Q^{2}$ values and provided a highly sensitive test of helicity-correlated beam asymmetries. Each segment was equal in length; the fused quartz in each segment was $1 \mathrm{~cm} \times 10 \mathrm{~cm} \times 30 \mathrm{~cm}$, and the brass layers were $1.5 \mathrm{~cm} \times 10 \mathrm{~cm} \times 30 \mathrm{~cm}$. Edge effects are the crucial drawback to this design; they contribute up to a $10 \%$ increase in the energy resolution; yet summing together the two detector signals before calculating the asymmetry makes crosstalk negligible. A cutaway view showing the quartz and brass layers of a detector segment in shown in Figure 4.17.

Each "L" -shaped detector sits about $3.5 \mathrm{~cm}$ above the focal plane in the HRS, and the 


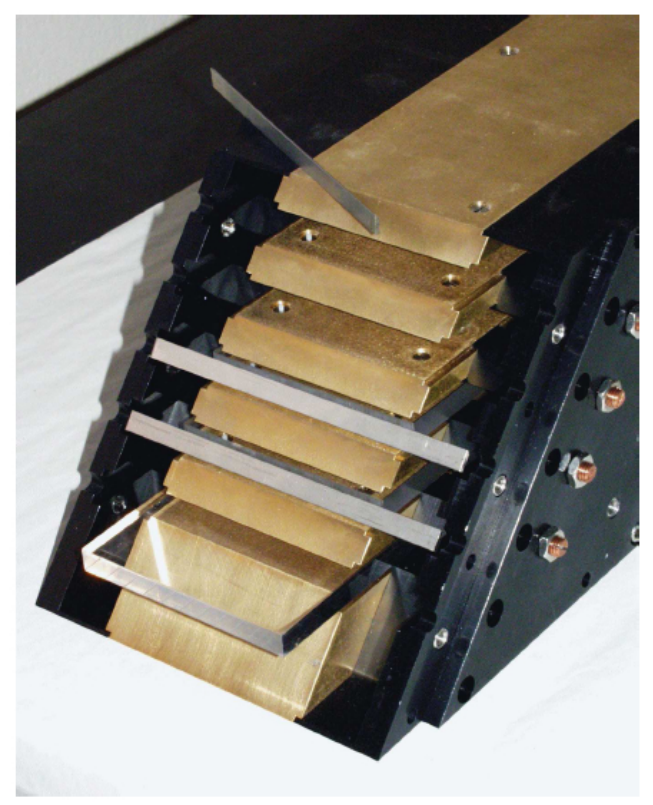

Figure 4.17: A cutaway view of the HAPPEX detector quartz and brass layers

detector segments on the left are labeled det1 and det2 while those on the right are assigned $\operatorname{det} 3$ and $\operatorname{det} 4 . \operatorname{det} 1$ and $\operatorname{det} 3$ are the low $Q^{2}$ detectors while $\operatorname{det} 2$ and $\operatorname{det} 4$ are those for measuring a bit higher $Q^{2}$. Linearity tests were run in June 2005 using LEDs, as well as tests during the run. Each test measured the detectors as linear to better than 1\% [67].

Scattered electrons reach the detector at $45^{\circ}$ with respect to the horizontal. The detectors as shown in Figure 4.16 are aligned along the C̆erenkov light cone which pens at $47.2^{\circ}$ to the horizontal. Light travels through the detector by internally reflecting off of the quartz. A $20 \mathrm{~cm}$ light guide connects each detector segment to the PMT. Additional details about the detector can be found in [68]. 


\section{S0 Detector}

There was one other detector used for HAPPEX called S0 that provided an unbiased trigger over the entire focal plane. This detector provided a means for identifying inelastic events that deviated from the expected elastic kinematics. This detector helped in better aligning the elastic peak on the HAPPEX detector, and provided a much better determination of the

${ }^{4}$ He quasielastic background. The background analysis and more on the S0 detector appear in Section 6.5.

\subsection{Data Acquisition}

The data for HAPPEX were acquired through two different methods. "Counting Mode", the standard Hall A data acquisition protocol, was used for the measurement of $Q^{2}$, alignment of the elastic peak onto the detector, and measurement of inelastically-scattered electrons contributing to background. "Integrating Mode" with the HAPPEX DAQ was used for the asymmetry data acquisition and the determination of aluminum target window background fraction. Both methods used the JLab DAQ software CODA which employed a VME controller running VxWorks to handle communication and the transfer of data between the DAQ and a PC running Linux.

The standard Hall A DAQ used the S2 scintillator plane and HAPPEX detectors to trigger the VDC readout, which provided reconstruction of the electron tracks between the focal plane and the target. A coincidence between two PMTs for any scintillator paddle defined the S2 trigger. The HAPPEx trigger was just a detected signal at a voltage over threshold. After a trigger was received, integration gates and common stops were created 


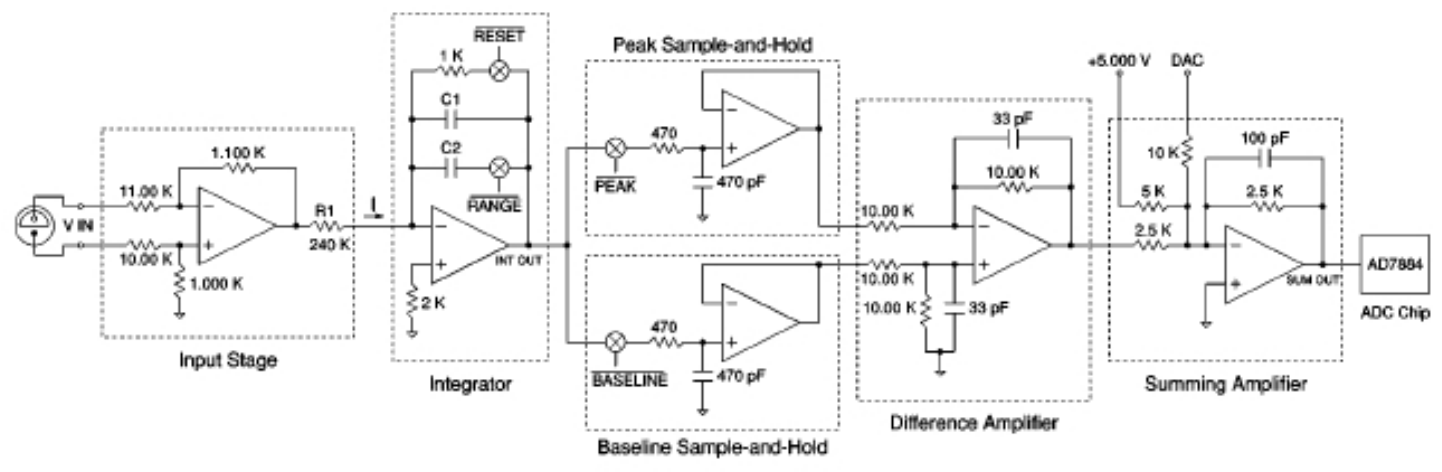

Figure 4.18: Circuit diagram of one channel of an integrating ADC

for the ADCs and VDCs, respectively [48]. Digitiized values were read out for each detector, and the necessary information was then encoded onto a storage disk. A circuit diagram of one channel of a 16 bit custom-made ADC is shown in Figure 4.18.

The HAPPEX DAQ integrated and digitized signals from the detectors and beam monitors, and was built of high resolution (16-bit) ananlog-to-digital converters (ADCs) and some ad-hoc ADCs constructed from voltage-o-frequency converters coupled with gate scalers (V2Fs). It was triggered at $30 \mathrm{~Hz}$ and synchronized to the helicity signal; the integration, however, only began at the end of a $500 \mu$ s pulse that marked the end of instability in the helicity signal that occurred as the Pockels cell was flipped from one state to the other. Since there exists a possibility of electronics pickup from the helicity signal, no helicity information was sent to the four VME crates that contained the integrating electronics. The helicity information sent was delayed by 8 windows and was sent to only the injector and counting-house crates. The other two for the LHRS and RHRS were without any sort of helicity signal.

With a simple hardware change to the electronics board, the ADCs can be switched from 
voltage to current integrating mode. Detectors are read out from current integrating ADCs while beam monitors are read out by voltage integrating ADCs or V2Fs. Measurements of helicity pickup in the electronics were made through several ADC channels connected to batteries. Further descriptions of the electronics can be found here [48, 46].

\subsection{Polarimetry}

The parity-violating asymmetry is defined assuming a 100\% polarized beam. Since such an ideal beam does not exist in reality, the experimental physics asymmetry becomes

$$
A_{\text {exp }}=\frac{A_{\text {corr }}}{P_{b}}
$$

where $A_{\text {corr }}$ is the raw asymmetry corected from beam asymmetries and $P_{b}$ is the beam polarization; $P_{b}$ is defined as

$$
P_{b}=\frac{N_{\uparrow}-N_{\downarrow}}{N_{\uparrow}+N_{\downarrow}}
$$

where $N_{\uparrow}\left(N_{\downarrow}\right)$ is the number of electrons aligned parallel (antiparallel) to the direction of beam propagation. The instruments used to measure the beam polarization for HAPPEX were based on Møller and Compton scattering, respectively.

\subsubsection{Møller Polarimeter}

A "Møller polarimeter" measures the asymmetry in the scattering of two polarized electrons $\left(\vec{e}+\vec{e} \rightarrow e^{\prime}+e^{\prime}\right)$. The scattering cross-section in the relativistic limit depends on the beam and target polarization as well as the Møller scattering analyzing power. A ferromagnetic 

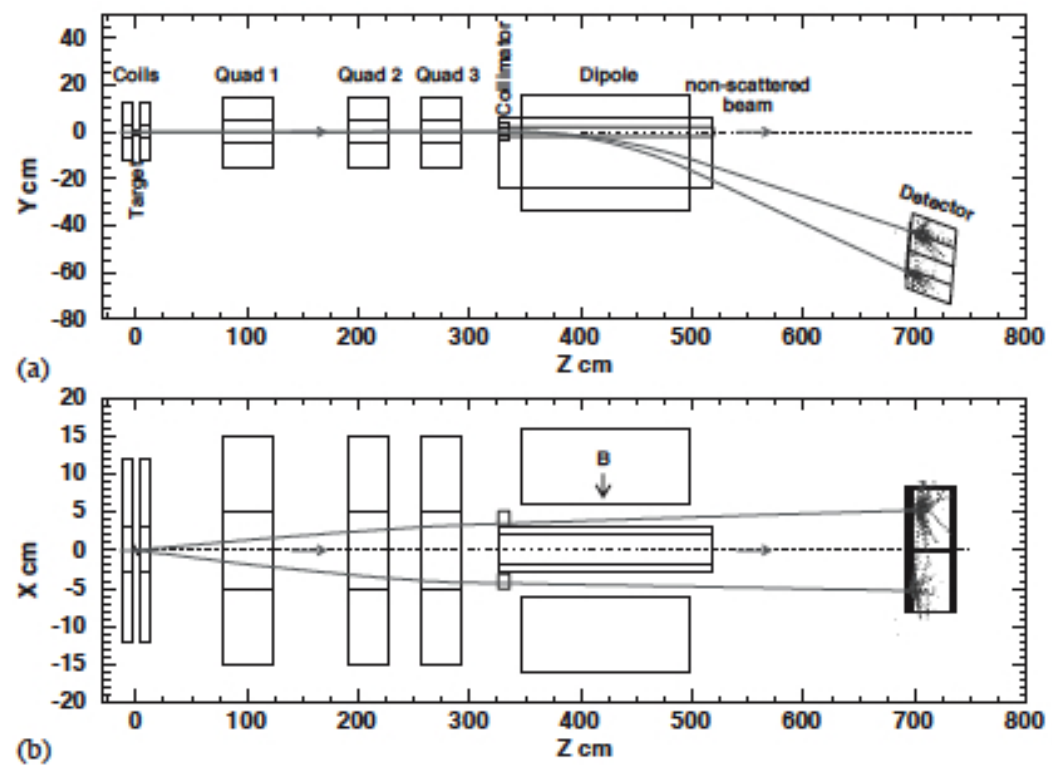

Figure 4.19: A layout of the Møller polarimeter presented from (a) a side view and (b) a top view. The displayed trajectories are from a Møller scattering simulation with a beam energy of $4 \mathrm{GeV}$ at $\theta_{C M}=80^{\circ}$ and $\phi_{C M}=0^{\circ}$.

foil target was polarized in a $24 \mathrm{mT}$ holding field produced from a set of Helmholtz coils, and its orientation could be adjusted to measure either longitudinal or transverse beam polarization. A layout of the Møller polarimeter is shown in Figure 4.19 [61].

The scattered electrons were focused onto the Møller detector using a series of 3 quadrupole magnets and a single dipole. They were detected in a pair of lead-glass calorimeters where the helicity-correlated detected rate was used to calculate the beam polarization. The average of the measurements from two target foils were averaged which helped to cancel transverse contributions as well as helicity-correlated beam asymmetries.

The Møller measurment needed to be done at very low currents making it inherently invasive, and it took an hour to achieve $0.2 \%$ statistical accuracy. The final systematic error from the Møller was 3\% and most of this was from uncertainty in the target polarization. Further description of the Møller polarimeter can be found in [61]. 


\subsubsection{Compton Polarimeter}

Compton polarimetry allows for a continuous, noninvasive measurement of beam polarization using the spin-dependent Compton scattering cross-section from QED. This polarimeter measures the asymmetry in scattering polarized electrons off of circularly polarized photons defined as

$$
A_{\exp }=\frac{N_{+}-N_{-}}{N_{+}+N_{-}}=A_{c} P_{\gamma} P_{e}
$$

as a way of measuring the beam polarization $P_{e}$ where $N_{+}\left(N_{-}\right)$is the Compton counting rate for right (left) helicity electrons normalized to beam intensity, and $A_{c}$ is a calculation of the Compton cross-section with $P_{\gamma}$ being the photon polarization.

The electron beam is diverted into the "Compton chicane" (Figure 4.20) where it interacts with circularly polarized photons at the Compton interaction point. A Fabry-Pérot laser cavity [69] was used at the interaction point to obtain a high photon flux keeping a high signal-to-noise ratio and achieving $\sim 1 \%$ statistical accuracy within 30 minutes. Scattered electrons were deflected into an electron detector using a dipole magnet after the FabryPérot interaction point while backscattered photons were detected by an electromagnetic calorimeter [70]. The Compton measurement was done with both right and left circularly polarized light to limit helicity-corelated beam asymmetry effects in the measurement's systematic error. 


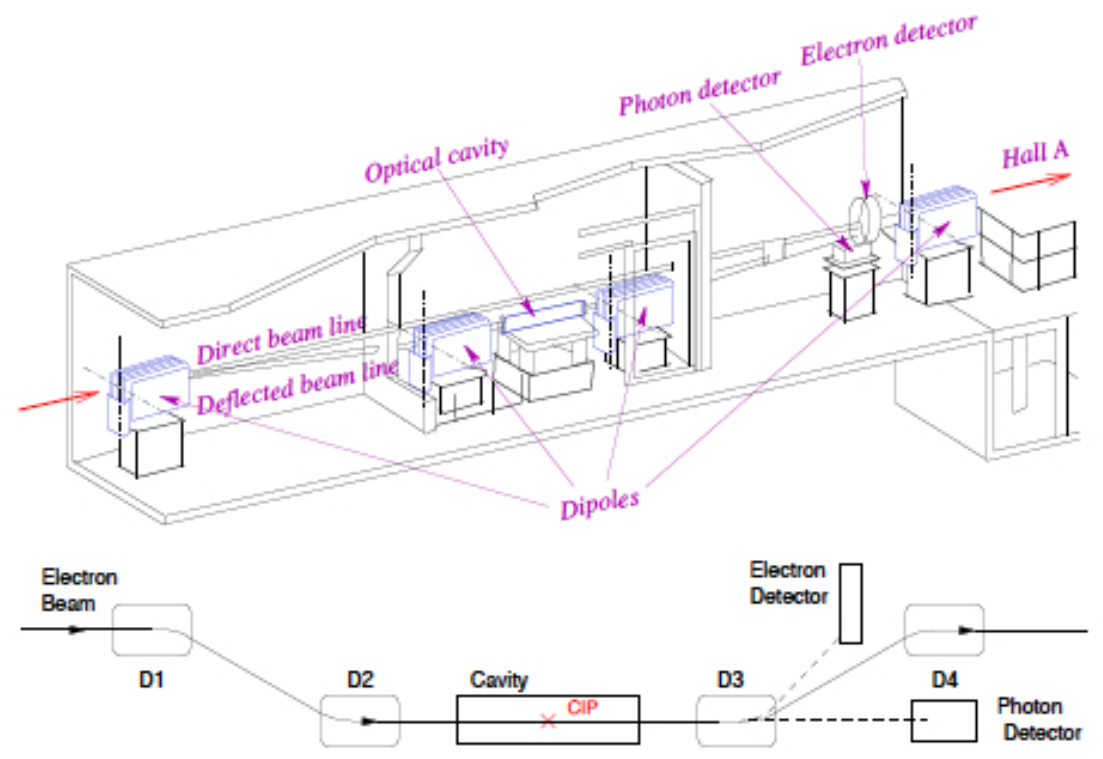

Figure 4.20: Layout of the Compton Polarimeter. The top diagram displays the polarimeter from the side while the bottom shows a view from above the compton chicane.

\section{8 $Q^{2}$ Profile Scanner}

Since $Q^{2}$ varies over the spectrometer acceptance, it is important to know the collected data's average $Q^{2}$ with good accuracy. A profile scanner was built by UMass designed to operate at the beam currents used during production running and was used to check whether the $Q^{2}$ distribution at low current matches well with that at production conditions. The scanner was used in integrating mode to measure the $Q^{2}$ distribution approximately once a day during production running.

The detector in the scanner was a $20 \mathrm{~mm} \times 5 \mathrm{~mm} \times 5 \mathrm{~mm}$ quartz block inclined at $45^{\circ}$ to the electron beam. There was a tungsten radiator approximately $15 \mathrm{~mm}$ thick in front of the quartz block; light from the quartz was directed down a light guide with Alzak, for high reflectivity, down to a single PMT [49]. A schematic of the scanner is shown in Figure 4.21. 


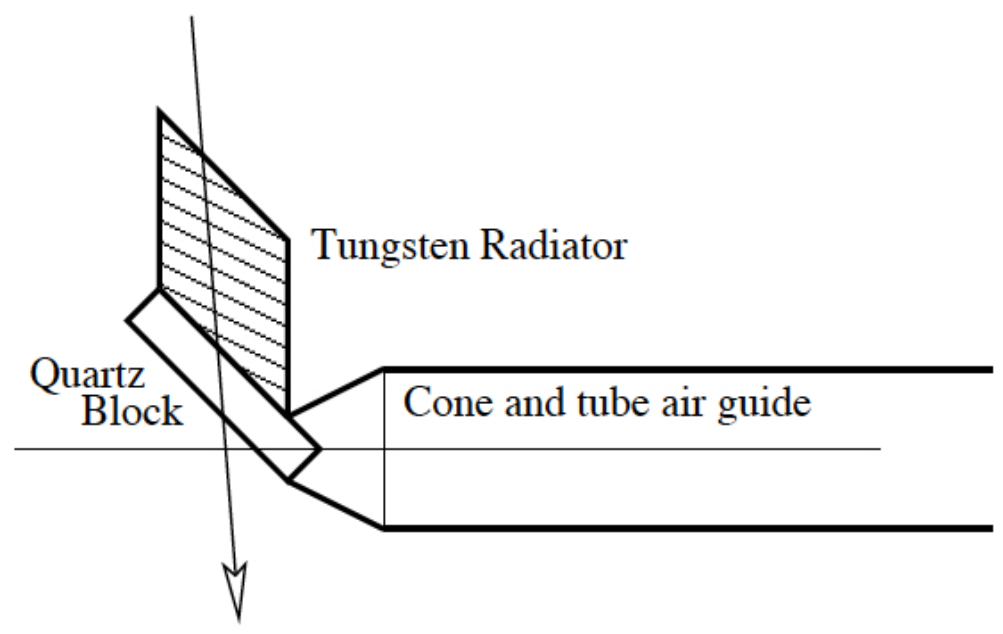

Figure 4.21: A schematic of the $Q^{2}$ profile scanner.

A scanner in each spectrometer arm was placed on two Parker-Daedal 406XR drives aligned perpendicularly with one another to allow the entire focal plane to be scanned in the transverse $(y)$ and dispersive $(x)$ directions. LabView controlled the driver motion remotely, and each drive was fitted with a UniMeasure LX-PA linear potentiometer (0-10 V) for real-time position readout. These scans provided a map of the incoming flux over the focal plane, and it was verified that this distribution remained constant throughout the HAPPEX run. 


\section{Chapter 5}

\section{Polarized Electron Source}

The polarized electron source at Jefferson Lab was designed to generate highly circularly po-

larized light while preventing helicity-correlated beam asymmetries (referred to as beam $A_{\mathrm{LR}}$ 's from here on) from causing false asymmetries in the parity-violating measurements at a significant level. A major effort before the 2005 HAPPEX run was to understand the source and minimize the effects of these mysterious helicity-correlated position differences in the laser beam, and consequently, in the electron beam.

This source had its origin in the Pockels cell's birefringence and how it affected a Gaussian laser beam traveling through the cell. Light traveling through a birefringent material will acquire relative shifts in phase between its orthogonal components of polarization. If the light is incident on the material at different angles across the beam spot, a gradient in phase across the beam spot will result, as the phase retardation depends on the angle of the light's polarization components with the material's indices of refraction. It turned out that the resulting gradient in phase across the Gaussian beam spot depended on the angle of 
incidence (named "skew angle") between the laser light and the surface of the Pockels cell; it was aptly named the "Laser Skew Effect". This chapter will discuss the efforts at Jefferson Lab to both understand and minimize this effect and many others due to beam $A_{\mathrm{LR}}$ 's. The source setups for helium $\left({ }^{4} \mathrm{He}\right)$ and hydrogen $\left({ }^{1} \mathrm{H}\right)$ will be discussed in the last section.

\subsection{Circular Polarization and Setup of the Pockels Cell}

This section will briefly describe some of the formalism regarding circular polarization, as well as provide a description of the optics and particularly the Pockels cell, that are used to circularly polarize the laser light. Imperfections to the laser beam's circular polarization will create components of linear polarization and/or changes in the component of linear polarization across the beam spot (phase gradients) that will ultimately contribute to beam $A_{\mathrm{LR}}$ 's. Beam-steering effects may also contribute to such asymmetries. The sources of these beam $A_{\mathrm{LR}}$ 's will be described in detail in section 5.2 , but the sensitive setup of the Pockels cell will be described here.

\subsubsection{Circular Polarization}

The purpose of our optics setup was to generate highly circularly polarized light of both helicities while minimizing beam $A_{\mathrm{LR}}$ 's. We had one Pockels cell in our setup that acted as a quarter-wave plate with its fast and slow axes oriented at $45^{\circ}$ to the horizontal. A voltage was applied along its length to shift the components of incident laser light along the fast and slow axes one quarter-wave $\left(90^{\circ}\right)$ relative to one another to produce circularly polarized light. The Pockels cell used in both our studies and during the experimental run was $20 \mathrm{~mm}$ 
in length and manufactured by Cleveland Crystals in Highland Hts., OH (QX2035 KD*P). The sign of the phase retardation (and hence helicity state) was chosen pseudorandomly on a pulse-by-pulse basis, and the appropriately applied quarter-wave voltage was adjusted to compensate for additional components of linear polarization that originated from residual birefringence in the Pockels cell or phase retardations in other optical elements. The phase shift $\delta_{P C}$ induced by the Pockels cell (PC) as a function of the applied voltage to it is given by the expression

$$
\delta_{P C}=\frac{\pi}{V_{\lambda / 2}} V_{P C}
$$

where $V_{\lambda / 2}$ is the voltage required for half-wave retardation $(\sim 5.4 \mathrm{kV})$ and $V_{P C}$ is the voltage applied across the length of the cell, approximately $\pm 2.7 \mathrm{kV}$ for circularly polarized light.

Phase shifts such as $\pi / 2 \pm \varepsilon$ that deviate slightly from perfectly circular polarized light can generate significant linear components that cause beam $A_{\mathrm{LR}}$ 's. The degree of linear and circular polarization present in a beam of laser light are represented by the expression

$$
L^{2}+C^{2}=1
$$

where $L$ and $C$ represent the fractional compositions of linear and circular light, respectively. This addition in quadrature of the two components means small phase shifts away from quarter-wave can have little effect on the circular component but a large one on the linear component. This linear component is what we hope to minimize in aligning the Pockels cell. 


\subsubsection{Alignment of the Pockels Cell}

In aligning and positioning the Pockels cell, it is important where the laser light enters and passes through the cell. Given this, the cell was screwed into a mount that could translate in both axes orthogonal to the beam direction, and was also adjustable in pitch, yaw, and roll. Proper alignment in pitch and yaw was achieved when maximum extinction was measured downstream of the Pockels cell when it was placed between two crossed linear polarizers; this means the ratio of power transmitted through aligned polarizers to that transmitted through crossed ones was as high as possible. This was achieved by adjusting pitch and yaw on the Pockels cell mount. To avoid the possibility of aligning the cell in the orientation of a false minima, a method involving the use of an isogyre pattern can be used [71]. The idea here is that a properly aligned Pockels cell should affect a passing laser beam as little as possible; one aligned improperly would add an orthogonal component of polarized light and lessen the extinction ratio.

After this initial alignment, we checked to see how much linear polarization was present on the laser beam after exiting the Pockels cell. This was done by adding a spinning linear polarizer (SLP) downstream of the Pockels cell. This was simply a linear polarizer which had a motor, allowing the polarizer to be spun about the direction of laser beam propagation. Laser light could travel through this polarizer and it was collected on the other side by a power meter. The power meter was connected to an oscilloscope which allowed us to see the oscillations in power as the SLP was rotated. The larger the oscillation, the larger was the linear polarization on the laser beam. The Pockels cell could be rotated about it center (called "roll") and the applied voltages could be adjusted to minimize these oscillations. This 
procedure minimized the linear polarization and tuned the Pockels cell to behave strictly as a quarter-wave plate.

The previously mentioned mysterious source of position differences that was later found to originate from a nonzero angle of incidence of the laser light with the surface of the Pockels cell was then minimized. Adjustments to this angle could be made by adjusting the pitch and yaw position of the Pockels cell with the Pockels cell mount, and scans along each of these dimensions were performed find the best pitch and yaw positions to minimize the skew angle. This procedure is described in more detail in Section 5.3.2. Scans across the in the $\mathrm{x}$ and $\mathrm{y}$ dimension of the Pockels cell were also needed to find the geometric center of the cell and limit contributions from steering and birefringence. The following sections will discuss some of these and further enhancements to the alignment and other techniques needed to minimize beam $A_{\mathrm{LR}}$ 's.

In addition to these methods, the size of the laser beam spot at the Pockels cell is believed to be important as effects related to angles of divergence and dependence on spot size of birefringence gradients contribute to beam $A_{\mathrm{LR}}$ 's. The size of the beam spot was controlled by the presence of a beam telescope consisting of two concave lenses placed some distance downstream from the laser aperture. The size of the beam spot at the Pockels cell, however, had to be balanced with the constraints on the beam size at the cathode. The typical beam diameter at the cathode was $\sim 1 \mathrm{~mm}$. Within a Gaussian laser beam's Rayleigh range, the beam envelope's divergence is smaller than it is outside this range [72]; and the integrated average of a beam's divergence over all photon angles may be smaller for a more tightly collimated beam. This is important as light rays with smaller angles will correspond to 
smaller phase shifts after passing through the Pockels cell crystal. This idea needs more investigation as it has not been rigorously explored, and it is difficult to know exactly how photon angles are distributed within a Gaussian beam as a function of spot size or distance from the beam waist. This is one caveat regarding our approach in trying to develop an analytical formalsim for the "Laser Skew Effect" (discussed in Section 5.2.4) Also, there are some rudimentary calculations suggesting that the magnitude of position differences due to birefringence gradients depend on beam spot size. Some combination of a more tightly collimated beam with a smaller beam diameter would likely allow for improvements in the source's performance, but a rigorous analytical formalism has not been developed and there are still questions about how technically feasible future improvements could be on this front.

\subsection{Sources of Helicity-Correlated Beam Asymmetries}

This section discusses the various sources of helicity-correlated beam asymmetries ( ${ }^{\text {beam }} A_{\mathrm{LR}}$ 's); a newly discovered one involving a non-zero angle of incidence of the laser light with the optic axis of the Pockels cell is discussed in section 5.2.4.

\subsubsection{Polarization Induced Transport Asymmetry (PITA)}

The interaction of the helicity-correlated changes in a laser beam's residual linear polarization with a cathode's QE anisotropy is usually the dominant way of generating a helicitycorrelated asymmetry in a polarized electron beam's intensity. A laser beam's linear component of polarization arises from the following sources: a non-zero angle of incidence of the laser light with the optic axis of the Pockels cell, birefringence in the Pockels cell, or 
birefringence in the optical elements between the Pockels cell and the cathode.

These effects produce phase shifts which add components of linear polarization to a circular polarized laser beam. When this linear polarization interacts with the cathode's QE anisotropy, significant helicity-corrleated variations in the electron beam's intensity are produced. This effect has been referred to as a Polarization-Induced Transport Asymmetry (PITA) [73] and can be a significant source of beam $A_{\mathrm{LR}}$ 's. A derivation of PITA for the SLAC polarized source [74] applies well for the source at Jefferson Lab and will be used in what follows to provide the analytical formalism necessary for a more rigorous description of this effect.

We first allow a Pockels cell to induce retardations that provide a fully general description of elliptically polarized light. So that the asymmetry has a simple form, we choose a particular way to write them:

$$
\delta_{R}=-\left(\frac{\pi}{2}+\alpha\right)-\Delta \quad \delta_{L}=+\left(\frac{\pi}{2}+\alpha\right)-\Delta
$$

where R, L indicate right- and left-helicity light and $\alpha$ and $\Delta$ represent the imperfect respective "symmetric" and "antisymmetric" phase shifts such that $\alpha=\Delta=0$ corresponds to perfectly circularly polarized light. The reason for the names "symmetric" and "antisymmetric" is apparent from Figure 5.1. A nonzero symmetric phase shift $(\alpha)$ turns circular polarization into elliptical where both helicities share the same major and minor axes, i.e., the phase shift afects the two polarization ellipses symmetrically. A nonzero $(\Delta)$ phase, however, results in elliptical polarization for which the two polarization ellipses have their major and minor axes interchanged, an antisymmetric behavior. 


$$
\begin{aligned}
& \alpha \neq 0 \\
& \Delta=0
\end{aligned}
$$

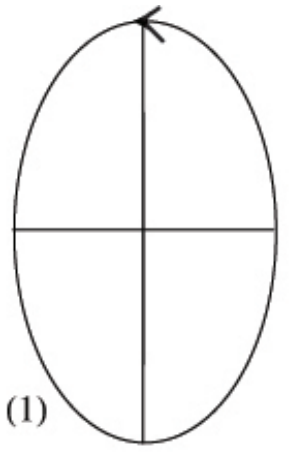

$$
\begin{aligned}
& \alpha=0 \\
& \Delta \neq 0
\end{aligned}
$$

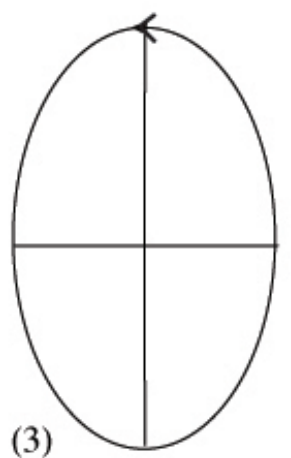

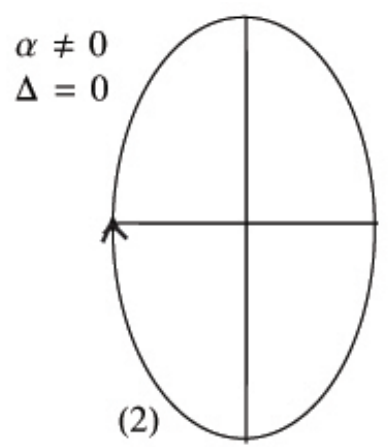

$\alpha=0$

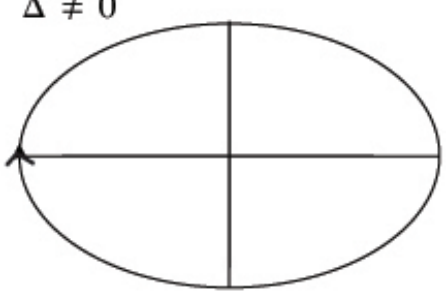

(4)

Figure 5.1: (1) and (2) display the polarization ellipses for right- and left-helicity light, respectively, from the addition of a nonzero symmetric phase shift, $\alpha$. (3) and (4) show the right- and left-helicity ellipses, respectively, from the addition of a nonzero antisymmetric phase shift, $\Delta$.

The parameterization given in Equation 5.3 is a completely general description of the elliptical polarization reaching the cathode, where additional phase shifts from components downstream of the Pockels cell can be included as additional contributions to $\alpha$ and $\Delta$.

After defining the transmission coefficients along some axes $x^{\prime}$ and $y^{\prime}$ as $T_{x^{\prime}}$ and $T_{y^{\prime}}$, the intensity of light after transport through a Pockels cell and asymmetric transport element can be written as

$$
I_{R(L)}=\vec{E}_{f}^{*} \cdot \vec{E}_{f}=T^{2}+\frac{\varepsilon^{2}}{4}+\varepsilon T \cos \delta_{R(L)} \cos 2 \theta
$$

where $T=\left(T_{x}^{\prime}+T_{y}^{\prime}\right) / 2, \varepsilon=T_{x}^{\prime}-T_{y}^{\prime}$, and $\theta$ is the angle between $x^{\prime}$ and the horizontal axis. 
Finally, we calculate the asymmetry in beam intensity $A_{Q}$ by first writing $\delta_{R(L)}$ in Equation 5.4 in terms of $\alpha$ and $\Delta$. We then used the small angle approximation and only keep terms that are first order in phase shifts and first order in $\varepsilon$ :

$$
A_{Q}=\frac{I\left(\delta_{R}\right)-I\left(\delta_{L}\right)}{I\left(\delta_{R}\right)+I\left(\delta_{L}\right)}=-\frac{\varepsilon}{T}\left[\left(\Delta-\Delta^{0}\right)\right] \cos 2 \theta
$$

where $\Delta^{0}$ represents any residual birefringence in the Pockels cell or downstream optics thereof, and $\varepsilon / T$ is the analyzing power. Note here that birefringence in downstream optics can only contribute antisymmetric $(\Delta$-type) phase shifts. The above formalism assumes that the asymmetric transport element is a component of the optical system. Whether the analyzing power is provided by a linear polarizer in the laser room or the QE anisotropy of a superlattice cathode, the above formalism remains. The superlattice cathodes in use at Jefferson Lab remain the dominant analyzing power in the system.

\subsubsection{PITA Slopes}

In Equation 5.5 above, notice that $A_{Q}$ depends linearly on $\Delta$. An easily measurable quantity, referred to here as the PITA slope, characterizes the sensitivity of the source to the residual linear polarization of the system. From Equation 5.5, we define

$$
m=-\frac{\varepsilon}{T} \cos 2 \theta
$$

The $\Delta$ phases can be converted to voltages as was previously discussed for all $\delta_{(R) L}$ phase shifts induced by the Pockels cell (Equation 5.3). These voltages can adjust the laser light's 


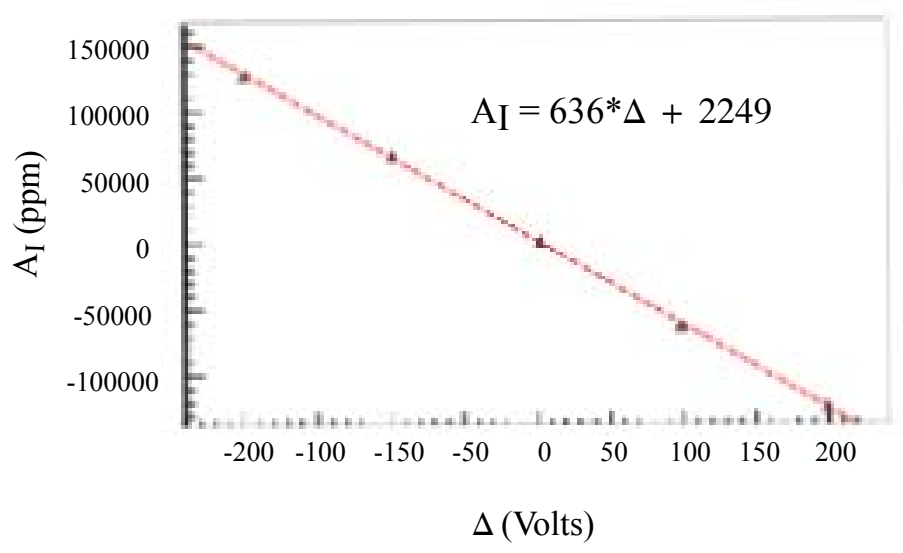

Figure 5.2: $A_{Q}$ measured versus offset voltage, $\Delta$.The PITA slope of $636 \mathrm{ppm} /$ Volt is typical of that measured on the laser table with the analyzing power provided by a linear polarizer.

circular polarization and affect $A_{Q}$. Measuring the PITA slope by applying offset voltages can thus help to minimize ${ }^{\text {beam }} A_{\mathrm{LR}}$ 's. Figure 5.2 shows the results of such a measurement where the y-intercept on the plot reveals how far the source is from optimal polarization and this intercept divided by the slope indicates how much adjustment the voltages need to move there. In Fig. 5.2, a movement in Pockels cell voltages of $2249 / 636 \approx-3.5$ Volts is needed to zero $A_{Q}$ and optimize the beam's circular polarization.

\subsubsection{Spatial Variation of $\Delta$}

Until now, we have developed only the idea of beam $A_{\mathrm{LR}}$ 's being generated from an antisymmetric phase shift $(\Delta)$ as evident in Equation 5.5, one that is constant across space. We must next consider an optical element generating a spatial variation of $\Delta$ across a laser beam and the beam $A_{\mathrm{LR}}$ 's that result. If $\Delta$ varies in space, $A_{Q}$ must also (Equation 5.5), and such spatial variations allow for the possibility of higher-order beam $A_{\mathrm{LR}}$ 's.

For example, a linearly varying $\Delta$ leads to a linearly varying $A_{Q}$. A horizontal linear 

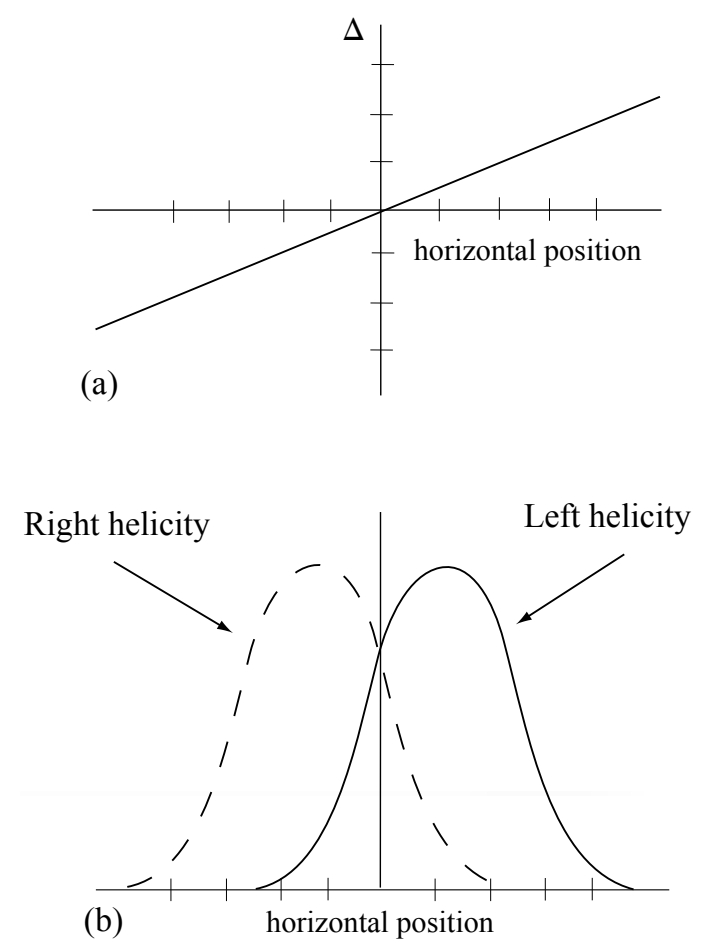

Figure 5.3: A linear gradient in $\Delta$ results in helicity-correlated shifts in an electron beam's intensity profile (a) $\Delta$ varying horizontally across laser beam face (b) The resulting horizontal shifts for rightand left-helicity electron beams after interacting with an analyzing power in the beamline 
variation of this phase as in Fig. 5.3a will shift the centroids of the right- and left-helicity electron beams in opposite directions and produce helicity-correlated horizontal position differences (Fig. 5.3b). Such effects are present in the Pockels cell, either due to birefringence gradients or the nonzero angle of incidence of the laser light with the Pockels cell's optic axis.

These position differences are among the higher-order beam $A_{\mathrm{LR}}$ 's of the electron beam, other such examples include spot size and shape. Such asymmetries can be characterized by "moments" similar to the moments of a statistical distribution. The zeroth moment (the average phase shift across the beam) gives rise to $A_{Q}$; the first is related to the gradient in phase across the laser beam and gives rise to $D_{X(Y)}$; the second is related to the curvature of the phase and gives rise to spot size differences; and even higher-order moments give rise to other asymmetries in the beam profile. A "moment" calculation characterizing our newly discovered source of position differences, a non-zero angle of incidence of the laser beam with the optic axis of the Pockels cell, is described in the next section.

\subsubsection{Laser Skew Effect}

There was a dominating source of position differences discovered on the laser table that was minimized during the injector tunnel setup for both the $\mathrm{He}$ and $\mathrm{H}$ runs. This source arose from a small nonzero angle of incidence of the laser light with the optic axis of the Pockels cell (the axis running through its center and perpendicular to both faces) as shown in Figure 5.4. Ideally, when aligning the Pockels cell, this angle will be zero. However, there are limits to how well this can be achieved when large position differences are created due to residual linear polarization on the laser beam that appeared to be minimized when 
aligning the cell. It appeared that our procedure using just the "spinning linear polarizer" (SLP), power meter, and oscilloscope was too crude to minimize this effect. Alignment of the Pockels cell and minimization of beam $A_{\mathrm{LR}}$ 's will be discussed in section 5.5 , but the development of the analytical formalism needed to more fully describe and quantify this newly discovered source of position differences is presented here. This formalism will discuss specifically position differences as measured on the laser table but could easily apply to position differences in electron beams produced from the interaction of a $\Delta$ phase gradient with the analyzing power of a cathode.

In constructing a such a formalism, first consider the weighted mean for the position of each helicity distribution according to

$$
\bar{x}^{R(L)}=\frac{\sum_{i} I_{i}^{R(L)} x_{i}^{R(L)}}{\sum_{i} I_{i}^{R(L)}}
$$

where $x_{i}^{R(L)}$ is the position of the $i$ th collection of rays in a pulse of right- or left-helicity light and $I_{i}^{R(L)}$ is the intensity of that $i$ th element. Subtracting $\bar{x}^{L}$ from $\bar{x}^{R}$ while substituting the average intensity $\frac{I_{i}^{R}+I_{i}^{L}}{2}$ for $I_{i}^{R}$ and $I_{i}^{L}$ in the denominator of each expression yields

$$
\Delta x=\bar{x}^{R}-\bar{x}^{L}=\frac{\sum_{i} 2 A_{Q_{i}} \frac{I_{i}^{R}+I_{i}^{L}}{2} x_{i}}{\sum_{i} \frac{I_{i}^{R}+I_{i}^{L}}{2}}
$$

where $A_{Q_{i}}=\frac{I_{i}^{R}-I_{i}^{L}}{I_{i}^{R}+I_{i}^{L}}$ is the charge asymmetry between a right- and left-helicity ray of light.

On the laser table, the beam passed through a "cleanup" polarizer before the Pockels cell and was then analyzed by another polarizer downstream. Any optical components, such as Pockels cells, that retard light can be well approximated as different cases of the the unitary 
Jones matrix for a rotated retardation plate [75]:

$$
J_{R E T}=\left[\begin{array}{cc}
\cos ^{2} \gamma+e^{i \delta} \sin ^{2} \gamma & \left(1-e^{i \delta}\right) \sin \gamma \cos \gamma \\
\left(1 e^{i \delta}\right) \sin \gamma \cos \gamma & \sin ^{2} \gamma+e^{i \delta} \cos ^{2} \gamma
\end{array}\right]
$$

Here, $\gamma$ is the angel between the retarder's fast axis and the horizontal axis and $\delta$ is the retardation induced between the fast and slow axes.

The cleanup polarizer was oriented to transmit vertically linearly polarized light; thus it's initial electric vector can be represented as

$$
\vec{E}_{i}=\left[\begin{array}{l}
0 \\
1
\end{array}\right]
$$

The Pockels cell had its fast axis at $45^{\circ}$ from the horizontal $(\pi / 4)$, induces a retardation $\delta_{P C}$, and can be represented by the matrix

$$
J_{P C}=\frac{1}{2}\left[\begin{array}{cc}
1+e^{i \delta_{P C}} & 1-e^{i \delta_{P C}} \\
1-e^{i \delta_{P C}} & 1+e^{i \delta_{P C}}
\end{array}\right]
$$

where $\delta_{P C}$ is set close to $\pm \pi / 2$. The final polarization vector immediately following passage through the Pockels cell can be written as

$$
\vec{E}_{f}=J_{P C} \cdot \vec{E}_{i}
$$

and the intensity of the laser light, as we've already seen in Equation 5.4 , is $I_{R(L)}=\vec{E}_{f}^{*} \cdot \vec{E}_{f}$. The above formalism allows us to calculate the intensity of any transmitted ray of light, and 


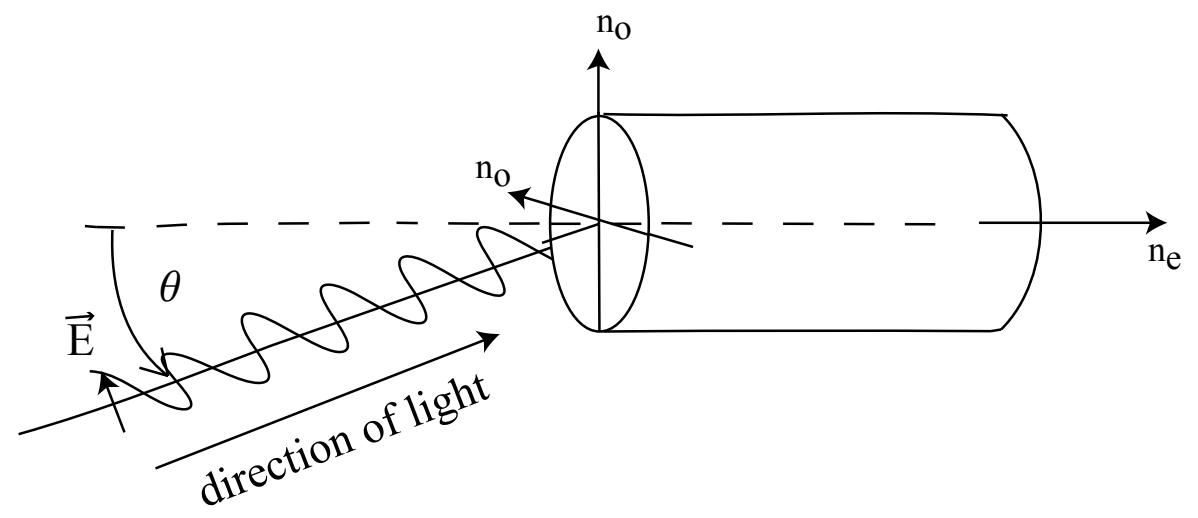

Figure 5.4: A ray incident at some angle $\theta$ with respect to the optic axis (labelled $n_{e}$ ) of the Pockels cell. This ray will see a superposition of indices of refraction $\left(n_{o}\right.$ and $\left.n_{e}\right)$ whereas a ray of normal incidence would see only $n_{o}$. A ray of orthogonal polarization to the one pictured would see only $n_{o}$ as well.

show that it will be of the form

$$
I_{i}^{R}=\frac{1}{2}(1-\alpha-\Delta) \quad I_{i}^{L}=\frac{1}{2}(1-\alpha+\Delta)
$$

for right- and left-helicity light, respectively. $A_{Q_{i}}=\frac{I_{i}^{R}-I_{i}^{L}}{I_{i}^{R}+I_{i}^{L}}$ is then equal to only the phase shift $\Delta$ with $\alpha \ll 1$.

To calculate $\Delta$, we must first consider the phase shift experienced by a ray incident at some skew angle with respect to the Pockels cell's optics axis as shown in Figure 5.4. The phase shift experienced by any ray passing through a material with two refractive indices is

$$
\Delta \phi=\frac{2 \pi d}{\lambda}\left(n_{1}-n_{2}\right)
$$

where $d$ in our case is the length of the Pockels cell, $\lambda$ is the wavelength of the laser light, $n_{1}=n_{o}\left(n_{o}=\right.$ the index of refraction along the ordinary axis), and $n_{2}$ is a superposition of $n_{o}$ and $n_{e}\left(n_{e}=\right.$ the index of refraction along the extraordinary axis). From Figure 5.4, 
any ray incident at some angle $\theta$ with respect to the Pockels cell's optic axis can be broken down into two orthogonal components, one that is only refracted by $n_{o}$ and another by both $n_{o}$ and $n_{e}$. This superposition is written as $\frac{1}{n_{2}^{2}}=\frac{\cos ^{2} \theta}{n_{o}^{2}}+\frac{\sin ^{2} \theta}{n_{e}^{2}}$, and assuming $\theta$ is small, the phase shift can be written as

$$
\Delta \phi=\frac{2 \pi d}{\lambda} \frac{n_{o}\left(n_{o}^{2}-n_{e}^{2}\right)}{n_{e}^{2}} \theta^{2} .
$$

This expression, of course, only includes an incident skew angle in one plane; but a full description requires a contribution to the phase from a skew angle in an orthogonal plane, one where the ray's opposite component is refracted by a superposition of $n_{o}$ and $n_{e}$ instead of only $n_{o}$ (Figure 5.5). In this case, $n_{1}$ of Equation 5.14 becomes the refractive index composed of a superposition of $n_{o}$ and $n_{e}$ and $n_{2}$ is simply $n_{o}$. This arrangement reproduces Equation 5.15 with a minus sign allowing the complete phase shift (for angles of incidence $\theta$ in the horizontal plane and $\rho$ in the vertical) to be written

$$
\Delta \phi=\frac{2 \pi d}{\lambda} \frac{n_{o}\left(n_{o}^{2}-n_{e}^{2}\right)}{n_{e}^{2}}\left(\theta^{2}-\rho^{2}\right) .
$$

This expression now represents the total phase shift between any ray's orthogonal components of light at some nonzero angle with respect to the Pockels cell's optic axis. In considering the the voltage flip of a Pockels cell where one axis on the face of the cell labeled $n_{o}$ will flip between a refractive index of $n_{o} \pm \Delta n$, a substitution of this expression for $n_{o}$ in 


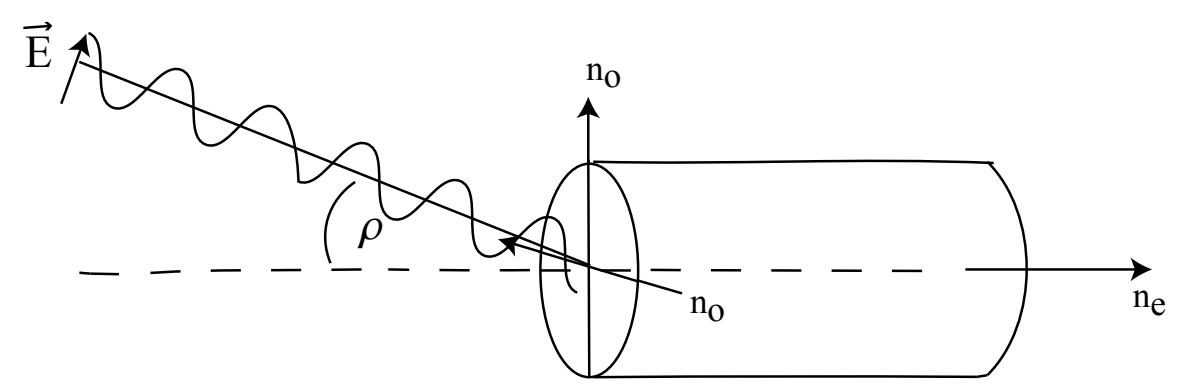

Figure 5.5: A ray incident at some angle $\rho$ with respect to the optic axis (labelled $n_{e}$ ) and in a plane orthogonal to that formed by the angle in Figure 5.4. This ray will see a superposition of indices of refraction $\left(n_{o}\right.$ and $\left.n_{e}\right)$ whereas a ray of normal incidence would see only $n_{o}$. A ray of orthogonal polarization to the one pictured would see only $n_{o}$ as well.

Equation 5.16 will yield (neglecting higher order terms in $\Delta n$ )

$$
\Delta \phi=\frac{2 \pi d}{\lambda}[\underbrace{\frac{n_{o}\left(n_{o}^{2}-n_{e}^{2}\right)}{n_{e}^{2}}\left(\theta^{2}-\rho^{2}\right)}_{\Delta} \pm \underbrace{\Delta n\left(3 n_{o}^{2}-n_{e}^{2}\right)\left(\theta^{2}-\rho^{2}\right)}_{\alpha}]
$$

where the antisymmetric $\Delta$ and symmetric $\alpha$ phases are labeled. Since only the $\Delta$ phase is relevant in discussing position differences arising from the skew effect, $\alpha$ will be neglected hereafter.

It should be mentioned that what we are applying here is a geometric optics analysis on a diffraction limited beam. This is a step forward in our understanding of the polarized source, and while quite instructive, is not rigorously justified. This caveat should be mentioned in case phase information can be incorporated into this model at some point in the future.

To integrate a Gaussian laser beam into this formalism, we must first consider the distribution of angles across the face of the beam. The evolution of the beam envelope where the angle of divergence changes within the Rayleigh range (defined as a distance $\pi \omega_{o}^{2} / \lambda$ away from the waist, the location where the spot size is smallest and divergence of the envelope 
is zero) means there must be some distribution of angles at each point across the face of the beam; at the waist, it was assumed this distribution at each point would be gaussian. The angles $\theta$ and $\rho$ in Equation 5.17 were changed to $\theta_{o}+\theta$ and $\rho_{o}+\rho$ where $\theta_{o}$ and $\rho_{o}$ indicate the angles between the optic axis of the Pockels cell and the center of the Gaussian beam and $\theta$ and $\rho$ indicate the angle of deviation of a particular ray from the center of the beam. $\theta$ and $\rho$ were then changed into expressions dependent upon a point of origin (at the waist) and a point where the beam is analyzed (some distance $l$ away from the beam waist). Figure 5.6 shows how such expressions were obtained.

To obtain an analytic expression for $\Delta x$, Equation 5.8 must be changed from a simple sum over all rays in a distribution to an integral over all rays originating from gaussian distributed angles at each point and a gaussian intensity across the beam face. The intensity of any laser beam over the $\mathrm{x}$ and $\mathrm{y}$ axes of its face at a distance $l$ from its waist before interacting with an analyzing power varies as

$$
I(x, y, l) \sim \frac{1}{w(l)^{2}} e^{-2\left(x^{2}+y^{2}\right) / w(l)^{2}}
$$

where $w(l)=w_{o} \sqrt{1+\left(l \lambda / \pi w_{o}^{2}\right)}$ is the radius of the beam spot encompassing $86 \%$ of the beam's intensity and $w_{o}$ is the beam's waist [72]. After interaction with an analyzing power, intensity depends upon the angles of incidence with the Pockels cell's optic axis and varies as

$$
I(x, y, l) \sim \frac{1}{w(l)^{2}} e^{-2\left(x^{2}+y^{2}\right) / w(l)^{2}} e^{-2\left(\theta^{2}+\rho^{2}\right)\left(\frac{\pi w_{o}}{\lambda}\right)^{2}}
$$

where the factor $\frac{\lambda}{\pi w_{o}}$ is the diffraction angle at which a gaussian beam will constantly diverge 


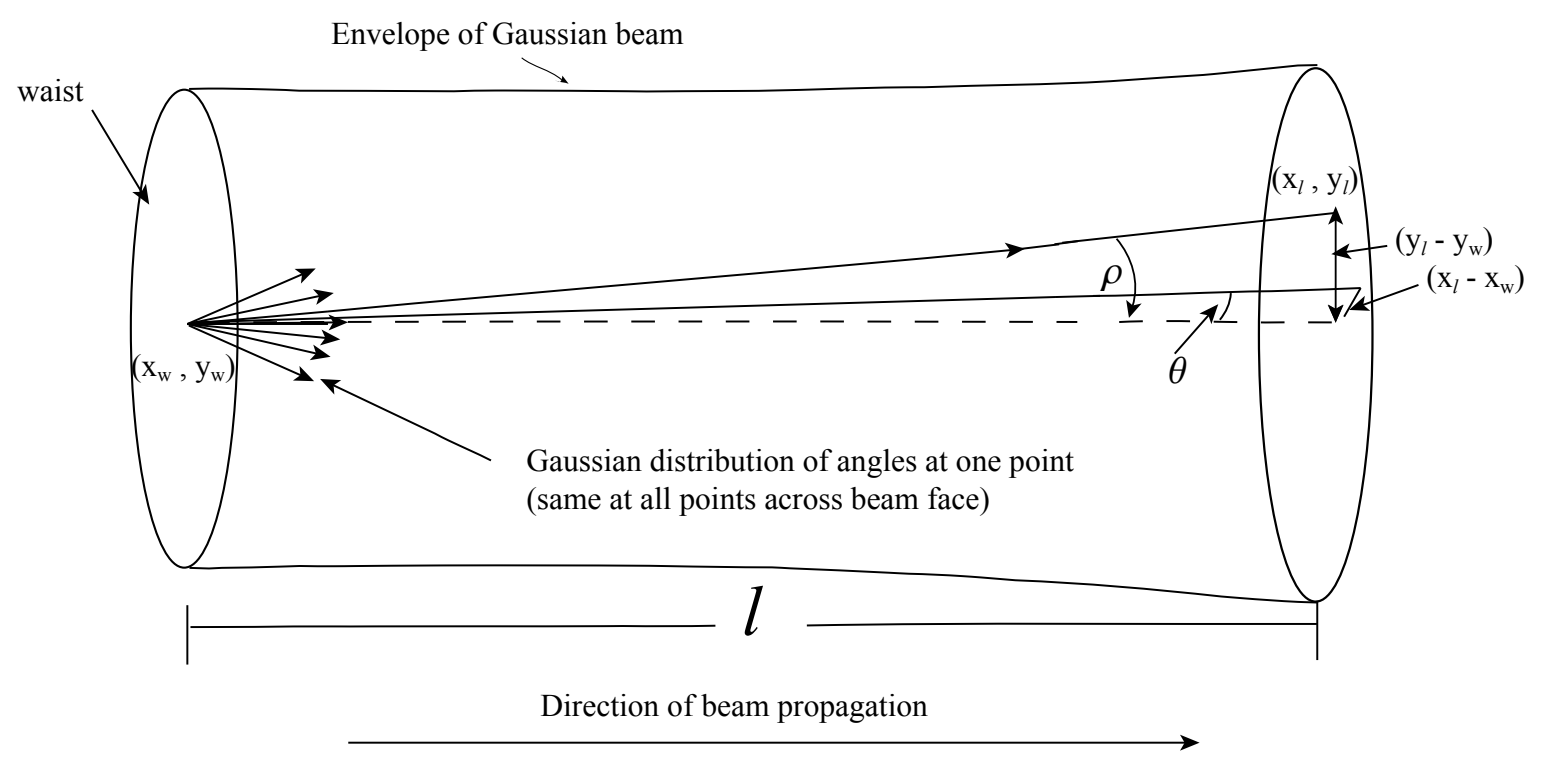

Figure 5.6: One ray from position $\left(x_{w}, y_{w}\right)$ is projected a distance $l$ to a position $\left(x_{l}, y_{l}\right)$ at an angle $\rho$ in the vertical plane and $\theta$ in the horizontal plane. Assuming small angles, $\rho=\left(y_{l}-y_{w}\right)$ and $\theta=\left(x_{l}-x_{w}\right)$. 
in the far-field (beyond the Rayleigh range)[76]. 86\% of all rays in a Gaussian distribution will travel at angles smaller than this with respect to the center of the beam; the ones at greater angles are not weighted heavily and it is assumed that they are not present in numbers significant enough to contribute. This may not be precisely the characteristics of the gaussian beam in our setup, but there's little reason to doubt that it is close.

The integral of our distribution can then be written

$$
\Delta\langle x\rangle=\frac{\int_{-\infty}^{\infty} \int_{-\infty}^{\infty} \int_{-\infty}^{\infty} \int_{-\infty}^{\infty} 2 A_{Q_{i}} e^{-2\left(x_{l}^{2}+y_{l}^{2}\right) / w(l)^{2}} e^{-2\left[\left(\theta_{o}-\theta\right)^{2}+\left(\rho_{o}-\rho\right)^{2}\right]\left(\frac{\pi w_{o}}{\lambda}\right)^{2}} x d x_{w} d y_{w} d x_{l} d y_{l}}{\int_{-\infty}^{\infty} \int_{-\infty}^{\infty} \int_{-\infty}^{\infty} \int_{-\infty}^{\infty} e^{-2\left(x_{l}^{2}+y_{l}^{2}\right) / w(l)^{2}} e^{-2\left[\left(\theta_{o}-\theta\right)^{2}+\left(\rho_{o}-\rho\right)^{2}\right]\left(\frac{\pi w_{o}}{\lambda}\right)^{2}} d x_{w} d y_{w} d x_{l} d y_{l}}
$$

where $\theta$ and $\rho$ can be written in terms of $x_{l}, x_{w}, y_{l}$ and $y_{w}$ as shown in Figure 5.6. Removing the " $x$ " in the numerator above leaves an expression for $\left\langle A_{Q}\right\rangle$ which, when calculated, leaves

$$
\left\langle A_{Q}\right\rangle=\frac{2 \pi d}{\lambda} \frac{n_{o}\left(n_{o}^{2}-n_{e}^{2}\right)}{n_{e}^{2}}\left(\theta_{o}^{2}-\rho_{o}^{2}\right),
$$

an expression for the integrated average of $A_{Q}$ which compares nicely with that of $\Delta \phi$ for a single ray in Equation 5.16 (shown to equal $A_{Q}$ ). This result gives us greater confidence in our model for the gaussian beam.

Evaluating Equation 5.20 results in the analytical form

$$
\Delta\langle x\rangle=\frac{\pi^{3} d w_{o}^{2}}{l \lambda^{3}}\left(\frac{n_{o}\left(n_{o}^{2}-n_{e}^{2}\right)}{n_{e}^{2}}\right) w(l)^{2}\left(\theta_{o}^{2}-\rho_{o}^{2}\right) \theta_{o}
$$

which reflects how position differences produced from the skew effect vary with the angles $\theta_{o}$ and $\rho_{o}$, as well as the distance $l$ between the beam's waist and the analyzing power; the 
laser beam's wavelength $\lambda$ was $800 \mathrm{~nm}$ and its waist $w_{o}$ was generally between $0.5-1 \mathrm{~mm}$.

To calculate $\Delta\left\langle x^{2}\right\rangle$, the difference in the expectation value of the squared average $x$ position of the laser beam, we replace the " $x$ " in Equation 5.20 with " $x$ ". The result after computing the integrals is

$$
\Delta\left\langle x^{2}\right\rangle=\frac{\pi d}{2 \lambda}\left(\frac{n_{o}\left(n_{o}^{2}-n_{e}^{2}\right)}{n_{e}^{2}}\right)\left(\frac{w_{o}^{4}}{l^{2} \lambda^{4}}\left(\pi w_{o}^{2}+l \lambda\right)^{2} \theta_{o}^{2}+\frac{\pi w_{o}^{2}+l \lambda}{\pi}\right)\left(\theta_{o}^{2}-\rho_{o}^{2}\right)
$$

after computation of the integrals, an expression for how spot size differences vary with parameters in the source setup.

\subsubsection{Steering}

Over the years, studies of beam $A_{\mathrm{LR}}$ 's have revealed position differences caused by the Pockels cell lensing or steering the beam in a helicity-correlated way. As mentioned earlier, polarization related effects can be studied by introducing a polarizer to provide an analyzer. Removing the downstream linear polarizer during a laser table study makes it possible to study effects that are not related to polarization. In such a case, position differences result from a helicity-correlated steering of the beam by the Pockels cell. This effect is not well understood, but is assumed to result from the different voltage applied to the cell from one helicity state to the next; this likely causes some change in the cell's shape or refractive index that results in a position difference. Placing one or two lenses downstream of the Pockels cell could change the effective lever arm and limit contributions from this effect, but at Jefferson Lab there is not enough room for them on the laser table in the injector tunnel. Fortunately, position differences due to steering remain small if the laser beam is 
transmitted through the Pockels cell center near its optic axis. There is still a remaining mystery with this effect in that a movement of the cell along the $\mathrm{x}$ axis results in a larger change in y position differences and vice versa for a movement along the y axis and position differences in $\mathrm{x}$; it seems the opposite result should be expected. This is not a crucial issue as it looks like $\Delta x$ and $\Delta y$ remain small if the beam travels near the center of the cell and can be canceled between slugs of data (a slug was a defined amount of data, usually 4 -8 CODA runs lasting about an hour a piece) by the insertion of the half-wave plate, but it remains an interesting conundrum.

\subsubsection{Charge Asymmetry Structure}

When a Pockels cell flips to a different helicity, it takes some time for the cell to settle into a mode where it is producing optimal circularly-polarized light, and a cell's previous helicity windows determine how long this settling takes. When high voltage is applied to the Pockels cell, there is a time constant associated with the phase retardation that it applies to laser light. Helicity pairs with different previous states were measured with different timeaveraged charge asymmetries that resulted in a multipeak structure to the charge asymmetry (Figure 5.7). This settling time and how it varied is apparent for the four patterns in Figure 5.8.

The red structure in Figure 5.7 resulted from running in mode referred to as "quadrandom" where the helicity state for the fourth window is pseudorandomly selected; only 2 possibilities ( $L R R L$ and $R L L R)$ exist but that allows for 4 different patterns on the Pockels cell's HV switch $(R R L, L R L, R L R$, and $L L R)[49]$. A helicity mode that simply toggles $(R L R L R L)$ has only one helicity pattern and would result in a structure with only one 


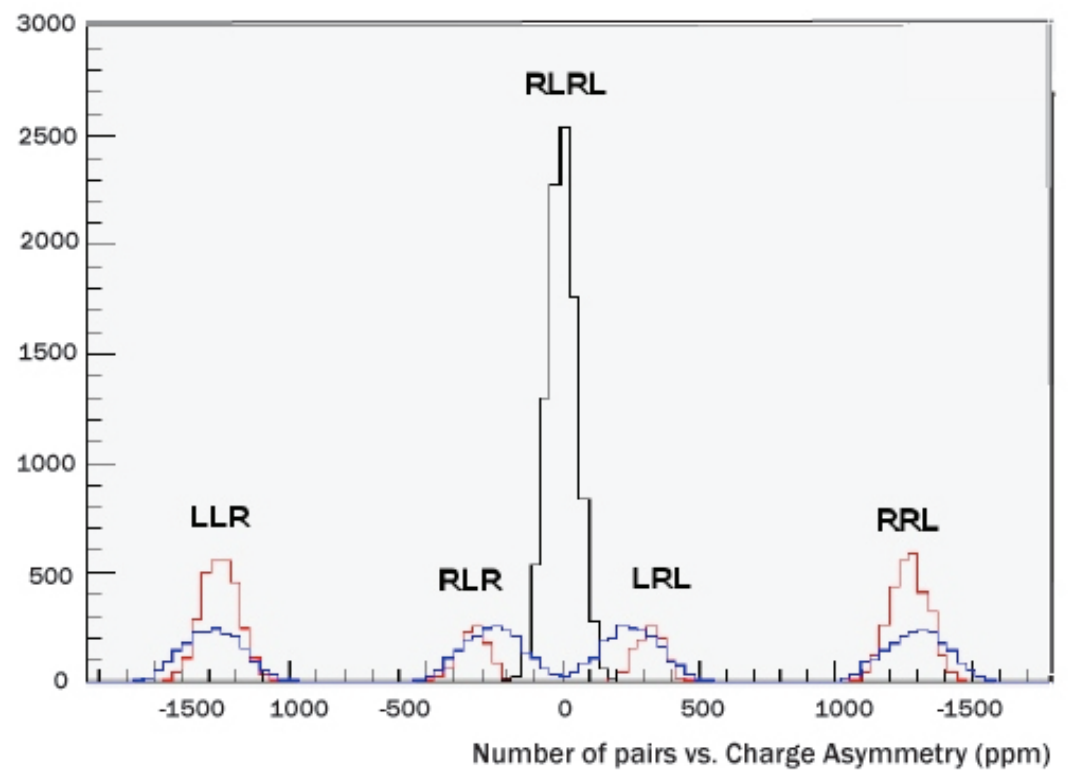

Figure 5.7: Charge asymmetry structure for toggle (black), quad-random (red), and pair-random (blue) helicity modes at the Pockels cell's HV switch. The number of pairs from each pattern vs. $A_{Q}(\mathrm{ppm})$ are displayed above.

peak. The data for Figure 5.8 were accumulated by measuring the charge asymmetry at multiple points within a helicity window (oversampling).

It was also discovered that not only does the settling time depend on a pair's previous helicity state, but also on the state previous to that one. In pair-random mode, the first state of each pair is pseudorandomly selected; this allows for more helicity patterns at the HV switch and as a consequence, broadens the peaks of the charge asymmetry structure (blue data in Figure 5.7). Fortunately, each peak in the structure has a similarly populated complement with an equal but opposite charge asymmetry; this helps to cancel any beam asymmetries that might result due to a single peak in the structure. 


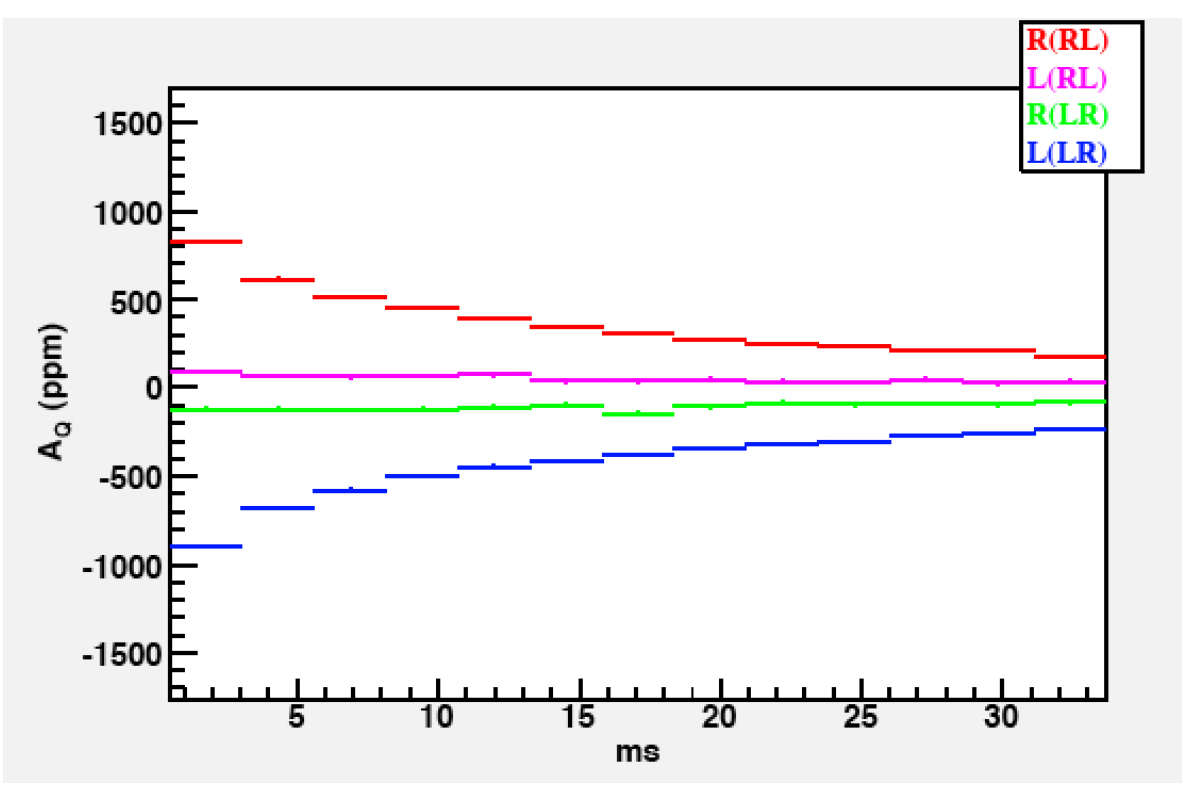

Figure 5.8: The charge asymmetry (ppm) vs. elapsed time (ms) over a helicity window. The four different helicity patterns reaching the HV switch in quad-random mode are labeled as indicated in the upper right.

Studies were also conducted to rule out the possibility of the high-voltage (HV) switch or capacitance from the cables connecting the $\mathrm{HV}$ to the $\mathrm{PC}$ as a source of the history effect [49]. It was concluded that the multipeak structure was due to the intrinsic settling time of the Pockels cell material. Since the integration of a helicity window's detector flux did not begin until $700 \mu$ s after the beginning of the helicity window, this peak structure was not an issue during HAPPEX-II.

\subsubsection{Vacuum Window and Rotatable Half-Wave Plate}

Among the downstream optics contributing to the phase shift $\Delta^{o}$ in Equation 5.5 are the vacuum window and the rotatable half-wave plate (RHWP). As we consider how to suppress beam $A_{\mathrm{LR}}$ 's, it is useful to separate out of the offset term $\Delta^{o}$ contributions that arise from the rotatable half-wave plate and the vacuum window The vacuum window is the last element in 
the source beamline and has a significant stress-induced birefringence. For the remainder of the chapter, we redefine $\Delta^{o}$ to exclude the residual birfringence associated with the vacuum window and the RHWP. The vacuum window can be represented as

$$
J_{V W}=\left[\begin{array}{cc}
\cos ^{2} \rho+e^{i \beta} \sin ^{2} \rho & \left(1-e^{i \beta}\right) \sin \rho \cos \rho \\
\left(1 e^{i \rho}\right) \sin \rho \cos \rho & \sin ^{2} \rho+e^{i \beta} \cos ^{2} \rho
\end{array}\right]
$$

where $\beta$ and $\rho$ are, respectively, the phase retardation and orientation angle of the vacuum window.

Recounting from Section 5.2.1 the asymmetric transport element with transmission coefficients $T_{x^{\prime}}$ and $T_{y^{\prime}}$ along some axes $x^{\prime}$ and $y^{\prime}$, it can be represented as

$$
J_{A T}=\left[\begin{array}{cc}
T+\frac{\varepsilon}{2} \cos 2 \theta & \frac{\varepsilon}{2} \sin 2 \theta \\
\frac{\varepsilon}{2} \sin 2 \theta & T-\frac{\varepsilon}{2} \cos 2 \theta
\end{array}\right]
$$

where $T=\left(T_{x^{\prime}}+T_{y^{\prime}}\right) / 2, \varepsilon=T_{x^{\prime}}-T_{y^{\prime}}$, and $\theta$ is the angle between $x^{\prime}$ and the horizontal axis. The asymmetric transport element (i.e., photocathode), as has been previously discussed, provides an analyzing power that is sensitive to the orientation of the of the linear polarization. The final electric field vector upon the addition of the vacuum window becomes

$$
\vec{E}_{f}=J_{A T} \cdot J_{V W} \cdot J_{P C} \cdot \vec{E}_{i}
$$

Having separated out the vacuum window from $\Delta^{o}$, it contributes as an additional term in 
the asymmetry equation:

$$
A_{Q}=-\frac{\varepsilon}{T}\left[\left(\Delta-\Delta^{o}\right) \cos 2 \theta+\beta \sin (2 \theta-2 \rho)\right]
$$

Next we consider the rotatable half-wave plate (RHWP) which sits right before the vacuum window and is used to rotate the laser beam's residual linear polarization to minimize contributions to beam asymmetries that arise from interactions with the cathode's analyzing power. The RHWP is allowed an arbitrary orientation $\psi$ and a deviation $\gamma$ from perfect half-wave retardation and can be represented as

$$
J_{R H W P}=\left[\begin{array}{cc}
\cos ^{2} \psi+e^{i(\pi+\gamma)} \sin ^{2} \psi & \left(1-e^{i(\pi+\gamma)}\right) \sin \psi \cos \psi \\
\left(1 e^{i(\pi+\gamma)}\right) \sin \psi \cos \psi & \sin ^{2} \psi+e^{i(\pi+\gamma)} \cos ^{2} \psi
\end{array}\right]
$$

where $\psi$ and $\gamma$ are, respectively, the angle of orientation and deviation from half-wave retardation of the RHWP. The final electric vector here is calculated as

$$
\vec{E}_{f}=J_{A T} \cdot J_{V W} \cdot J_{R H W P} \cdot J_{P C} \cdot \vec{E}_{i}
$$

and the resulting intensity asymmetry

$$
A_{Q}=\frac{\varepsilon}{T}\left[\left(\Delta-\Delta^{o}\right) \cos (2 \theta-4 \psi)-\gamma \sin (2 \theta-2 \psi)-\beta \sin (2 \theta-2 \rho)\right]
$$

A discussion of how the RHWP is used to minimize beam $A_{\mathrm{LR}}$ 's and how to cope with the problems related the vacuum window will appear in Section 5.4 


\subsubsection{QE Anisotropy Gradients}

It is reasonable to consider whether spatial variations in the cathode's analyzing power $(\varepsilon / T$ in Equation 5.5) or its orientation $\theta$ are responsible for any helicity-correlated asymmetries on the electron beam. It is assumed that any $\Delta$-type phase shift in the laser light interacting with such a gradient at the cathode results in a helicity-correlated position difference. This makes minimizing $\Delta$ crucial as the cathode's gradient is not possible to suppress; the cathode's orientation $\theta$ can only be rotated with respect to the laser beam's residual linear polarization with the RHWP. The method for doing so will be discussed in section 5.5.

\subsubsection{Cross-talk (Electronic and otherwise)}

It was discovered during the He measurement that the helicity signal which triggers voltage changes on the Pockels cell was not electrically isolated. This signal leakage was significant enough to induce large position differences in the $100 \mathrm{KeV}$ injector. All other sources of position differences were dominated by this effect for the He measurement. Fortunately the cancellation was sufficient and other systematic errors dominated, but an important lesson was learned in taking great care to isolate the helicity signal in the future [77].

The three independent beams running to the three halls at Jefferson Lab also experience cross-talk between their helicity-correlated properties; the cause of this at the photocathode is not well understood, but has proven to be significant [77]. 


\subsection{Minimization of beam $A_{\mathrm{LR}}$ 's}

The polarized source for HAPPEX-H performed better than any previous setup, and a number of strategies were employed to achieve our outstanding results at minimizing beam $A_{\mathrm{LR}}$ 's These included careful selection of the Pockels cell, minimization of the laser skew effect with scans in pitch and yaw, selection of a RHWP angle to limit asymmetries caused from the interaction of the cathode's analyzing power with the residual linear polarization of the

laser beam, slow reversals of the beam $A_{\mathrm{LR}}$ 's with respect to the physics asymmetry to achieve cancellations, and control of $A_{Q}$ through use of various feedback systems. In what follows, these methods will be discussed in more depth as well as other more minor minimization techniques.

\subsubsection{Pockels cell selection}

The selection of which Pockels cell to use is largely determined by the spatial variation of the residual birefringence across the cell. The birefringence in the cell can be caused by stress induced from growing the crystal, cutting the crystal, and mounting the crystal in its housing. The less variation measured near the center, the smaller the position differences due to birefringence gradients, and hence, a better cell to use during the experiment run. After aligning the cell as described in Section 5.1.2 with a cleanup polarizer upstream of the Pockels cell, a linear polarizer was placed downstream of the cell and a quad photodiode downstream of that (Figure 5.9).

The linear polarizer magnifies the analyzing power by about a factor of 30 relative to the photocathode and therefore provides increased sensitiity to the birefringence gradients 


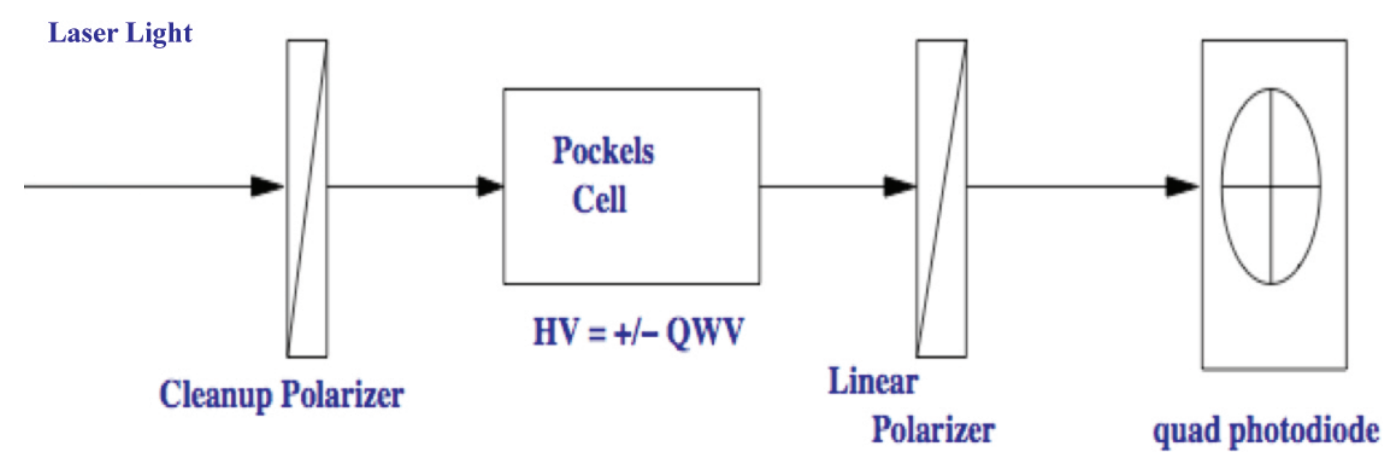

Figure 5.9: The setup used to measure position differences due to birefringence gradients (QWV $=$ quarter-wave voltage).

within the cell. The quad photodiode measures the $x$ and $y$ position differences, $\Delta x$ and $\Delta y$, and the charge asymmetry, $A_{Q}$.

By translating the Pockels cell across the laser beam spot, the variation in residual birefringence can be measured across both the $x$ and $y$ dimensions. The setup displayed in Figure 5.9 was used for this measurement. The Pockels cell is fastened into a mount and this mount is attached to a translation stage where micrometer dials are rotated to move the cell in both the $x$ and $y$ directions.

Figure 5.10 displays $\Delta x, \Delta y$, and $A_{Q}$ for one of the many Pockels cells ("Sam") characterized on the laser table. Over the range of "Sam"'s horizontal dimension, $\Delta x$ varies from 1 to $-2 \mu \mathrm{m}$, but is small near the cell's center (around 11-12 $\mathrm{mm}$ on the horizontal axis in Figure 5.10) where the laser beam will travel. "Sam"'s variation in birefringence here was the slightest of all characterized cells and was therefore chosen as the one to run with during HAPPEX-He and -H.

When the linear polarizer downstream of the Pockels cell in Figure 5.9 is removed, similar $x$ and $y$ translation scans can be performed to measure how steering effects on the beam vary 

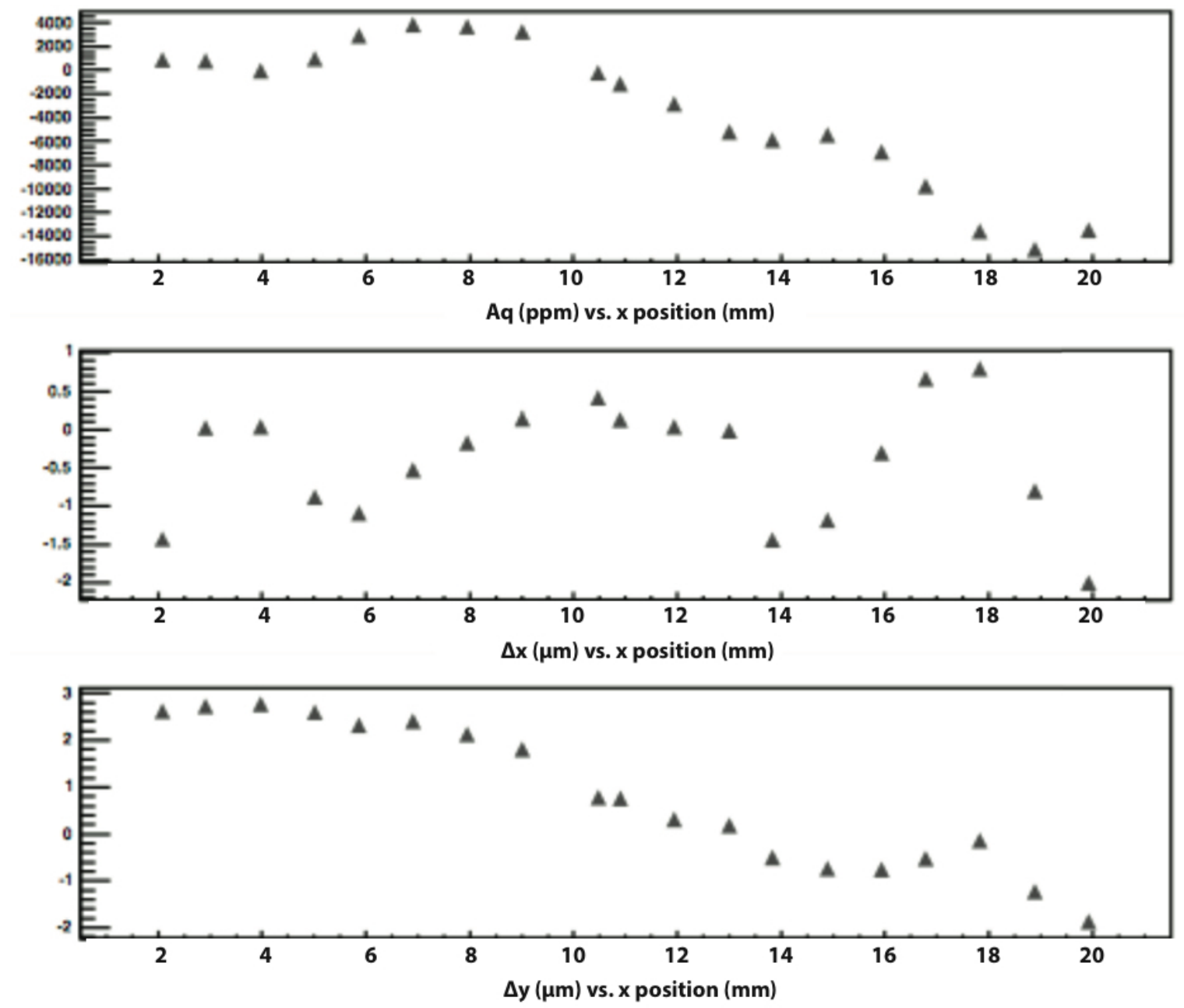

Figure 5.10: On top: $A_{Q}(\mathrm{ppm})$ vs. $\mathrm{x}(\mu \mathrm{m})$, in the middle: $\Delta x$ vs. $\mathrm{x}(\mu \mathrm{m})$, and on the bottom: $\Delta y$ vs. $\mathrm{x}(\mu \mathrm{m})$ due to birefringence gradients across the horizontal dimension of the Pockels cell. 
across the Pockels cell face. Removing the downstream analyzer removes any sensitivity to polarization effects. Only a helicity-correlated steering or "lensing" of the beam is measured. This effect is most often minimized when laser light travels through the cell closest to its geometric center.

\subsubsection{Minimizing the Laser Skew Effect}

With the discovery that the laser light's slight angle of incidence with the Pockels cell's optic axis was responsible for large position differences, a method for limiting this angle was needed in our setup. This effect presented itself as a large offset in the measurement of position differences as a function of position across the Pockels cell face, and dominated all other features of the data (Figure 5.11). We discovered that this offset did not change until we adjusted the pitch and yaw screws on the Pockels cell mount, equivalent to changing the angle made with the optic axis of the Pockels cell in either the horizontal or vertical plane. Measuring the sensitivity to changes in pitch and yaw provided the adjustment needed to minimize position differences due to any phase gradients in the beam from birefringence gradients in the Pockels cell, skew angle, or optics upstream thereof.

Figure 5.12 shows the source's sensitivity to the horizontal skew effect as measured from the setup that appears in Figure 5.9. The screw on the Pockels cell mount adjusting the yaw position was moved in increments of $\frac{1}{2}$ turn and data were collected over a range of \pm 2.5 turns. As displayed in the slopes of the fits to the data next to the plots, $\Delta x$ varied -24.09 $\mu \mathrm{m}$ per turn whereas $\Delta y$ varied $-5.76 \mu \mathrm{m}$ per turn. At 8 milliradians per turn on the screws, these numbers scale up by a factor of 8 to give the sensitivity per milliradian of incident skew angle. This scan only measures the source's sensitivity to the horizontal angle, or yaw; 

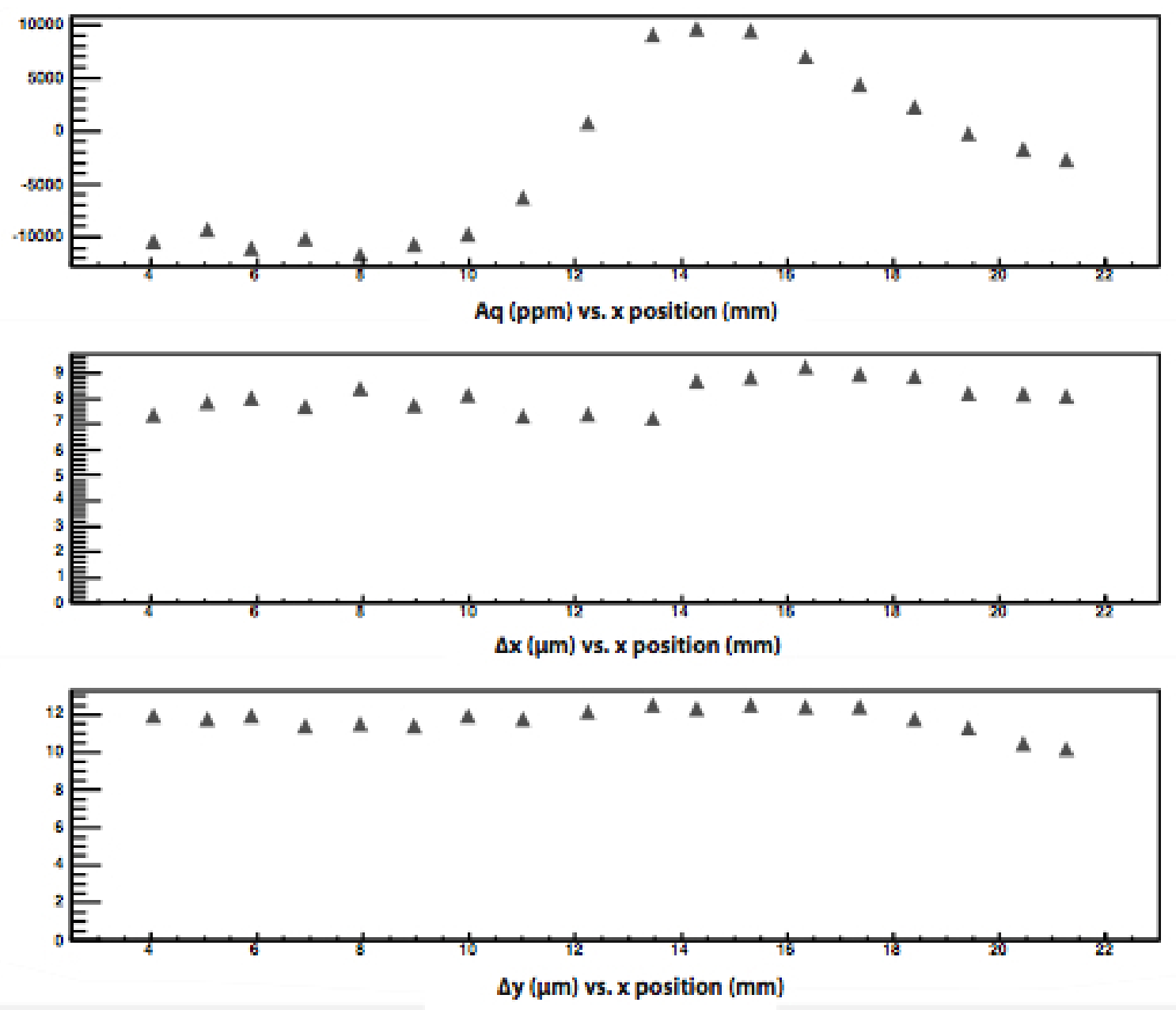

Figure 5.11: The same measurements here as displayed in Figure 5.10. The offset in the data for $\Delta x$ around $8 \mu \mathrm{m}$ and $12 \mu \mathrm{m}$ for $\Delta y$ is due to a dominating phase gradient produced from a small angle of incidence of the laser light with the optic axis of the Pockels cell. 
a measure of sensitivity to the vertical angle is taken by scanning through a range of angles with the pitch adjustment screw. Due to a small sensitivity in the orthogonal dimension (y in the case of Figure 5.12), attempts at minimizing position differences in one dimension affect minimization attempts in the other. In this case, linear algebra must be performed with the measured pitch and yaw sensitivities to obtain the position on both screws needed to best minimize $\Delta x$ and $\Delta y$.

The results after a pitch and yaw adjustment are shown in Figure 5.13 where $\Delta x$ at the Pockels cell's center $(\sim 12 \mathrm{~mm}$ on the $\mathrm{x}$ axis $)$ is zero. $\Delta y$ is a bit larger $(\sim 1.5 \mu \mathrm{m})$ but when considering the difference of an approximate factor of 30 between the analyzing power of our linear polarizer and the cathode, the differences off the cathode would be only around $50 \mathrm{~nm}$. Further minimization would be expected by the adiabatic damping of the accelerator's optics.

\subsection{Controlling the PITA Effect}

As discussed in section 5.2.1, the interaction of the laser beam's residual linear polarization with the photocathode's analyzing power creates a charge asymmetry, $A_{Q}$. This is known as the PITA effect and can be minimized by applying an offset to the Pockels cell voltages to reduce the component of linear polarization along the cathode's analyzing power axis. This offsetting voltage is determined from the PITA slope, the measurement of $A_{Q}$ vs. voltage discussed in section 5.2.2.

The rotatable half-wave plate (RHWP) can be used independently downstream of the Pockels cell (Figure 5.14) to control $A_{Q}$ by rotating the laser beam's residual linear polar- 


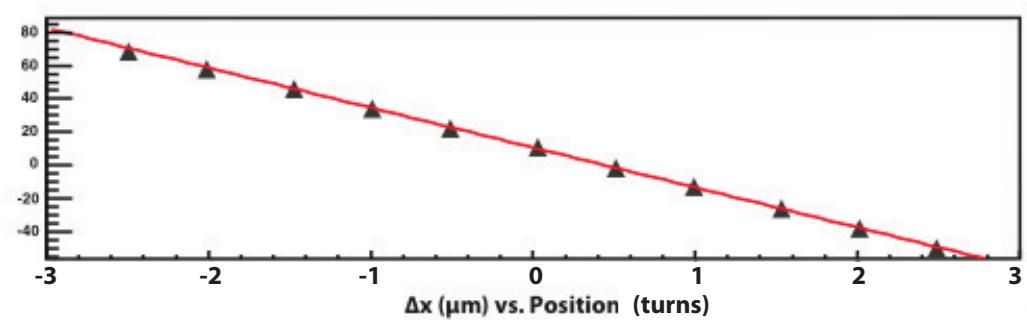

\section{$\mathrm{Dx}=10.50+$ $-24.09 * \mathbf{x}$}

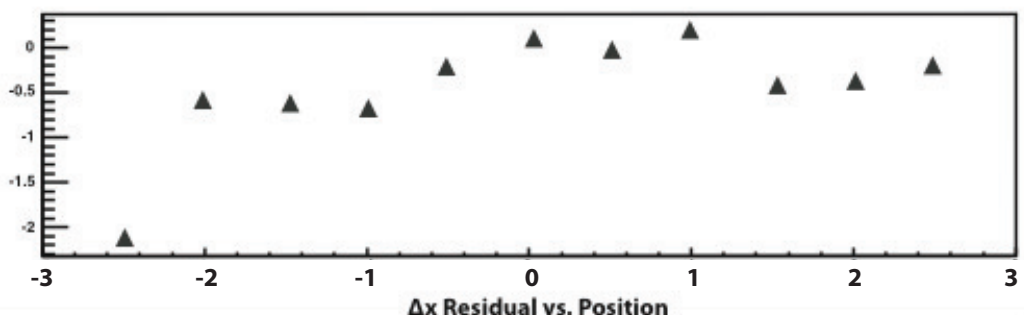

$$
\mathrm{Dx}=0 @ \mathrm{x}=0.4
$$

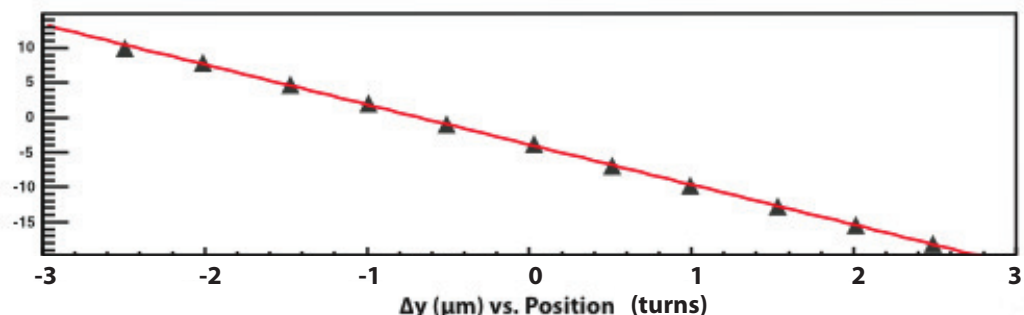

$$
\begin{gathered}
\text { Dy }=-3.91+ \\
-5.76 * x
\end{gathered}
$$

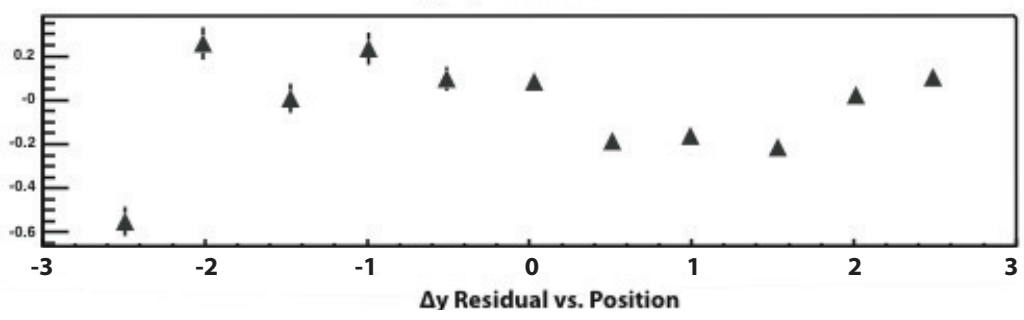

$$
\text { Dy }=0 \text { 0 } \mathrm{x}=-\mathbf{0 . 7}
$$

Figure 5.12: A measure of the source's sensitivity to the yaw adjustment using the setup in Figure 5.9. The top two plots are $\Delta x(\mu \mathrm{m})$ vs. turns in yaw and the residual, respectively; the bottom two are the same for y. The linear fit to the data is displayed on the right and the number of turns needed to zero $\Delta x$ and $\Delta y$ are found in the slopes of these fits. 

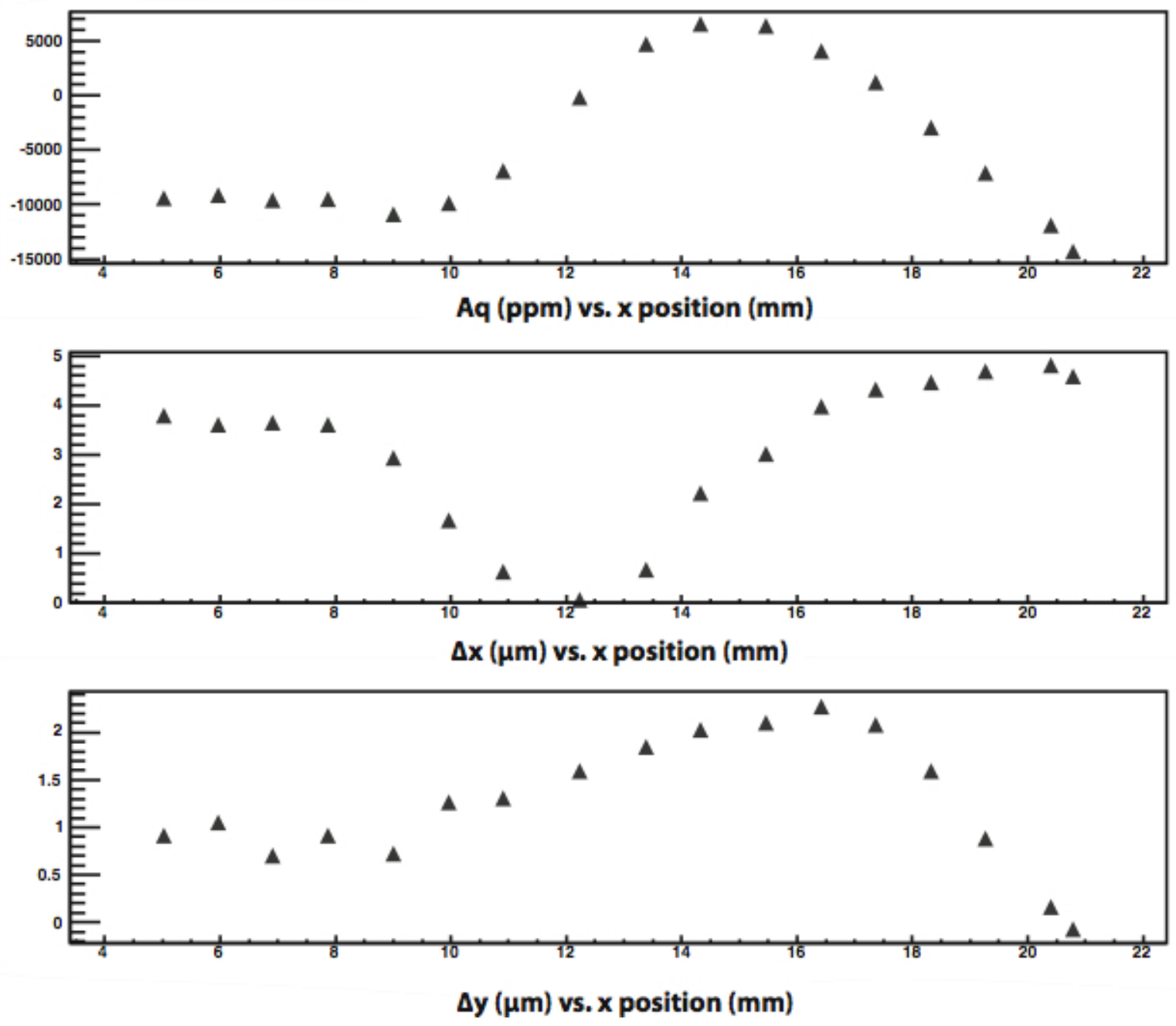

Figure 5.13: Same measurement as in Figure 5.11 after adjustments to pitch and yaw on Pockels cell mount. 


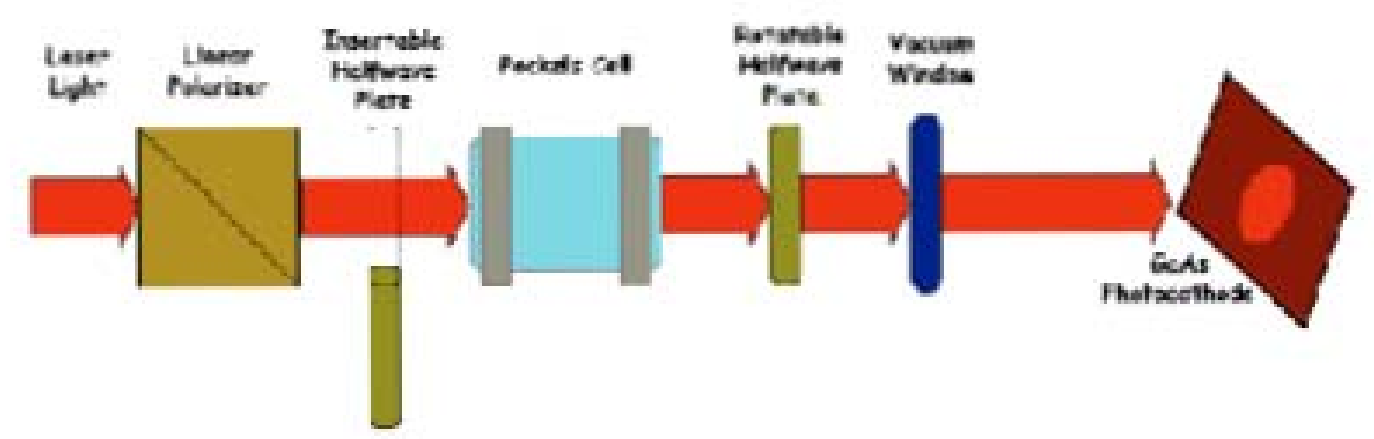

Figure 5.14: A schematic diagram of the optics used to produce the circularly polarized light and control beam $A_{\mathrm{LR}}$ 's.

ization $45^{\circ}$ with respect to the cathode's analyzing power axis (Figure 5.15); notice how the analyzing power axis represented by the black arrow in Figure $5.15 \mathrm{~b}$ is not aligned along the residual linear polarization of either helicity state when the light is rotated. Some sensitivity to the cathode's analyzing power should remain, however, allowing the PITA offset voltage to cancel the linear polarization induced by other optical elements, most specifically the vacuum window. While reducing the sensitivity but keeping it nonzero, it is possible to reduce position differences due to Pockels cell phase gradients while allowing a large enough range of PITA voltages to tune out the average residual linear polarization from the vacuum window. This gives us two knobs of control over the effects of phase gradients from two different sources. Limiting too far our sensitivity to the cathode's analyzing power would prevent the needed suppression of some vacuum window effects from the cathode's gradient in its analyzing power. The vacuum window's birefringence, however, cannot be suppressed.

The data gained from rotating the RHWP to find the most desirable angle for the experiment run can be fit to an expression of the form

$$
\Delta x=c+a \sin \left(2 \theta+\phi_{1}\right)+b \sin \left(4 \theta+\phi_{2}\right)
$$



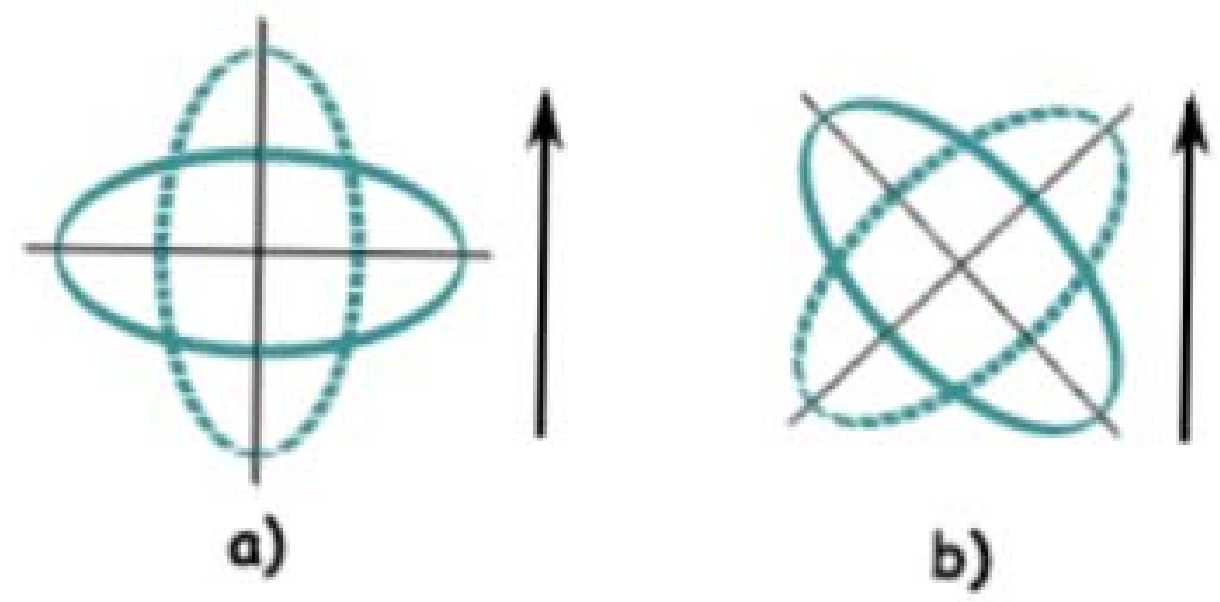

Figure 5.15: (a) This orientation with the residual linear polarization aligned along the photocathode's analyzing power axis maximizes $A_{Q}$ (b) at an orientation of $45^{\circ}, A_{Q}$ is minimized.

where $c$ is an offset usually dominated by the effects of steering but includes also those from the vacuum window, $\theta$ is the RHWP's angle of orientation with respect to some arbitrary axis, and $\phi_{1}$ and $\phi_{2}$ are phases having to do with the RHWP's angle of orientation to the analyzing power. The magnitude of the $4 \theta$ term in Equation 5.31 indicates the size of Pockels cell birefringence, cathode analyzing power gradients, or other gradients upstream of the Pockels cell. The $2 \theta$ term represents the size of some deviation from half-wave retardation of the RHWP. The magnitude of these terms and whether they flip sign with the insertion of the insertable half-wave plate (IHWP) reveal more information about the sources of helicitycorrelated beam asymmetries and whether they will cancel with IHWP slow reversal. The IHWP will be discussed next, and the various features in the data obtained from scanning the RHWP angles will be discussed in Section 5.5.

It should be mentioned again that the birefringence gradient in the vacuum window is the one source that could not be reduced. A new polarized gun will be installed at Jefferson 
Lab that will permit the rotation of the photocathode; this will rotate the analyzing power and allow for reduction of vacuum window effects, the last known uncontrollable source of PITA-induced position differences.

\subsubsection{Helicity Slow Reversal}

Further minimization of beam $A_{\mathrm{LR}}$ 's was achieved through slow reversal of the electron beam helicity's sign relative to that of all electronic helicity signals. This was accomplished by placing an insertable half-wave plate (IHWP) just upstream of the Pockels cell on the source table to rotate the incoming linear polarization by $90^{\circ}$ (Figure 5.14). In doing this, the phase shift induced by the Pockels cell would change sign, and with it, the helicity of the beam.

This method allowed for the cancellation of any beam $A_{\mathrm{LR}}$ 's dependent upon the electronic helicity signal and not the beam polarization.

However, many PITA-type ${ }^{\text {beam }} A_{\mathrm{LR}}$ 's will not cancel with the insertion of the IHWP; they will change sign along with the beam polarization. The residual linear polarization coupling to the QE anisotropy will not cancel as the orientation of the linear polarization will simply rotate $90^{\circ}$ and hence, change sign along with the helicity of the beam. The QE anisotropy gradient itself (without the residual linear polarization coupling) also will change sign, and hence, will not cancel. It is possible, however, to gain some measure of cancellation of position differences one could arrange for a much larger PITA slope in one helicity state compared to the other. The RHWP could then be oriented to preserve the large PITA slope and perhaps achieve a reasonable cancellation of effects arising from the upstream optics [74]. Steering, since it depends on only the Pockels cell voltage and not the helicity of the beam, will cancel upon IHWP insertion. 


\subsubsection{Feedback}

The minimization techniques discussed thus far are standard for comparatively small beam $A_{\mathrm{LR}}$ 's. However, other methods are necessary if some beam $A_{\mathrm{LR}}$ 's grow in size and need correction during the experimental run. For example, $A_{Q}$ could become large thereby implying a large $\Delta$ phase shift that leads to large position differences upon interacting with an analyzing power gradient at the cathode. A correction here is necessary to avoid the accumulation of large systematic errors. $A_{Q}$ itself, without large affects on position differences, could become large and need corrections with feedback as well.

\section{PITA Feedback}

The dominant method of feedback employed during the 2005 running of HAPPEX was the adjustment every 2 minutes of the Pockels cell voltages to zero $A_{Q}$. Since the PITA slope is a measure of the change in $A_{Q}$ for every volt of adjustment, this slope was used to calculate what change in the Pockels cell voltage was necessary to zero $A_{Q}$ averaged over a two minute interval. It is important to investigate whether corrections to an increasing beam $A_{\mathrm{LR}}$ is real and not masking a problem better addressed by some method other than feedback. For example, clipping of the beam in the injector could induce a large $A_{Q}$ that is best fixed by an adjustment to the accelerator's optics, and feedback should not be used to fix a polarized source that has not been set up to minimize ${ }^{\text {beam }} A_{\mathrm{LR}}$ 's to the optimum extent possible. To do so would only make it possible for higher-order effects to cause systematic problems and go unnoticed. 


\section{Hall C IA Feedback}

At some point during the the HAPPEX run, it was discovered that a large $A_{Q}$ on the Hall C beam would induce the same on Hall A's beam. This "crosstalk" between the two halls is not well understood, but may be related to the Pockels cell being optimized for only the Hall A beam while a different laser wavelength was used for the Hall $\mathrm{C}$ laser resulting in uncompensated helicity correlated effects at the cathode that could manifest themselves on the Hall A beam.

The Hall C "IA cell" was used to keep its $A_{Q}$ below 100 ppm. An IA cell system consists of a Pockels cell between two linear polarizers that can work as a variable electro-optic shutter [77]. In five minute intervals, components of linear polarization are added to or taken away from the Hall $\mathrm{C}$ laser beam by adjusting the voltage on the IA cell to minimize Hall C's $A_{Q}$, and correspondingly, its effects on the Hall A beam.

\subsubsection{Adiabatic Damping}

If the matching between sections of the accelerator are free of $X Y$ coupling, the position differences can be damped by $\sqrt{p / p_{o}}$ as the beam is accelerated from momentum $p_{o}$ to $p$. This effect is due to the adiabatic damping of the $x / x^{\prime}$ phase space for a beam under acceleration [78]. The beam emittance varies inversely with the beam momentum and is defined as the invariant phase space area based on the beam density matrix. The beam's projected size and divergence, and hence the difference orbit amplitude (the size of the deviation from the nominally correct orbit), are proportional to the square root of the emittance multiplied by the beta function. In the ideal case from the $100 \mathrm{keV}$ injector $(p$ 
$=355 \mathrm{keV}$ ) to $3.3 \mathrm{GeV}$ in the injector halls at Jefferson Lab, position differences can be reduced by a factor of $\sqrt{3 \mathrm{GeV} / 355 \mathrm{keV}} \sim 95$.

In practice, this ideal theoretical case is hard to achieve. As an electron beam is transported, correlations between $x, x^{\prime}, y$, and $y^{\prime}$ develop making the phase space a rotated ellipsoid. While the total phase space remains small, its projection into any coordinate can be large allowing helicity-correlated changes in position and angle to also be large. [77].

Imperfections in the design of the accelerator optics at the $10^{-3}$ level can lead to large couplings in position and angle or growth in one dimension of the phase space. The coupling originates from large oscillations around the central trajectory of the beam, exposing it to imperfections in magnetic or RF acceleration elements [77]. The best method for minimizing this coupling is to tune the accelerator optics back to the original machine design, a process called "matching" and referred to in the opening sentence of this section.

During the 2005 HAPPEX-II hydrogen run, estimates of adiabatic damping were complicated by the small size of the beam $A_{\mathrm{LR}}$ 's, but the reduction in position differences was measured to be at least a factor of 5; some estimates were as large as 30 [77].

\section{$5.5 \quad 2005{ }^{4} \mathrm{He}$ and H Source Setups}

This section will describe some of the challenges we encountered with the source during its setup and running of the experiment. The hydrogen section will also discuss more features of the RHWP scans from the 2005 HAPPEX-H data and how they are used to help identify

problems. The average beam $A_{\mathrm{LR}}$ 's from both the helium and hydrogen runs are presented in Table 5.1. 


\begin{tabular}{|c|rl|rl|}
\hline & \multicolumn{2}{|c|}{ HAPPEX-He } & \multicolumn{2}{|c|}{ HAPPEX-H } \\
\hline$A_{Q}$ & -0.38 & $\mathrm{ppm}$ & 0.41 & $\mathrm{ppm}$ \\
\hline$A_{E}$ & 3 & $\mathrm{ppb}$ & 0.2 & $\mathrm{ppb}$ \\
\hline$\Delta x$ & -0.2 & $\mathrm{~nm}$ & 0.5 & $\mathrm{~nm}$ \\
\hline$\Delta x^{\prime}$ & 4.4 & $\mathrm{nrad}$ & -0.2 & $\mathrm{nrad}$ \\
\hline$\Delta y$ & -26 & $\mathrm{~nm}$ & 1.7 & $\mathrm{~nm}$ \\
\hline$\Delta y^{\prime}$ & -4.4 & $\mathrm{nrad}$ & 0.2 & $\mathrm{nrad}$ \\
\hline
\end{tabular}

Table 5.1: Average beam asymmetries in intensity, energy, and position and angle differences in the $\mathrm{x}$ and $\mathrm{y}$ dimensions for the 2005 HAPPEX-II runs. This data includes the polarization reversal with the IHWP.

\subsection{1 $2005{ }^{4}$ He Tunnel Setup}

The source's performance during the helium run was dominated by trying to understand the source of large position differences displayed in Figure 5.16, most prominently for $x$ at bpm4a. Before the experimental run, an enclosure was built for the polarized source to house the lasers, optics, and computers. Mistakenly, a common electronic ground was not created after the completion of this project and some leakage of the helicity signal created ground loops and as a consequence, caused the large helicity-correlated beam deflections evident in Figure 5.16. For $\Delta x$ at bpm4a in Figure 5.16, the 2-6 $\mu \mathrm{m}$ position differences were a major puzzle that remained unresolved after many diagnostic tests. Once the Pockels cell was switched off and the position differences remained this large, possible reasons for this observation centered on the presence of ground loops in the electronics. Large position differences, albeit an order of magnitude less than for $x$, appear at the same bpm for $\mathrm{y}$, and they became large for $x$ at bpm4b once the voltage leads on the Pockels cell were switched around slug 40 (i.e., polarity reversed). Table 5.1 show the large discrepancy in angle position differences between the $\mathrm{He}$ and $\mathrm{H}$ runs due the presence of these ground loops. After identifying this as the cause, a tunnel access was made to create a common electronic 
ground, and the data shows remarkable improvements after slug 78.

Figure 5.16 also displays an aspect of the source for which we were fortunate while trying to identify this problem: cancellation between IHWP "IN" and "OUT" states. A minus sign was placed by hand in front of the position difference data for the IHWP "IN" state to indicate whether we could expect any cancellation between the IHWP states while accumulating data. Again, this is further evidence of beam deflection to one side or the other based on the sign of the helicity signal in the electronics. We were also fortunate this happened during the He run as the expected physics asymmetry is roughly 8 ppm and thus is less sensitivity to beam $A_{\mathrm{LR}}$ 's than is the $\mathrm{H}$ measurement (a measurement of $\sim 1.6 \mathrm{ppm}$ ). It was a valuable lesson learned in a fortuitous way.

When setting up the source for the HAPPEX- He run, we were cautiously optimistic upon finishing the setup in the injector tunnel, but of course, we were not able to see how well we had done until we rid the source of electronic ground loops later in the run. Due to space constraints, we had to set up our quad photodiode above the laser table with the quad photo crystal pointing downward parallel to the table. The most troubling part of the setup was a position difference effect that was polarization dependent (changes sign upon IHWP insertion), but was observed in the absence of an analyzing power. We have no formalism for understanding how this could happen, and this may have been a consequence of the complicated optical path up to the quad photodiode. Yet looking at the data after the ground loop fix at around slug 78 in Figure 5.16, this effect seemed not to exist at all. The only other item to note here was the need to balance the beam spot size at the Pockels cell (measured $\sim 700 \mu \mathrm{m}$ ) with spot size constraints at the cathode, $\sim 1.5 \mathrm{~mm}$. The tradeoffs 

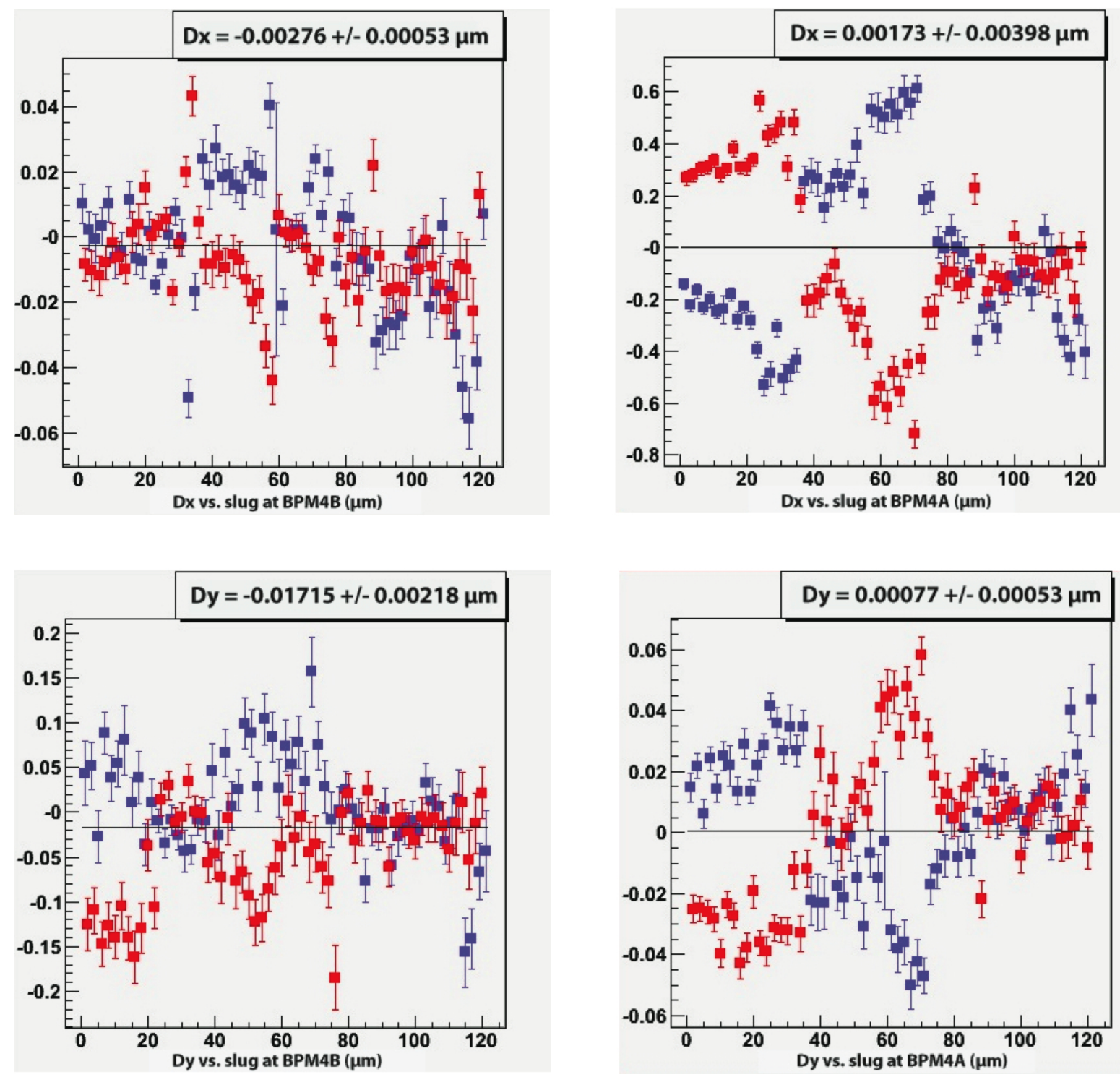

Figure 5.16: $\mathrm{X}$ and $\mathrm{Y}$ position difference data at BPM's 4A and 4B for all 121 slugs of the 2005 ${ }^{4}$ He run. The blue points represent the IHWP "OUT" state while the red do the IHWP "IN" state. 
between a large spot size at the cathode with that at the Pockels cell may be, as previously mentioned, worthy of some further investigation.

\subsubsection{H Tunnel Setup}

The setup for the hydrogen run was very successful, and our understanding of the polarized source was reflected in the RHWP scans. In comparing Figures 5.17 and 5.18, which are plots of $\Delta x(\mu \mathrm{m})$ vs. RHWP angle (degrees) for the first six injector bpm's (known as bpm 's 1I02, 1I04, 1I06, 0I02, 0I02A, and 0I05), the constant term does not flip sign between the IHWP OUT (Figure 5.17) and IN states (Figure 5.18) suggesting dominance by steering; it does flip for bpm 1I02 (the first bpm after the cathode) but the constant is small. The magnitude of the constant offset compared across the bpm's, however, is not the same between the IHWP states suggesting some vacuum window contributions, or possibly statistical error. All $4 \theta$ terms flip sign with the IHWP indicating birefringence gradients in the Pockels cell or analyzing power gradients at the cathode. The $2 \theta$ terms are in almost all cases very small suggesting a RHWP well-matched to the wavelength of the polarized source laser. The one interesting feature about these plots is that there does seem to be a shift in the relative

size of the various beam $A_{\mathrm{LR}}$ 's as you travel to bpm's further downstream. For instance, at bpm1I02, the $4 \theta$ term is large compared to the constant offset, but more equal in size or even smaller than the constant offset at bpm's 0I02 and 0I05. This suggests a shift in what effects seem to dominate the source, but I don't believe anyone has yet provided a coherent explanantion for what is happening to the beam in this region of the injector. The relative size of all of the terms are plausible and how the signs of the terms flip with the insertion of the IHWP are consistent with our current formalism. 
On the RHWP scans where $A_{Q}$ is plotted (Figures 5.19 and 5.20), there is a moderately sized constant that flips sign between IHWP states suggesting a vacuum window contribution. We had to run with a correspondingly moderate sized PITA slope to have the range on the Pockels cell to appropriately minimize this effect. Again, the $4 \theta$ term is larger than the $2 \theta$, suggesting as previously noted dominance by birefringence effects or cathode gradients over some mismatch between the RHWP and wavelength of the source laser.

Figures 5.21-5.24 are plots of $\mathrm{x}$ and $\mathrm{y}$ position differences vs. injector bpm for both IHWP "IN" and "OUT" states up to Faraday Cup 2 of the accelerator right after setup of the source. The position differences plotted here were almost certainly the smallest yet seen in the injector. The y position differences measured just $200 \mathrm{~nm}$ off of the cathode (Figures 5.22 and 5.24) and the evolution of these differences across the injector bpm's was remarkably similar between the two IHWP states. The position differences in $\mathrm{x}$ were even smaller (roughly zero for "OUT" (Fig. 5.21) and $100 \mathrm{~nm}$ for "IN" (Fig. 5.23)). As mentioned in section 5.3.6, we were able to get adiabatic damping factors up to 30 and no less than 5 from the accelerator group, and the position differences averaged over the hydrogen run were the smallest yet achieved. They are displayed in Figure 5.25 which include a sign flip for the IHWP "IN" case in red for the purposes of showing visually how well cancellation was working. The run averaged differences are displayed at the top of each of the four plots.

The IA cell for Hall A was not functioning properly as positive and negative voltage setpoints created a charge asymmetry with the same sign. Given the outstanding results from the source setup, we decided to use PITA voltage setpoints for charge feedback and to abandon the IA system for the experimental run. 


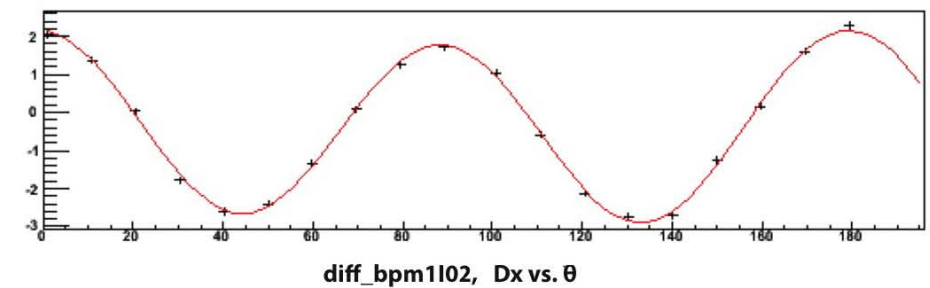

$D x=-0.41+0.23 \sin (2 x+60.76)+2.36 \sin (4 x+95.46)$

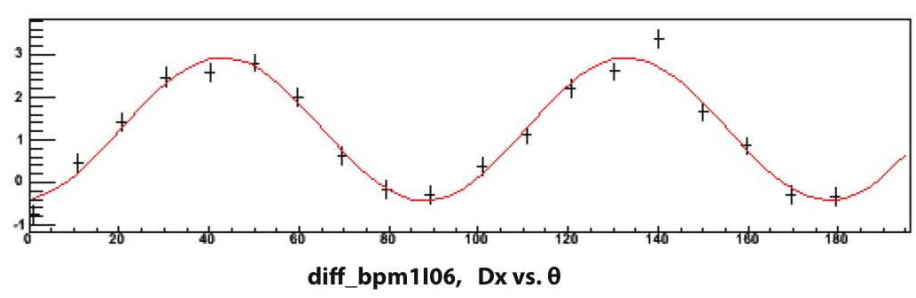

$D x=1.25+0.01 \sin (2 x+86.28)-1.68 \sin (4 x+98.27)$

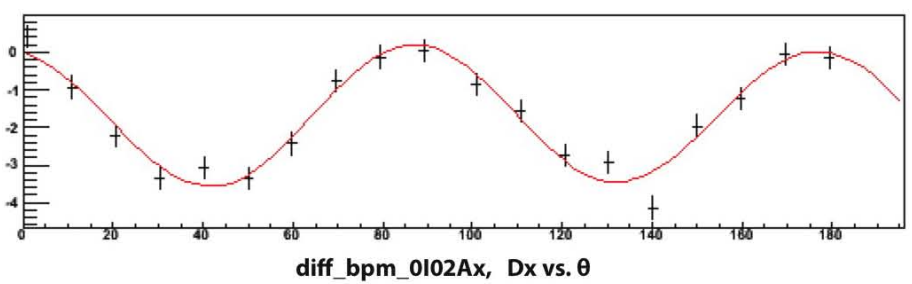

$D x=1.25+0.01 \sin (2 x+86.28)-1.68 \sin (4 x+98.27)$

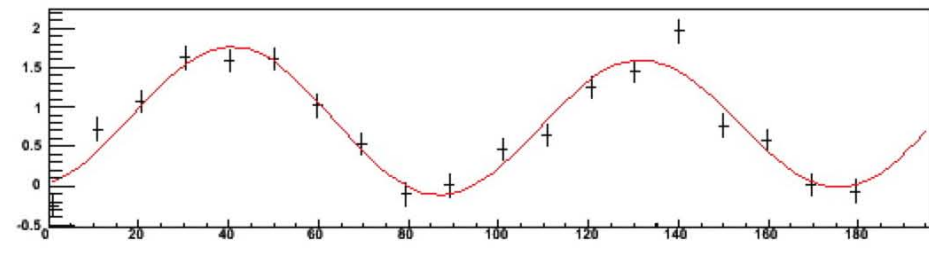

diff_bpm1104x, Dx vs. $\theta$

$D x=0.81+0.10 \sin (2 x+39.67)-0.87 \sin (4 x+105.31)$

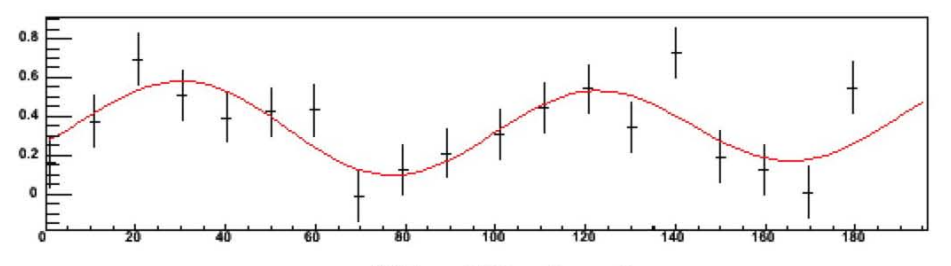

diff_bpm0102x, Dx vs. $\theta$

$D x=0.34+0.04 \sin (2 x+84.00)-0.21 \sin (4 x+144.48)$

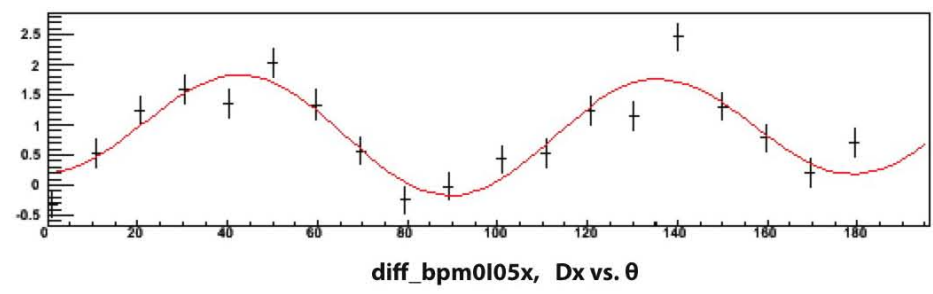

$D x=0.90+0.18 \sin (2 x+80.71)+0.89 \sin (4 x+93.22)$ 


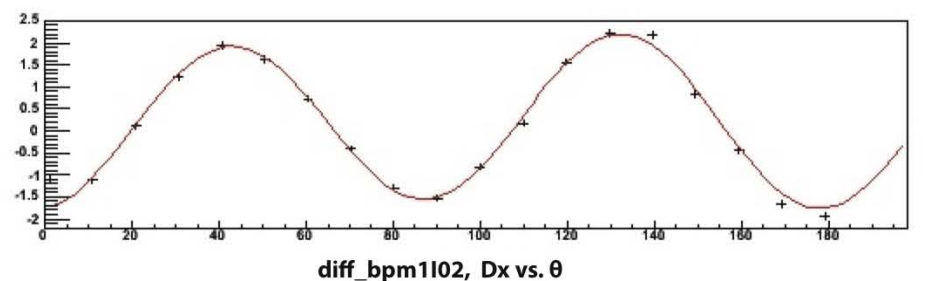

$D x=0.20-0.17 \sin (2 x-43.27)-1.86 \sin (4 x+99.89)$

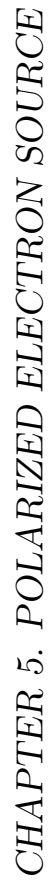

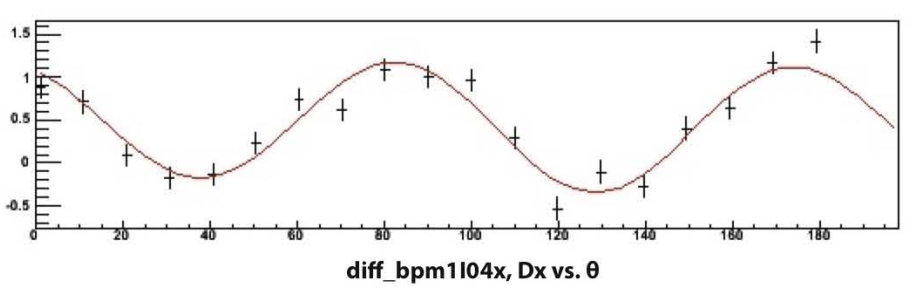

$D x=0.45-0.09 \sin (2 x+173.41)+0.70 \sin (4 x+117.40)$

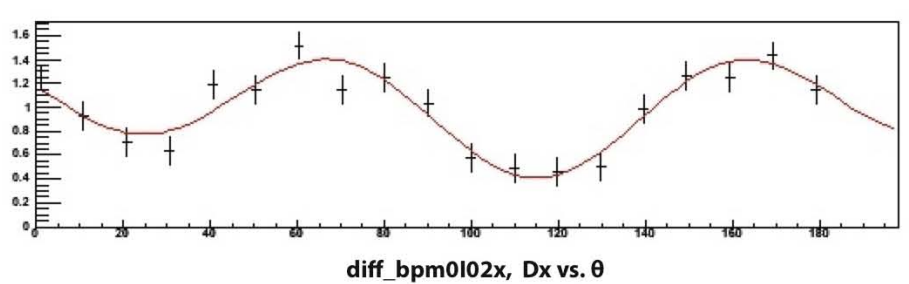

$D x=0.99+0.18 \sin (2 x+38.71)+0.40 \sin (4 x+170.50)$

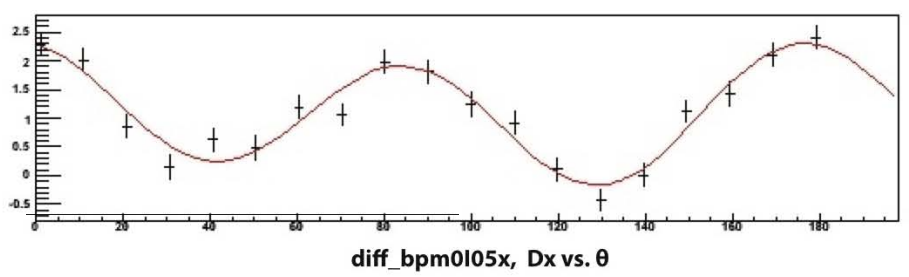

$D x=1.07+0.28 \sin (2 x+53.61)+1.03 \sin (4 x+109.21)$ 


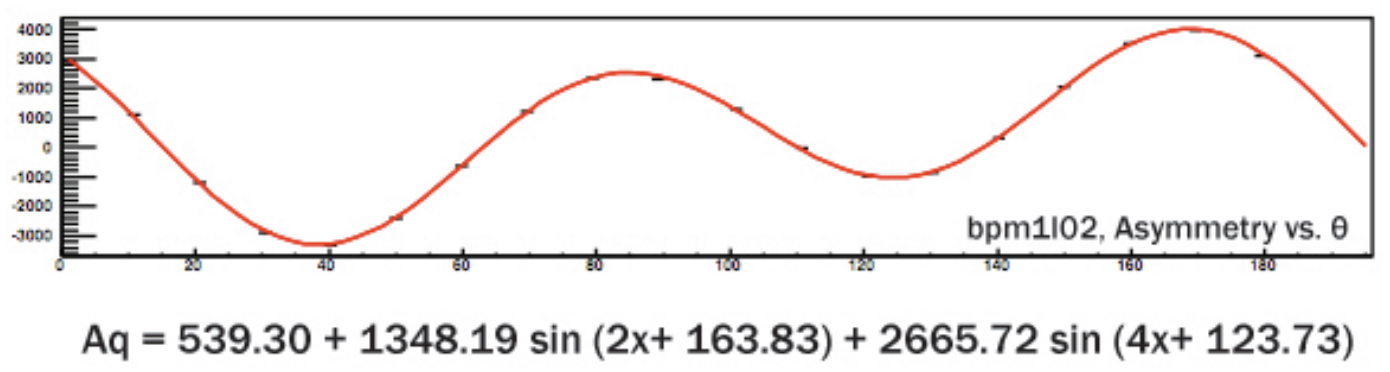

Figure 5.19: $A_{Q}(\mathrm{ppm})$ vs. RHWP angle (degrees) with the IHWP IN taken in the middle of the $2005 \mathrm{H}$ run

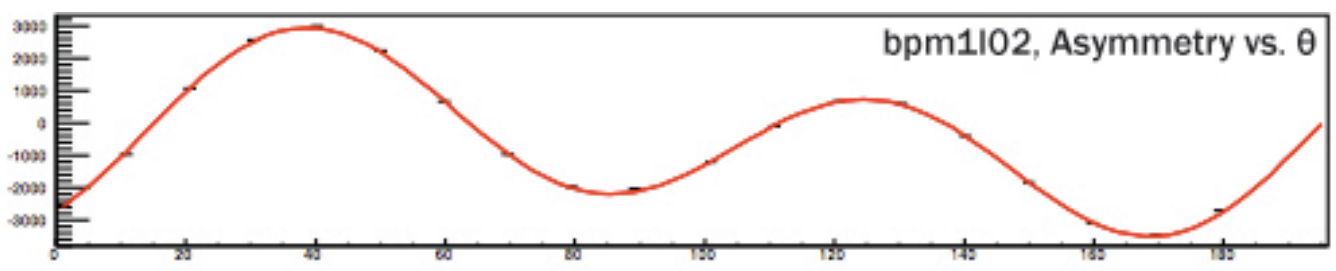

$A q=-482.12+-1288.17 \sin (2 x+165.55)+-2297.43 \sin (4 x+122.69)$

Figure 5.20: $A_{Q}(\mathrm{ppm})$ vs. RHWP angle (degrees) with the IHWP OUT taken in the middle of the $2005 \mathrm{H}$ run

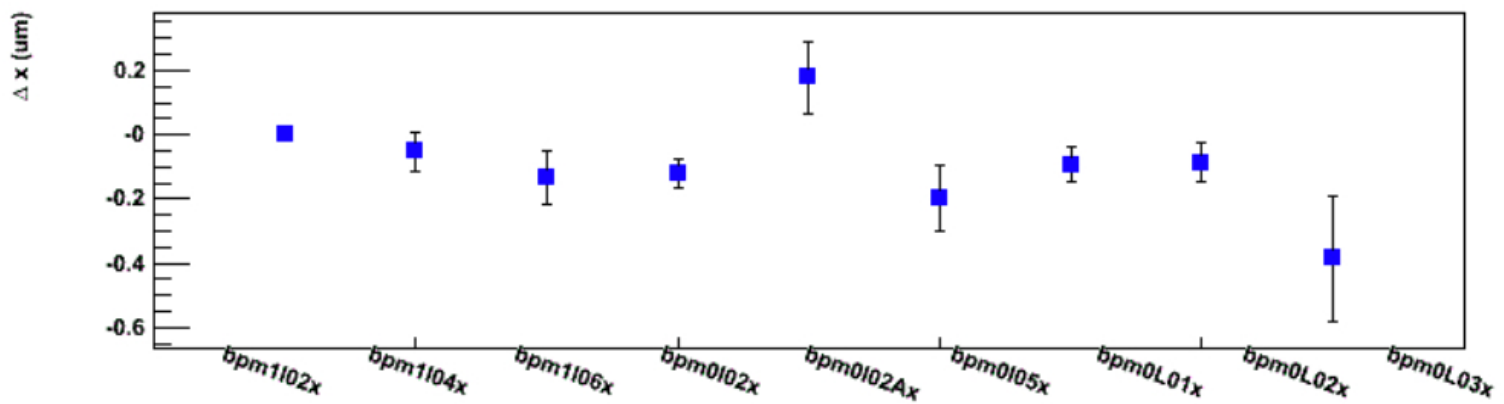

Figure 5.21: X position differences $(\mu \mathrm{m})$ at each of the injector bpm's up to Faraday Cup 2 for IHWP "OUT" 


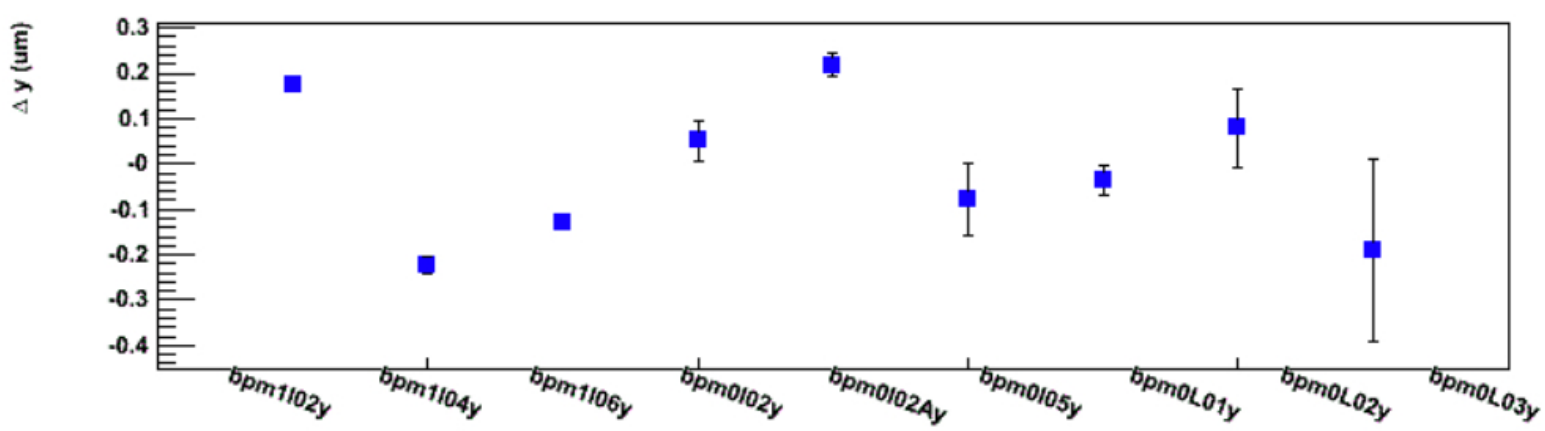

Figure 5.22: Y position differences $(\mu \mathrm{m})$ at each of the injector bpm's up to Farday Cup 2 for IHWP "OUT"

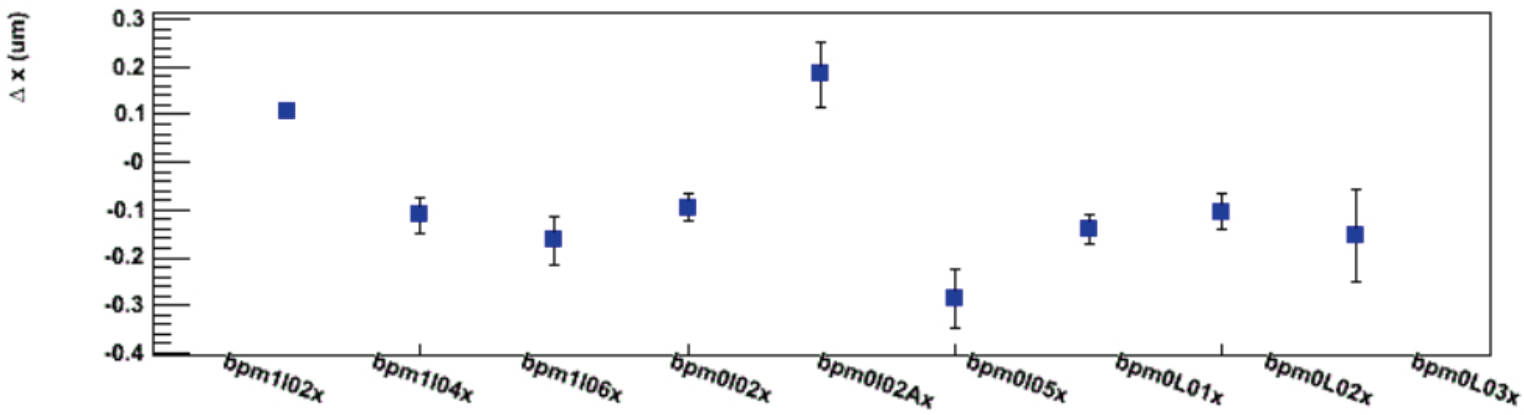

Figure 5.23: X position differences $(\mu \mathrm{m})$ at each of the injector bpm's up to Farday cup 2 for IHWP "IN"

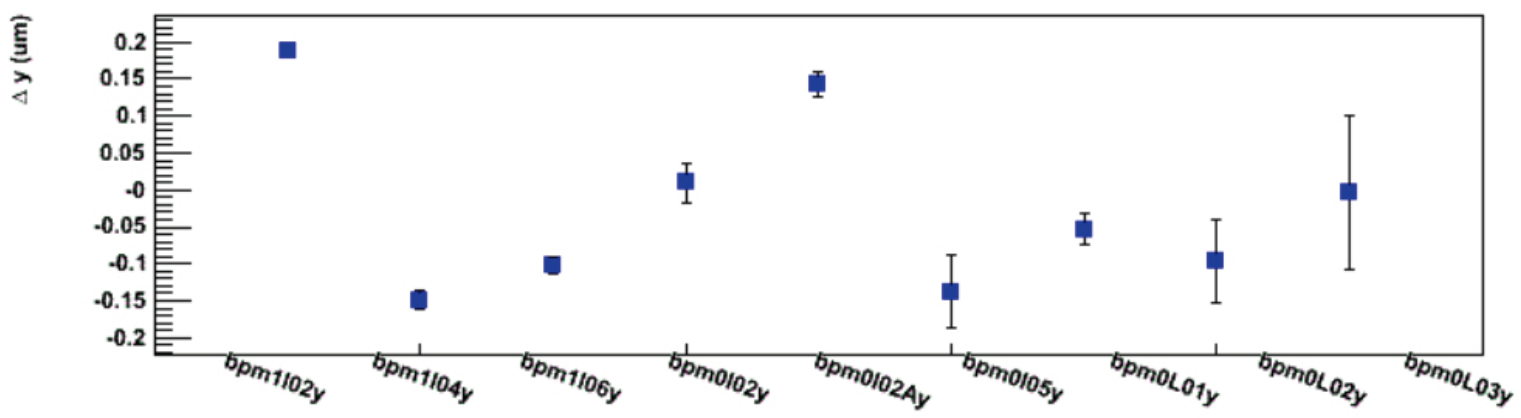

Figure 5.24: Y position differences $(\mu \mathrm{m})$ at each of the injector bpm's up to Farday cup 2 for IHWP "IN" 

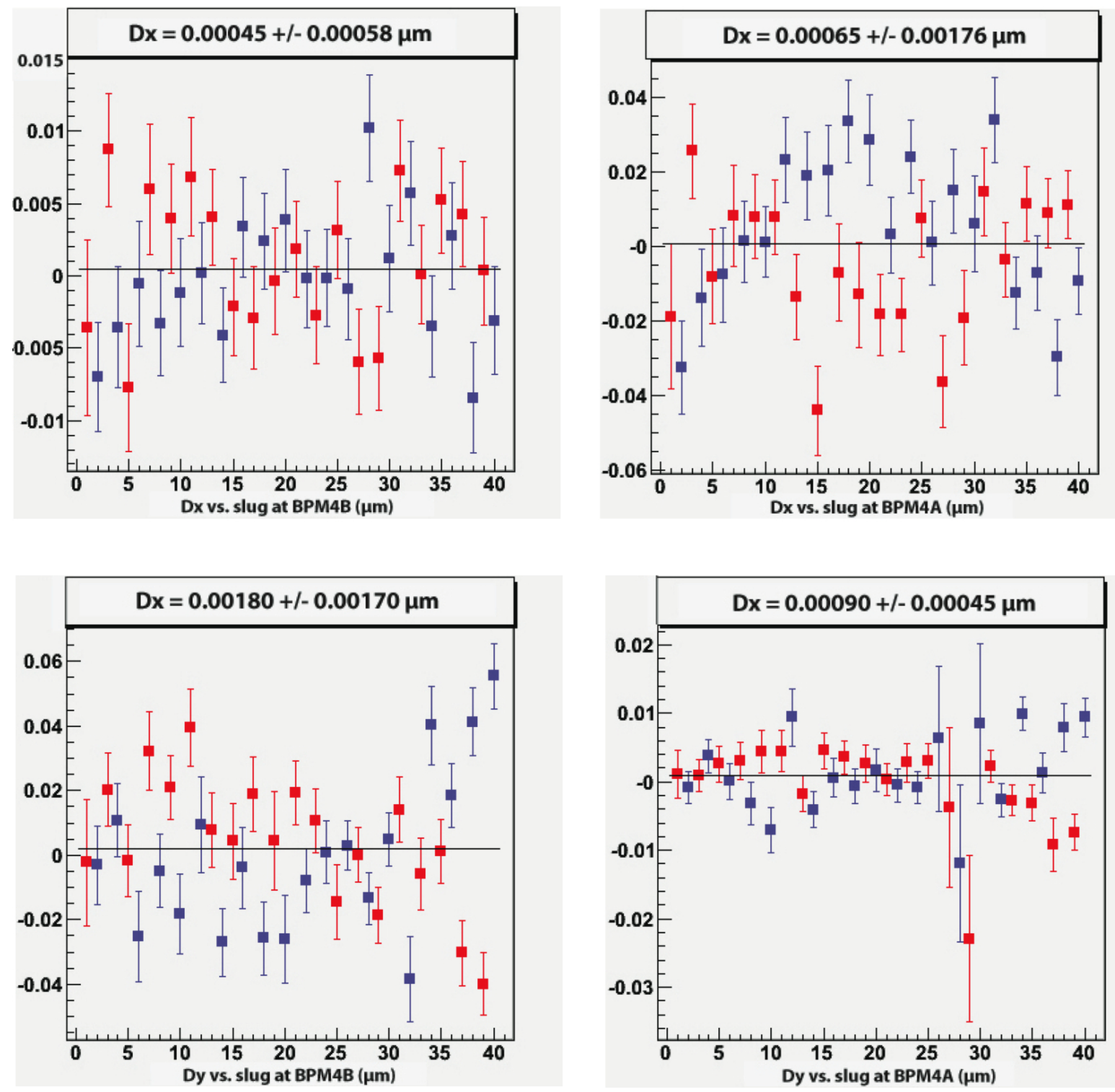

Figure 5.25: $\mathrm{X}$ and $\mathrm{Y}$ position difference data at BPM's 4A and 4B for all 40 slugs of the $2005 \mathrm{H}$ run. The blue points represent the IHWP "OUT" state while the red do the "IN" state. 


\section{Chapter 6}

\section{Analysis}

This chapter will describe the analysis of raw data from the 2005 runs of HAPPEX-He and $-\mathrm{H}$ needed to extract the important physics asymmetries. A detailed description of the background analysis is included in this chapter, along with briefer descriptions of the asymmetry analysis, $Q^{2}$ determination, beam polarization, and effective kinematics. The final physics asymmetry for both the $\mathrm{He}$ and $\mathrm{H}$ runs will be reported in section 6.6.

\subsection{Asymmetry Analysis}

\subsubsection{Raw Asymmetry}

The first step in analyzing the raw asymmetries is making cuts to the data. These cuts involved three separate categories: an inconsistent helicity sequence, a drop in the beam current moved below a certain threshold, and instability in the beam intensity, energy, position, or angle. Analysis of the raw detector asymmetries was carried out using code known as the Parity Analyzer (PAN) [79]. It was written in $\mathrm{C}++$ and utilized ROOT 
[80] libraries and performed many of the necessary analysis tasks: mapped data to the correct monitors, created helicity asymmetries and differences from helicity window pairs, and defined cuts over certain intervals or based on raw beam parameters.

The first type of cut used PAN to check the helicity sequence and verify that the two helicity windows in each pair were indeed of opposite helicity. If this was not satisfied, the window was tagged as "bad" and so were the 25 windows before and after it. The second cut defined a threshold for the beam current below which a cut to the data was applied; this cut also included a certain number of windows before the cut condition was reached as well as after the beam recovered. The third type of cut investigated large excursions by beam parameters that may have caused non-linear responses in the detector. Cuts on these beam instabilities were determined by studying how long it took for detectors and other monitors to recover.

The raw asymmetry detected in each spectrometer arm used the normalized detector flux defined by:

$$
A_{\text {raw }}=\frac{D_{R} / I_{R}-D_{L} / I_{L}}{D_{R} / I_{R}+D_{L} / I_{L}}
$$

where $R, L$ indicates the right, left helicity window, respectively, and $D / I$ is the digitized detector signal $D$ normalized by the digitized signal $I$ from the beam current monitor. Due to target density fluctuations that may have caused correlations between the detectors, the asymmetry for the detector combination was calculated as an asymmetry of the detector average rather than as an average of individual detector asymmetries [49]. The statistical weights for each detector signal take into account differences in the PMT gains from one 

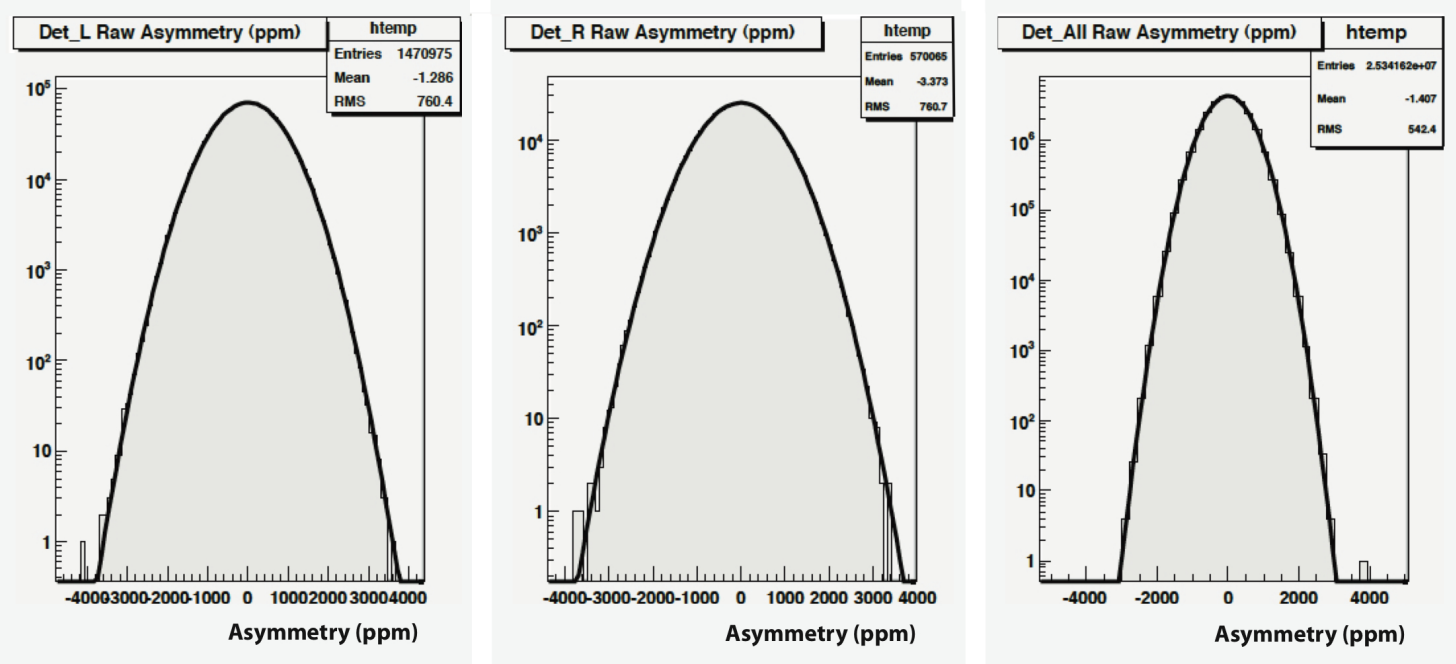

Figure 6.1: $A_{\text {raw }}$ pulse-pair asymmetry distributions for 2005 Hydrogen run plotted on a log scale. Left, right, and all detector combinations are displayed.

detector segment to another as well as differences in the rate of flux into the detector. These detector combinations will be used to calculate the final asymmetry described later in this chapter.

\subsubsection{Beam Modulation Analysis}

The beam modulation technique discussed in section 3.8 was used to correct the components of the raw asymmetry arising from helicity-correlated beam asymmetries in position, angle, and energy at the target. The beam position monitors 4BX, 4BY, 4AX, 4AY, and $12 \mathrm{X}$ allow nearly orthogonal observation of these parameters. Corrections to the asymmetry based upon these helicity correlated beam asymmetries were calculated from

$$
\Delta A=\sum_{i=1}^{5}\left(\frac{\partial \sigma}{\partial M_{i}}\right) \Delta M_{i}
$$


where $\sigma$ is the cross section measured by a detector, the slope $\frac{\partial \sigma}{\partial M_{i}}$ is the normalized detector sensitivity to the $i^{\text {th }}$ beam position monitor, and $\Delta M_{i}$ is the measured helicity correlated beam position difference at one of those monitors. The $\Delta M_{i}$ are minimized according to the discussion in Chapter 5. The slopes were determined by expanding the detector sensitivity to include its response to the $j^{\text {th }}$ modulation coil in terms of the beam position monitors,

$$
\frac{\partial \sigma}{\partial C_{j}}=\sum_{i=1}^{5}\left(\frac{\partial \sigma}{\partial M_{i}}\right)\left(\frac{\partial M_{i}}{\partial C_{j}}\right)
$$

where $(\delta \sigma) /\left(\delta M_{i}\right)$, the response of the detector to changes in the beam parameters, is the quantity in which we are interested. $\partial \sigma / \partial M_{i}$ was extracted by first defining the $\chi^{2}$ :

$$
\chi^{2}=\sum_{j}\left[\left(\frac{\partial \sigma}{\partial C_{j}}-\sum_{i=1}^{5} \frac{\partial \sigma}{\partial M_{i}} \frac{\partial M_{i}}{\partial C_{j}}\right) / \sigma^{2}\right]^{2}
$$

and minimizing it with respect to $\partial \sigma / \partial M_{i}$ :

$$
\sum_{j}\left(\frac{\partial \sigma}{\partial C_{j}} \frac{\partial M_{k}}{\partial C_{j}}\right) / \sigma^{2}=\frac{\partial \sigma}{\partial M_{i}} \cdot \sum_{j}\left(\frac{\partial M_{i}}{\partial C_{j}} \frac{\partial M_{k}}{\partial C_{j}}\right) / \sigma^{2}
$$

This relationship can be rewritten as

$$
\mathbf{D}_{\mathbf{C}}=\mathbf{D}_{\mathbf{M}} \cdot \mathbf{M}_{\mathbf{C}}
$$




\begin{tabular}{|l|r|r|r|r|r|}
\hline & 4bx & 4by & 4ax & 4ay & 12x \\
\hline \hline det1 & 61.8 & 32.5 & 38.7 & -13.6 & -45.4 \\
det2 & -39.5 & -5.0 & 0.7 & 2.6 & 11.7 \\
det3 & 92.7 & 9.1 & -16.8 & -3.4 & -14.6 \\
det4 & -67.8 & -8.8 & 1.6 & 4.0 & 17.3 \\
det_l & -18.6 & 2.7 & 8.5 & -0.7 & -0.1 \\
det_r & 14.5 & 0.4 & -7.8 & 0.2 & 1.0 \\
det_all & 3.5 & 1.2 & -2.4 & -0.2 & 0.6 \\
\hline
\end{tabular}

Table 6.1: 2005 HAPPEX-H average beam modulation slopes for each beam position monitor in units of $\mathrm{ppm} / \mu \mathrm{m}$.

where

$$
\begin{aligned}
\mathbf{D}_{\mathbf{C}} & =\sum_{j}\left(\frac{\partial \sigma}{\partial C_{j}} \frac{\partial M_{k}}{\partial C_{j}}\right) / \sigma^{2} \\
\mathbf{D}_{\mathbf{M}} & =\frac{\partial \sigma}{\partial M_{i}} \\
\mathbf{M}_{\mathbf{C}} & =\sum_{j}\left(\frac{\partial M_{i}}{\partial C_{j}} \frac{\partial M_{k}}{\partial C_{j}}\right) / \sigma^{2} .
\end{aligned}
$$

So, the calculation of detector sensitivities to position differences involves just a matrix inversion:

$$
\mathbf{D}_{\mathbf{M}}=\mathbf{D}_{\mathbf{C}} \cdot \mathbf{M}_{\mathbf{C}}^{-1}
$$

A typical beam modulation cycle is shown in Figure 6.2, and the detector sensitivities measured for HAPPEX-H are displayed in Table 6.1. The individual detector segments are 5-20 times more sensitive than the combination of them as the elastic peak is divided among the detector segments.

The 2005 HAPPEX-He and -H position differences and corrections based on detector 

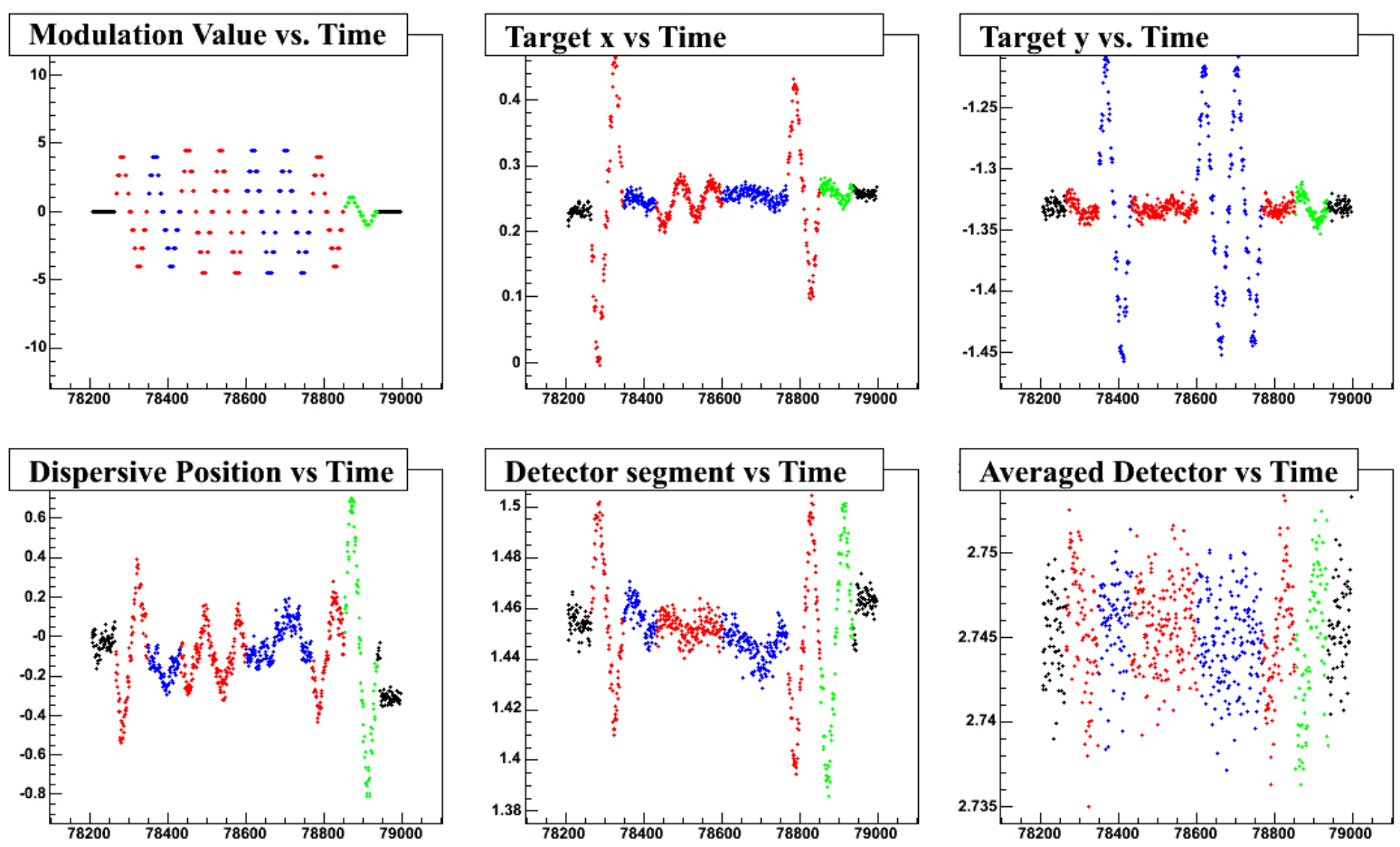

Figure 6.2: A beam modulation cycle: the red data are from modulation in $x$ and the blue from modulation in $y$. The plot in the top left shows modulation value vs. event number and the remaining plots display BPM and detector response vs. event number.

sensitivities to them are displayed in Table 6.2. When both spectrometer arms are operating during production running, the all detector combination corrections are applied to the data.

If only one arm is running, however, than the correction for either left or right is used. Due to the good statistical behavior of detector asymmetries and their differences, we concluded the energy differences at $12 \mathrm{x}$ were well understood and no systematic error was assigned. The largest corrections here were from the BPM4ax and BPM4bx numbers, and we attached a $100 \%$ systematic error of $10 \mathrm{ppb}$ to each. Added in quadrature, the total systematic error on the correction to the hydrogen raw asymmetry is $14 \mathrm{ppb}$. The corrected data for each hydrogen dataset (slug) and the run averaged correction arranged by $\lambda / 2$ plate state is displayed in Figure 6.3. 


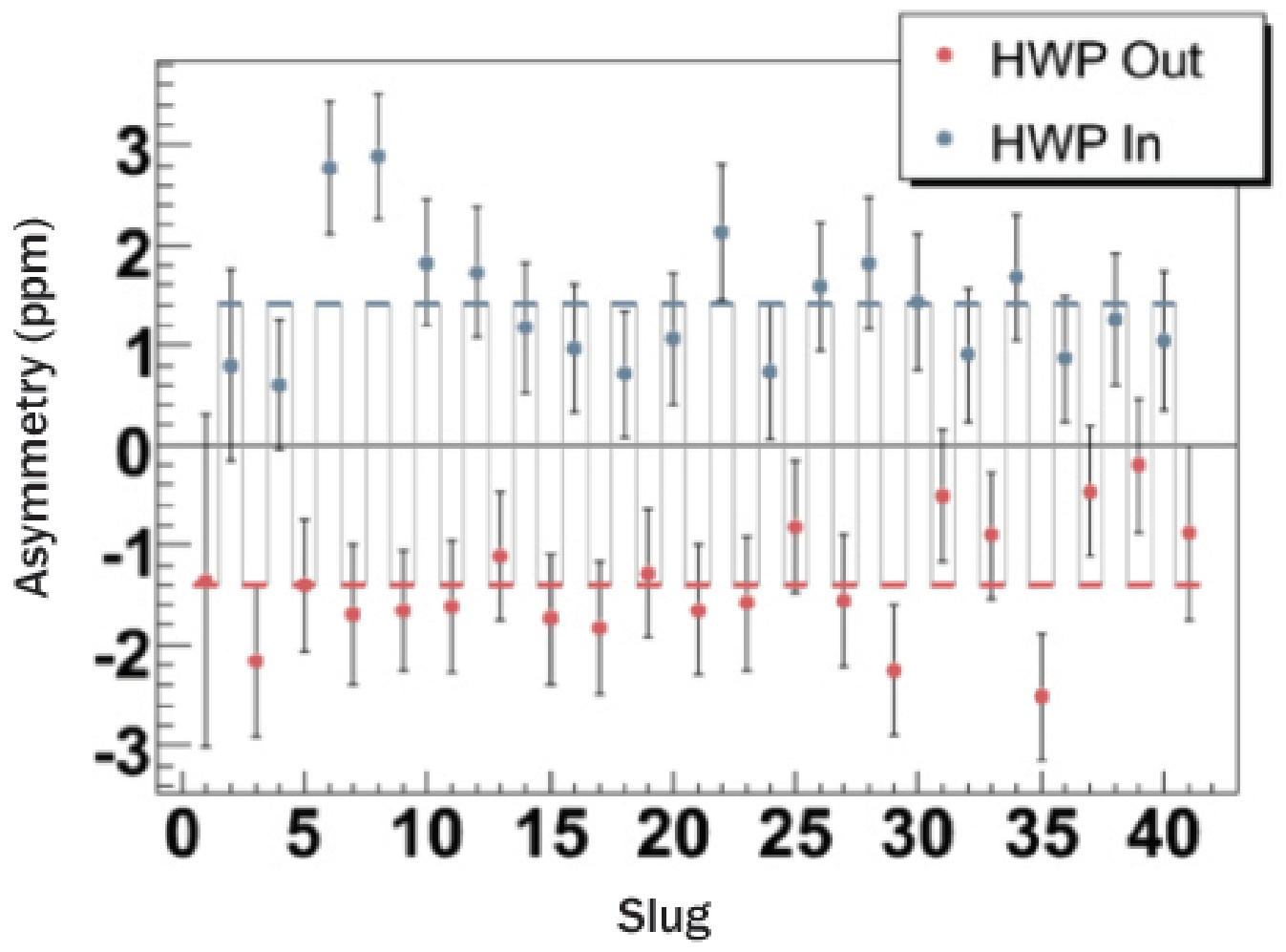

Figure 6.3: $A_{\text {corr }}$ for HAPPEX-He 2005 data arranged by IHWP state for sequential data sets (slugs). The dashed line in both red and blue represents $A_{\text {corr }}$, averaged over the entire run and plotted with the correct sign for each $\lambda / 2$ plate state. 


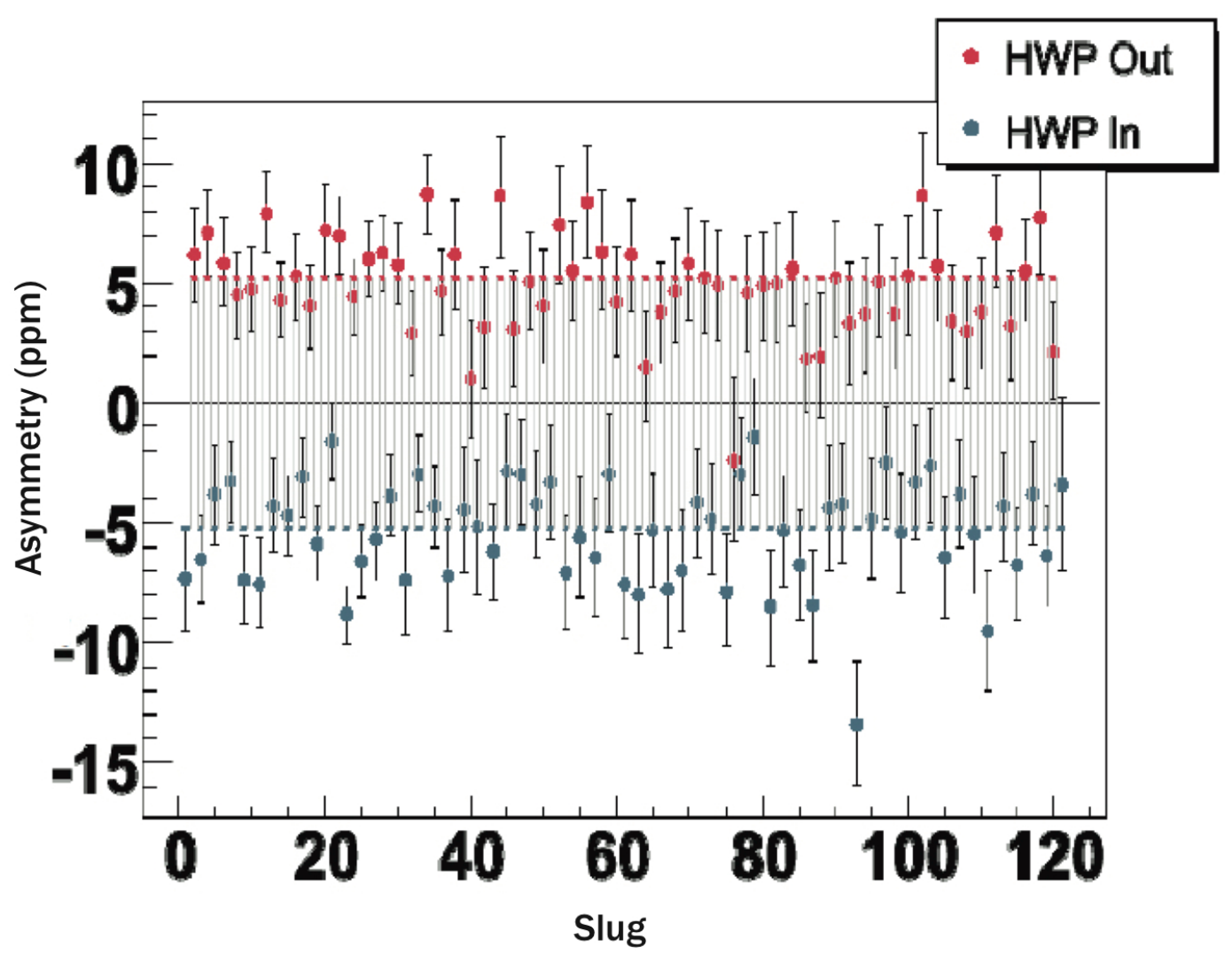

Figure 6.4: $A_{\text {corr }}$ for HAPPEX-H 2005 data arranged by $\lambda / 2$ plate state for sequential data sets (slugs). The dashed line in both red and blue represents $A_{\text {corr }}$, averaged over the entire run and plotted with the correct sign for each $\lambda / 2$ plate state. 


\begin{tabular}{|c|rl|rl|}
\hline & \multicolumn{2}{|c|}{ HAPPEX-He } & \multicolumn{2}{|c|}{ HAPPEX-H } \\
\hline Energy & 3 & $\mathrm{ppb}$ & 0.2 & $\mathrm{ppb}$ \\
\hline$\Delta x$ & -0.2 & $\mathrm{~nm}$ & 0.5 & $\mathrm{~nm}$ \\
\hline$\Delta x^{\prime}$ & 4.4 & $\mathrm{nrad}$ & -0.2 & $\mathrm{nrad}$ \\
\hline$\Delta y$ & -26 & $\mathrm{~nm}$ & 1.7 & $\mathrm{~nm}$ \\
\hline$\Delta y^{\prime}$ & -4.4 & $\mathrm{nrad}$ & 0.2 & $\mathrm{nrad}$ \\
\hline Left Corrections & -370 & $\mathrm{ppb}$ & -10 & $\mathrm{ppb}$ \\
\hline Right Corrections & 80 & $\mathrm{ppb}$ & -10 & $\mathrm{ppb}$ \\
\hline All Corrections & 120 & $\mathrm{ppb}$ & -10 & $\mathrm{ppb}$ \\
\hline
\end{tabular}

Table 6.2: Average beam asymmetries for energy, and position and angle differences in the $\mathrm{x}$ and $\mathrm{y}$ dimensions for the 2005 HAPPEX-II runs. The bottom three rows display the corrections applied to the raw asymmetry for right and left arm only data as well as both arms running, labeled "All Corrections".

\begin{tabular}{|c|c|c|c|}
\hline & IHWP OUT & IHWP IN & BOTH \\
\hline \hline \multicolumn{3}{|c|}{ Helium } \\
\hline$A_{\text {raw }}$ & $4.80 \pm 0.27$ & $-5.41 \pm 0.27$ & $5.10 \pm 0.19$ \\
$A_{\text {corr }}$ & $5.12 \pm 0.27$ & $-5.38 \pm 0.27$ & $5.25 \pm 0.19$ \\
\hline \hline \multicolumn{4}{|c|}{ Hydrogen } \\
\hline$A_{\text {raw }}$ & $-1.40 \pm 0.15$ & $1.42 \pm 0.15$ & $-1.41 \pm 0.11$ \\
$A_{\text {corr }}$ & $-1.41 \pm 0.15$ & $1.43 \pm 0.15$ & $-1.42 \pm 0.11$ \\
\hline
\end{tabular}

Table 6.3: Raw and corrected asymmetries (in ppm) displayed by IHWP state. The differences between $A_{\text {raw }}$ and $A_{\text {corr }}$ result from corrections for energy, position, and angle differences.

For ${ }^{4} \mathrm{He}$, the errors on the beam asymmetry corrections were as follows: errors on them were as follows: $20 \mathrm{ppb}$ for BPM4Bx, $10 \mathrm{ppb}$ for BPM4By, $30 \mathrm{ppb}$ for BPM4Ax, and 10 ppb for $12 \mathrm{x}$. The error on BPM4Bx was proposed due to the lack of cancellation at that monitor; that on BPM4Ax due to the large corrections that were made over the course of the run; and that on 12x due to a small discrepancy between the left and right detector slopes. Added in quadrature, the total systematic error on correction to the helium asymmetry was $37 \mathrm{ppb}$. The corrected data for each ${ }^{4} \mathrm{He}$ dataset (slug) and the run averaged correction arranged by $\lambda / 2$ plate state is displayed in Figure 6.4 .

Figure 6.5 shows all the final data from the 2005 runs of HAPPEx-H and -He. The data for hydrogen is displayed uncorrected, but with only a -10 ppb correction, the difference from 

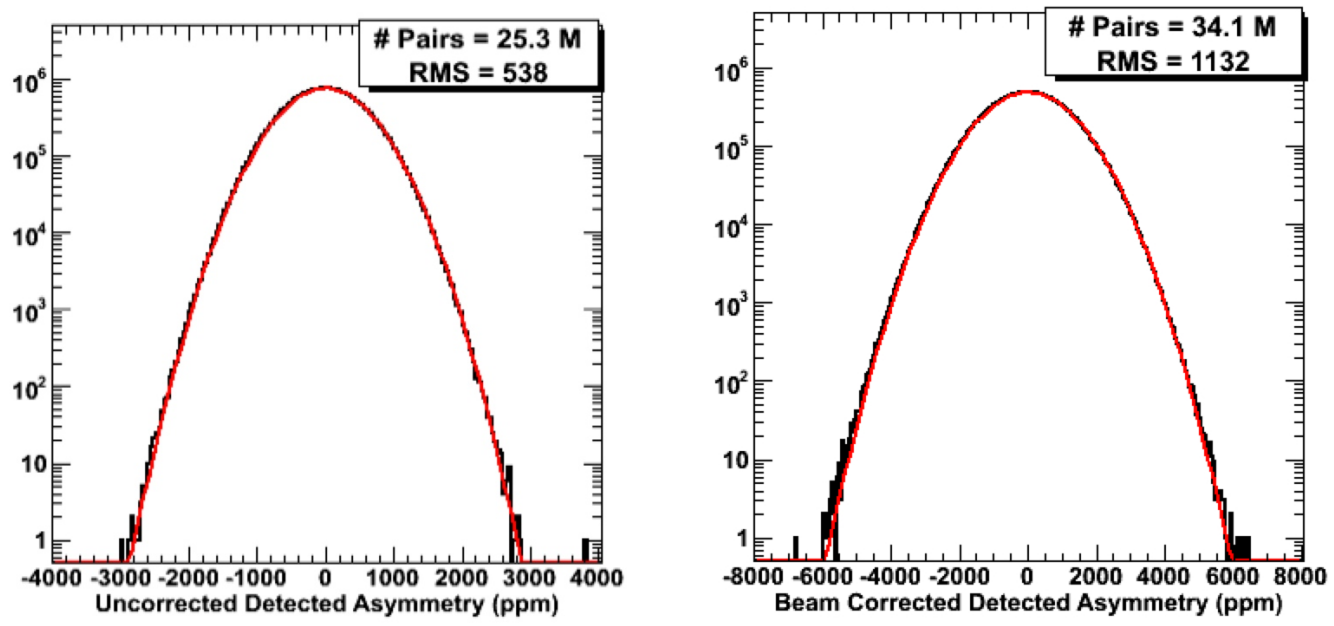

Figure 6.5: The ${ }^{1} \mathrm{H}$ uncorrected (left plot) and ${ }^{4} \mathrm{He}$ corrected (right plot) asymmetries for all of the final data for the 2005 HAPPEX runs. The number of window pairs and detector asymmetry are displayed in each plot.

the corrected is indistinguishable. The final data consisted of $35.0 \times 10^{6}$ ( ${ }^{4} \mathrm{He}$ ) and $26.4 \mathrm{x}$ $10^{6}$ (Hydrogen) pairs with the final detector RMS's being 538 and 1132 ppm, respectively.

The final corrected asymmetry is calculated from

$$
\left\langle A_{\text {corr }}\right\rangle=\frac{\sum_{n}\left\langle A_{\text {corr }}\right\rangle_{n} /\left(\sigma_{n}^{\text {corr }}\right)^{2}}{\sum_{n} 1 /\left(\sigma_{n}^{\text {corr }}\right)^{2}}
$$

where $\left\langle A_{\text {corr }}\right\rangle_{n}$ and $\sigma_{n}^{\text {corr }}$ are the average corrected asymmetry and error, respectively, for run $n$. The raw and corrected asymmetries broken up by $\lambda / 2$ plate state for both ${ }^{4} \mathrm{He}$ and Hydrogen are displayed in Table 6.3.

The remaining sections (6.1.3 and 6.1.4) discuss other false asymmetries, but their effect was so negligible that no correction was made to account for them. 


\subsubsection{Linearity}

Ideally, a flux-integrating detector will output a signal proportional to the incoming scattered flux; in reality, however, any nonlinearity in the PMT response to the incoming flux can be a significant systematic error. The nonlinearity in a detector's response $D$ to the flux $F$ can be approximated as:

$$
D(F)=F+\epsilon F^{2}
$$

It can be shown that that the raw asymmetry is

$$
A_{\text {raw }}=A_{P V}+X\left(A_{P V}+A_{Q}\right)
$$

where $X=\epsilon F$ with $|\epsilon| \ll 1$ and $A_{Q}$ and $A_{P V}$ are the charge and physics asymmetries respectively defined as

$$
A_{Q}=\frac{I_{R}-I_{L}}{I_{R}+I_{L}} \text { and } A_{P V}=\frac{\sigma_{R}-\sigma_{L}}{\sigma_{R}+\sigma_{L}}
$$

From Equation 6.11, it is clear that the nonlinearity correction can be made small if $A_{Q}$ can be kept much smaller than $A_{P V}$. The resulting fractional error in the asymmetry is equal to $X$.

If we considered only the unnormalized detector asymmetry, corrections to it would appear as

$$
A_{\mathrm{corr}}=A_{\mathrm{det}}-A_{Q}+\sum_{i=1}^{5} \beta_{i} \Delta x_{i}
$$


and corrections to the linearity would need to take into account that of both the detector as well as the BCM, turning Eqn. 13 into

$$
A_{\mathrm{corr}}=\left(\frac{1}{L_{Q}}\right)\left(\frac{1}{L_{D}}\right) A_{\mathrm{det}}-\left(\frac{1}{L_{Q}}\right) A_{Q}+\sum_{i=1}^{5} \beta_{i} \Delta x_{i}
$$

where $L_{Q}$ is $\left(1-X_{Q}\right)$ is the BCM linearity and $L_{D}$ is $\left(1-X_{D}\right)$, the individual detector linearity.

The BCM nonlinearity is measured by taking a "double difference" from two BCM's, one upstream and another downstream. The detector nonlinearity is measured by fitting a plot of the normalized detector asymmetry versus charge asymmetry to a straight line; the measured slope of the fit was the nonlinearity. The BCM's had their pedestals fit versus the Unser monitor using current ramps, and the nonlinearity of the BCM at low currents was taken into account.

For both ${ }^{1} \mathrm{H}$ and ${ }^{4} \mathrm{He}$, the detector and BCM's were linear with upper limits of uncertainty of $1 \%$ and $2 \%$, respectively. The error on the asymmetry due to the detector is its uncertainty in the linearity multiplied by the raw asymmetry (5.25 ppm for ${ }^{4} \mathrm{He}$ and $-1.418 \mathrm{ppm}$ for ${ }^{1} \mathrm{H}$ ) and that of the BCM is its linearity uncertainty multiplied by the charge asymmetry, $A_{Q}$ (-0.38 ppm for ${ }^{4} \mathrm{He}$ and $0.41 \mathrm{ppm}$ for $\left.{ }^{1} \mathrm{H}\right)$.

\subsubsection{Transverse Beam Asymmetry}

The interference between one- and two-photon exchange when elastically scattering transversely polarized electrons from an unpolarized target gives rises to a transverse beam asym- 
metry, $A_{T}$. The asymmetry arises from an azimuthal modulation as

$$
A_{T} \propto \vec{S}_{e} \cdot \frac{\vec{k}_{e} \times \vec{k}_{e}^{\prime}}{\left|\vec{k}_{e} \times \vec{k}_{e}^{\prime}\right|}
$$

where $\vec{S}_{e}$ and $\vec{k}_{e}$ are the spin and momentum vector, respectively, of the incoming electron, and $\vec{k}_{e}^{\prime}$ is the momentum vector of the scattered electron. This asymmetry was of concern for us due to the transverse component of the polarized beam; and yet the horizontal acceptance of the HRS meant that we were only sensitive to the vertically polarized component. Since $A_{T}$ has an opposite sign in the left and right detectors, it will contribute a false asymmetry to $A_{P V}$ if it does not cancel when the detectors are averaged.

The $A_{T}$ for hydrogen $\left(A_{T}^{H}\right)$ was measured in 2004 with $3.1 \times 10^{5}$ window pairs and a detector width of $610 \mathrm{ppm}$ for two slugs. After correcting for polarization (75\%) and sign, the average for both detector arms was

$$
A_{T}^{H}=-6.58 \pm 1.47 \text { (stat) } \pm 0.24 \text { (syst) ppm. }
$$

The vertical polarization in 2005 for hydrogen was $0.0 \pm 2 \%$. The helium measurement was made in 2005 with a vertical polarization of $3.6 \%$ during the July/August running, but was estimated at $1.7 \pm 1.8 \%$ for the the entire run with the inclusion of the September period. The averaged $A_{T}$ for helium was

$$
A_{T}^{H e}=-13.5 \pm 1 \mathrm{ppm} .
$$

A description of how the vertical polarization $P_{v}$ was measured can be found in [81]; if 
$P_{v}$ is known, the total contribution of $A_{T}$ to $A_{P V}$ can be determined. A correction to $A_{\text {raw }}$ was unnecessary for both ${ }^{4} \mathrm{He}$ and ${ }^{1} \mathrm{H}$ as its size was so much less than the statistical error. Uncertainties in the knowledge of left/right cancellation, $P_{v}$, and left/right differences in the asymmetry (kinematics), all contributed to the systematic error and resulted in estimates of $8 \mathrm{ppb}$ for ${ }^{4} \mathrm{He}$ and $4 \mathrm{ppb}$ for ${ }^{1} \mathrm{H}$.

\section{2 $Q^{2}$ Determination}

The four-momentum transfer squared is

$$
Q^{2}=2 E E^{\prime}(1-\cos \theta)
$$

where the three ingredients needed are the incident beam energy $E$, the final energy of the electron $E^{\prime}$, and the scattering angle $\theta$. This section details how each of these were measured to determine $Q^{2}$, a crucial value given that the physics asymmetry is linearly dependent on it.

The "Tiefenbach" energy was used for $E$ in this measurement; it is calculated from the current values of the Hall A arc Bdl value and Hall A beam position monitors (BPMs). This number is continuously recorded in the data stream. An error of $3 \mathrm{MeV}$ was assigned to both helium and hydrogen beam energies, a conservative value based on the history of discrepancies of energy measurements (both Arc method and eP measurement, see Section 4.3.1) and drifts in the accelerator setup.

Being at a very forward angle, the largest systematic error contribution is from the scattering angle. A more accurate method for determining this angle than can be achieved 
through surveying was suggested by Nilanga Liyanage, and it relies on measuring the energy of elastically scattered electrons from different nuclei to determine the angle of the spectrometers [83]. The scattered electrons from only the central hole of a $0.5 \mathrm{~m}$ think tungsten plate with a rectangular pattern of holes (sieve slit) covering the acceptance were compared for multiple targets and multiple momentum settings.

This relationship between the energy of a scattered electron $E^{\prime}$, beam energy $E_{o}$, scattering angle $\theta$, and mass of the initial $(m)$ and final $\left(m^{*}\right)$ recoiling nucleus is determined from

$$
E^{\prime}+\epsilon^{\prime}=\frac{E_{o}-\epsilon_{o}-\frac{1}{2 m}\left(m^{* 2}-m^{2}\right)}{1+\left(E_{o}-\epsilon_{o}\right) / m(1-\cos \theta)}
$$

where $\epsilon_{o}$ and $\epsilon^{\prime}$ are the energy losses due to ionization within the target for the incident and detected electrons. It is common to set $\epsilon^{\prime}=\epsilon_{o}$ and assign each half the energy loss due to ionization in the target. These losses were calculated from the Bethe-Block equation.

The energy loss due to bremmstrahlung was accounted for in the shape of the shape of the electron energy spectrum peaks. The peak shape was chosen as a gaussian convoluted with an exponential [83], and needed for this fit is the reconstructed energy of the scattered electron,

$$
x=P_{o}(1+\delta+\Delta \delta)
$$

Here, $P_{o}$ is the central momentum of the spectrometer; $\delta$ is the fractional difference of the reconstructed momentum from $P_{o}$; and $\Delta \delta$ is the second-order correction to $\delta$ that was 


\begin{tabular}{|l|l|} 
& \\
\hline $\begin{array}{l}{ }^{4} \text { He HAPPEX } \\
\text { Beam Energy } \\
E-<x \frac{d E}{d x}>\end{array}$ & $2.7503 \mathrm{GeV}$ \\
L-arm Angle & $6.06^{\circ}$ \\
L-arm Momentum & $2.7361 \mathrm{GeV}$ \\
R-arm Angle & $6.12^{\circ}$ \\
R-arm Momentum & 2.7346 \\
\hline Hydrogen HAPPEX & \\
Beam Energy & $3.176 \mathrm{GeV}$ \\
E- $<x \frac{d E}{d x}>$ & \\
L-arm Angle & $6.043^{\circ}$ \\
L-arm Momentum & $3.1234 \mathrm{GeV}$ \\
R-arm Angle & $6.106^{\circ}$ \\
R-arm Momentum & 3.1126 \\
\hline
\end{tabular}

Table 6.4: Inputs to $Q^{2}$

found to be necessary when considering imperfections in the spectrometer's reconstruction of matrix elements [83]. The reliability of the reconstructed $\delta$ and momentum was checked by comparing the scattered electron spectra off of carbon and water targets for different momentum settings of the spectrometer. The carbon and water-cell spectra were fit for the central hole for each kinematic setting, with the gaussian convoluted with the exponential function describing the shape of each peak. The "true" location of each peak is calculated from Equation 6.19 using the known values of $E, m, m^{*}$, and "trial values" of $\theta$ [83]. The inputs used for all iterations of this calculation are shown in Table 6.4. Table 6.5 gives the fixed parameters used in the fit of the $E^{\prime}$ distributions. A sample fit to the central sieve slit hole is shown in Figure 6.6.

The average angle measured by the spectrometers for ${ }^{4} \mathrm{He}$ was $6.06 \pm 0.01^{\circ}$ and $6.12 \pm$ $0.01^{\circ}$ for the left and right arms, respectively. The weighted average of these two was $6.09^{\circ}$. For ${ }^{1} \mathrm{H}$, the measured angles were $6.04 \pm 0.01^{\circ}$ for the left and right arms, respectively. The 


\begin{tabular}{|lr|}
\hline Parameters & Value $(\mathrm{MeV})$ \\
\hline$E_{0}$ & $3025 . \triangle \theta=0.002^{\circ} / \mathrm{MeV}$ \\
\hline$m\left({ }^{1} \mathrm{H}\right)$ & 938.27 \\
\hline$m\left({ }^{16} \mathrm{O}\right)$ & 14895.08 \\
\hline$m\left({ }^{16} \mathrm{O}_{3 m}\right)-m\left({ }^{16} \mathrm{O}\right)$ & 6.13 \\
\hline$m\left({ }^{16} \mathrm{O}_{2 p 1 m}\right)-m\left({ }^{16} \mathrm{O}\right)$ & 7.12 \\
\hline$m\left({ }^{12} \mathrm{C}\right)$ & 11174.86 \\
\hline$m\left({ }^{12} C_{2 p}\right)-m\left({ }^{12} \mathrm{C}\right)$ & 4.44 \\
\hline$m\left({ }^{12} C_{0 p}\right)-m\left({ }^{12} \mathrm{C}\right)$ & 7.65 \\
\hline$m\left({ }^{12} C_{3 m}\right)-m\left({ }^{12} \mathrm{C}\right)$ & 9.64 \\
\hline$m\left({ }^{56} \mathrm{Fe}\right)$ & 52089.78 \\
\hline$m\left({ }^{56} \mathrm{Fe} e_{2 p}\right)-m\left({ }^{56} \mathrm{Fe}\right)$ & 4.32 \\
\hline
\end{tabular}

Table 6.5: The fixed parameters used in the fit of the $E^{\prime}$ distributions

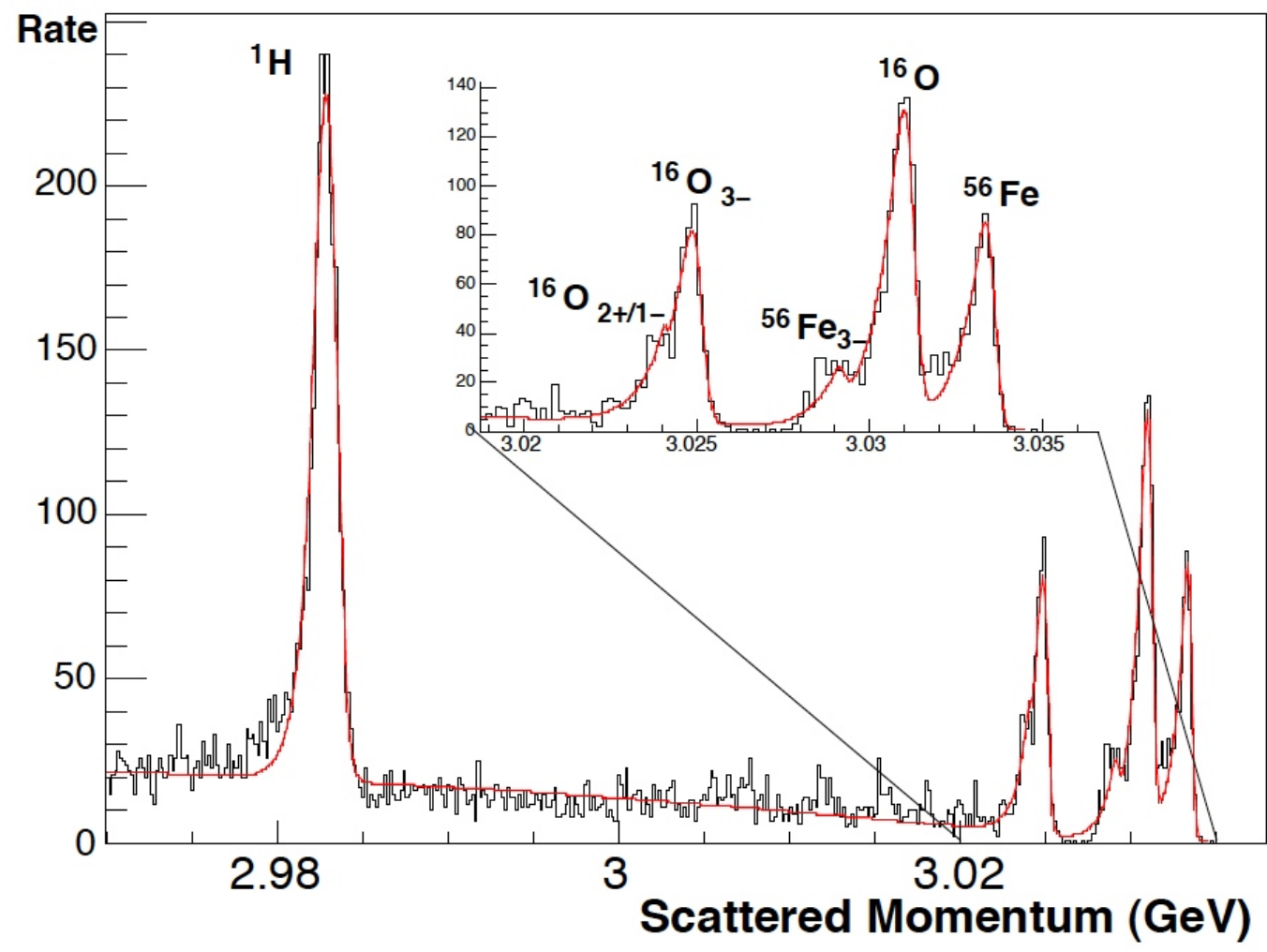

Figure 6.6: A sample fit to the reconstructed electron energy from the central sieve hole of the Right HRS. A water cell target was used for this measurement and the presented data is after kinematic corrections. 


\begin{tabular}{|l|l|l|} 
Error Source & Error (in source units) & Percent Error in $Q^{2}$ \\
\hline Scattering Angle & 0.01 degrees & $0.4 \%$ \\
HRS Momentum Scale & $5 \mathrm{MeV}$ & $0.2 \%$ \\
Beam Energy & $3 \mathrm{MeV}$ & $0.1 \%$ \\
Drifts in Time & & $0.6 \%$ \\
$\begin{array}{l}\text { Matrix Elements: } \\
\begin{array}{l}\text { At Z = } \\
\text { Z dependence }\end{array}\end{array}$ & $0.3 \%$ \\
$\begin{array}{l}\text { ADC Weighting } \\
\text { Helium } \\
\text { Hydrogen }\end{array}$ & & $0.1 \%$ \\
$\begin{array}{l}\text { Pileup (Rate Effect) } \\
\text { Helium } \\
\text { Hydrogen }\end{array}$ & & $0.1 \%(\mathrm{He})$ \\
\hline $\begin{array}{l}\text { Total Systematic Error } \\
\text { Helium } \\
\text { Hydrogen }\end{array}$ & & $0.5 \%(\mathrm{H})$ \\
Statistical Error & & $0.3 \%(\mathrm{He})$ \\
\hline $\begin{array}{l}\text { TOTAL ERROR } \\
\text { Helium } \\
\text { Hydrogen }\end{array}$ & & $0.1 \%(\mathrm{H})$ \\
\hline & & $0.9 \%(\mathrm{He})$ \\
\hline
\end{tabular}

Table 6.6: Systematic Errors in $Q^{2}$

weighted average here was $6.074^{\circ}$.

The $Q^{2}$ distribution is measured at a current of $\sim 0.5 \mu \mathrm{A}$ to keep the rate $\leq 300 \mathrm{kHz}$ and avoid pileup at the VDC's. The reconstructed $Q^{2}$ was weighted by the detector's ADC value to take account of the weighting from the integrated detector signal. The ADC weighted $Q^{2}$ for the 2005 run is displayed in Table 6.7.

The UMass $Q^{2}$ profile scanner was built to verify that the $Q^{2}$ distribution at production currents $(\sim 35$ to $55 \mu \mathrm{A})$ matched the measured distribution at low currents. Frequent $Q^{2}$ scans were taken to measure drifts in $Q^{2}$ over time; a sample $Q^{2}$ scanner distribution for 

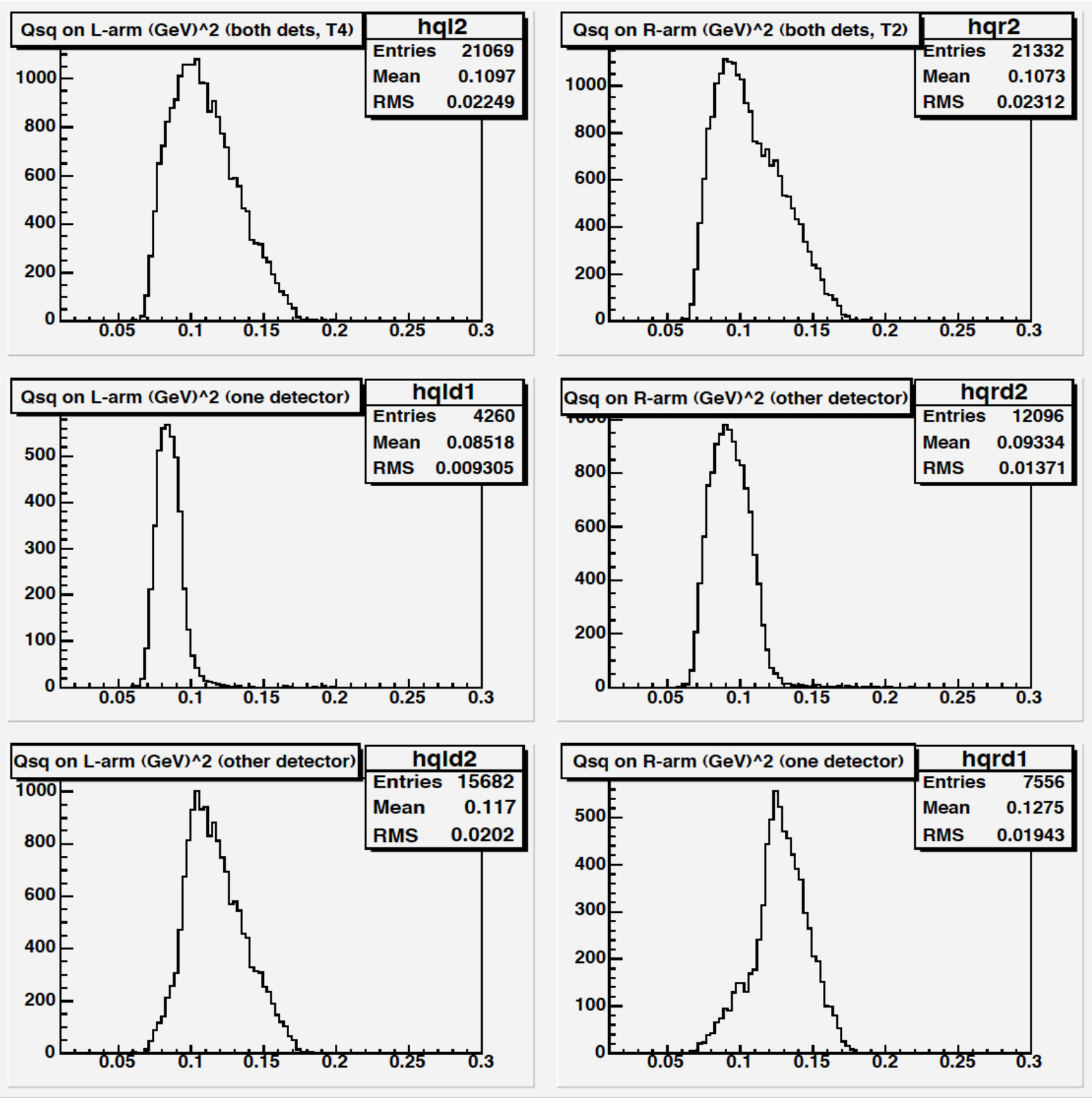

Figure 6.7: Typical 2005 Hydrogen $Q^{2}$ run on the Left and Right HRS (top row) and the individual detector segments (bottom plots). These plots are not weighted by ADC. 


\begin{tabular}{|c|c|c|}
\hline & Helium Run & Hydrogen Run \\
\hline Left Arm $Q^{2}$ & $0.07829 \pm 0.0007(0.9 \%)$ & $0.1107 \pm 0.0011(1.0 \%)$ \\
\hline Right Arm $Q^{2}$ & $0.07625 \pm 0.0007(0.9 \%)$ & $0.1072 \pm 0.0011(1.0 \%)$ \\
\hline Weighted $Q^{2}$ (Both Arms) & 0.07725 & 0.1089 \\
\hline
\end{tabular}

Table 6.7: ADC weighted $Q^{2}$ for the 2005 runs of HAPPEX-H and -He. The $Q^{2}$ numbers are in units of $(\mathrm{GeV})^{2}$
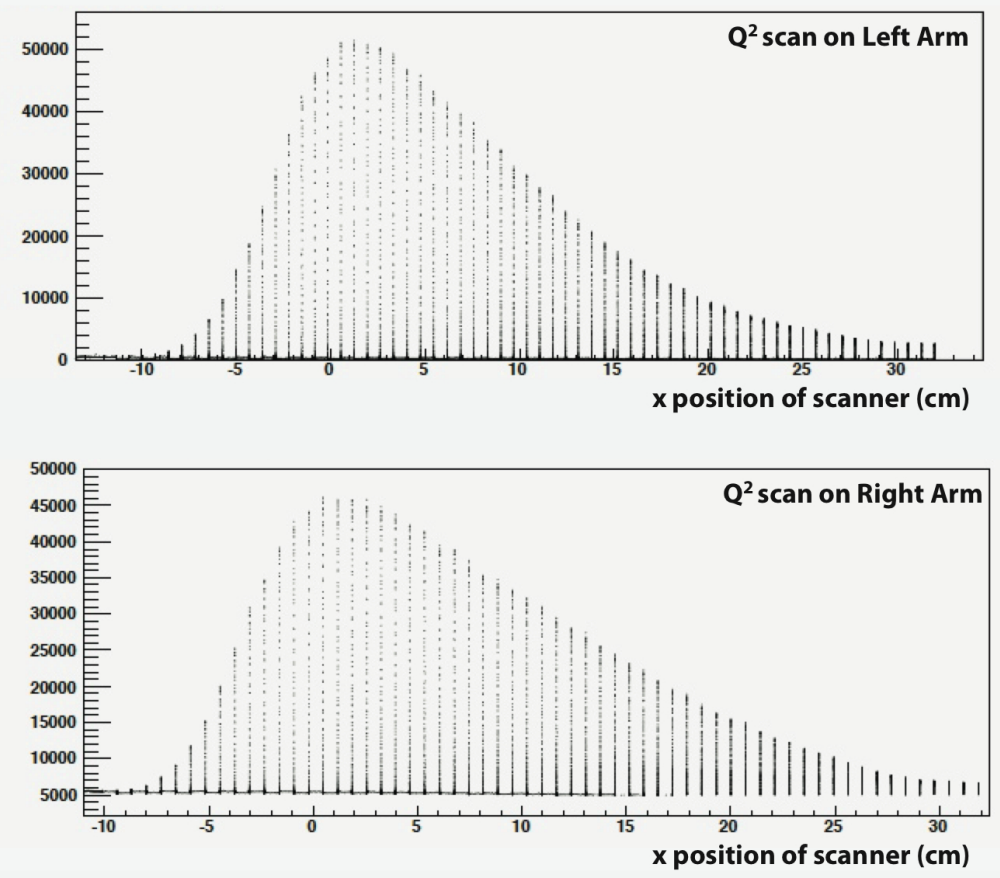

Figure 6.8: A typical $Q^{2}$ scanner run for both the left and right arms. The $x$ scale is in $\mathrm{cm}$ with an arbitrary zero, and the $y$ scale is the normalized scanner signal in ADC channels.

both left and right detectors is shown in Figure 6.7.

\subsection{Beam Polarization}

Measurement of the electron beam's polarization with the Compton Polarimeter allowed a non-invasive and continuous measurement necessary for normalizing the corrected asymmetry. The ${ }^{4} \mathrm{He}$ data had some difficulty with this technique due to the lower beam energy, 


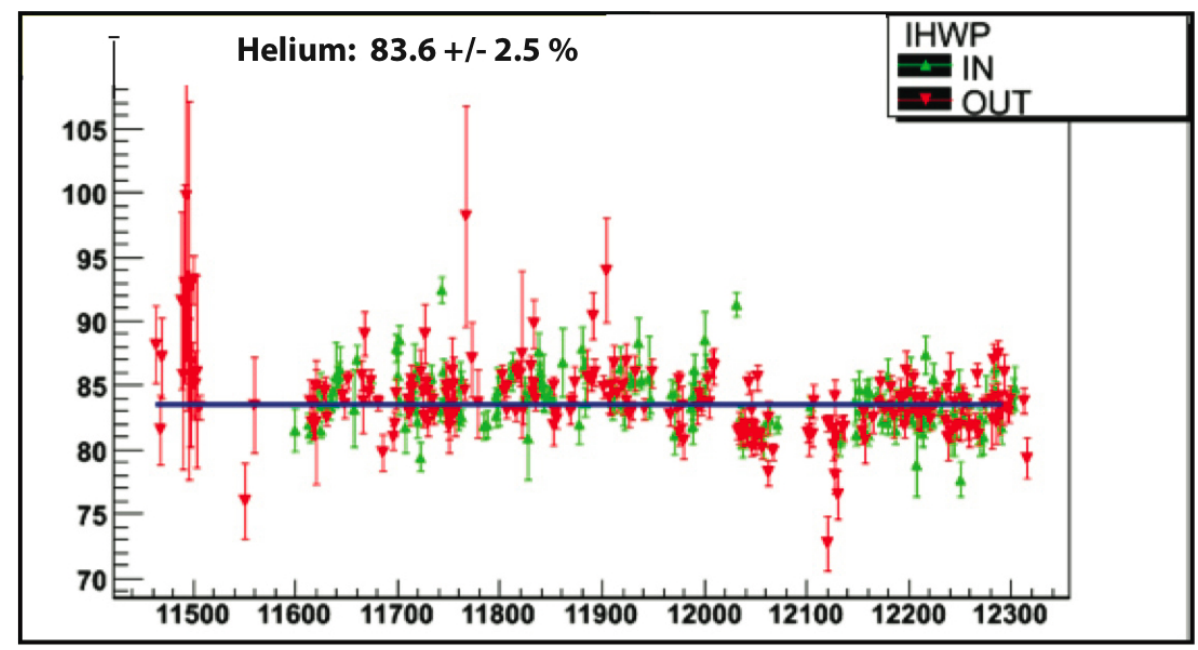

Figure 6.9: Analyzed Compton Polarimeter electron data for the 2005 HAPPEX-He run. Data in green is IHWP IN and red is IHWP OUT.

but the uncertainty remains near the $2.5 \%$ level due to confirmation to better than $1 \%$ with the Moller Polarimeter and agreement with the online photon analysis. Figures 6.9 and 6.10 display the analyzed electron data for the ${ }^{4} \mathrm{He}$ and ${ }^{1} \mathrm{H}$ run, respectively.

The beam polarization results were

$$
\begin{gathered}
P_{H e}=83.6 \pm 2.5 \% \\
P_{H}=86.3 \pm 2.0 \% .
\end{gathered}
$$

These numbers need to be divided by the laser's DOCP of 0.995 to arrive at the final numbers.

\subsection{Backgrounds}

The use of integrated signals in an asymmetry measurement presents the challenge of estimating the background contamination. For these experiments, dominant factors in this 


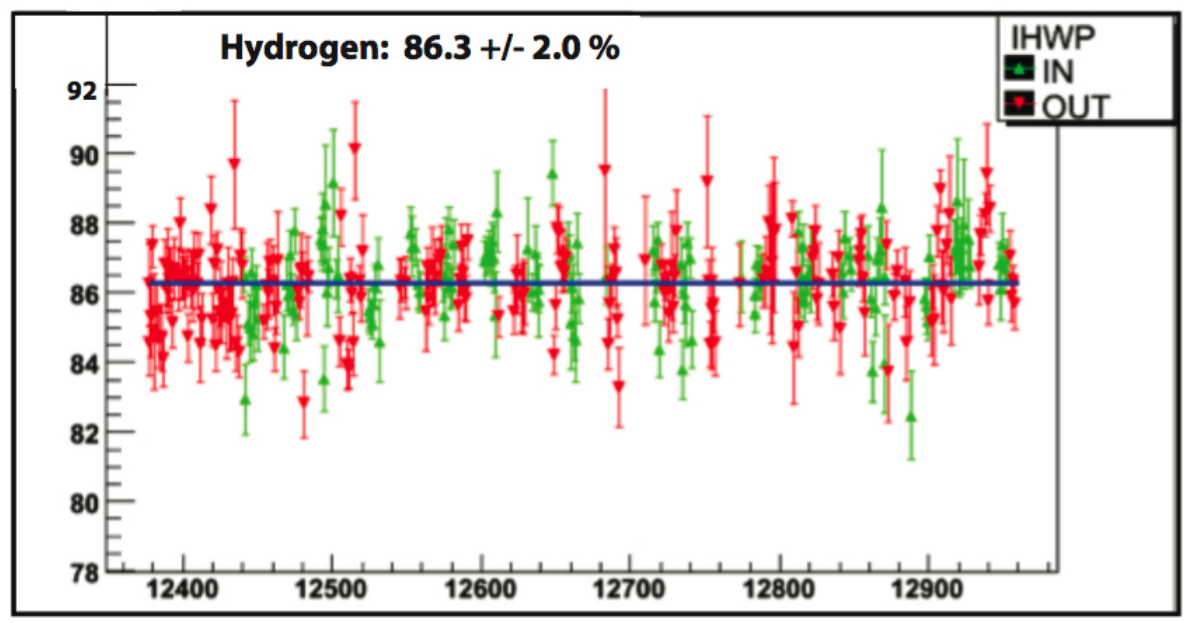

Figure 6.10: Analyzed Compton Polarimeter electron data for the 2005 HAPPEX-H run. Data in green is IHWP IN and red is IHWP OUT.

estimation included quasielastic scattering from the ${ }^{4}$ He nucleus, quasielastic scattering from the aluminum target windows, rescattering of the electron, and scattering off the poletip of the spectrometer magnet. All will be discussed in this section.

\section{5 ${ }^{4}$ He Quasielastic Scattering}

One significant contribution to ${ }^{4} \mathrm{He}$ background asymmetry for the ${ }^{4} \mathrm{He}$ experiment was quasielastic scattering from the nucleus. A minimum of $19.724 \mathrm{MeV}$ must be lost in order to do anything other than elastically scatter from ${ }^{4} \mathrm{He}$, making this the quasielastic threshold [82]. The momentum spectrum acquired with the spectrometer in "counting mode" is displayed in Figure 6.1 where the quasielastic events rising above the radiative tail appear near this threshold.

The dispersion of the dipole in the high resolution spectrometers permits kinematic separation at the target to be mapped into spatial separation at the focal plane. The HAPPEX detector could then be oriented to maximize the ratio of the elastic peak to the inelastic 


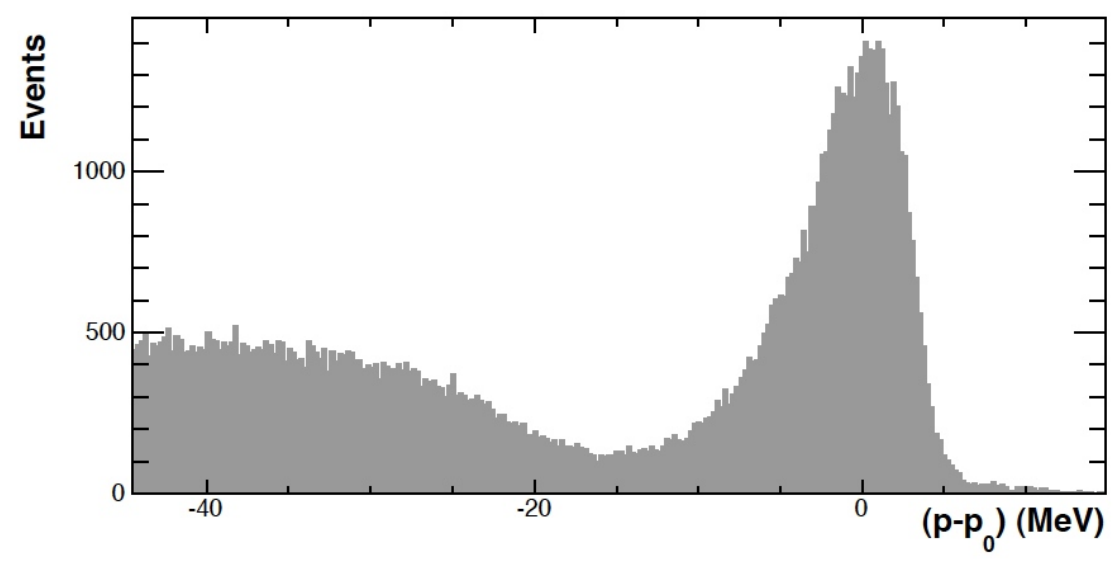

Figure 6.11: The difference of the measured momentum $p$ from the central spectrometer momentum $\left(p_{o}\right)$ from ${ }^{4} \mathrm{He}$. The rise of the quasielastic scattering that dominates the spectrum at low momenta begins between -15 and $-20 \mathrm{MeV}$.

background. The fraction of the quasielastic background was estimated by locating its rise near the low-momentum edge of the HAPPEX detector.

\section{Detector Orientation}

The HAPPEX detectors were installed in the HRS focal planes, about 0.5 meters over the VDC's and centered on the expected central ray of the elastically scattered electrons. The first step in this analysis was finding the angles of the HAPPEX detector with respect to the VDC's so that the focal plane coordinates could be rotated by these angles into the HAPPEX detector coordinate system. The resulting momentum distribution makes possible estimations of background in the detector acceptance as data can then be presented along the detector's dispersive coordinate. The red lines in Figure 6.12 are drawn at angles -32 and +35 degrees for the left arm and right arm, respectively, displaying the angle of the HAPPEX detector with respect to the VDC's. 

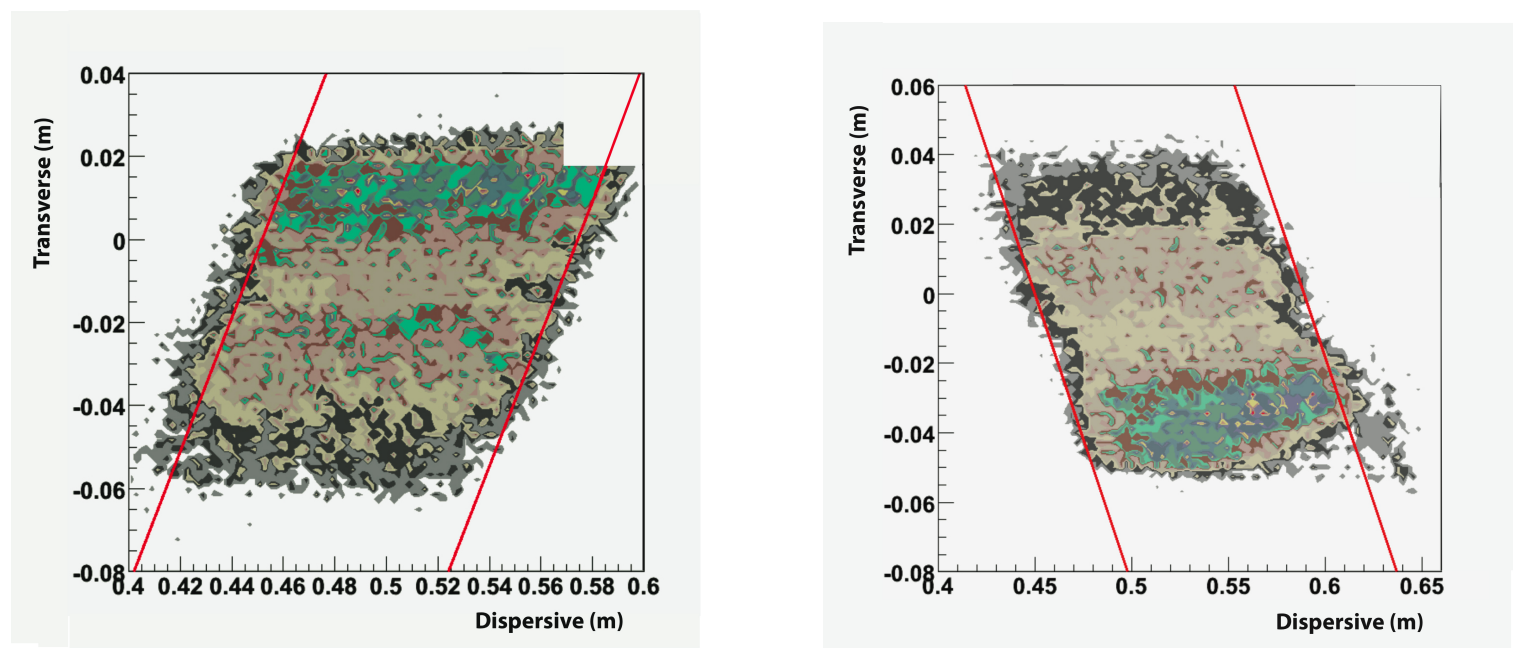

Figure 6.12: Transverse vs. Dispersive HAPPEX detector data collected from a $24 \mathrm{~cm}$ Carbon target. The left and right arm data are displayed in the left and right plots, respectively. The red lines in the plots represent the angles of the HAPPEX detectors with respect to the focal plane coordinate system.

\section{S0 Detector}

During the 2004 run, a trigger bias was identified between the HAPPEX and S2 detectors. S2 was installed above the HAPPEX detector and it was hoped that the triggers on both detectors could be used to view the entire event distribution over the whole focal plane. But efficiency differences between the detectors made estimation of the quasielastic background more difficult, potentially raising the systematic error in the measurement. A new detector called S0 was constructed to provide an unbiased trigger over the entire focal plane. S0 allowed for clearer kinematic separation between inelastic and elastic events as well as better elastic peak alignment on the HAPPEX detector [48].

\section{"Sliding" Momentum Cut}

For the 2004 quasielastic fraction estimate, a linear falloff was assumed for the quasielastic process [48] since there is a definite energy threshold below which a nucleus will not break 
apart $(-19.724 \mathrm{MeV})$. This linear model is represented by the green line in Figure 6.13. The fraction lying under the green line but within the detector's acceptance was assumed to be the upper limit of the quasielastic background as there must be some contribution to this fraction from the radiative tail. The limitations of this method became obvious when a low-momentum quasielastic tail was discovered in the centre of the detector's acceptance (Figure 6.14). This tail revealed some low momemtum events near the center of the detector's acceptance, events that would not be included in the simple falloff model for quasielastic scattering. An idea comparing the measured energy of the scattered electron $E_{\text {meas }}$ to its expected calculated energy $E^{\prime}$ based on the spectrometer's scattering angle allowed for the inclusion of the events in this low momentum tail, and hence a better estimate for the quasielastic background. Figure 6.13 also displays that there are more events triggering S0 with an $\mathrm{ADC}$ value (proportional to energy deposited in the detector from a single electron) greater than 600 (purple line) than those that only trigger the HAPPEX detector (red line). This means some electrons are hitting the detector but not triggering as if they did. For this reason, the S0 trigger with the ADC cut was used to determine what hit the detector and what did not.

Reprinting Eqn. 20 from section 6.2 on the $Q^{2}$ measurement,

$$
E^{\prime}+\epsilon^{\prime}=\frac{E_{o}-\epsilon_{o}-\frac{1}{2 m}\left(m^{* 2}-m^{2}\right)}{1+\left(E_{o}-\epsilon_{o}\right) / m(1-\cos \theta)}
$$

it is common to set $\epsilon^{\prime}=\epsilon_{o}$ and assign each half the energy loss due to ionization energy loss. We will do that here while calculating the energy loss $d E$ from the Bethe-Block equation and also set $m^{*}=m$. This leaves us with an expression for calculating the energy of a 


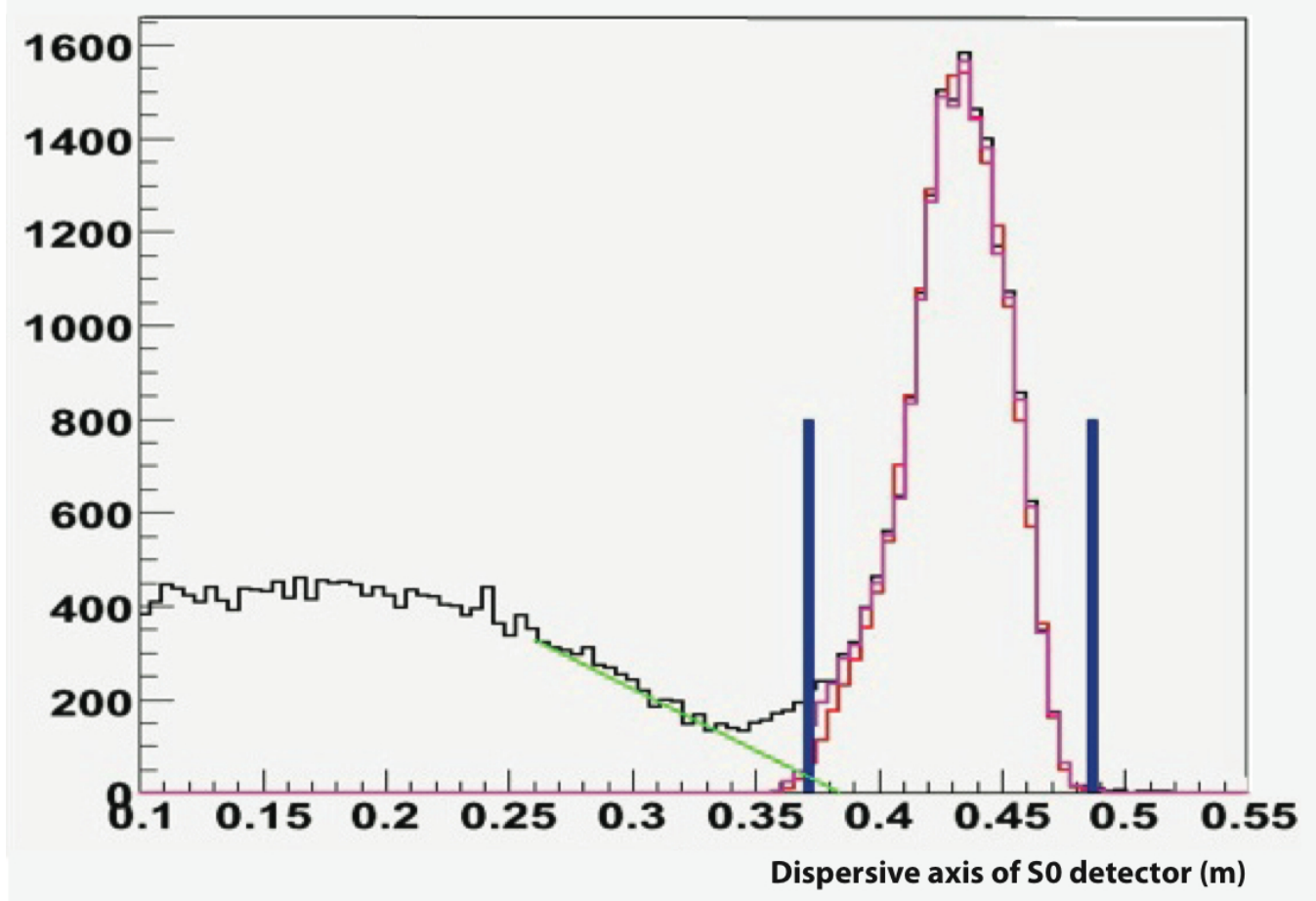

Figure 6.13: A ${ }^{4}$ He momentum distribution in the detector's dispersive axis in rotated (-32 degrees) coordinates. The green line is the linear falloff model for quasielastic scattering, the black is the S0 trigger, the purple is the S0 trigger for events with $\mathrm{ADC}$ values greater than 600, the red line is the HAPPEX detector trigger, and the blue lines are the HAPPEX detector edges.

scattered electron:

$$
E^{\prime}+\epsilon^{\prime}=\frac{E_{o}+\epsilon_{o}}{1+\frac{E_{o}}{m}\left(1-\cos \theta_{\text {scat }}\right)}
$$

where

$$
\theta_{\text {scat }}=\arccos \left(\frac{\cos \left(\theta_{o}\right)-\phi_{t g} \sin \left(\theta_{o}\right)}{\sqrt{1+\theta_{t g}^{2}+\phi_{t g}^{2}}}\right)
$$

is the electron scattering angle with $\theta_{o}$ being the spectrometer central angle, and $\theta_{t g}$ and $\phi_{t g}$ being angles measured relative to $\theta_{o}[61]$. 


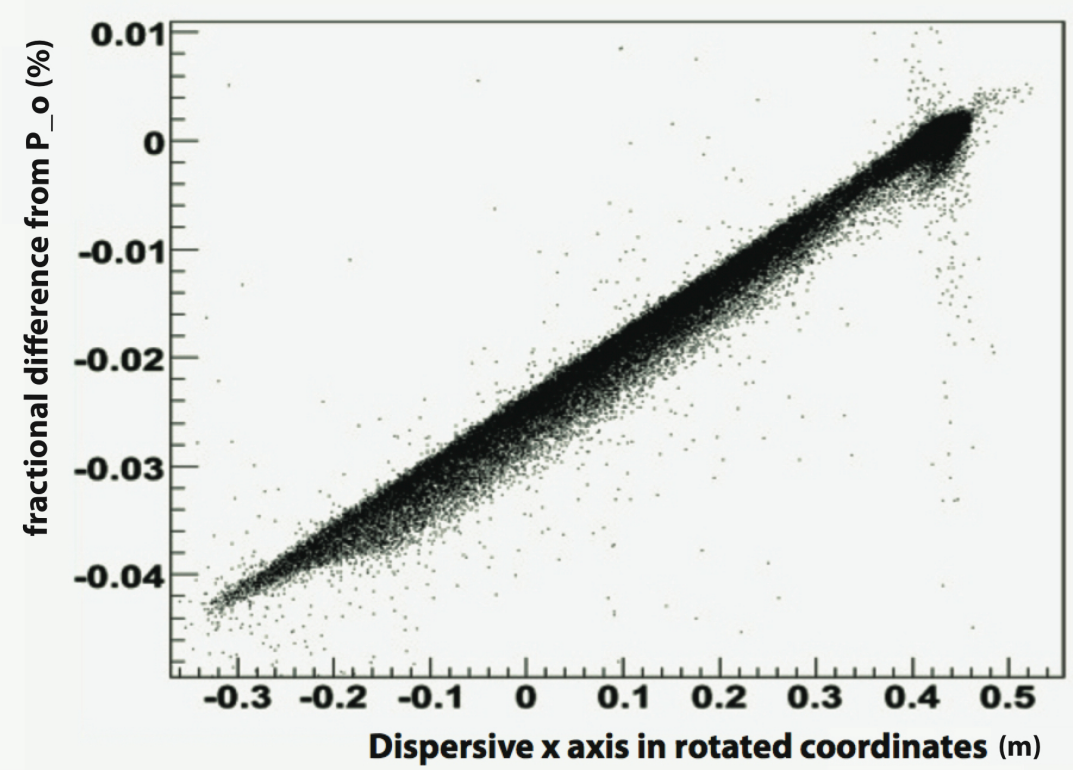

Figure 6.14: Deviation from central spectrometer momentum (\%) vs. $\mathrm{x}(\mathrm{m})$ in rotated coordinates (+35 degrees) for the right arm with data collected from the VDC's and projected up to the HAPPEX detector. The low momentum tail in the upper right portion of the figure likely contributed to the larger quasielastic fraction in the right arm versus the left.

The measured energy of a scattered electron $E_{\text {meas }}$ is

$$
E_{\text {meas }}=P_{o}+d p
$$

where $P_{o}$ is the spectrometer's central angle momentum and $d p$ is the difference of an electron's measured momentum from this central angle momentum, $P_{o}$. The energy of a scattered electron $E^{\prime}$ determined by scattering angle will differ from $E_{\text {meas }}$ due to the precision of the spectrometer's angles and spectrometer calibration. Thus, it is necessary to develop an offset to bring the difference between what is measured ( $\left.E_{\text {meas }}\right)$ and what is 
calculated $\left(\left\langle E^{\prime}\right\rangle\right)$ to zero. This offset was simply called delta $(\Delta)$ and is equal to

$$
\Delta=\left\langle E^{\prime}\right\rangle-P_{o}-\langle d p\rangle
$$

As previously stated, no quasielastic scattering can occur until until an electron loses a minimum of $19.724 \mathrm{MeV}$ [82], making the needed difference between measured and calculated the same amount. However, due to inaccuracies with spectrometer angles and calibration and potentially target ionization loss, a quasielastic scattering event could occur with an energy difference of less than $19.724 \mathrm{MeV}$. For this reason, we decided that an electron would have to lose only more than $15 \mathrm{MeV}$ to scatter quasielastically, making

$$
E_{\text {diff }}=E_{\text {meas }}+\Delta-E^{\prime}<-15 \mathrm{MeV}
$$

the cut applied for each individual electron. Since we are adjusting for each electron the difference between the measured and calculated energy with the quantity $\Delta$, this "sliding" of the momentum found it's way into the name of the cut we applied. The cut is still -15 $\mathrm{MeV}$, but some electrons can slide into or out of this cut based on their value of $\Delta$.

The $E_{\text {diff }}$ spectrum is displayed in Figure 6.15. The data in Figure 6.15 is from a ${ }^{4} \mathrm{He}$ "counting mode" spectrometer run (Run No. 2271) with cuts placed on the reconstructed target variables. Accepted particle tracks were also chosen to reduce multiplicity: 1-track events with 1 cluster and $<8$ hits per VDC plane.

Another factor in this analysis were the differences in the optics between the left and right spectrometer arms. Figure 6.16 shows the in-plane scattering angle for both the left 

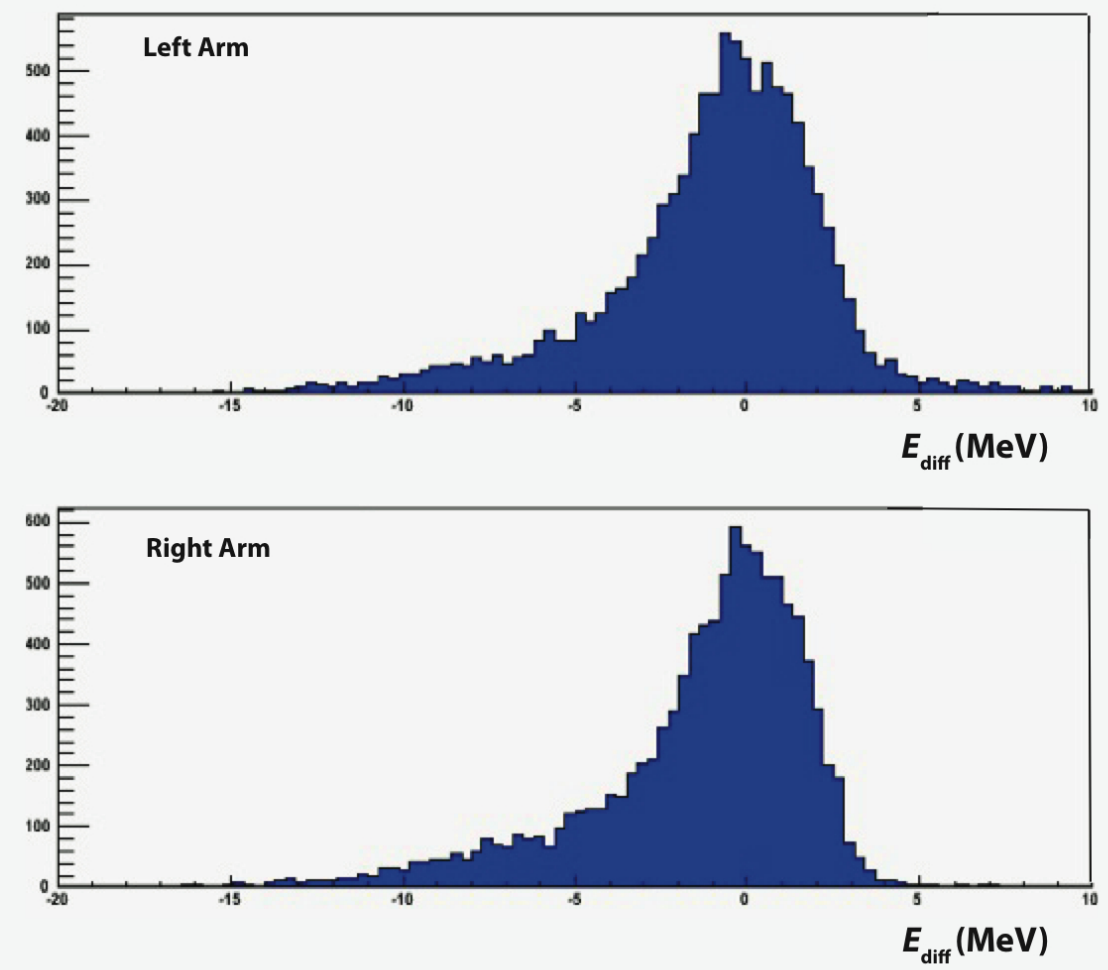

Figure 6.15: $E_{\text {diff }}$ spectrum for ${ }^{4} \mathrm{He}$ "counting mode" spectrometer run 2271.

(blue) and right (red) arms. They show sharp inner edges on both arms and an unexplained shift between them. Figures 6.17 and 6.18 show both the sharp edge on the left arm, and less of one on the right; there is also evidence of a more diffuse spectrum on the right compared to the left. There was never much of an explanation for these differences, but they may be the reason behind a magnitude of difference in the quasielastic background fraction between the two arms.

Using the "sliding" momentum cut and marking as quasielastic those events losing more than $15 \mathrm{MeV}$ upon scattering, the upper estimate on the quasielastic background was estimated to be $0.28 \pm 0.14 \%$ and $0.038 \pm 0.019 \%$ for the right and left arms, respectively. The $50 \%$ errors placed on these estimates reflect the possibility that as much as half this 


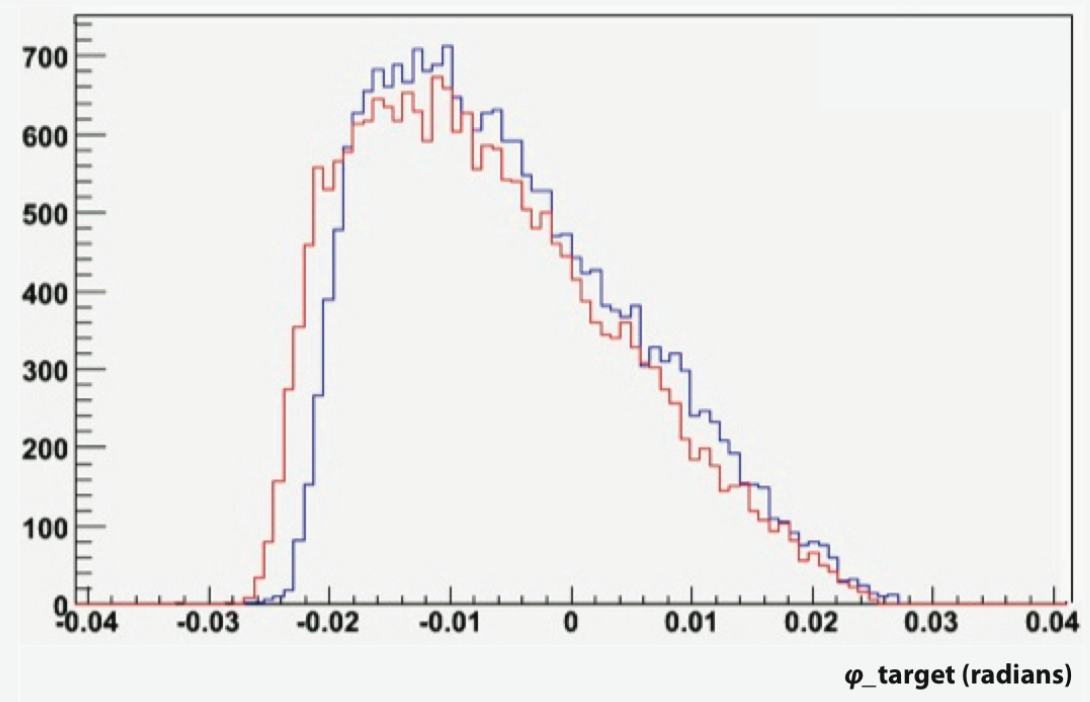

Figure 6.16: The spectrum of the in-plane scattering angle for the left (blue) and right (red) arms. The important feature to notice is the wider distribution on the right and the shift of the spectrums between the two arms.
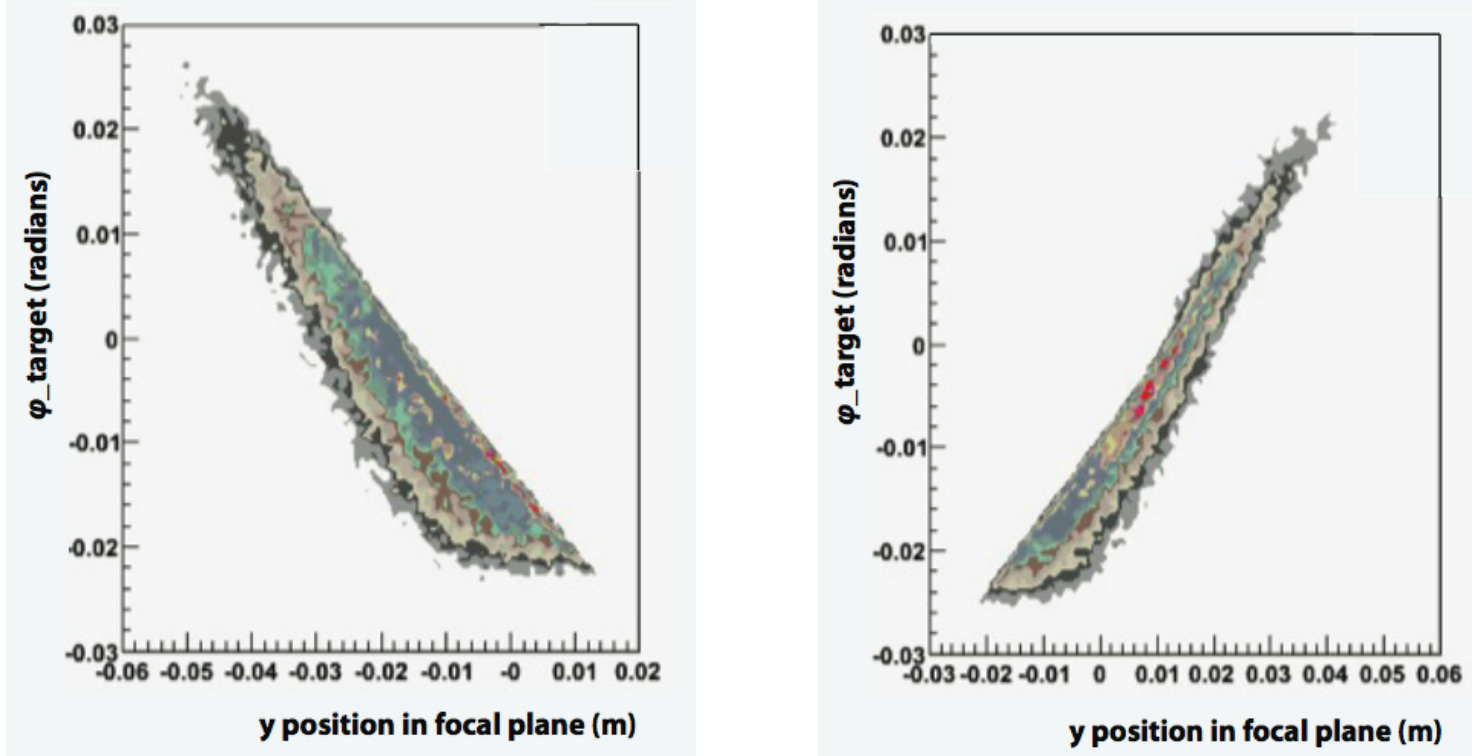

Figure 6.17: Target $\phi$ vs. focal-plane y with the left and right arms located on the left and right, respectively. The important difference to notice between the two arms is the sharper edge on the left arm and a slightly tighter correlation between the two variables on the right. 

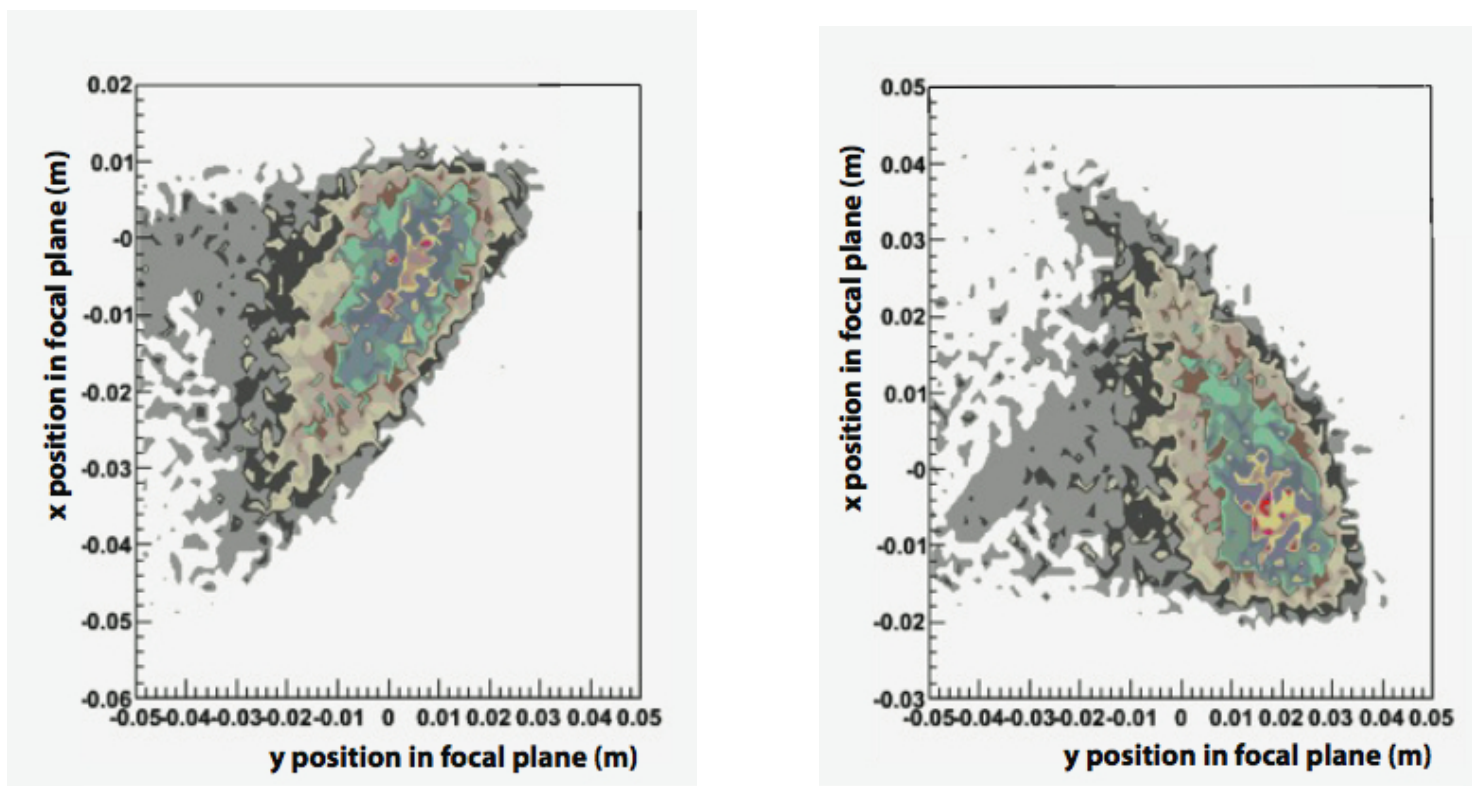

Figure 6.18: Focal plane $\mathrm{x}$ vs. $\mathrm{y}$ in rotated coordinates ( -32 degrees on the left and +35 on the right) with the left and right arm data located in the left and right plots, respectively. The difference to note here is the more diffuse spectrum on the right compared to the left.

upper limit may be due to radiative tail contribution. The $\Delta$ measurements for left and right were -0.06 and $3.51 \mathrm{MeV}$, respectively, and Figures 6.19 and 6.20 display on a log scale the x-rotated coordinates at the HAPPEX detector with the quasielastic events $(<-15$ $\mathrm{MeV}$ ) displayed in red. Ultimately, the error bar on the weighted average of the quasielastic fraction was chosen to contain the upper limit with the final fraction for both arms being $0.15 \pm 0.15 \%$.

\section{Asymmetry}

The parity-violating asymmetry for quasielastic scattering from a ${ }^{4} \mathrm{He}$ nucleus was calculated using a previously outlined method [26]. The asymmetry was calculated to be $-1.5 \pm 10.0$ ppm where the $600 \%$ error is included to account for a possible neutron/proton bias near the quasielastic scattering threshold. 


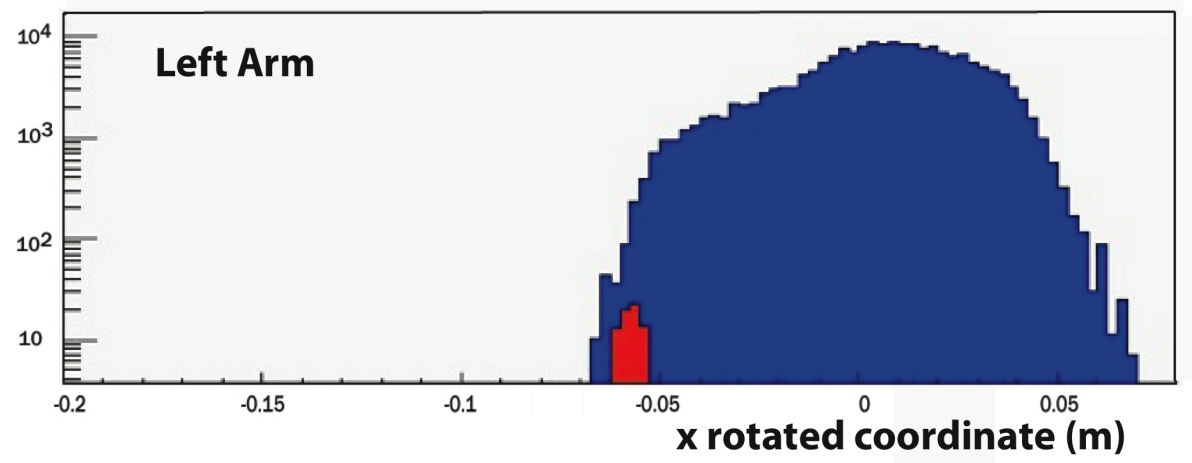

Figure 6.19: A log plot of the x-rotated coordinate at the left HAPPEX detector. Events deemed quasielastic $(<-15 \mathrm{MeV})$ are displayed in red and all others in blue. The plot is weighted by ADC over pedestal.

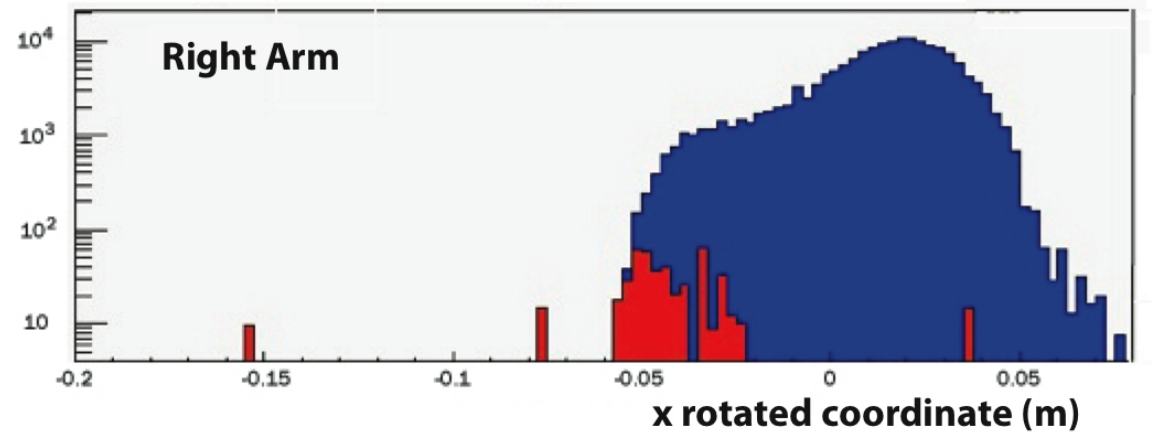

Figure 6.20: A log plot of the x-rotated coordinate at the right HAPPEX detector. Events deemed quasielastic $(<-15 \mathrm{MeV})$ are displayed in red and all others in blue. The plot is weighted by ADC over pedestal. 


\subsubsection{Aluminum Target Windows}

The background fraction due to quasielastic scattering from the aluminum end-windows of the target was estimated from both integrating and counting mode runs on a dummy target, two aluminum foils separated by 20 inches. The background fraction due to quasielastic scattering from Aluminum in the integrated detector signal was determined from the relation

$$
f_{\mathrm{Al}}=x_{t} \frac{D_{\mathrm{Al}}}{D_{4} \text { He or H }}
$$

where $x_{t}$ is the ratio of the production target window thickness to that of the dummy target foils, and $D_{\mathrm{Al}}$ and $D_{4} \mathrm{He}$ or $\mathrm{H}$ are either the rates of flux or integrated signals from the Aluminum dummy and ${ }^{4} \mathrm{He}$ or $\mathrm{H}$ production target cell, respectively. Discussions with Jefferson Lab staff about precisions of ${ }^{4} \mathrm{He}$ and $\mathrm{H}$ target cell measurements put the systematic errors here at $10 \%$ and $30 \%$, respectively. The tabulated $x_{t}$ factors were 0.278 \pm 0.03 for ${ }^{4} \mathrm{He}$ and $0.125 \pm 0.038$ for hydrogen. Integrating mode was used for both ${ }^{4} \mathrm{He}$ and $\mathrm{H}$ with counting mode providing a needed check. The weighted 2 -arm fraction for ${ }^{4} \mathrm{He}$ was $0.018 \pm 0.002$ with the weighted fraction for $\mathrm{H}$ being $0.0076 \pm 0.0025$.. The calculated asymmetry [26] for quasielastic scattering from $\mathrm{Al}$ in the ${ }^{4} \mathrm{He}$ case was $-1.5 \pm 1.5 \mathrm{ppm}$ and $-1.9 \pm 1.9 \mathrm{ppm}$ in the $\mathrm{H}$ case. The $100 \%$ uncertainty in both cases is to account for possible resonance structure, elastic contributions, and changing kinematics.

\subsubsection{Rescattering in the Spectrometer}

Another source of background contamination comes from inelastic scattering off ${ }^{4} \mathrm{He}$ or $\mathrm{H}$ nuclei, and then the rescattering of those electrons inside the spectrometer. Most of 
this background consists of low-energy charged or neutral particles that contribute to the HAPPEX detector's integrated signal. A model was developed to measure this background given by [48]

$$
f_{\mathrm{rs}}=\int_{E_{\min }}^{E_{\max }} P_{\mathrm{rs}}(E) R(E) d E
$$

where the probability of rescatter $P_{r s}$ in the spectrometer is weighted by the energy deposited $E_{\text {dep }}$ in the detector, normalized to the deposited energy of an elastically scattered electron $E_{\mathrm{O}}$ :

$$
P_{\mathrm{rS}}(E)=(\text { rescatter probability }) \times\left(\frac{E_{\mathrm{dep}}}{E_{\mathrm{O}}}\right)
$$

and $R(E)$ is the ratio of the inelastic to the elastic cross section:

$$
R(E)=\frac{\left(\frac{d \sigma}{d \Omega d E}\right)_{\text {inelastic }}}{\left(\frac{d \sigma}{d \Omega}\right)_{\text {elastic }}}
$$

The limits on the integral in Equation 6.31 are from the inelastic energy threshold $E_{\text {min }}$ to the maximum inelastically scattered energy that could contribute to the background fraction $\left(E_{\max }\right)$

The rescattering inside the spectrometer is measured with two momentum scans of the spectrometer's dipole. The first increases the field of the dipole magnet and sweeps the elastic peak across the focal plane. At momentum increases of 2, 4, 8, 12, 14, and 20 percent, we measured the signal size in the detector relative to the signal size of the elastic peak. Figure 6.21 displays both counting and integrating mode data for ${ }^{4} \mathrm{He}$, although only 
the later was used. The counting mode data was unreliable due to the low signal size relative to the trigger threshold.

The cross-section ratio $R(E)$ was estimated by lowering the dipole momentum to measure the inelastic spectrum entering the spectrometer during production running. These runs were combined with relative scaling to create a smooth spectrum from which $R(E)$ is measured as a function of momentum. Figure 6.22 shows the ${ }^{4}$ He inelastic spectrum created from 4 lower spectrometer momentum runs: each one normalized to the elastic peak signal to calculate $R(E)$. The gaps in the relative rates for Figure 6.21 were filled in by estimating a trend to the graph between the plotted points, as we needed data at four lower momentum settings from the inelastic spectrum.

The true rescattering probability was estimated from subtracting from the relative rates in Figure 6.21 any rescattering from other areas of the inelastic spectrum that rescatter with lower probability. This is important so that only rescattering from one area of the inelastic spectrum is included in the true probability. If this is not done, we are double counting rescattering events from one area of the inelastic spectrum in the estimation of another.

After the relative cross sections of the four sections displayed in Figure 6.22 were calculated, the relative rates adjusted into true rescattering probabilities, the corresponding sections were multiplied together (rescattering probability $\mathrm{x}$ relative cross section), then added together, and were found to contribute a background fraction to the ${ }^{4}$ He measurement of $0.25 \pm 0.15 \%$. The uncertainty was likely dominated by the limitation of this analysis being only performed on the left arm and any differences on the right are unknown. The ${ }^{4}$ He quasielastic asymmetry was used with a $200 \%$ error to consider various kinematics and 
contributions from inelastic events. The asymmetry used here was the expected QE PV asymmetry $-1.5 \pm 3 \mathrm{ppm}$.

The same procedure was followed with the hydrogen data but with a much smaller rescattering fraction. Hydrogen has a much smaller radiative tail and a much less messy inelastic spectrum to account for. It is much more like a point probe than is helium. Figure 6.24 does show a delta resonance peak at about $300 \mathrm{MeV}$ from the elastic peak. This is important because the asymmetry from scattering in the delta region is different than that from the radiative tail (same as detected hydrogen asymmetry). So, the inelastic spectrum was integrated before the elastic peak, and the tail was extrapolated into the delta region. Adding up the colored distributions minus the delta contribution resulted in a fraction of $0.07 \%$. The delta contributed $0.03 \%$. The asymmetry used for the delta region was $-9.6 \pm 100 \% \mathrm{ppm}$ and that for the radiative tail was $-1.6 \pm 50 \% \mathrm{ppm}$. The weighted average was $-3.9 \pm 3.4 \mathrm{ppm}$. We used $4 \pm 4 \mathrm{ppm}$.

\subsubsection{Poletip}

The last source of background comes from scattering from the tip of the dipole magnet (poletip) and entering the HAPPEX detector acceptance. There is an estimation needed from both the front and rear end of the dipole; there were many events scattering off of the top and bottom, but this did not matter as there is no polarized iron there. First, an estimation was needed for the number of poletip candidates which is the data outside of the dipole acceptance. Various suppression factors are estimated such as angular acceptance and the distribution of the data coming from the dipole. The polarization of iron was included $(8 \%)$, transverse analyzing power $(1 / 7)$, longitudinal analyzing power $(1 / 9)$, and energy loss 


\section{Dipole Field Scan: Relative Rate vs \% Field}

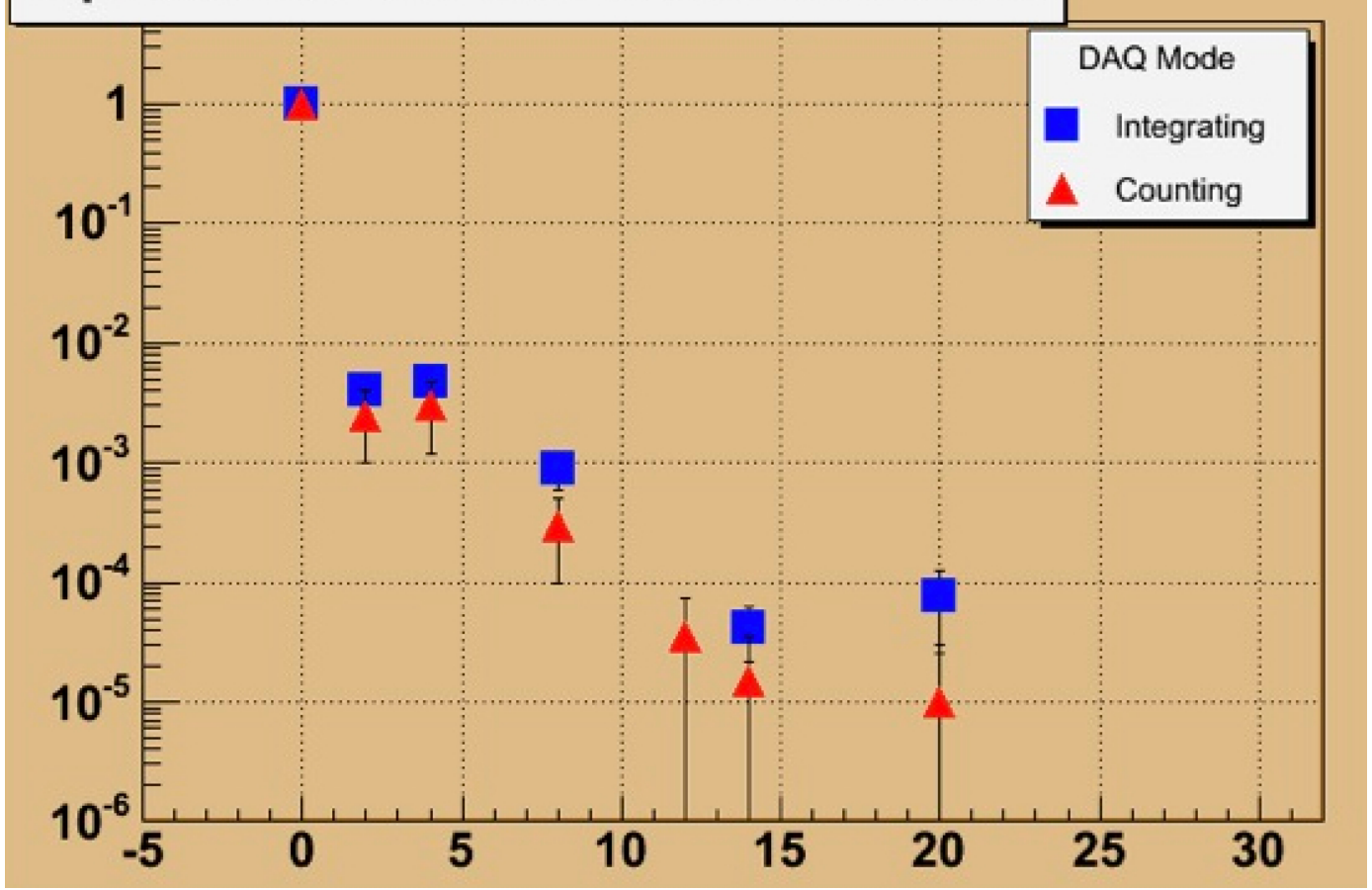

Figure 6.21: $\frac{E_{\mathrm{dep}}}{E_{\mathrm{o}}}$ for ${ }^{4} \mathrm{He}$ with $E_{\text {dep }}$ at increased momentum dipole settings of $2,4,8,12,14$, and 20 percent. All points are scaled by the energy deposited into the detector from elastically scattered electrons, $E_{o}$. 


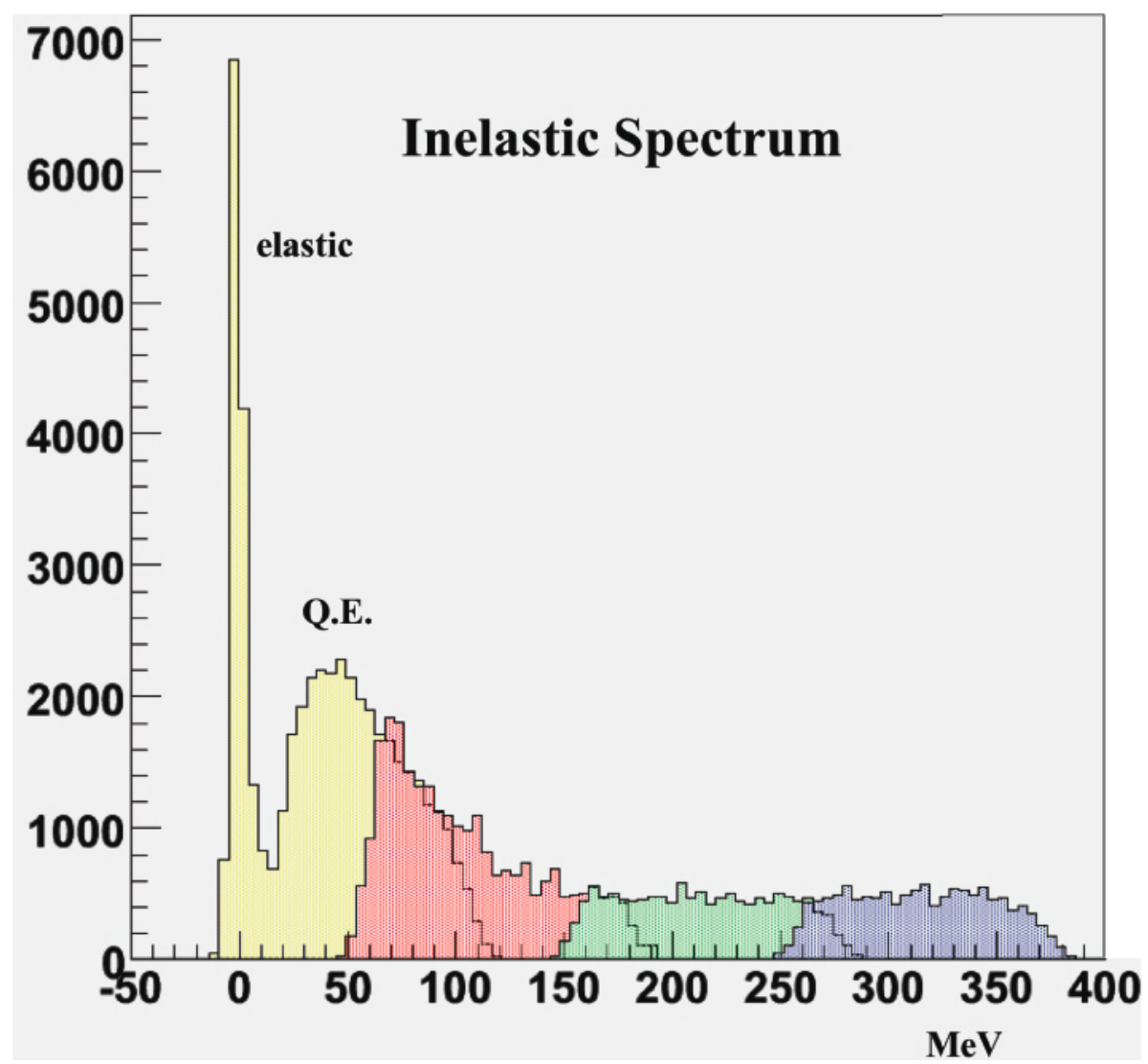

Figure 6.22: ${ }^{4} \mathrm{He}$ inelastic spectrum plotted versus deviation from the elastic peak in $\mathrm{MeV}$. The four different colors represent four different "integrating mode" spectrometer runs at lower momentum settings on the dipole. 


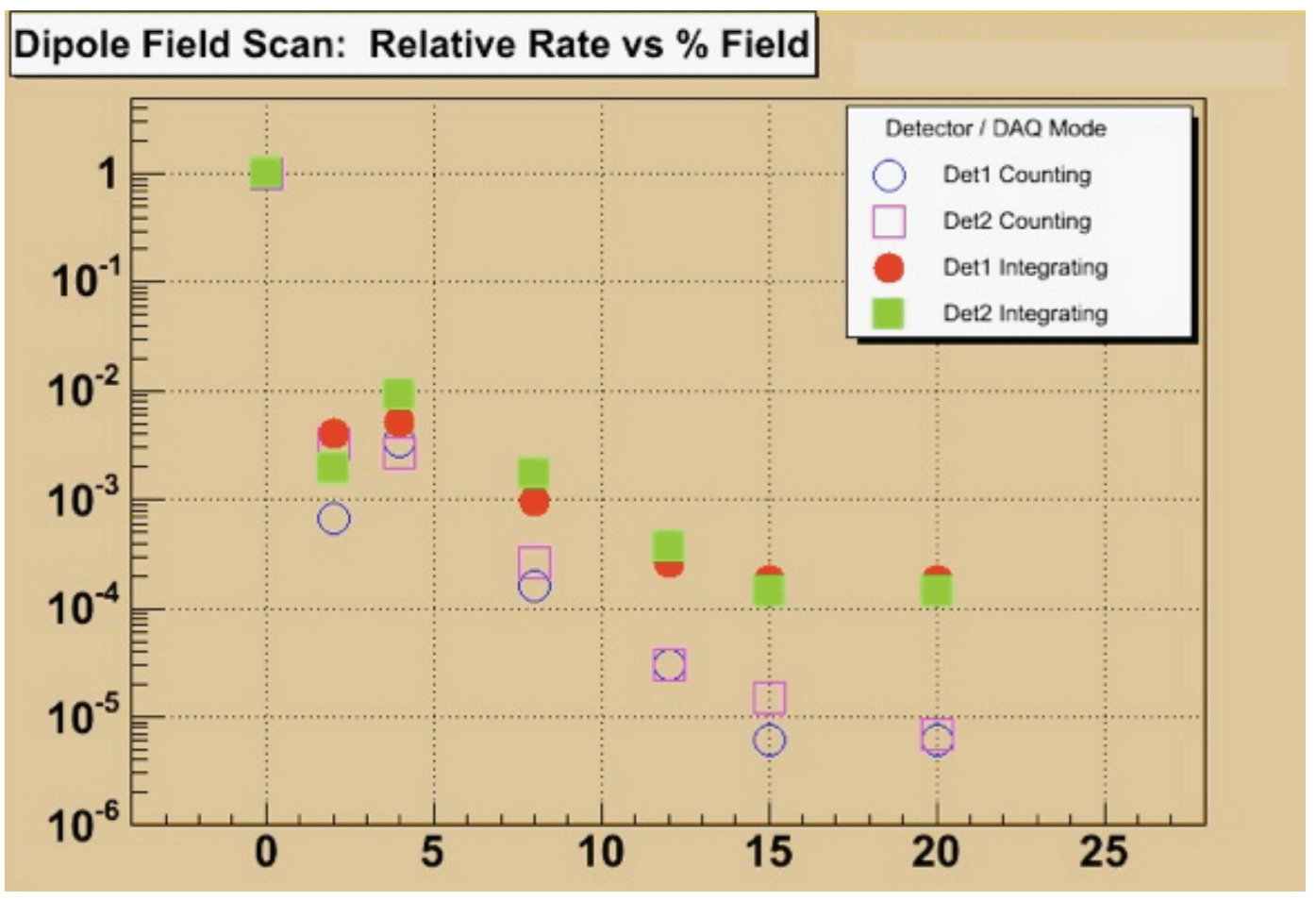

Figure 6.23: $\frac{E_{\text {dep }}}{E_{\mathrm{o}}}$ for Hydrogen with $E_{\text {dep }}$ at increased momentum dipole settings of 2, 4, 8, 12, 15 , and 20 percent. All points are scaled by the energy deposited into the detector from elastically scattered electrons, $E_{o}$ 


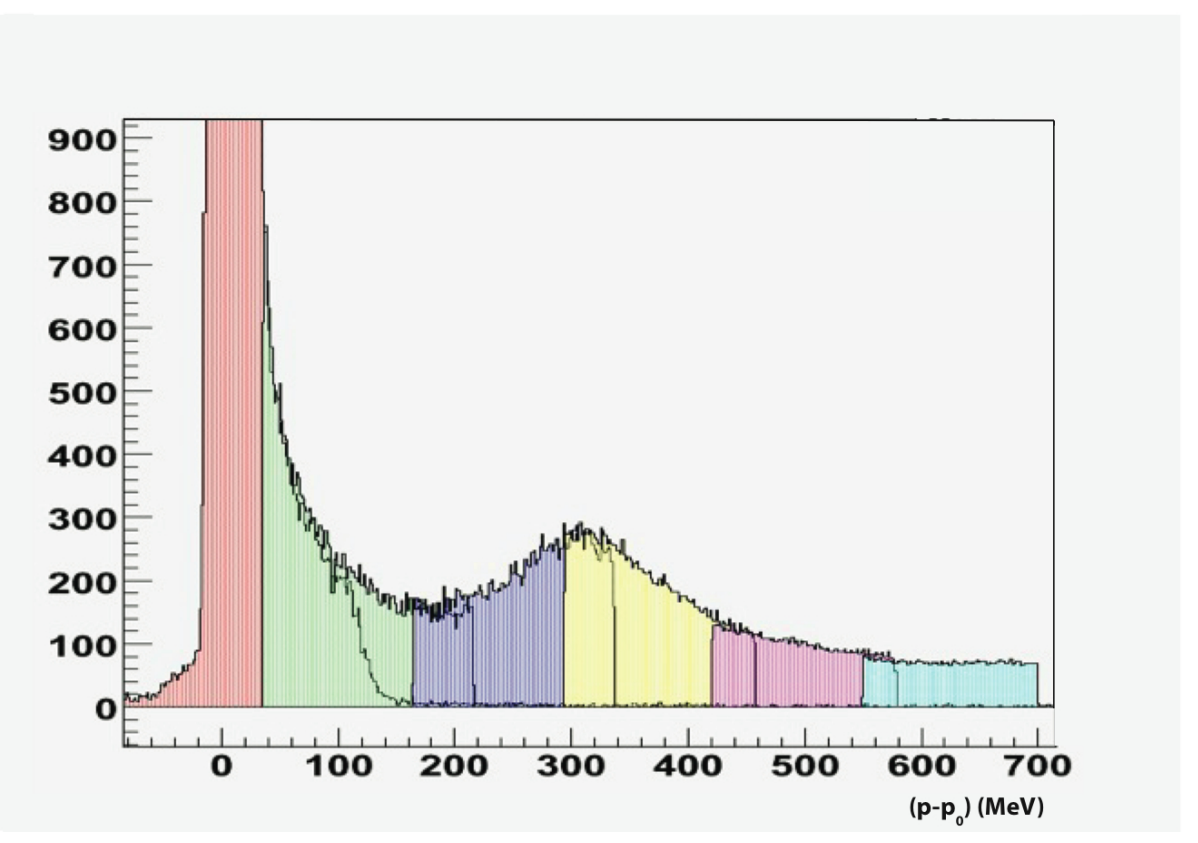

Figure 6.24: Hydrogen inelastic spectrum plotted versus deviation from the elastic peak in $\mathrm{MeV}$. The different colors represent different "integrating mode" spectrometer runs at lower momentum settings on the dipole.

(1/10 due to radiation). These factors multiply together to give an asymmetry of $1.3 \times 10^{-4}$ for the poletip. The poletip fraction is found by doing the integral for Eqn. 8 with an additional factor $P_{f}$, poletip candidate fraction, in the integrand; $P_{f}$ was found to be same for both the ${ }^{4} \mathrm{He}$ and Hydrogen data, $4 \times 10^{-4}$. The fraction from completing the integral had upper limits of $340 \mathrm{ppm}$ for ${ }^{4} \mathrm{He}$ and $240 \mathrm{ppm}$ for hydrogen. When including suppression factors of $1 / 100$ in each case, the poletip correction to the asymmetry is about $0.4 \mathrm{ppb}$ for the rear end of the poletip. When adding a $2 \mathrm{ppb}$ estimation for the front end, the estimated correction for poletip scattering is not more than $\sim 3 \mathrm{ppb}$. 


\subsection{Effective Kinematics}

The finite acceptance of the spectrometer, radiative energy losses, and multiple scattering in the target cell convolute the measured asymmetry over the range of values in the $Q^{2}$ distribution. These effects must be accounted for if the asymmetry is to be accurately represented at a single value for $Q^{2}$. A Monte Carlo simulation (called gener_cone) was used to calculate the the parity-violating asymmetry at the effective $Q^{2}$ of the experiment. The result represents point scattering at the kinematics of that effective $Q^{2}$. In the Monte Carlo, the spectrometer acceptance and energy losses are included to reproduce the measured $Q^{2}$ distribution and ultimately calculate the average parity-violating asymmetry.

The effective kinematics factor $K$ is calculated from

$$
K=\frac{A_{P V}^{e f f}}{A_{P V}^{\text {meas }}}
$$

where $A_{P V}^{e f f}$ is the parity-violating asymmetry calculated from the experiment's effective $Q^{2}$ and $A_{P V}^{\text {meas }}$ is the parity-violating asymmetry calculated to represent the experiment's measured $Q^{2}$ distribution. The kinematic factors were found to be $K_{H e}=1.000 \pm 0.001$ and $K_{H}=0.979 \pm 0.002$.

\subsection{Physics Asymmetry}

The physics asymmetry $A_{\text {phys }}$ is calculated from

$$
A_{\mathrm{phys}}=\frac{K L}{P_{b}} \frac{A_{\mathrm{corr}}-P_{b} \sum_{i} A_{i} f_{i}}{1-\sum_{i} f i}
$$




\begin{tabular}{c|l|c|c|c}
\hline \hline Term & Description & Value & Error & Units \\
\hline$f_{1}$ & Al target QE fraction & 0.018 & 0.002 & \\
$A_{1}$ & Al target QE asymmetry & -1.5 & 1.5 & $\mathrm{ppm}$ \\
$f_{2}$ & He-QE fraction & 0.0015 & 0.0015 & \\
$A_{2}$ & He-QE asymmetry & -1.5 & 10 & $\mathrm{ppm}$ \\
$f_{3}$ & He rescatter fraction & 0.0025 & 0.0015 & \\
$A_{3}$ & He rescatter asymmetry & -1.5 & 3 & $\mathrm{ppm}$ \\
$P_{b}$ & Beam Polarization & 0.836 & 0.03 & \\
$L$ & Linearity (BCM) & 1 & 0.02 & \\
$L$ & Linearity (Det) & 1 & 0.01 & \\
$K$ & Effective Kinematics & 1.0 & 0.001 & \\
\hline \hline
\end{tabular}

Table 6.8: Summary of corrections and errors for all the terms in $A_{\text {phys }}$ for the ${ }^{4} \mathrm{He}$ measurement.

\begin{tabular}{c|l|c|c|c}
\hline \hline Term & Description & Value & Error & Units \\
\hline$f_{1}$ & Al target QE fraction & 0.0076 & 0.0025 & \\
$A_{1}$ & Al target QE asymmetry & -2 & 2 & $\mathrm{ppm}$ \\
$f_{2}$ & He-QE fraction & 0.001 & 0.0005 & \\
$A_{2}$ & He-QE asymmetry & -4 & 4 & $\mathrm{ppm}$ \\
$P_{b}$ & Beam Polarization & 0.863 & 0.02 & \\
$L$ & Linearity (BCM) & 1 & 0.02 & \\
$L$ & Linearity (Det) & 1 & 0.01 & \\
$K$ & Effective Kinematics & 0.979 & 0.002 & \\
\hline \hline
\end{tabular}

Table 6.9: Summary of corrections and errors for all the terms in $A_{\text {phys }}$ for the ${ }^{1} \mathrm{H}$ measurement.

where $A_{\text {phys }}$ is formed from $A_{\text {corr }}$ by correcting for the beam polarization $P_{b}$, background fractions $f_{i}$ and the corresponding asymmetries $A_{i}$, linearity $L$, and a kinematic factor $K$.

Table 6.5 presents a summary of the various factors needed to calculate $A_{\text {phys }}$ for ${ }^{4} \mathrm{He}$, and Table 6.6 presents those needed for the ${ }^{1} \mathrm{H} A_{\text {phys }}$. The statistical error for $A_{\text {phys }}$ in both measurements was determined from the statistical error of $A_{\text {corr }}$ in each case.

After all corrections, the physics asymmetry from the ${ }^{4} \mathrm{He}$ measurement was found to be

$$
A_{\text {phys }}=-6.43 \pm 0.23(\text { stat }) \pm 0.22 \text { (syst) } \mathrm{ppm},
$$




\begin{tabular}{l|r|r}
\hline \hline Description & \multicolumn{2}{|c}{ Error Contribution (ppb) } \\
& Individual & Total \\
\hline False Asymmetry & 12 & 48 \\
$\quad$ Energy & 46 & \\
Position/Angle & 8 & \\
$A_{T}$ & & 58 \\
$\mathrm{Q}^{2}$ & & 40 \\
Backgrounds & 32 & \\
Al-QE & 18 & \\
He-QE & 14 & \\
He-QE rescatter & 0 & \\
Delta rescatter & 5 & \\
Poletip & & \\
Beam Polarization & 9 & 58 \\
Linearity & 57 & \\
BCM & & \\
Det/BCM & & \\
\hline Total Systematic Error & & \\
\hline \hline
\end{tabular}

Table 6.10: Contributions to the ${ }^{4} \mathrm{He} A_{\text {phys }}$ systematic error

and that for the ${ }^{1} \mathrm{H}$ measurement was found to be

$$
A_{\text {phys }}=-1.60 \pm 0.12 \text { (stat) } \pm 0.05 \text { (syst) ppm. }
$$

The contributions of the errors in the corrections to the final systematic error for $A_{\text {phys }}$ were obtained through standard error propagation assuming uncorrelated contributions from each source. The errors from each term were added in quadrature. The contributed systematic errors for the ${ }^{4} \mathrm{He}$ measurement are displayed in Table 6.7 with those for ${ }^{1} \mathrm{H}$ in Table 6.8 . 


\begin{tabular}{l|r|r}
\hline \hline Description & \multicolumn{2}{|c}{$\begin{array}{c}\text { Error Contribution (ppb) } \\
\text { Individual }\end{array}$} \\
\hline False Asymmetry & 0 & Total \\
$\quad$ Energy & 14 & 48 \\
Position/Angle & 4 & \\
$A_{T}$ & & \\
$\mathrm{Q}^{2}$ & 15 & 16 \\
Backgrounds & 4 & 16 \\
$\quad$ Al-QE & 3 & \\
$\quad$ Rescatter & & \\
Poletip & & 37 \\
Beam Polarization & 10 & 15 \\
Linearity & 11 & \\
BCM & & \\
$\quad$ Det/BCM & & \\
\hline Total Systematic Error & & \\
\hline \hline
\end{tabular}

Table 6.11: Contributions to the ${ }^{1} \mathrm{H} A_{\text {phys }}$ systematic error 


\section{Chapter 7}

\section{Results and Conclusions}

This thesis concludes by extracting the strange form factors from the measured asymmetry. The results will be discussed within the context of similar measurements, and an overview of future parity-violating experiments at Jefferson Lab will be provided at the end.

\section{$7.1 \quad$ Results}

The parity-violating asymmetries introduced in Chapter 2 for both ${ }^{4} \mathrm{He}$ and hydrogen must be modified from their tree-level expressions to consider electroweak radiative corrections for comparison to the measured asymmetries. The hydrogen asymmetry given in the standard model is [26]

$$
\begin{aligned}
A_{L R}= & -\frac{G_{F} Q^{2}}{4 \pi \alpha \sqrt{2}} \times\left\{\left(1+R_{V}^{p}\right)\left(1-4 \sin ^{2} \theta_{W}\right)\right. \\
& -\left(1+R_{V}^{n}\right) \frac{\epsilon G_{E}^{p \gamma} G_{E}^{n \gamma}+\tau G_{M}^{p \gamma} G_{M}^{n \gamma}}{\epsilon\left(G_{E}^{p \gamma}\right)^{2}+\tau\left(G_{M}^{p \gamma}\right)^{2}}-\left(1-R_{V}^{(0)}\right) \frac{\epsilon G_{E}^{p \gamma} G_{E}^{s}+\tau G_{M}^{p \gamma} G_{M}^{s}}{\epsilon\left(G_{E}^{p \gamma}\right)^{2}+\tau\left(G_{M}^{p \gamma}\right)^{2}} \\
& \left.-\frac{\left(1-4 \sin ^{2} \theta_{W}\right) \epsilon^{\prime} G_{M}^{p \gamma}}{\epsilon\left(G_{E}^{p \gamma}\right)^{2}+\tau\left(G_{M}^{p \gamma}\right)^{2}}\left[-2\left(1+R_{A}^{T=1}\right) G_{A}^{T=1}+\left(\sqrt{3} R_{A}^{T=0}\right) G_{A}^{T=0}\right]\right\}
\end{aligned}
$$




\begin{tabular}{|c|c|}
\hline \multicolumn{2}{|c|}{${ }^{1} \mathrm{H}$ Electroweak Radiative Corrections } \\
\hline$R_{V}^{p}$ & -0.045 \\
$R_{V}^{n}$ & -0.0118 \\
$R_{V}^{(0)}$ & 0.0117 \\
$R_{A}^{T=1}$ & $-0.173+[-0.086 \pm 0.346$ (anapole) $]$ \\
$R_{A}^{T=0}$ & $-0.253+[-0.014 \pm 0.202$ (anapole) $]$ \\
\hline
\end{tabular}

Table 7.1: Vector and axial electroweak radiative corrections, calculated using the $\overline{\mathrm{MS}}$ renormalization scheme, used in the expression for $A_{L R}$.

where

$$
\tau=\frac{Q^{2}}{4 M_{p}^{2}}, \quad \epsilon=\left[1+2(1+\tau) \tan ^{2}\left(\frac{\theta}{2}\right)\right]^{-1}, \quad \text { and } \quad \epsilon^{\prime}=\sqrt{\tau(1+\tau)\left(1-\epsilon^{2}\right)}
$$

$G_{F}$ is the Fermi coupling constant, $\alpha$ is the fine structure constant, and $\theta_{W}$ is the electroweak mixing angle. $G_{E(M)}^{p \gamma}$ is the proton electric (magnetic) form factor, $G_{E(M)}^{n \gamma}$ is the neutron electric (magnetic) form factor, and $G_{A}^{T=1(0)}$ is the isovector (isoscalar) proton axial form factor. All form factors are functions of $Q^{2}$. The $R_{V(A)}$ terms are the radiative corrections of the neutral weak current and are independent of $Q^{2}$. Their values are summarized in Table $7.1[84,85]$.

The minimum subtraction $(\overline{\mathrm{MS}})$ renormalization scheme is used to calculate the electroweak radiative corrections where $\sin ^{2} \theta_{W} \equiv \sin ^{2} \hat{\theta}\left(M_{Z}\right)=0.2312$ [84]. There are two parts to the $R_{A}^{T=0(1)}$ factors, the electroweak corrections given by [84] and the anapole contribution (a parity-violating coupling of the photon to the proton) calculated by [85]. The electromagnetic corrections are negligible due to two factors. Firstly, the momentum acceptance $\delta p / p<3 \%$, implying that the emitted photons are relatively soft, and secondly, there is eseentially no spin dependence in the emission of these photons. Thus, the electromag- 


\begin{tabular}{|c|c|}
\hline \multicolumn{2}{|c|}{${ }^{4}$ He Electroweak Coupling Constants } \\
\hline$\rho^{\prime}$ & 0.9881 \\
$\kappa^{\prime}$ & 1.0027 \\
$\lambda_{1 u}$ & $-1.85 \times 10^{-5}$ \\
$\lambda_{1 d}$ & $3.70 \times 10^{-5}$ \\
$\lambda_{1 s}$ & 0.0 \\
\hline
\end{tabular}

Table 7.2: Electroweak coupling constants as they are expressed in the Standard Model obtained from Table 10.2 of [87]

netic corrections are expressed as a multiplicative factor to the cross section [86], thereby canceling in the asymmetry.

The parity violating asymmetry from elastic scattering of longitudinally polarized electrons from ${ }^{4} \mathrm{He}$, modified from Chapter 2 to include electroweak radiative corrections, is

$$
A_{L R}=\frac{G_{F} Q^{2}}{4 \pi \alpha \sqrt{2}}\left[4 \rho^{\prime} \kappa^{\prime} \sin ^{2} \theta_{W}+6\left(\lambda_{1 u}+\lambda_{1 d}\right)+\left[\rho^{\prime}+2\left(\lambda_{1 u}+\lambda_{1 d}+\lambda_{1 s}\right)\right] \frac{2 G_{E}^{s}}{G_{E}^{p \gamma}+G_{E}^{n \gamma}}\right]
$$

where the $\rho^{\prime}, \kappa^{\prime}$, and $\lambda$ coefficients are obtained from [87] and whose values are listed in Table 7.2.

The theoretical asymmetries for both hydrogen and ${ }^{4} \mathrm{He}$ are calculated using Equations 7.1 and 7.3 assuming no strange quarks $\left(G^{s}=0\right)$ for comparison to the measured asymmetries. These calculated asymmetries are labeled $A_{\mathrm{NS}}^{\mathrm{He}}$ and $A_{\mathrm{NS}}^{\mathrm{H}}$ and the kinematic factors for both calculations are listed in Table 7.3. The values of the needed electromagnetic form factors and their uncertainties were taken from a phenomenological fit to the world data at low $Q^{2}$ [30]. These values were evaluated at the ${ }^{4} \mathrm{He}$ and ${ }^{1} \mathrm{H} Q^{2}$ and are listed in Table 7.4. The error on $G_{E}^{n}$ was significantly reduced in 2005 with the result from the BLAST measurement [88]. 


\begin{tabular}{|c|c|c|}
\hline \multicolumn{3}{|c|}{ Kinematic factors } \\
\hline & ${ }^{4} \mathrm{He}$ & $\mathrm{H}$ \\
\hline$E_{\text {eff }}$ & $2.75 \mathrm{GeV}$ & $3.176 \mathrm{GeV}$ \\
$Q_{\text {eff }}^{2}$ & $0.0772 \mathrm{GeV}^{2}$ & $0.1089 \mathrm{GeV}^{2}$ \\
$\theta_{\text {eff }}$ & $6.0900^{\circ}$ & $6.0112^{\circ}$ \\
\hline
\end{tabular}

Table 7.3: Values of Effective Kinematic Factors used for ${ }^{4} \mathrm{He}$ and $\mathrm{H}$ calculations of $A_{\mathrm{NS}}(\mathrm{NS}=$ no strange quarks)

\begin{tabular}{|c|c|c|c|c|}
\hline Form & \multicolumn{2}{|c|}{${ }^{4} \mathrm{He}$} & \multicolumn{2}{c|}{$\mathrm{H}$} \\
Factor & Value & Error & Value & Error \\
\hline \hline$G_{E}^{p}$ & 0.798 & 0.007 & 0.734 & 0.007 \\
$G_{M}^{p}$ & $\mathrm{~N} / \mathrm{A}$ & $\mathrm{N} / \mathrm{A}$ & 2.086 & 0.031 \\
$G_{E}^{n}$ & 0.0280 & 0.004 & 0.037 & 0.004 \\
$G_{M}^{n}$ & $\mathrm{~N} / \mathrm{A}$ & $\mathrm{N} / \mathrm{A}$ & -1.403 & 0.021 \\
$G_{A}^{T=1}$ & $\mathrm{~N} / \mathrm{A}$ & $\mathrm{N} / \mathrm{A}$ & 0.517 & 0.020 \\
$G_{A}^{T=0}$ & $\mathrm{~N} / \mathrm{A}$ & $\mathrm{N} / \mathrm{A}$ & 0.137 & 0.040 \\
\hline
\end{tabular}

Table 7.4: Values of form factors evaluated at $Q_{\mathrm{eff}}^{2}$ used for $A_{\mathrm{NS}}^{\mathrm{He}}$ and $A_{\mathrm{NS}}^{\mathrm{H}}$ calculations

The axial form factors needed for hydrogen are also listed in Table 7.4 and are calculated assuming a dipole form $[89,90]$. The total contribution of all axial terms for the $2005 \mathrm{H}$ measurement (including all radiative corrections and the anapole moment [85]) is $-0037 \pm 0.018$ ppm, the error being dominated by uncertainties in the anapole moment. Any sensitivity to strange quarks in the axial form factors are neglected.

If $G_{E, M}^{s}$ are assumed to be zero, the standard model calculation predicts $A_{\mathrm{NS}}^{\mathrm{H}}=-1.66 \pm$ $0.05(\mathrm{FF})$ at the 2005 kinematics $\left(Q^{2}=0.109 \mathrm{GeV}^{2}\right)$. The larger part of the error on this asymmetry is due to the uncertainty of $G_{E}^{n}$.

For $A_{\mathrm{NS}}^{\mathrm{He}}$, it is independent of nuclear structure and determined purely by electroweak parameters if we assume a pure isoscalar $0^{+} \rightarrow 0^{+}$transition. $D$-state and isospin admixtures and meson exchange currents are negligible at the level of $\sim 3 \%$ experimental fractional accuracy [91]. The calculated values for $A_{\mathrm{NS}}$ and measured $A_{L R}$ 's for both ${ }^{4} \mathrm{He}$ and $\mathrm{H}$ are 


\begin{tabular}{|c|c|c|}
\hline & ${ }^{4} \mathrm{He}$ & $\mathrm{H}$ \\
& $Q^{2}=0.077 \mathrm{GeV}^{2}$ & $Q^{2}=0.109 \mathrm{GeV}^{2}$ \\
\hline$A_{L R}$ & $+6.40 \pm 0.23 \pm 0.12$ & $-1.60 \pm 0.12 \pm 0.05$ \\
\hline$A_{\mathrm{NS}}$ & +6.37 & $-1.66 \pm 0.05$ \\
\hline
\end{tabular}

Table 7.5: Calculated $A_{\mathrm{NS}}^{\mathrm{H}}, A_{\mathrm{NS}}^{\mathrm{He}}$ and measured $A_{L R}$ for $2005{ }^{4} \mathrm{He}$ and H Kinematics displayed for the 2005 kinematics in Table 7.5.

Comparing $A_{\mathrm{NS}}^{\mathrm{H}}$ to our measurement $A_{L R}$ in Table 7.5, we extract the following linear combination of strange form factors from the 2005 data of HAPPEX-H:

$$
G_{E}^{s}+0.088 G_{M}^{s}=0.007 \pm 0.011(\text { stat }) \pm 0.004(\text { syst }) \pm 0.005(\mathrm{FF})
$$

at $Q^{2}=0.109 \mathrm{GeV}^{2}$ [91]. The result for $G_{E}^{s}$ from the 2005 run of HAPPEX-He was

$$
G_{E}^{s}=-0.02 \pm 0.014 \text { (stat) } \pm 0.007 \text { (syst) }
$$

at $Q^{2}=0.077 \mathrm{GeV}^{2}[91]$ Combining these two results allows us to separate the electric and magnetic strange form factors (discussed in Section 7.3).

The 2004 HAPPEX results were $G_{E}^{s}+0.080 G_{M}^{s}=0.030 \pm 0.025 \pm 0.006 \pm 0.012(\mathrm{FF})$ at $Q^{2}=0.099 \mathrm{GeV}^{2}$ for hydrogen and $G_{E}^{s}=-0.038 \pm 0.042 \pm 0.010$ at $Q^{2}=0.091 \mathrm{GeV}^{2}$ for ${ }^{4} \mathrm{He}$ and are displayed in Figure 7.1 with $1 \sigma$ error bands along with three other $A_{L R}$ measurements $[92,93,94]$. The $1 \sigma$ error bands are a quadrature sum of the statistical and systematic errors.

The SAMPLE experiment [92] measured $G_{M}^{s}$ at $Q^{2}=0.1 \mathrm{GeV}^{2}$ with parity-violating electron-proton scattering at backward angles, a measurement primarily sensitive to a linear combination of $G_{M}^{s}$ and $G_{A}^{s}$. The calculation of $G_{A}^{s}$ from Zhu et al. [85] provided a constraint 
on $G_{M}^{s}$, and this is the "SAMPLE with $G_{A}$ calculation" band shown in Figures 7.1 and 7.2.

A4's measurement [93] of $A_{L R}$ uses electron-proton scattering at forward angles $\left(30^{\circ}<\right.$ $\theta<40^{\circ}$ ) and $Q^{2}=0.108 \mathrm{GeV}^{2}$ and is sensitive to the linear combination of strange form factors $G_{E}^{s}+0.106 G_{M}^{s}$.

The G0 band in Figure 7.1 is a measurement of $A_{L R}$ with electron-proton scattering at forward angles over a $Q^{2}$ range beginning at the low end with $0.122 \mathrm{GeV}^{2}$ [94]. At this $Q^{2}$, their sensitivity is to the linear combination $G_{E}^{s}+0.106 G_{M}^{s}$.

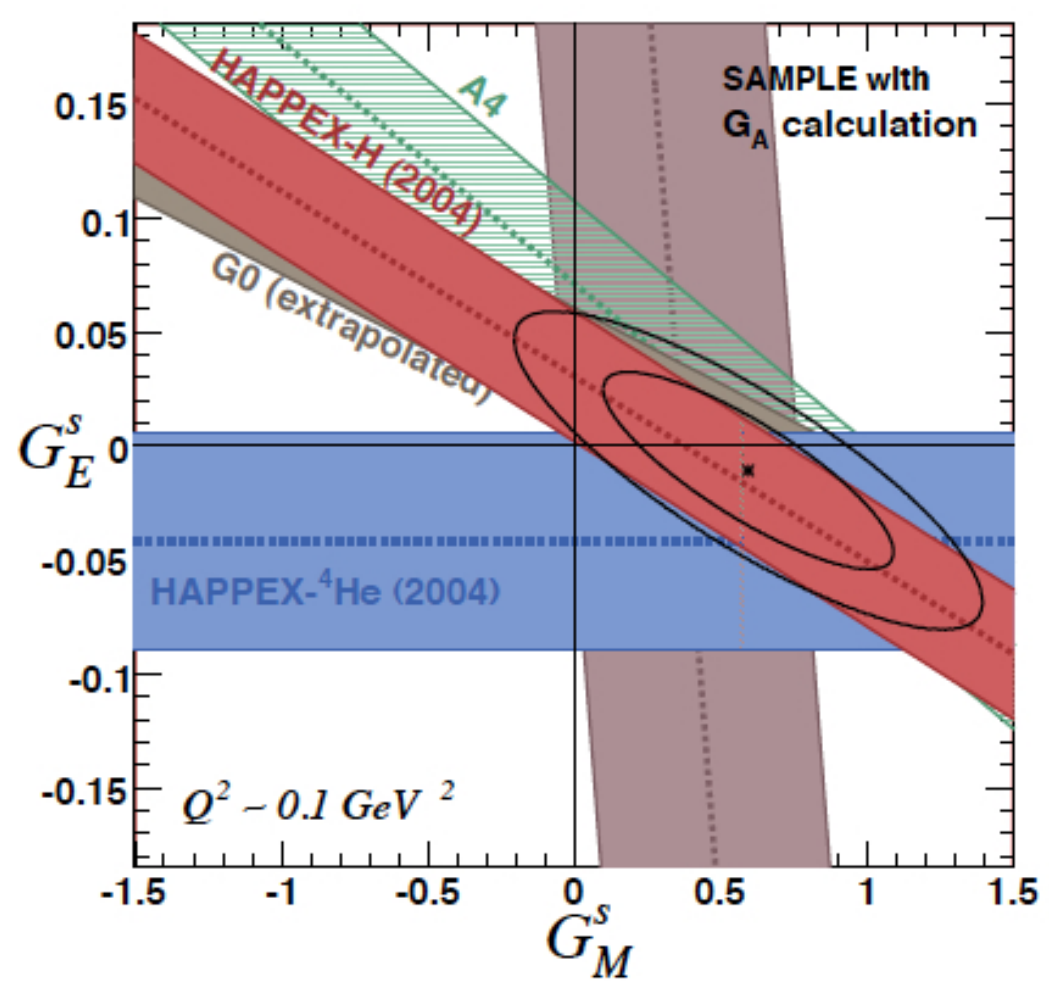

Figure 7.1: The five $A_{L R}$ measurements from $Q^{2}=0.09-0.11 \mathrm{GeV}^{2}$ with $1 \sigma$ error bands combining statistical and systematic uncertainties. Also displayed are the $68 \%$ and $95 \%$ C.L. ellipses from the results of the five measurements. Only 2004 HAPPEX results are included in the fit.

The dot in Figure 7.1 shows the best fit value of the five measurements, $G_{E}^{s}=-0.011 \pm$ 0.028 and $G_{M}^{s}=0.594 \pm 0.327$ (with a -0.83 correlation). The ellipses around the best fit 
are the $68 \%$ and $95 \%$ confidence levels for the combined result.

The 2005 HAPPEX results are plotted with the other three $A_{L R}$ measurements just discussed and are displayed in Figure 7.2. The best fit dot in the figure includes both the 2004 and 2005 HAPPEX measurements giving the results $G_{E}^{s}=-0.008 \pm 0.016$ and $G_{M}^{s}=0.324 \pm 0.215$ (with a -0.85 correlation). All measurements are at $Q^{2} \sim 0.1 \mathrm{GeV}^{2}$.

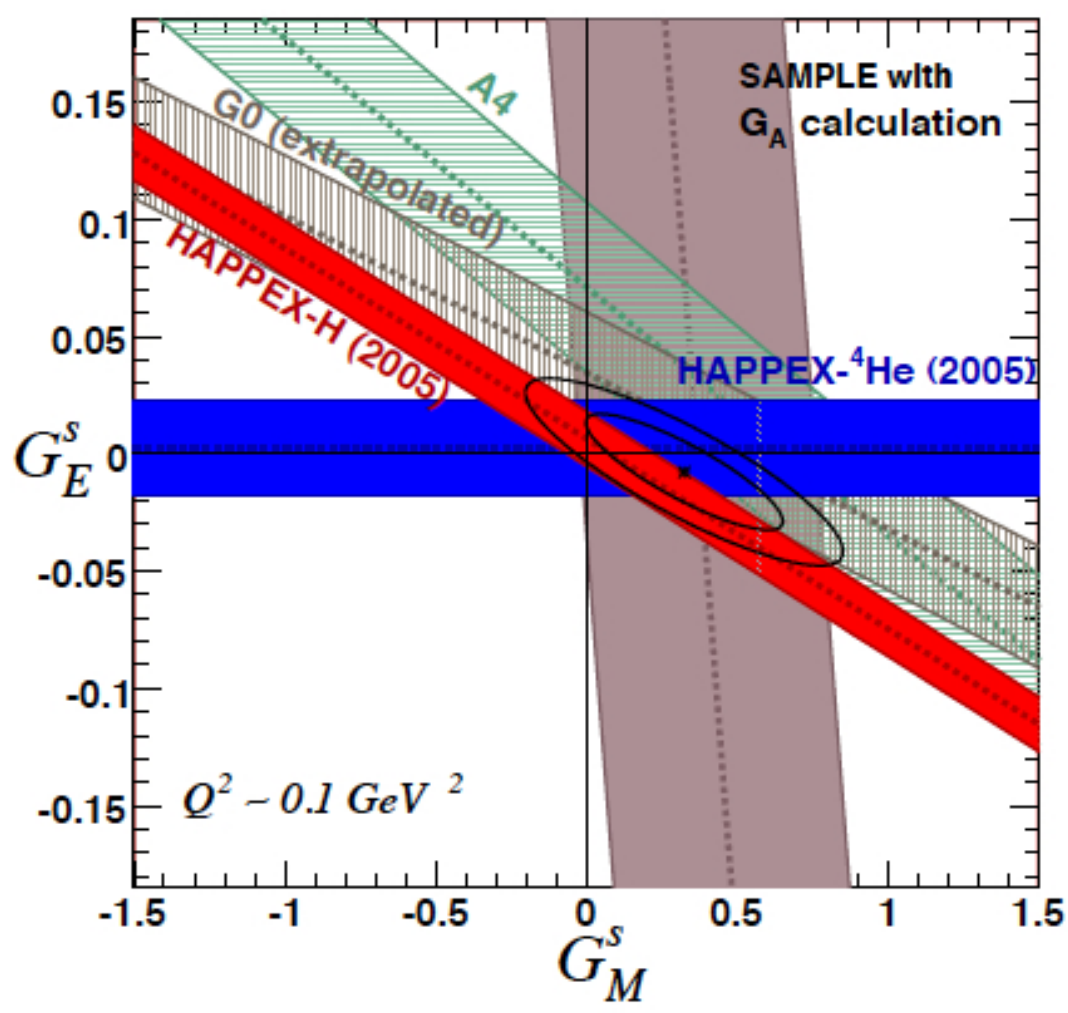

Figure 7.2: The five $A_{L R}$ measurements from $Q^{2}=0.09-0.11 \mathrm{GeV}^{2}$ with $1 \sigma$ error bands combining statistical and systematic uncertainties. Also displayed are the $68 \%$ and $95 \%$ C.L. ellipses from the results of the five measurements. The best fit dot in the figure include both the 2004 and 2005 HAPPEX results are included here.

The fit here is done by extrapolating all measurements to a common $Q^{2}=0.1 \mathrm{GeV}^{2}$. It was assumed that $G_{E}^{s} \propto Q^{2}$ and $G_{M}^{s}$ is constant which is consistent with the assumptions made in [95]. The assumed $Q^{2}$ dependence of $G_{E}^{s}$ is really of little consequence since a fit 
assuming both $G_{E}^{s}$ and $G_{M}^{s}$ constant gives similar results. Additionally, a more elaborate $Q^{2}$ evolution e.g. a Galster parameterization for $G_{E}^{s}$ and a dipole form for $G_{M}^{s}$ with the nucleon form factor "dipole mass", does not qualitatively alter the results of the fit at low $Q^{2}[95,49]$

\subsection{Interpretation and Conclusions}

The world data including only the 2004 HAPPEX results was consistent with a $5 \%$ to $10 \%$ contribution to $1 \sigma$ from strange quarks to the proton magnetic moment (Figure 7.1 ). The results including the 2005 data from HAPPEX (Figure 7.2) are consistent with zero and suggest that only a very small contribution of strange quarks to the proton magnetic moment and the charge distribution is possible.

The results from the 2004 and 2005 HAPPEX measurements alone are shown in Figure 7.3. From these, a best fit value of $G_{E}^{s}=-0.005 \pm 0.019$ and $G_{M}^{s}=0.18 \pm 0.27$ at $Q^{2}=0.1 \mathrm{GeV}^{2}$ (with a 0.87 correlation). The HAPPEX measurements alone give consistent and similarly precise results as the world data. The HAPPEX results are not altered by the addition of $[92,93,94]$ as the confidence interval ellipses cover roughly the same area with or without including the world data (compare Figures 7.2 and 7.3). The HAPPEX results allow for a clean determination of the strange vector form factors as they are rather insensitive to variations in $G_{A}^{s}$ due to uncertainties in its determination [49].

Theoretical models placing values on $G_{E}^{s}$ and $G_{M}^{s}$ at low $Q^{2}[96,97,98,99,23,24,25]$ are displayed in Figure 7.3. The results favor the predictions in [23, 24, 25].

There are measurements of parton distribution functions indicating a sizeable net $s \bar{s}$ 


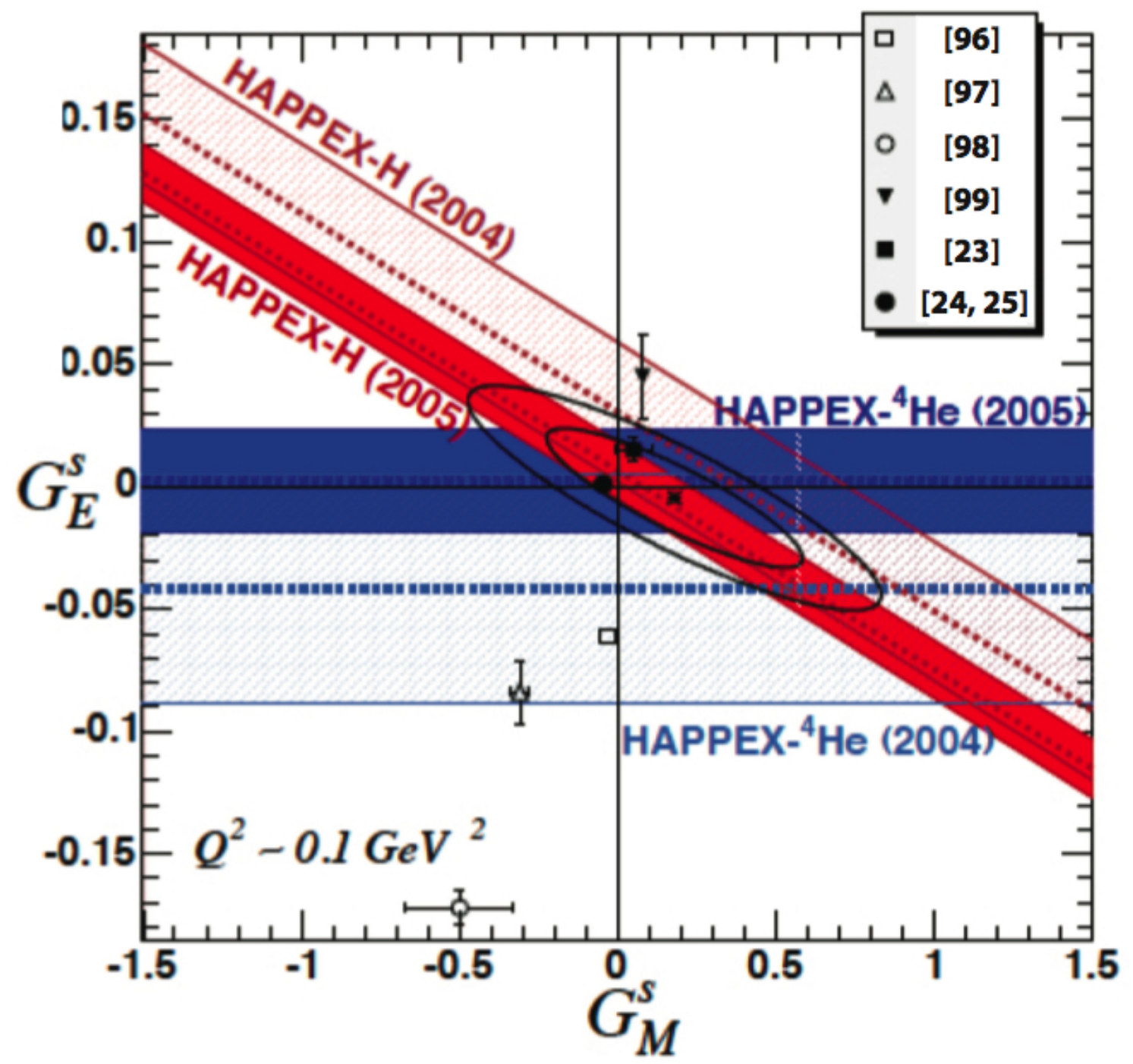

Figure 7.3: HAPPEX results from 2004 and 2005 measurements along with theoretical predictions for $G_{E}^{s}$ and $G_{M}^{s}$ plotted with their uncertainty estimates. The ellipses are the $68 \%$ and $95 \%$ confidence levels. 
content contributing to the proton momentum, and strange quark contributions to electric and magnetic properties of the proton are also predicted by several theoretical models [96, 97, 98]. Yet present experimental results are consistent with no strangeness dynamics, i.e. no $s \bar{s}$ separation in the nucleon.

Reported here are the most precise constraints on the strange form factors at $Q^{2} \sim 0.1$ $\mathrm{GeV}^{2}$. These results are consistent within errors of other parity-violating measurements, and leave little space for observable nucleon strangeness dynamics at low $Q^{2}$. Theoretical uncertainties, particularly with regard to the assumption of charge symmetry [100], prevent significant improvement to the measurements reported here.

Perhaps most impressive in these measurements was the unprecedented control over helicity-correlated asymmetries on the electron beam. Never before has the difference in position of an electron beam of opposite helicities been controlled on the level of atomic dimensions.

\subsection{Future Measurements}

Future Jefferson Lab experiments aim to measure parity-violating asymmetries an order of magnitude smaller with even greater precision. There are no future measurements planned to investigate the strangeness of the nucleon after the recent completion of HAPPEX-III (see below). Nuclear structure and physics beyond the Standard Model will all be investigated. Controlling systematics both experimentally and theoretically will, as is usual, be a certain challenge. 


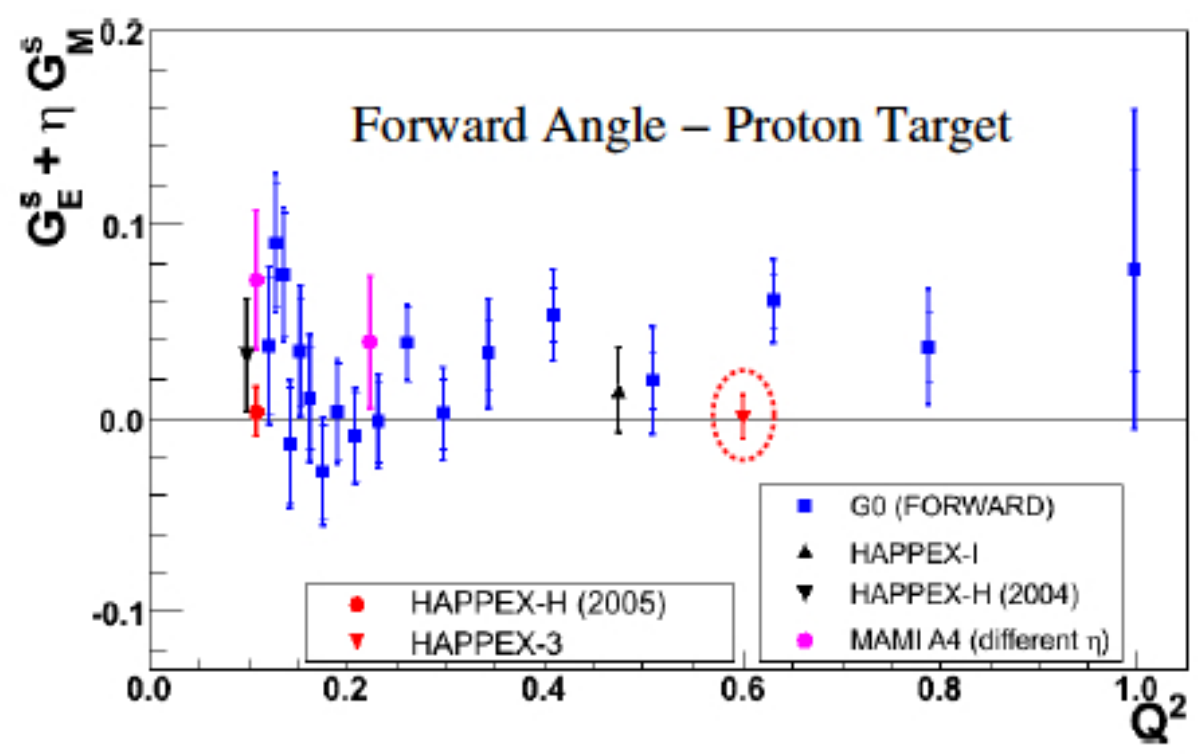

Figure 7.4: World data of $G_{E}^{s}+\eta G_{M}^{s}$ at forward angle on proton targets as a function of $Q^{2}$.

\subsubsection{Strange Vector Form Factors}

There were data from [94] consistent with non-zero strange quark contributions to nucleonic electric and magnetic properties at $Q^{2}>0.5 \mathrm{GeV}^{2}$, as the world data in Figure 7.4 of $G_{E}^{s}+\eta G_{M}^{s}$ from $0<Q^{2}<1 \mathrm{GeV}^{2}\left(\eta=\tau G_{M}^{p \gamma} / \epsilon G_{E}^{p \gamma}\right)$ shows. A HAPPEX experiment [101] just completed in Hall A measured $G^{s}+\eta G_{M}^{s}$ at $Q^{2}=0.6 G e V^{2}$ to definitively determine whether there is a significant strange quark contribution to the proton form factors at high $Q^{2}$. A backward angle measurement of $G_{M}^{s}$ at $Q^{2}=0.6 \mathrm{GeV}^{2}$ by the $\mathrm{G} 0$ collaboration will also help answer this question.

\subsubsection{Nuclear Structure}

PREX will use parity-violating elastic electron scattering from a ${ }^{208} \mathrm{~Pb}$ nucleus to measure the radius of the neutron skin, $R_{n}$, to a $1 \%$ precision. This measurement is an important 
parameter in calculations for neutron star structure, as well as for understanding possible exotic phases of dense matter, the proton fraction in matter rich with neutrons, and for measurements of the electroweak mixing angle in atomic physics [102].

\subsubsection{Physics Beyond the Standard Model}

$Q_{\text {weak }}$ will measure with elastic parity-violating electron-proton scattering the weak charge of the proton, $1-4 \sin ^{2} \theta_{W}$, at $Q^{2}=0.03 \mathrm{GeV}^{2}$ with $4 \%$ precision [103]. It hopes to constrain the running of $\sin ^{2} \theta_{W}$ to a $0.3 \%$ level and to $10 \sigma$ of the Standard Model. The measurement is sensitive to the $Z$-electron axial and $Z$-quark couplings, $C_{1 q}$, particularly the measurement of $2 C_{1 u}+C_{1 d}$. Previous measurements make precise determination of these couplings possible, which are sensitive to physics beyond the Standard Model including additional gauge bosons, supersymmetry, and leptoquarks.

Another upcoming parity-violating test of the Standard model will be a measurement of the weak charge of the electron $Q_{W}^{e}$ to a fractional accuracy of $2.3 \%$ at an average $Q^{2}$ of $0.0056 \mathrm{GeV}^{2}$. In the Standard Model, the $Q_{W}^{e}$ measurement yields a determination of the weak mixing angle $\sin ^{2} \theta_{W}$ with an uncertainty of \pm 0.00026 (stat) \pm 0.00013 (syst), an accuracy similar the most precise determinations from high energy colliders. Therefore, this result could potentially influence the central value of this fundamental electroweak parameter, and could be important in deciphering new physics signals at the Large Hadron Collider (LHC) [104].

A further measurement searching for physics beyond the Standard Model will be made using parity-violating deep inelastic scattering of polarized electrons (PVDIS) to high precision to search for new physics in lepton-quark current interactions. It will be sensitive to 
axial hadronic and weak neutral currents. The Standard Model predictions of weak current coupling constants have not been tested with a high degree of precision. Searching comprehensively for physics beyond the Standard Model requires precision measurements of all leptonic and semi-leptonic coupling constants as a complement to direct searches at the LHC [105]. To aim for a precision of $0.6 \%$ on the parity-violating asymmetry, a new solenoid spectrometer (SoLID) and a $11 \mathrm{GeV}$ beam will be used. There are also stringent requirements on beam polarimetry $(0.4 \%)$. The measurement will also search for higher twist effects in nucleon scattering at high $x$ and study charge symmetry violation in the nucleon. 


\section{Bibliography}

[1] E. Noether. Invariante Variationsprobleme. Nachr. v. d. Ges. d. Wiss. zu Göttingen, pages S. 235-257, 1918.

[2] T. D. Lee and C. N. Yang. Question of Parity Conservation in Weak Interactions. Phys. Rev., 104:254-258, 1956.

[3] C. S. Wu, E. Ambler, R. W. Hayward, D. D. Hoppes, and R. P. Hudson. Experimental Test of Parity Conservation in Beta Decay. Phys. Rev., 105:1413-1415, 1957.

[4] C. Y. Prescott et al., Further Measurements of Parity Nonconservation in Inelastic electron Scattering. Phys. Lett. B., 84:524-528, 1979.

[5] A. Pickering, Constructing Quarks, The University of Chicago Press, 1984.

[6] J. Ashman et al., Phys. Lett. B 206 (1988)

[7] D. B. Kaplan and A. Manohar, Nucl. Phys. B 310 (1988) 527.

[8] H. L. Lai et al., Phys. Rev. D 55 (1997) 1280.

[9] B. Borasoy and U.-G. Meissner, Ann. Phys., 254 (1997) 192, and references therein.

[10] K. Abe et al., Phys. Lett. B 405 (1997) 180.

[11] B. Adeva et al., Phys. Rev. D 58 (1998) 112002.

[12] G. Alterelli et al., Acta Phys. Polon. B 29 (1998) 1145.

[13] E. Leader et al., Phys. Lett. B 462 (1999) 189.

[14] H. Lipkin and M. Karliner, Phys. Lett. B 461 (1999) 280.

[15] D. H. Beck and B. R. Holstein, Nucleon Structure and Parity-Violating Electron Scattering. Int. J. Mod. Phys. E, E10:1, 2001.

[16] R. L. Jaffe, Phys. Lett. B 229 (1989) 275.

[17] H. W. Hammer, U. G. Meißner, and D. Dreschel, Phys. Lett. B367, 323 (1996).

[18] W. Koepf, E. M. Henley, S. J. Pollock, Phys. Lett. B 228 (1992) 11. 
[19] M. J. Musolf, M. Burkhardt, Z. Phys. C 61 (1994) 433.

[20] P. Geiger, N. Isgur, Phys. Rev. C 55 (1997) 299.

[21] E. J. Beise et al., Progress in Particle and Nuclear Physics 54 (2005) 289-350.

[22] S. J. Dong, K. F. Liu, A. G. Williams, Phys. Rev. D 58 (1998) 074504.

[23] R. Lewis, W. Wilcox, R. M. Woloshyn, Phys. Rev. D 67 (2003) 013003.

[24] D. B. Leinweber et al., Phys. Rev. Lett. 97:022001, 2006.

[25] D. B. Leinweber et al., Phys. Rev. Lett. 94:212001, 2005.

[26] M. J. Musolf et al., Intermediate-energy semileptonic probes of the hadronic neutral current., Phys. Rep., 239, 1994.

[27] M. N. Rosenbluth, Phys. Rev. 79, 615 (1950).

[28] C.E. Hyde-Wright and K. de Jager, Ann. Rev. Nucl. Sci. 54, 217 (2004).

[29] S. Galster et al., Nucl. Phys. B32, 221 (1971).

[30] J. Friedrich and T. Walcher, Eur. Phys. J. A. 17, 607 (2003).

[31] J. J. Kelly, Phys. Rev. C70 , 068202 (2004).

[32] J. D. Walecka, Electron Scattering for Nuclear and Nucleon Structure (Cambridge University Press, Cambridge, UK, 2001).

[33] J. D. Walecka, Theoretical Nuclear and Subnuclear Physics (World Scientific Publishing Co. Pte. Ltd., Singapore, 2004).

[34] G. A. Miller. Nucleon Charge Symmetry Breaking and Parity Violating Electron-Proton Scattering. Phys. Rev. C, 57:1492 1505, 1998.

[35] K. S. Kumar and P. A. Souder. Strange Quarks and Parity Violation. Prog. Part. Nucl. Phys., 45:S333-S395, 2000.

[36] M. J. Ramsey-Musolf, Phys. Rev. C 60 (1999) 015501.

[37] B. Mueller et al., Measurement of the proton's neutral weak magnetic form factor Physical Review Letters, 78:3824, 1997.

[38] D. T. Spayde et al., Parity violation in elastic electron-proton scattering and the proton's strange magnetic form factor. Physical Review Letters, 84:1106, 2000.

[39] H. Weigel et al., Phys. Lett., B353:353, 1995.

[40] S. Ramavataram, E. Hadjimichael, and T. W. Donnelly, Phys. Rev. C 50, 1175 (1994).

[41] M. J. Musolf and T. W. Donnelly, Phys. Lett. B 318, 263 (1993). 
[42] Proposal to Jefferson Lab PAC 16, Constraining the Nucleon Strangeness Radius in Parity Violating Electron Scattering, 1999.

[43] Proposal to Jefferson Lab PAC 18, Parity Violation from ${ }^{4}$ He at Low $Q^{2}$ : A Clean Measurement of $\rho_{s}, 2000$.

[44] M. J. Musolf, R. Schiavila, and T. W. Donnelly, Phys. Rev. C 50, 2173 (1994).

[45] K. A. Aniol et al., Phys. Rev. C69, 065501 (2004).

[46] Grady Wilson Miller IV, Ph.D. thesis, Princeton Univerisity, 2001, Parity Violation in Forward Angle Elastic Electron-Proton Scattering.

[47] T. Maruyama et al., Appl. Phys. Lett. 85, 2640 (2004).

[48] B. Moffit, Elastic Scattering of Longitudinally Polarized Electrons from ${ }^{4} \mathrm{He}$, Ph.D. Thesis, The College of William and Mary, 2007.

[49] L. Kaufman, Precision Measurement of the Proton Neutral Weak Form Factors at $Q^{2} \sim 0.1 \mathrm{GeV}^{2}$, Ph.D. Thesis, University of Massachusetts, 2007.

[50] R. A. Mair et al., Anisotropies in Strain and Quantum Efficiency of Strained GaAs Grown on GaAsP. Phys. Lett. A212, 231-236, 1996.

[51] M. Baylac and M. Poelker, (2004), http://www.jlab.org/accel/injgroup/docs/2004/superlattice.ppt-1.ppt.

[52] L. Glesener, Target Density Fluctuation Monitoring in the HAPPEX Helium Parity Violation Experiment, 2005, Internal Technical Document.

[53] J. Berthot, P. Vernin, Nucl. Phys. News 9 (1990) 12.

[54] D. Marchand, Ph.D. Thesis, University of Blaise Pascal, Clermont-Ferrand, 1997.

[55] X. Zheng, Ph.D. Thesis, Massachusetts Institute of Technology, 2003.

[56] K. Unser, IEEE Trans. Nucl. Sci. NS 28, 2344 (1981).

[57] W. Barry, J. Heefner, and J. Perry, Electronic systems for beam position monitors at CEBAF, October 1990, Technical Report CEBAF-PR-90-023.

[58] W. Barry, A general analysis of thin wire pickups for high frequency beam position monitors, October 1990, Technical Report CEBAF-PR-90-024.

[59] D. Margaziotis et. al., Racetrack Cells for HAPPEX.

[60] R. Michaels, Hall A Status Report - 2004, 41 (2005).

[61] J. Alcorn et al., Nucl. Instr. and Meth A 522, 294 (2004).

[62] P. Brindza et al., IEEE Trns. Appl. Supercond. 11, 1594 (2001). 
[63] J. J. LeRose and P. Brindza, Hall A Status Report - 2005, 9 (2006).

[64] W. bertozzi, et al., Nucl. Instrum. and Meth., 191, 957 (1977).

[65] N. Liyanage, Ph.D. thesis, Massachusetts Institute of Technology, 1999.

[66] A. Vacheret and D. Lhuillier, Detector for Parity Experiments in Hall A at Jefferson Laboratory, 2002, Internal Technical Document.

[67] L. J. Kaufman, D. Lhuillier, and R. Suleiman, HAPPEX Technical note, http://hallaweb.jlab.org/experiment/HAPPEX/docs/PMT_linearity2.ps.gz (2005).

[68] A. Vacheret and D. Lhuillier, HAPPEX Technical note, http://hallaweb.jlab.org/experimentHAPPEX/docs/electron_detector.ps.gz (2002), See also http://www-dapina.cea.fr/Sphn/Parity/Happex2/Det/index.php.

[69] J. P. Jorda et al., Nucl. Instr. and Meth. A 412, 1 (1998).

[70] D. Neyret et al., Nucl. Instr. and Meth A 443, 231 (2000).

[71] R.L. Goldstein, User's Guide for KD*P $\&$ Lithium Niobate Q-Switches $\&$ Modulators for Q-Switching, Choping, \& Pulse Extraction, http://fastpulse.com/pdf/eomgenl.pdf.

[72] A.E. Siegman, Lasers, University Science Books, 1986, 671-672.

[73] G. D. Cates, et al. Nuclear Instr. and Meth A 278 (1989), 293-317.

[74] T. B. Humensky, et al., Nuclear Instr. and Meth A 512 (2004) 261-298.

[75] E. Collett, Polarized Light, Marcel Dekker, Inc., New York 1993.

[76] A. E. Siegman, Lasers, University Science Books, 1986, 665.

[77] K. D. Paschke, proceedings of PAVI Conference, 2006.

[78] D. A. Edwards and M.J Syphers, An Introduction to the Physics of High Energy Accelerators (Wiley-Interscience, New York, 1993).

[79] R. Holmes et al., Pan: The Parity Analyzer, 2006, http://physics.syr.edu/rsholmes/happex/pan/html/index.html.

[80] R. Brun and F. Rademakers, Nucl. Instr. and Meth. A389, 81 (1997).

[81] J. Grames, Jefferson Lab Technical Note JLAB-TN-05-028 (2005).

[82] J. K. Tuli, Nuclear Wallet Cards, 7th ed. (2005), www.nndc.bnl.gov/wallet

[83] T. Holmstrom, R. Feuerbach, and R. Michaels, $Q^{2}$ for HAPPEX-2, 2005, Internal Technical Document.

[84] W.-M. Yao et. al., (Particle Data Group), J. Phys. G 33, 119 (2006). 
[85] S.-L. Zhu et. al., Phys. Rev. D62, 033008 (2000).

[86] L. C. Maximon and W. C. Parke, Phys. Rev. C61, 045502, (2000).

[87] S. Eidelman et. al., Phys. Lett. B592, 1 (2004).

[88] Vitaliy Ziskin, Ph.D. thesis, Measurement of the Electric Form Factor of the Neutron at Low Momentum Transfers Using a Vector Polarized Deuterium Gas Target at BLAST, Massachusetts Institute of Technology, 2005.

[89] Y. Goto et. al., Phys. Rev. D62, 034017 (2000).

[90] A. Bodek, H. Budd, and J. Arrington, AIP Conference Proceedings (USA) 698, 148 (2003).

[91] A. Acha et. al., Phys. Rev. Lett. 98, 032301 (2007).

[92] D. T. Spayde et al., Phys. Lett. B583, 79 (2004).

[93] F. E. Maas et. al., Phys. Rev. Lett. 94, 152001 (2005).

[94] D. S. Armstrong et al., Phys. Rev. Lett. 95, 092001 (2005).

[95] R. Young et al., Phys. Rev. Lett. 97, 102002 (2006).

[96] N. W. Park and H. Weigel, Nucl. Phys. A451, 453 (1992).

[97] H. W. Hammer, U. G. Meißner, and D. Dreschel, Phys. Lett. B367, 323 (1996).

[98] H. W. Hammer and M. J. Ramsey-Musolf, Phys. Rev. C60, 045204 (1999).

[99] A. Silva, H.-C. Kim, and K. Goeke, Phys. Rev. D65, 014016 (2001).

[100] B. Kubis and R. Lewis, Phys. Rev. C74, 015204 (2006).

[101] K. D. Paschke et al., (2005), Jefferson Lab E05-109 proposal, A Measurement of Nucleon Strangeness at High $Q^{2}$.

[102] R. Michaels et al., (2006), Jefferson Lab E06-002 proposal, A Clean Measurement of the Neutron Skin of ${ }^{208} \mathrm{~Pb}$ through Parity Violating Electron Scattering.

[103] R. Carlini et al., (2005), Jefferson Lab E05-008 proposal, The Qweak Experiment: A Search for Physics at the TeV Scale via a Measurement of the Proton's Weak Charge.

[104] An Ultra-precise Measurement of the Weak Mixing Angle using Møller Scattering, 2008. Proposal to JLAB PAC-35.

[105] Precision Measurement of Parity-violation in Deep Inelastic Scattering Over a Broad Kinematic Range, 2009, Proposal to JLAB-PAC 